CAHIER DE RECHERCHE \#2009E

WORKING PAPER \#2009E

DÉPARTEMENT DE SCIENCE ÉCONOMIQUE

DEPARTMENT OF ECONOMICS

FACULTÉ DES SCIENCES SOCIALES

FACULTY OF SOCIAL SCIENCES

UNIVERSITÉ D’OTTAWA

UNIVERSITY OF OTTAWA

\title{
An Economic Model of Health-vs-Wealth Prioritization During COVID-19: Optimal Lockdown, Network Centrality, and Segregation ${ }^{1}$
}

\author{
Roland Pongou ${ }^{2}$, Guy Tchuente ${ }^{3}$, and Jean-Baptiste Tondji ${ }^{4}$
}

December 2020

\footnotetext{
${ }^{1}$ Address correspondence to: rpongou@uottawa.ca or rpongou@hsph.harvard.edu (R. Pongou); g.tchuente@kent.ac.uk (G. Tchuente); and jeanbaptiste.tondji@utrgv.edu (J-B Tondji). We thank conference participants at the DSE Winter School 2020, and seminar participants at the Lab on Applied Rationality and Institutions (LARI) for their valuable and insightful comments and suggestions.

${ }^{2}$ Dept. of Economics, University of Ottawa; and Harvard T.H. Chan School of Public Health, Harvard University

${ }^{3}$ School of Economics, University of Kent

${ }^{4}$ Dept. of Economics and Finance, The University of Texas Rio Grande Valley
} 


\begin{abstract}
We address the problem of finding the optimal lockdown and reopening policy during a pandemic like COVID-19 for a social planner who prioritizes health over short-term wealth accumulation. Agents are connected through a fuzzy network of contacts. The planner's objective is to determine the policy that contains the spread of infection below a tolerable incidence level, which maximizes the present discounted value of real income, in that order of priority. We show theoretically that the planner's problem has a unique solution. The optimal policy depends both on the configuration of the contact network and the tolerated infection incidence. Using simulations, we apply these theoretical findings to: (i) quantify the tradeoff between the economical cost of the pandemic and the infection incidence allowed by the social planner and show how this tradeoff depends on network configuration; (ii) evaluate the correlation between different measures of network centrality and individual lockdown probability, and derive implications for the optimal design of surveys on social distancing behavior and network structure; and (iii) analyze how segregation induces differential health and economic dynamics in minority and majority populations, also illustrating the crucial role of patient zero in these dynamics.
\end{abstract}

Key words: COVID-19, health-vs-wealth prioritization, economic cost, fuzzy networks, network centrality, segregation, patient zero, optimally targeted lockdown policy.

JEL classification: E61, H12, I18, J15, D85 


\section{Introduction}

The rapid spread of the novel coronavirus disease (COVID-19) has already affected 76,624,363 individuals and claimed 1,690,238 lives globally (JHU CSSE, December 20, 2020, 1:27 PM). Governments around the world are implementing a variety of strategies to contain this pandemic: prescriptions of social distancing and hygiene measures, extensive production of personal protection equipment (PPE), expansion of testing and hospital capacities, use of face masks and contact tracing, and racing towards developing vaccines. In particular, the enforcement of social distancing policies has led to the cumulative lockdown of over half of the world population (Buchholz, 2020). While this approach for mitigating the contagion has shown some positive results, the associated economic costs are considerable. The Gross Domestic Product in both advanced and developing countries has decreased significantly as a result of the COVID-19 pandemic (see Figure 1 below), but the economy is projected to recover if this health situation is dealt with properly.

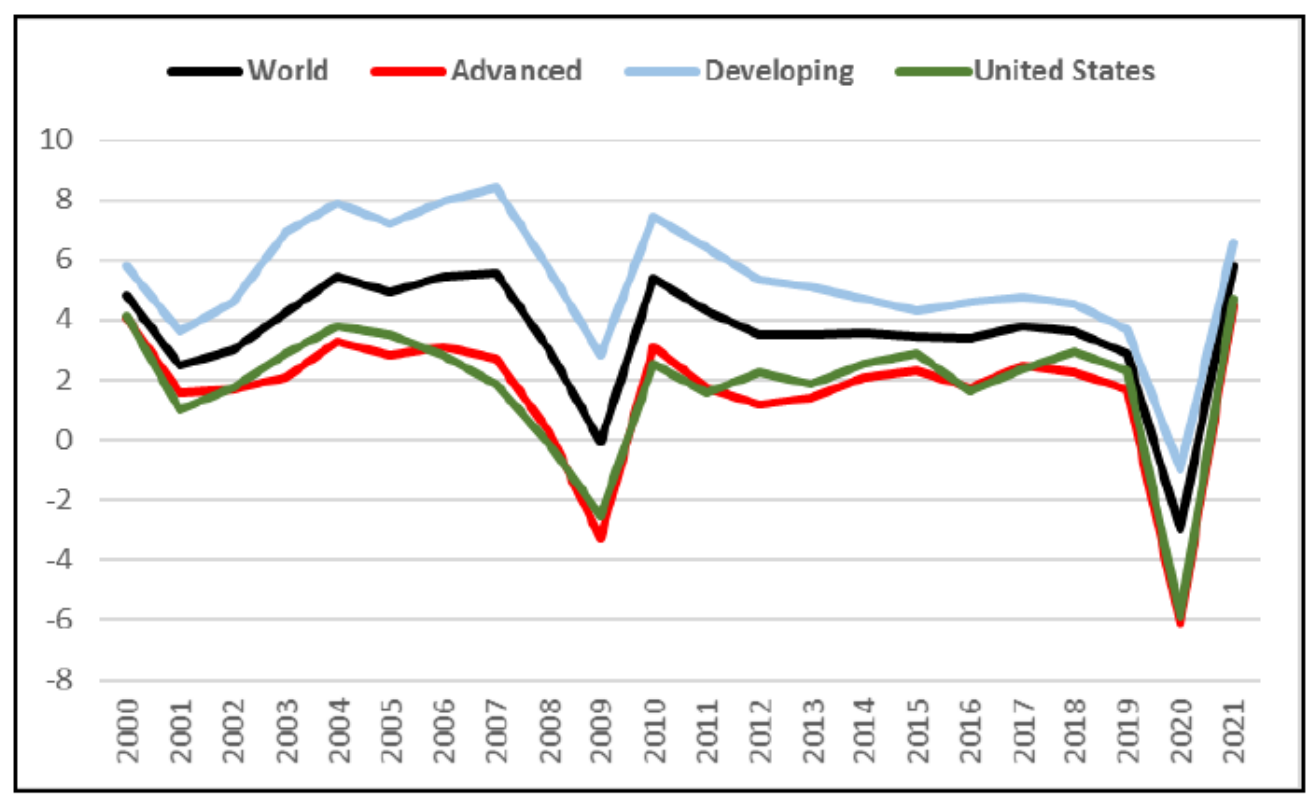

Source: World Economic Outlook, International Monetary Fund, April I4, 2020.

Figure 1: Gross Domestic Product, Percent Difference

In the United States (U.S), the Bureau of Labor Statistics reported on May 8, 2020 that total non-farm payroll employment fell by 20.5 million in April 2020, and the unemployment rate rose 
to 14.7 percent. In the United Kingdom, the National Institute for Economic and Social Research predicts that a prolonged lockdown could contract the economy by $15 \%$ to $25 \%$ in the second quarter of 2020 (Whyte, 2020). Additionally, only a small fraction of the jobs currently available in major economies are being done remotely. Dingel \& Neiman (2020) estimate that in the U.S, only $35 \%$ of jobs can be performed from home. Such a situation may not be economically viable in the long run. The important economic and social costs implied by quasi-complete lockdown is forcing governments and policymakers to think about less costly alternatives that might consist of imposing quarantine measures only on certain individuals while letting others go back to work. This new development raises the question of how to design optimally targeted lockdown policies, and how these policies are likely to affect different population subgroups.

In this paper, we address these important questions in a society that prioritizes health over short-term wealth accumulation, and where agents are connected through a fuzzy network of physical contacts. We develop a theoretical model, which we apply to analyze: (1) the effect of network structure on the dynamics of optimal lockdown, infection, recovery, death, and economic costs; (2) the tradeoff between public health and the economic cost of the pandemic; (3) how different measures of network centrality affect the probability of being sent to lockdown; and (4) how segregation induces differential health and economic dynamics for minority and majority populations following lockdowns.

We view our main question as a planning problem, and our assumption that health is prioritized over short-term economic gains is consistent with several recent observations. Indeed, a survey conducted in Canada, China, France, Germany, India, Japan, Mexico, Saudi Arabia, South Korea, the UK and the U.S shows that on average, $67 \%$ of the people interviewed highly prioritize saving as many lives as possible even if it costs the economy (John, 2020). In another study conducted in the U.S and UK, Heap et al. (2020) find that health is highly valued over wealth. Recently, when asked about relaxing travel restrictions for international travelers, Canadian Prime Minister Justin Trudeau cited public health over the economy as a top priority: "As we look at those next steps we need to make sure we are keeping Canadians safe, first and foremost." (Aiello, 2020, n.d). The latter is in line with a statement of Stiglitz (2020, n.d): "There can be no economic recovery until the virus is contained, so addressing the health emergency is the top priority for policymakers." As we argue later, our approach is more general and flexible, as it helps us avoid 
the difficult problem of giving a precise monetary value to health and life $!^{1}$

We now describe our model and state the social planner's objective problem:

A: Agents (including individuals and social infrastructures) are connected through a fuzzy network of physical contacts through which the virus is likely to spread. The fuzzy nature of the contact network implies that the intensity of interactions between agents is not necessarily binary, and it is likely to vary across relationships. ${ }^{2}$

B: At any point in time, an agent is one of the following five states: susceptible, infected, recovered, or dead. At time zero, all agents are susceptible. Susceptible agents can become infected, while infected agents can recover or die. Susceptible, infected and recovered agents can all be sent into lockdown. The dynamics of infection, recovery, and death follow a model that generalizes the classical SIR model (Kermack \& McKendrick, 1927, 1932) in two ways. First, whereas the classical model assumes a random matching technology, our model assumes any arbitrary network structure, and agents can only be infected through connections in the prevailing contact network if they have not been sent to lockdown. Second, whereas the classical model assumes three states (susceptible, infected, recovered), our model incorporates an additional state (death), and the lockdown variable. The lockdown variable is a key choice variable for the social planner, who uses it to modifies the structure of the prevailing social network in order to slow the spread of contagion.

C: The social planner's objective is to determine the lockdown policy that contains the spread of the infection below a tolerable incidence level, and that maximizes the present discounted value of real income (or alternatively, that minimizes the economic cost of the pandemic), in that order of priority. In other words, the social planner allocates the "work-from-home" rights to achieve these goals. An appeal of this lexicographic approach to the social planner's

${ }^{1}$ See Pindyck (2020) for the expression of a similar concern.

${ }^{2}$ Our model allows the interpretation of a contact network to be broad. At the micro-level, agents are individuals and the infrastructures (for example, public buildings, transportation infrastructures, shopping centers, parks, etc.) they interact with daily. At the macro-level, agents can be viewed as spatial entities such as countries or cities. Moreover, network configuration is arbitrary, making sense given that social structure varies a great deal across societies. For example, some societies are individualistic, while others are organized around extended family and ethnic networks (Deji, 2011, Pongou, 2010). 
problem is that it does not force us to assign a precise monetary value to health or to life. Rather, it allows some flexibility in how to design policies, with clear health and economic goals in mind. For instance, the social planner could set an incidence level that allows to keep the number of infected individuals below hospitals capacities, or she could set an incidence level that is high enough if she prioritizes short-term economic gains over health.

In order to solve the social planner's problem, we first characterize the dynamics of infection, recovery and death rates in our N-SIRD model with lockdown, and obtain a unique solution under classical conditions (Proposition 1). This unique solution shows that the rates of infection, recovery and death at any given time is a function of the lockdown variable and the initial network of contacts that captures social structure.

The planner's objective is achieved in two ordered steps. The first step consists of identifying the set of lockdown policies that contain the infection incidence (as dictated by the solution of the N-SIRD above) below a tolerable threshold, and the second step consists of choosing among those contagion-minimizing policies the policy which maximizes the discounted stream of economic surpluses. We show that this problem has a unique solution as well, and this solution depends on both the infection incidence level tolerated by the planner and the prevailing network of physical contacts that characterizes society (Proposition 2). This unique solution also shows that the tolerated infection incidence level and the prevailing network of physical contacts determine the dynamic of infection, recovery, death, and economic costs.

The same approach can be followed to design optimal exit policies from a situation of complete or quasi-complete lockdown. In this case, the social planner solves the inverse problem of the problem stated above, which consists of selecting which individuals should be released from lockdown first. It can be shown that the solution to this problem is unique, and that this solution has the same properties as the solution to the lockdown problem.

We develop several applications of our theoretical findings using simulations that rely on realistic data on transmission rates. First, we conduct a comparative statics analysis to study how the tolerated infection incidence level and the prevailing network of physical contacts determine the dynamic of infection, recovery, death, lockdown, and economic losses. We find that these two factors have a huge impact on these outcomes. For instance, we simulate two societies that are identical in every respect except in their social network structure. Agents in the first 
society are connected through a star network, whereas agents in the second society are connected through a small world network (Watts \& Strogatz, 1998). We find that the optimal lockdown policy differs significantly across the two societies, with around 20 percent and 35 percent being sent into lockdown (Figure 2), respectively. Similarly, the dynamics of infection differ, with the infection rate peaking earlier and being lower in the first society. However, the economic cost of the epidemic is overall higher in the small network world compared to the star network society.

Our analysis also allows us to appreciate how the infection incidence that is tolerated, by the social planner, affects the lockdown dynamic as well as the economic cost of the epidemic (Figure 4). We find that a higher tolerated incidence level results in lower lockdown rates and economic surplus loss. While this result illustrates the health-vs-wealth tradeoff faced by the social planner, it does not prescribe any resolution of this tradeoff, as this is ultimately based on the value that society puts on population health versus short-term economic gains.

Another application illustrates how different measures of network centrality affect the probability of lockdown. Simulations based on various network structures show that individuals that are more central in a network are more likely to be sent into lockdown. It does not imply that all agents have to be confined under the optimal policy, as the goal is simply to disconnect the prevailing contact network. The analysis therefore suggests that the recent quasi-universal lockdown implemented in certain countries around the world during the first wave of the COVID-19 pandemic was not necessarily optimal. While this approach was likely to disconnect the prevailing contact networks, it was more than necessary and resulted in untenable economic costs. Our analysis implies that only certain particular sectors of the society should be shut down, especially those sectors that attract large numbers of people, such as shopping centers, airports and other modes of public transportation, cities that play the role of economic or social hubs, schools, certain government buildings and other public services, entertainment fields, parks, beaches, among others. While this prediction was to be expected, what emerges from the analysis as less trivial is that some centrality measures are more predictive than others. In particular, we find that degree centrality correlates more highly to the probability of being sent into lockdown than betweenness or eigenvector or closeness centrality (see Table 1). This result has important empirical implications, especially in a context in which data on network structure are hard to collect. It indicates that information on the number of direct connections that an individual has in a network is more 
relevant for the design of lockdown policies than information on both direct and indirect connections (or information on the whole geometry of the network). As information on direct collections is also much easier and less costly to collect than information on network configuration, our analysis is likely to inform the optimal design of surveys that collect data on social distancing behavior and network structure during a pandemic like COVID-19.

Our final application studies the effect of segregation on the dynamics of infection, recovery, death, lockdown and economic losses among minority and majority populations. A striking feature of the COVID-19 pandemic is its highly unequal distribution among population subgroups within countries. In the U.S, for example, the black population, non-white Hispanics, and other communities of color have suffered a much greater burden of the pandemic (U.S. Centers For Disease Control and Prevention (2020) and McNeil (2020)), suggesting that both structural and individual factors might be driving its dynamics. To investigate this latter hypothesis, we simulate our data and use difference-in-differences analysis to determine if the dynamics of COVID-19 outcomes differ for minorities and majorities in the presence and in the absence of segregation. We find that these dynamics vary less across ethnic groups when the society is ethnically integrated. However, segregation induces significant minority-majority inequalities in outcomes, with the direction of these effects depending on the identity of patient zero (or the index patient, the first individual to be infected). If patient zero is a majority individual, segregation increases infections, deaths, lockdown rates and the economic costs of the pandemic for minorities relative to majorities. The analysis therefore indicates that segregation results in minorities bearing a greater burden of disease, death and economic losses during a pandemic like COVID-19 in the majority of scenarios regarding the starting point of the infection. More generally, our findings suggest that it is a combination of structural and individual factors that determine cross-group differences in outcomes 3

Our paper is related to several recent studies. In the economics literature on COVID-19, the canonical SIR model has been generalized in several directions to address a variety of problems. Recent generalizations include among others, Acemoglu et al. (2020) who propose a multi-risk SIR model, Bethune \& Korinek (2020) who study externalities of health interventions for infectious

\footnotetext{
${ }^{3} \mathrm{~A}$ similar argument has been made by Debnam Guzman et al. (2020) in a recent empirical study on the effects of identity and ethic divisions during COVID-19 pandemic in the United States.
} 
diseases in SIS and SIR models, Alvarez et al. (2020), Eichenbaum et al. (2020), and Prem et al. (2020) who analyze optimal non-pharmaceutical controls in SIR models, and Kuchler et al. (2020) and Harris (2020) who document the importance of social networks (e.g., Facebook) in the selection of targeted lockdown policies. While our model contains some of the ingredients of these other approaches, it differs in incorporating into the classical model two key elements, namely a lockdown variable and a network of contacts that is not necessarily random, and where agents are heterogeneous with respect to their positions and individual characteristics. Most importantly, we introduce a lexicographic approach to the planning problem, whereby the social planner's goal is to determine the lockdown policy that contains the spread of the infection below an acceptable incidence level, and that minimizes the economic cost of the pandemic, in that order of priority. An appeal of this approach is to help avoid the difficult problem of assigning a precise monetary value to health and life, which allows a great deal of flexibility and clarity in how to design optimal lockdown policies, with precise health and economic goals in mind. It also enables a transparent analysis of the tradeoff between public health and short-term economic prosperity.

Our model also complements and extends Peng et al. (2020) by allowing a diffusion dynamic similar to Lloyd et al. (2006). Additionally, since our network structure is not necessarily random, we are able to develop new applications. In particular, we show how different measures of network centrality affect the probability of lockdown, deriving new implications for the optimal design of surveys on social distancing and network structure. Also, our analysis shows how segregation induces differential health and economic dynamics among minority and majority populations, and highlights the key role of patient zero in these dynamics. Moreover, while our main application is to the current COVID-19 crisis, we believe that our model has implications for all other infections that spread through physical contacts.

The remainder of this paper is organized as follows. Section 2 presents the N-SIRD model with lockdown and a fuzzy network structure. Section 3 describes and solves the social planner's problem. In Section 4, we conduct simulation analyses to illustrate our theoretical findings. In Section 5, we discuss some policy implications and offer concluding remarks. Appendix A and Online Supplemental Materials contain theoretical derivations for the planning problem and additional simulation results. 


\section{N-SIRD Model with Lockdown}

In this section, we introduce a network SIRD model with lockdown. This model extends the classical SIR model (Kermack \& McKendrick, 1927, 1932) by incorporating a death state, a lockdown variable, and a non-random network of physical contacts through which infection is likely to spread. We shall first describe the classical SIR model followed by the exposition of our model.

\subsection{The Classical SIR Model}

The SIR model separates a population that has a constant size, $N$, into three disjoint health classes which change in continuous time $t$ :

$$
\begin{gathered}
S+I+R=N, \text { where }, \\
S=\text { Susceptible } \\
I=\text { Infected } \\
R=\text { Recovered. }
\end{gathered}
$$

We follow the convention in the epidemiological literature of dropping the time subscript of different states. We assume that an individual $i$ is in each of the three different states with the following probabilities:

$$
\begin{aligned}
& s_{i}=\operatorname{Prob} .(i \in S) \\
& x_{i}=\operatorname{Prob} .(i \in I) \\
& r_{i}=\operatorname{Prob} .(i \in R) .
\end{aligned}
$$

We denote by $\dot{s}, \dot{x}$, and $\dot{r}$ the derivative of these probabilities with respect to time. The SIR framework assumes random (uniform) mixing of infectious individuals with the rest of the population. Susceptible individuals contact other individuals in the population at a constant rate $\beta>0$. At any period, the probability that an individual $\mathrm{i}$ is infected, $x_{i}$, increases at the rate $\beta s_{i}$ due to new infections, while a fraction $\gamma$ of the infected individuals recover. Therefore, the evolution of variables $s_{i}, x_{i}$, and $r_{i}$ follows the following system of first-order ordinary differential equations 
(ODE):

$$
\begin{aligned}
\dot{s}_{i} & =-\beta s_{i} x_{i} \\
\dot{x}_{i} & =\beta s_{i} x_{i}-\gamma x_{i} \\
\dot{r}_{i} & =\gamma x_{i} .
\end{aligned}
$$

The parameter $\beta$ represents the expected amount of people an infected person infects per period. For instance, if we assume that the probability that an infected individual infects a susceptible person is $p$, and the average number of persons that a person is in contact with per period is $c$, then this individual will infect $p c$ persons per period, i.e., $\beta=p c$. Given that $1 / \gamma$ represents the number of days that an infected person has and can spread the virus, $\beta / \gamma$ is the total number of persons an infected person infects. This number is the basic reproduction number $R_{0}$ in epidemiology.

\subsection{The N-SIRD Model}

The N-SIRD model extends the classical SIR model to incorporate the death state (D) and a network of physical contacts through which an infection is likely to spread 4 In addition to the susceptible, infected, and recovered states in the SIR model, the classical SIRD framework also includes the death state, $D$, for individuals who died from the disease, so that:

$$
S+I+R+D=N
$$

We denote the probability that an individual $i$ dies from the infection as

$$
d_{i}=\operatorname{Prob} .(i \in D), \text { with } s_{i}+x_{i}+r_{i}+d_{i}=1
$$

Let $\kappa>0$ be the death probability, and $\dot{d}$ the infinitesimal change in death probabilities over time. The evolution of variables $s_{i}, x_{i}, r_{i}$, and $d_{i}$ in the classical SIRD model follows the following system

\footnotetext{
${ }^{4}$ Hethcote (2000) and a recent textbook by Brauer et al. (2012) present an overview of the class of SIRD models and some of their theoretical features in epidemiology. Anastassopoulou et al. (2020) and Fernández-Villaverde \& Jones (2020) apply these models to analyze the possible outcomes of the current COVID-19 pandemic.
} 
of equations:

$$
\begin{aligned}
\dot{s}_{i} & =-\beta s_{i} x_{i} \\
\dot{x}_{i} & =\beta s_{i} x_{i}-\gamma x_{i}-\kappa x_{i} \\
\dot{r}_{i} & =\gamma x_{i} \\
\dot{d}_{i} & =\kappa x_{i} .
\end{aligned}
$$

Like the SIR model, the SIRD model assumes random (uniform) mixing of infectious individuals with the rest of the population, with susceptible individuals contacting other individuals in the population at a constant rate $\beta>0$. We modify this assumption by assuming physical contacts take place through a non-directed and non-random fuzzy network, $A$. This network is represented by the adjacency matrix $\left(A_{i, j}\right)$, where $A_{i j} \in[0,1]$ represents the intensity at which individuals $i$ and $j$ are connected in the network $A$. The intensity of the relationship between two individuals could be interpreted as the degree or frequency of interactions between these individuals. Our model therefore generalizes the binary model in which:

$$
A_{i j}= \begin{cases}1 & \text { if } i \text { and } j \text { are connected in } A \\ 0 & \text { if }(i \text { and } j \text { are not connected in } A) \text { or }(i=j)\end{cases}
$$

Our model accounts for weak and strong ties. An individual $i$ can only interact with his direct neighbors in $A$. In the presence of a contact network $A$, equations (4)-(7) therefore change to the following system of first-order ODE:

$$
\begin{aligned}
\dot{s}_{i} & =-\beta s_{i} \sum_{j \in N}\left(A_{i j} x_{j}\right) \\
\dot{x}_{i} & =\beta s_{i} \sum_{j \in N}\left(A_{i j} x_{j}\right)-\gamma x_{i}-\kappa x_{i} \\
\dot{r}_{i} & =\gamma x_{i} \\
\dot{d}_{i} & =\kappa x_{i} .
\end{aligned}
$$

Equations (8)-(11) show the spread over time of an epidemic in a fixed population of individuals through a social structure $A$. Remark that the N-SIRD model generalizes both the SIR and SIRD models. In fact, if for each $i, j \in N, A_{i j}=1$, and $\kappa=0$, the N-SIRD corresponds to the random and uniform mixing transmission infection in the canonical SIR model. If for each $i, j \in N, A_{i j}=1$, and $\kappa>0$, the N-SIRD models equals the SIRD model. 


\subsection{The N-SIRD Model with Lockdown}

We now incorporate a lockdown variable into the N-SIRD model. This captures the fact that a social planner might decide to reduce the spread of the infection by enforcing a lockdown policy that modifies the structure of the existing social network. Denoting by $l_{i}=$ Prob. $(i \in L)$ the probability that a random individual $i$ is sent into lockdown. An individual $i$ for which $l_{i}=1$ is said to be sent into complete lockdown. Let us denote by $i$ the infinitesimal change in lockdown probabilities over time. The infection propagation in the N-SIRD model with the lockdown variable $l$ is governed by the following nonlinear differential equation:

$$
\begin{aligned}
\dot{s}_{i} & =-\beta\left[1-x_{i}-r_{i}-d_{i}\right]\left(1-l_{i}\right) \sum_{j \neq i}\left[A_{i j}\left(1-l_{j}\right) x_{j}\right] \\
\dot{x}_{i} & =\beta\left[1-x_{i}-r_{i}-d_{i}\right]\left(1-l_{i}\right) \sum_{j \neq i}\left[A_{i j}\left(1-l_{j}\right) x_{j}\right]-(\gamma+\kappa) x_{i} \\
\dot{r}_{i} & =\gamma x_{i} \\
\dot{d}_{i} & =\kappa x_{i}
\end{aligned}
$$

with the initial value point $\left(s_{i}(0), x_{i}(0), r_{i}(0), d_{i}(0)\right)$ such that

$$
s_{i}(0) \geq 0, x_{i}(0) \geq 0, r_{i}(0) \geq 0, d_{i}(0) \geq 0, \text { and } s_{i}(0)+x_{i}(0)+r_{i}(0)+d_{i}(0)=1 .
$$

Remark that in our model, any individual can be sent into lockdown regardless of whether the individual is susceptible, infected or recovered. Following the first two equations (12) and (13) of the system, a susceptible individual $i$ becomes infected only if: (a) she is not sent into complete lockdown $\left(l_{i} \neq 1\right)$; and (b) she is not connected to an infected individual $j$ who is not sent into complete lockdown $\left(A_{i j}>0, x_{j}>0\right.$, and $\left.l_{j} \neq 1\right)$. Also, remark that the lockdown variable only modifies the structure of the existing network in our model. It is not a state variable that excludes being in other states.

The proposition below states that the non-linear system of ODE (12)-(16) has a unique solution.

Proposition 1. The system of equations (12)-(16) has a unique solution $\mathcal{S}^{*}=\mathcal{S}^{*}(l, A, \beta, \gamma, \kappa)$. Proof. Equations (12)-(16) describe a general first-order general ordinary differential equations (ODE). Let $i \in N$ and $X_{i}=\left(s_{i}, x_{i}, r_{i}, d_{i}\right)^{T}$, where $T$ means "transpose". There exists a continuously differentiable function $\mathbb{F}_{i}$, such that $\dot{X}_{i}=\mathbb{F}_{i}\left(t, X_{i}\right)$. Consequently, the ODE admits 
a unique solution, $\mathcal{S}^{*}\left(l, A, \beta, \gamma, \kappa, X_{0}\right)$, thanks to the theorem of existence and uniqueness of a solution for first-order general ODE, where $X_{i}(0)=\left(s_{i}(0), x_{i}(0), r_{i}(0), d_{i}(0)\right)$ is the initial value point, and $l=\left(l_{i}\right)_{i \in N} \in[0,1]^{n}$ a lockdown policy.

\section{The Planning Problem: Optimal Lockdown}

The unique solution of the non-linear system of ODE (12)-(16) in section 2.3 depends on both the social network $A$ and the lockdown variable $l$. The planning problem consists of choosing $l$ optimally. A first approach to containing the spread of COVID-19 in many countries was to enforce a quasi-complete lockdown policy. While this approach has proved to slow the spread of the virus, its economic costs have been significant. Governments around the world have been implementing less costly alternatives consisting of sending only certain individuals into lockdown while letting others go back to work. This raises the question of whether an optimal lockdown policy exists, and, if it does, whether it is unique.

In this section, we answer this question for a social planner that prioritizes health over shortterm wealth accumulation, and we show that, under minimal conditions, there exists a unique optimally targeted lockdown policy. More formally, we assume that the social planner's problem consists of choosing the lockdown policy $l$ that:

1. contains the infection incidence level (or the relative number of new infections) below a tolerable threshold $\lambda$; and

2. minimizes the economic costs of the infection to the entire society, in this order of priority.

This lexicographic objective problem is formalized below.

Containing the spread of infection. Using (13), the first objective of the planner is to select a lockdown policy $l$ such that:

$$
\dot{x}_{i} \equiv \dot{x}_{i}(l) \leq \lambda \text {, where } \lambda \text { is a non-negative parameter. }
$$

Note that the system $(12,17)$ admits at least one solution. In fact, consider the policy $l$ where each individual is sent into complete lockdown, i.e, $l_{i}(t)=1$ for all $i \in N$ and $t$. Then, 
$\dot{x}_{i}=-(\gamma+\kappa) x_{i}$. Therefore, given any $\lambda \geq 0$, it follows that $\dot{x}_{i}(l) \leq \lambda$. In practice, the upper bound of the parameter $\lambda$ could be equal to the reproduction rate $R_{0}=\beta /(\gamma+\kappa)$ for a planner who seeks to eventually eliminate the disease.

Minimizing the economic costs of lockdown. The planner's second-order objective is to minimize the economic costs of lockdown by choosing from the set of policies that satisfy the first objective the policy that maximizes the present discounted value of real income. At any given period $t$, each individual $i$ possesses a capital level $k_{i}$, and a labor supply $h_{i}$. We assume, as in most SIR models, that individuals who recover from the infection are immune to the virus and must be released to the workforce. It follows that individuals in states $S, I$, and $R$ are the only potential workers in the economy. The individual labor supply depends on individuals' health states and probability of being into the lockdown:

$$
h_{i}=h\left(l_{i}, x_{i}, d_{i}\right) \text {, with } h \text { assumed to be continuous and differentiable in } x_{i}, l_{i} \text {, and } d_{i} \text {. }
$$

We assume that individual economic productivity decreases with illness, death, and being in lockdown:

$$
\frac{\partial h_{i}}{\partial l_{i}} \leq 0, \frac{\partial h_{i}}{\partial x_{i}} \leq 0, \text { and } \frac{\partial h_{i}}{\partial d_{i}} \leq 0
$$

Naturally, an individual who is working despite being infected and sick produces less compared to when this individual is healthy. Without loss of generality, we assume that capital is constant over time $\left(k_{i}(t)=k_{i}\right.$, for each $\left.t\right)$, and labor is the only variable input in the production. A combination of capital and labor supply generates output $y$ according to the following production function:

$$
y_{i}=Y_{i}\left(k_{i}, h_{i}\right)=Y_{i}\left(k_{i}, l_{i}, x_{i}, d_{i}\right)
$$

We assume that $Y_{i}$ is continuous and differentiable in each of its input variables. Moreover we make the following natural assumptions:

$$
\frac{\partial Y_{i}}{\partial k_{i}}>0, \frac{\partial Y_{i}}{\partial l_{i}} \leq 0, \frac{\partial Y_{i}}{\partial x_{i}} \leq 0, \frac{\partial Y_{i}}{\partial d_{i}} \leq 0, \text { and } \frac{\partial Y_{i}^{2}}{\partial^{2} v} \leq 0, \text { for } v \in\left\{k_{i}, l_{i}, x_{i}, d_{i}\right\}
$$


Other important variables of the problem include:

$$
\begin{aligned}
w_{i} & =\text { the individual cost of one unit of labor } \\
p & =\text { the price per unit of output } \\
\delta & =\text { the social planner's discount rate }
\end{aligned}
$$

The planner chooses $l \in[0,1]^{n}$ to maximize the aggregate surplus:

$$
\begin{aligned}
W(k, l, x, d): & =\sum_{i \in N} W_{i}\left(k_{i}, l_{i}, x_{i}, d_{i}\right) \\
& =\sum_{i \in N}\left\{\int_{0}^{\infty} e^{-\delta t}\left(p Y_{i}\left(k_{i}, l_{i}, x_{i}, d_{i}\right)-w_{i} h\left(l_{i}, x_{i}, d_{i}\right)\right) d t\right\}
\end{aligned}
$$

The social planner's problem. Finally, using optimal control theory, we can formalize the social planner's problem as described below:

$$
\begin{array}{cl}
\underset{\left(l_{i}\right)}{\operatorname{Maximize}} & \int_{0}^{\infty} e^{-\delta t} \sum_{i \in N}\left\{p Y_{i}\left(k_{i}, l_{i}, x_{i}, d_{i}\right)-w_{i} h\left(l_{i}, x_{i}, d_{i}\right)\right\} d t \\
\text { subject to } & \dot{x}_{i}=\beta\left[1-x_{i}-r_{i}-d_{i}\right]\left(1-l_{i}\right) \sum_{j \neq i}\left[A_{i j}\left(1-l_{j}\right) x_{j}\right]-(\gamma+\kappa) x_{i} \\
& \dot{x}_{i} \leq \lambda, \lambda \geq 0 \\
& \dot{r}_{i}=\gamma x_{i} \\
& \dot{d}_{i}=\kappa x_{i} \\
& \dot{s}_{i}=-\beta\left[1-x_{i}-r_{i}-d_{i}\right]\left(1-l_{i}\right) \sum_{j \neq i}\left[A_{i j}\left(1-l_{j}\right) x_{j}\right] \\
& X_{i}(0)=\left(s_{i}(0), x_{i}(0), r_{i}(0), d_{i}(0)\right)^{T} \text { given, with } \\
& s_{i}(0)+x_{i}(0)+r_{i}(0)+d_{i}(0)=1 .
\end{array}
$$

We have the following result.

Proposition 2. The social planner's problem (23) has a unique solution.

Proof. We denote $f_{i}=\beta\left[1-x_{i}-r_{i}-d_{i}\right]\left(1-l_{i}\right) \sum_{j \neq i}\left[A_{i j}\left(1-l_{j}\right) x_{j}\right]-(\gamma+\kappa) x_{i}$, and $W_{i}^{p}=$ $p Y_{i}\left(k_{i}, l_{i}, x_{i}, d_{i}\right)-w_{i} h\left(l_{i}, x_{i}, d_{i}\right)$. The function $l_{i}: t \longrightarrow l_{i}(t) \in[0,1]$ is continuous. The function $W_{i}^{p}$, and the objective function in (23) are continuous and differentiable. Moreover, $f_{i}$ and the right-hand sides of the laws of motion in (23) are all continuous and differentiable. It follows that 
the problem (23) admits a unique optimal path $\left\{l^{*}(t)\right\}$ of the control variable (and the states $\left\{x^{*}(t), r^{*}(t), d^{*}(t), s^{*}(t)\right\}$, given the initial conditions $X_{0}$ and the law of motion).

Proposition 2 states the existence and uniqueness of a solution to the social planner's problem. However, determining a closed-form solution is intractable. To gain some insight into the optimal dynamic of the lockdown policy and the resulting dynamics of infection, recovery and death rates and cumulative economic loss, and how these dynamics depend both on the topology of the contact network and the infection incidence level that the society is willing to tolerate, we resort to simulations in the next section 5

\section{A Simulation Analysis}

The parameters of the model are chosen to match the dynamic of the infection and recent papers on COVID-19. Following Alvarez et al. (2020), we use data from the World Health Organization (WHO) made public through the Johns Hopkins University Center for Systems Science and Engineering (JHU CCSE). The parameter $\beta$, the probability that an infected individual infects another individual to whom he or she is connected is assumed to be 0.2. The lifetime duration of the virus is assumed to be 18 days (see Acemoglu et al. (2020) and the references therein). The proportion of recovered closed cases is around 70\% for the USA, $93 \%$ for Germany, and $86 \%$ for Spain (JHU CCSE access on the 5 May 2020). We thus assume that the parameter governing the recovery of an infected patient is given by $\gamma=0.8 / 18$, and the parameter governing the death dynamic is given by $\kappa=0.2 / 18$. For simplification, we consider in the simulation the following functional form for the labour function $(h)$ and the production function $(Y)$ :

$$
\begin{aligned}
h_{i}\left(l_{i}, x_{i}, d_{i}\right) & =\left(1-\left(1-\phi_{i}\right) x_{i}\right)\left(1-l_{i}\right)\left(1-d_{i}\right), \phi_{i} \in[0,1] \\
Y_{i}\left(k_{i}, l_{i}, x_{i}, d_{i}\right) & =k_{i}^{\alpha}\left\{\left(1-(1-\phi) x_{i}\right)\left(1-l_{i}\right)\left(1-d_{i}\right)\right\}^{1-\alpha},
\end{aligned}
$$

\footnotetext{
${ }^{5}$ In the appendix, we extend the analysis of problem (23) that proves useful in showing how we obtain our simulated results.
} 
where $\phi_{i}$ represents the rate of change in productivity when individual $i$ is infected. Using (24):

$$
\begin{aligned}
\frac{\partial h}{\partial l_{i}} & =-\left(1-\left(1-\phi_{i}\right) x_{i}\right)\left(1-d_{i}\right) \leq 0 \\
\frac{\partial h}{\partial x_{i}} & =-\left(1-\phi_{i}\right)\left(1-l_{k}\right)\left(1-d_{i}\right) \leq 0 \\
\frac{\partial h}{\partial d_{i}} & =-\left(1-\left(1-\phi_{i}\right) x_{i}\right)\left(1-l_{i}\right) \leq 0
\end{aligned}
$$

With (25), and each of the equations (26), (27), and (28), it is straightforward to show that the conditions (19) and (21) on $h$ and $Y$, respectively, are satisfied.

In all the simulations, we consider $\phi_{i}=1$ and we have a stationary working population such that $h_{i}\left(l_{i}, x_{i}, d_{i}\right)=\left(1-l_{i}\right)$. As for the surplus function, we assume that $\alpha=1 / 3, p=1.2$, $w=p / 3$ and the level of capital is the same for all individual at all time period and normalized to $k_{i}=1$. The annual interest rate is assumed to be equal to $5 \% .6$

We represent the simulation results in a two-dimensional graphic, with days (or periods) in the horizontal axis, and the percentage of population affected for the variable (infection, lockdown, or death) illustrated on the vertical axis. In each period, a point in the graphic represents the average value of individual probabilities. For the economic cost, the vertical axis represents the percentage of economic loss relative to the economy without the pandemic.

In the analyses that follow, we illustrate: (a) how network configuration affects the dynamics of lockdown, health and economic costs (section 4.1); (b) the tradeoff between the tolerated infection incidence level and the economy (section 4.2); (c) how network centrality affects individual lockdown probability (section 4.3); and (d) how segregation and the identity of the patient zero (the first individual to be infected) induce differential outcomes among minority and majority populations in a society (section 4.4).

\subsection{Illustration I: The Role of Network Configuration}

In this first illustration of our theoretical analysis, we show how the structure of the contact network affects the optimal lockdown policy and the resulting dynamic of infection and death

\footnotetext{
${ }^{6}$ The values of the parameters of the production function are chosen for illustration purposes. Thus, quantitative outcomes of the model should be interpreted with caution.
} 
rates and economic cost, for a given infection incidence level. 7 For the sake of concreteness, we contrast two popular forms of networks, namely a star network (Graph (a) in Figure 2) and a small-world network (Graph (b) in Figure 2). These networks can be viewed as representing two societies of 10 individuals each, that are identical in all ways except the configuration of their contact network.

Figure 2: Simple Networks $(n=10)$

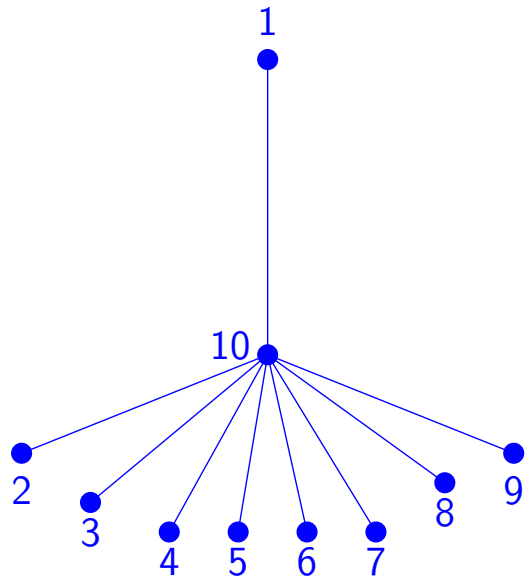

(a) Star Network

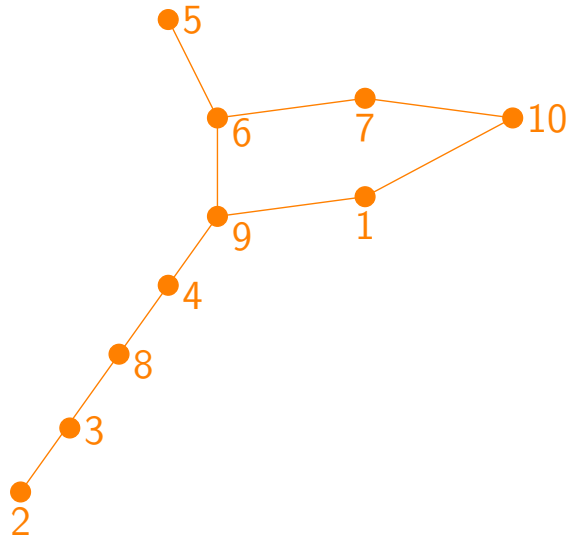

(b) Small World Network

In simulation, we assume that the incidence level is $\lambda=0.01$. The simulation results lead to the respective lockdown policies represented in Figure 3 a for these two societies. The dynamic of lockdown is significantly different for each society. The cumulative proportion of the population sent into lockdown peaks and flattens much earlier in the star-network society than in the smallworld-network society. While this cumulative proportion is a bit larger in the former society in the early period of the pandemic and plateaus at around 20 percent, it plateaus at 32 percent in the latter society. Similarly, the dynamic of the infection rate is very different across the two societies (Figure 3b), with the star-network society ultimately having a much lower infection rate. The lower lockdown and infection rates in the star-network society are simply explained by the fact that optimal lockdown simply requires isolating the hub, and once the hub is isolated, the infection can no longer spread from one individual to another individual. The situation is different

\footnotetext{
${ }^{7}$ This analysis is consistent with other studies showing that network configuration plays an essential role in the level of infection spread and information diffusion (Pongou \& Serrano, 2016, 2013).
} 
in the small-world network society, where several individuals have to be isolated to contain the infection below a chosen incidence level.

Similar to the dynamics of lockdown and infection, the dynamics of the death rate (Figure 3d) and the lost economic surplus (Figure 3c) are different across the two societies. Once again, the cumulative death rate and lost economic surplus due to the pandemic are ultimately lower in the star-network society.

Figure 3: Optimal Infection Dynamic in Simple Networks $(n=10)$

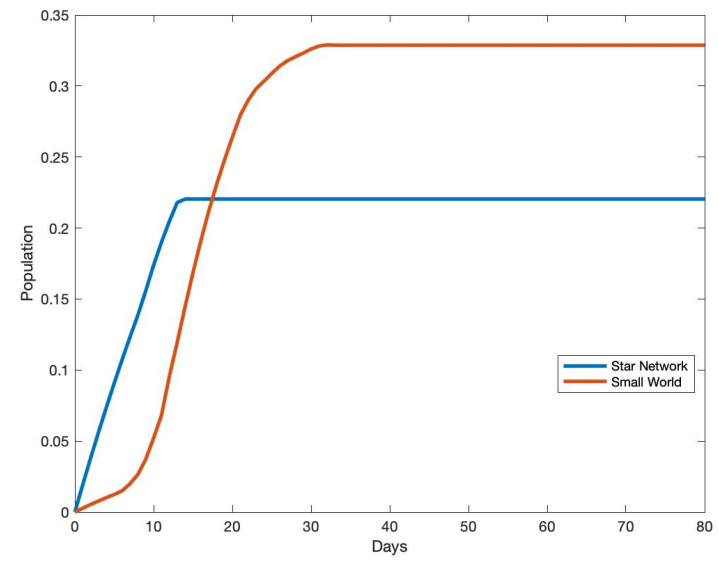

(a) Dynamic of Lockdown

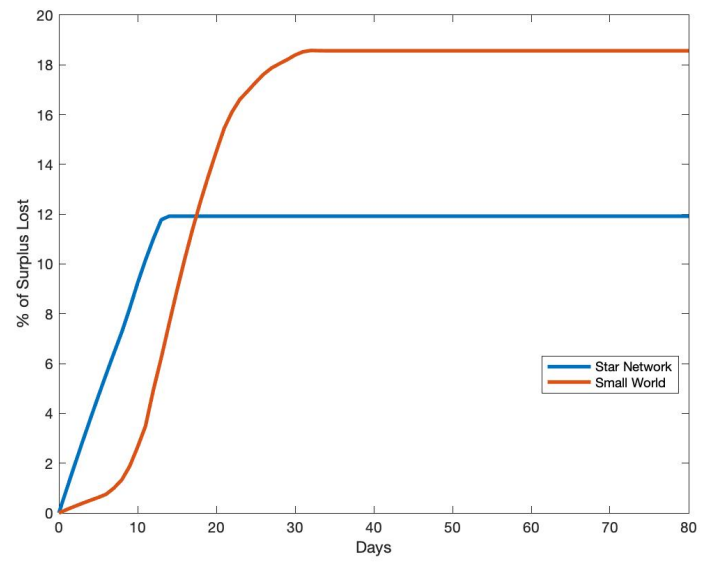

(c) Dynamic of Economic Cost

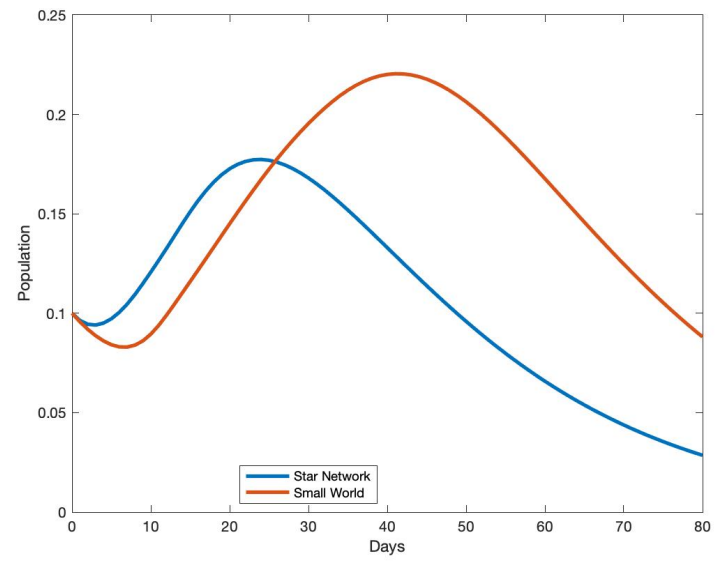

(b) Dynamic of Infection

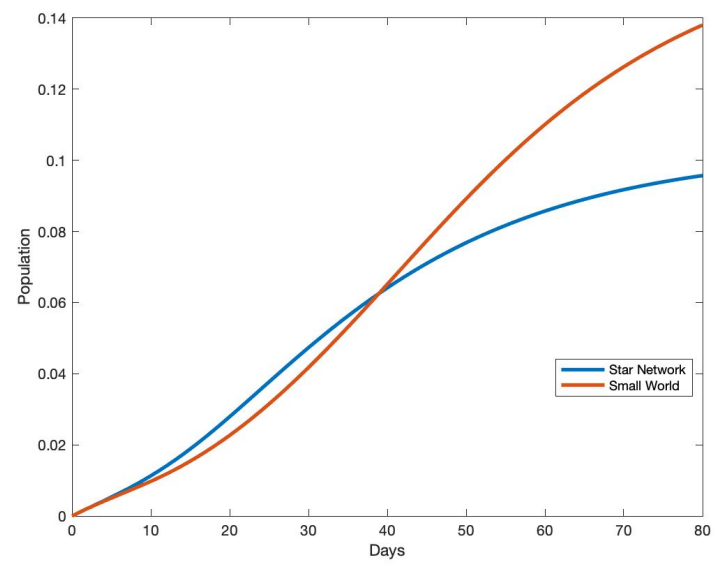

(d) Dynamic of Death

This thought experiment sufficiently highlights the fact that network configuration should be a key factor in the design of optimal lockdown policy during a pandemic like COVID-19, and this 
has important implications for health dynamics and economic costs. Our analysis also suggests that the wide range of variation in COVID-19 outcomes observed across countries and across communities within countries can be explained by differences in their network configuration.

\subsection{Illustration II: Infection Incidence Control and Optimal Lockdown Policy-The Health-vs-Wealth Tradeoff}

In the second illustration of our theoretical findings, we document the effect of varying the tolerated infection incidence level on the optimal lockdown policy and analyze the tradeoff between the desired level of population health and short-term economic gains.

For this analysis, we consider an economy of $n=1000$ agents connected through a small world network with $2 \times n$ edges. This network is simulated using Watts \& Strogatz (1998)'s graph model approach. Figure 4 represents the dynamic of the infection, optimal lockdown, economic costs, and deaths for three different values of infection incidence $\lambda: 0.01,0.05$, and 0.1 .

Figure 4 a illustrates that the optimal cumulative lockdown rate increases with lower infection incidence level. This rate varies from around 6 percent for an incidence level equal to 0.1 to 9 percent for an incidence level of 0.05 to 12 percent for an incidence level of 0.01 . What emerges from these numbers is that the relationship between the tolerated incidence level and the ultimate proportion of the population sent into lockdown is not linear. As the tolerated infection incidence level decreases, the fraction of the population sent into lockdown increases in a proportion that is lower than the decrease.

The optimal lockdown policy resulting from a given tolerated infection incidence level translates into a corresponding dynamic of infection, death and economic cost. In particular, Figure $4 \mathrm{~b}$ shows that a lower tolerated incidence level results in a lower infection and death rate (see Figure $4 \mathrm{~b}$ and Figure 4d).

Figure $4 c$ illustrates the tradeoff between population health and the well-being of the economy. A lower tolerated infection incidence level induces a greater economic cost of the pandemic. Indeed, if the targeted level of infection incidence is low, more individuals must be sent into lockdown. Then, with a decrease in individuals' productiveness in the economy, the loss in terms of economic surplus is huge. For instance, when the tolerated incidence decreases from 0.1 to 
0.05 , the fraction of the economic surplus lost to the pandemic increases from around 3 percent to over 5 percent; and a further decrease of the tolerated incidence level to 0.01 induces an surplus loss of around 6 percent. It follows that a greater level of health is achieved at the expense of short-term economic gains.

Figure 4: Optimal Lockdown Policy and Infection Dynamic

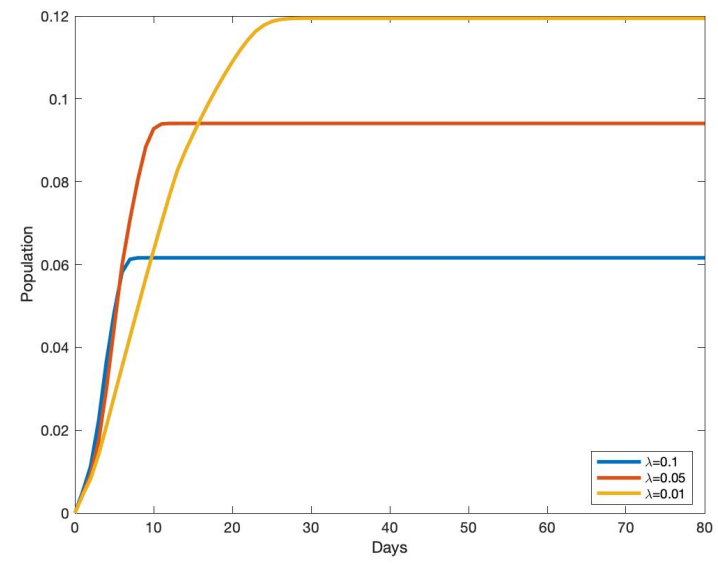

(a) Dynamic of Lockdown

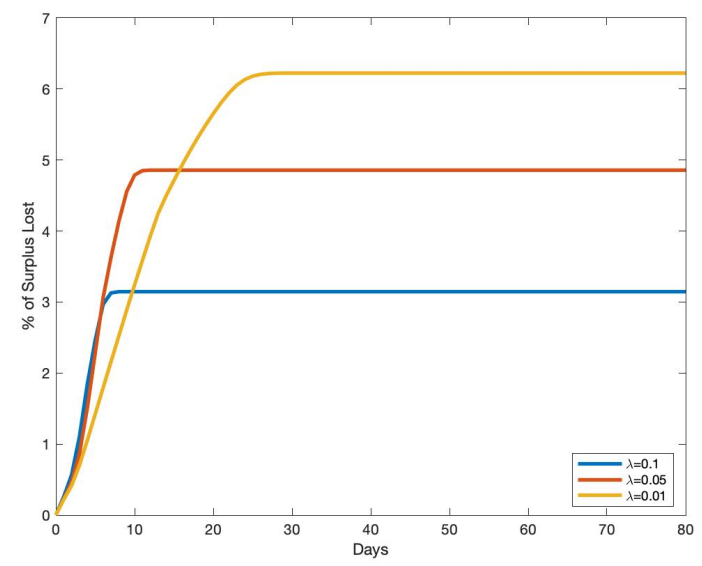

(c) Dynamic of Economic Cost

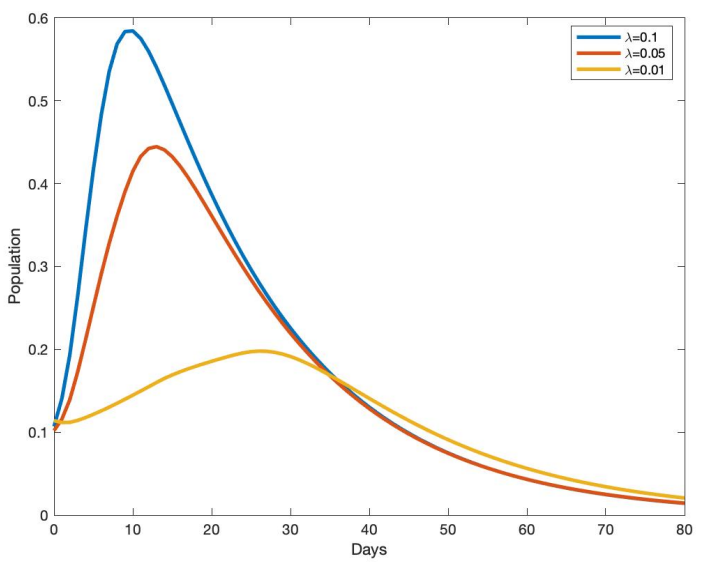

(b) Dynamic of Infection

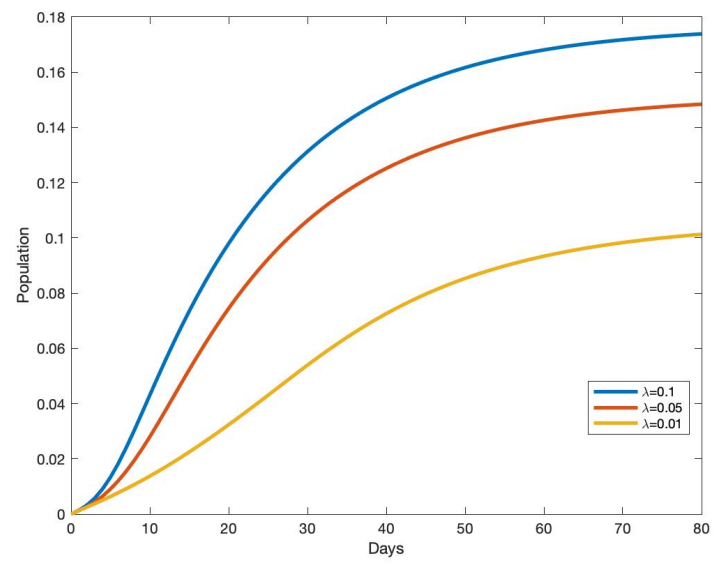

(d) Dynamic of Death 


\subsection{Illustration III: Network Centrality and Optimally Targeted Lock- down}

Our third illustration highlights how lockdown policies can be optimally targeted at individuals based on their characteristics. The individual characteristic we consider is centrality in the contact network. In general, in a networked economy, certain agents occupy more central positions than others in the prevailing contact network. This can be due to a variety of reasons, including the distinct social and economic roles played by each individual. It is argued that individuals who occupy more central positions in networks are more likely to be infected and to spread an infection (see, for example, Pongou \& Tondji (2018) and Rodrigues (2019)). This suggests that an optimal lockdown policy should be targeted at more central agents in a network. However, various measures of network centrality exist, and it is not clear which of these measures are more predictive in the context of a pandemic like COVID-19. Answering this question is essential, and it has important implications for how to optimally design surveys to collect data on social distancing behavior and network structure to curb an infection that spreads through contact networks.

In order to address this question, we consider four popular measures of network centrality: degree centrality, eigenvector centrality, betweenness centrality, and closeness centrality. The degree centrality for a vertex (or agent) in a network is measured by the number of direct connections that vertex has. The betweenness centrality for a vertex is the number of shortest paths that pass through this vertex. It therefore measures the extent to which nodes stand between each other in a network. The eigenvector centrality for a vertex is measured based on relative scores assigned to vertices in a network and reflects the notion that connections to highscoring vertices are more important. For this reason, it is considered a measure of influence or prestige in a network. The closeness centrality for a vertex measures how close this vertex is to all other vertices in a network. It is calculated as the inverse of the sum of the length of the shortest paths between that vertex and all other vertices in a network. It follows from these definitions that degree centrality is less based on network configuration than the other centrality measures.

To answer the question of how each of the aforementioned centrality measures predicts the probability of lockdown, we consider the same economy used to illustrate the health-vs-wealth tradeoff in section 4.2. Agents in this economy are connected through a small world network with 
$2 \times n$ edges. They occupy very distinct positions in this network, resulting in some agents being more central than others. For robustness, our simulation analysis assumes three different values for the tolerated infection incidence $\lambda: 0.01,0.05$, and 0.1 .

Table 1: Correlation between measure of centrality and optimal lockdown probability

\begin{tabular}{ccccccccc}
\hline$\lambda$ & \multicolumn{2}{c}{ Degree } & \multicolumn{2}{c}{ Closeness } & \multicolumn{2}{c}{ Betweness } & \multicolumn{2}{c}{ Eigenvector } \\
\hline & corr & p-value & corr & p-value & corr & p-value & corr & p-value \\
\hline 0.1 & $\mathbf{0 . 3 6}$ & $8 \mathrm{e}-33$ & $\mathbf{0 . 3 4}$ & $9 \mathrm{e}-29$ & $\mathbf{0 . 3 3}$ & $3 \mathrm{e}-27$ & $\mathbf{0 . 2 9}$ & $1 \mathrm{e}-20$ \\
\hline 0.05 & $\mathbf{0 . 2 5}$ & $5 \mathrm{e}-16$ & $\mathbf{0 . 2 1}$ & $1 \mathrm{e}-11$ & $\mathbf{0 . 2 1}$ & $6 \mathrm{e}-12$ & $\mathbf{0 . 1 7}$ & $1 \mathrm{e}-07$ \\
\hline 0.01 & $\mathbf{0 . 2 6}$ & $1 \mathrm{e}-16$ & $\mathbf{0 . 1 8}$ & $4 \mathrm{e}-09$ & $\mathbf{0 . 1 8}$ & $3 \mathrm{e}-09$ & $\mathbf{0 . 1 3}$ & $4 \mathrm{e}-05$
\end{tabular}

The $\mathrm{p}$-value for each centrality measure is for the test of the hypothesis $H_{0} \rho=0$ vs $H_{1} \rho \neq 0$

The results of our analysis are presented in Table 1. This table reports the correlation between each of our centrality measures and optimal lockdown probabilities for different values of the tolerated infection incidence. We find that all of these centrality measures positively correlate to the probability of lockdown under the optimal lockdown policy, and this correlation is statistically significant as implies by the different p-value statistics. However, the predictive value of degree centrality is greater for all values of $\lambda$. These results have at least two practical and/or testable implications.

The first implication is that agents who are more central in the prevailing contact network should have a greater probability of being sent into lockdown. Moreover, in terms of the lockdown timing, more central agents should be confined earlier. Our findings do not imply that all agents should be sent into lockdown, as the goal of lockdown is simply to disconnect the contact network. While quasi-universal lockdown would achieve this goal, it may not be optimal. This finding highlights the limitations of quasi-universal lockdown policies such as those implemented in several countries around the world in the early period of COVID-19.

Our analysis suggests that only particular sectors of society should be shut down during a pandemic like COVID-19. These are sectors that attract large numbers of individuals, such as large shopping centers, airports and other public transportation infrastructures, schools, certain government buildings, entertainment fields, parks, and beaches, among others. Our findings also 
imply that lockdown should be targeted at social and economic hubs, including big cities and high-density areas within a city. Interestingly, this latter policy may be easier to implement in countries with a higher degree of government centralization, which would imply that infection spread is less likely to be contained in less centralized countries as coordination failure among multiple local social planners is likely. This latter prediction can be tested empirically.

Second, our findings have implications for the design of surveys to collect data on social distancing behavior and network structure during a pandemic like COVID-19. Indeed, the fact that degree centrality correlates more highly with the optimal lockdown probability than measures of centrality that are based on network structure means that information on the number of direct connections that an agent has in a network is more relevant for the design of lockdown policies than information on network configuration. Fortunately, information on the number of direct connections or close contacts is also much easier and less costly to collect than information on network configuration, as the latter requires collecting data on people's identity as well as on their direct and indirect contacts. 8

\subsection{Illustration IV: Segregation}

A striking feature of the COVID-19 pandemic is its highly unequal distribution among people of different ethnic and cultural backgrounds.9 In the USA, for example, African-Americans, Indigenous people, Hispanics, and other communities of color have suffered a much greater burden of the pandemic 10 suggesting that both structural and individual factors might be driving its dynamics. We therefore ask the question of whether segregation is a cause of the differential dynamics of COVID-19 outcomes in multi-ethnic societies.

To answer this question, we simulate data to determine the extent to which segregation induces differential COVID-19 outcomes for minority and majority populations. We consider two economies involving a minority ethnic group and a majority ethnic group, and that only differ

\footnotetext{
${ }^{8}$ Information on the number of direct contacts has been collected in some recent surveys on COVID-19 symptoms and social distancing behavior (see, for example, Breza et al. (2020) and Canning et al. (2020)).

${ }^{9}$ See, for example Aldridge et al. (2020), Barr et al. (2020), Cookson \& Milne (2020), Rothschild (2020), and Williamson et al. (2020).

${ }^{10}$ See, for example, U.S. Centers For Disease Control and Prevention (2020), McNeil (2020), APM Research Lab (2020), and Yaya et al. (2020).
} 
Figure 5: Segregated vs Integrated Networks $(n=15)$

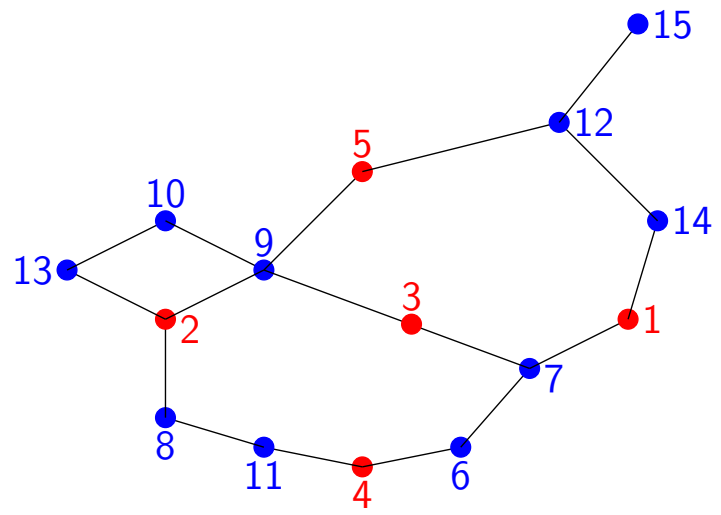

(a) Integrated Network

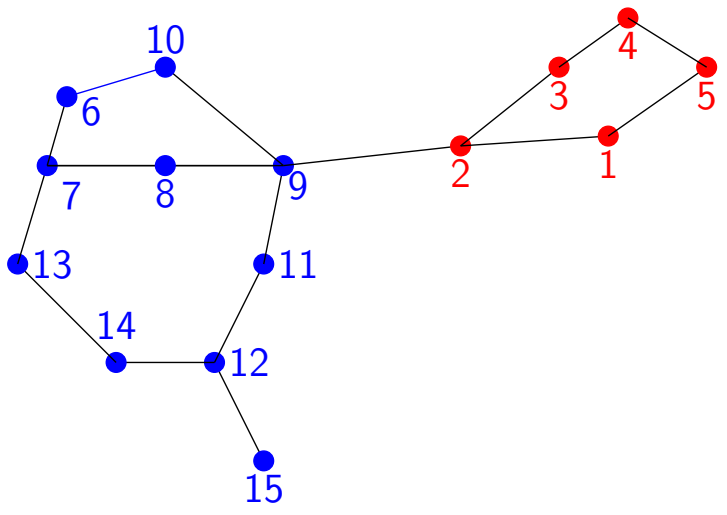

(b) Segregated Network

by their level of ethnic segregation. The first economy, represented by Graph (a) in Figure 5 , is assumed to be integrated, and the second economy, represented by Graph (b) Figure 5 , is assumed to be segregated along ethnic lines. ${ }^{11}$ In simulation, we assume that the incidence level is $\lambda=0.01$. At the initial period, a patient zero is infected. The effects of segregation on minority-majority differences is evaluated by computing the dynamic of the difference-indifferences (diff-in-diffs) estimate, for each outcome of interest. We use the following formula:

$$
D i D_{V, t}=\left[\left(V_{S, m i, t}-V_{I, m i, t}\right)-\left(V_{S, m a, t}-V_{I, m a, t}\right)\right]
$$

where $V \in\{$ Infection, Lockdown, Economist cost, Death $\}, t$ is the day, $S$ represents "segregated", I stands for "integrated", $m i$ for minority group, and ma for majority group. $V_{S, m i, t}$ ( $V_{S, m a, t}$ respectively) is the average probability of the variable of interest $V$ for minority (majority) individuals in the segregated network at time $t$, while $V_{I, m i, t}\left(V_{I, m a, t}\right.$ respectively) is the average probability of the variable of interest $V$ for minority (majority) individuals in the integrated network at time $t$. Below, we represent two typical simulation results from the data, one with individual 3 (from the minority group) as patient zero, and another with individual 11 (member of the majority group) as patient zero. All simulation results are presented in Online

\footnotetext{
${ }^{11}$ We note that these networks are typical representations of integrated and segregated societies. We also assume a small economy (15 agents). This is a deliberate choice that has several advantages. One important advantage is that it allows us to analyze how the identity of patient zero affects outcomes. Working with a large sample size would make this latter analysis intractable.
} 
Supplemental Materials and summarized in the analyses below.

Figure 6: Dynamics of Outcomes under the Optimal Lockdown Policy: Whole Population Patient Zero: Individual 3

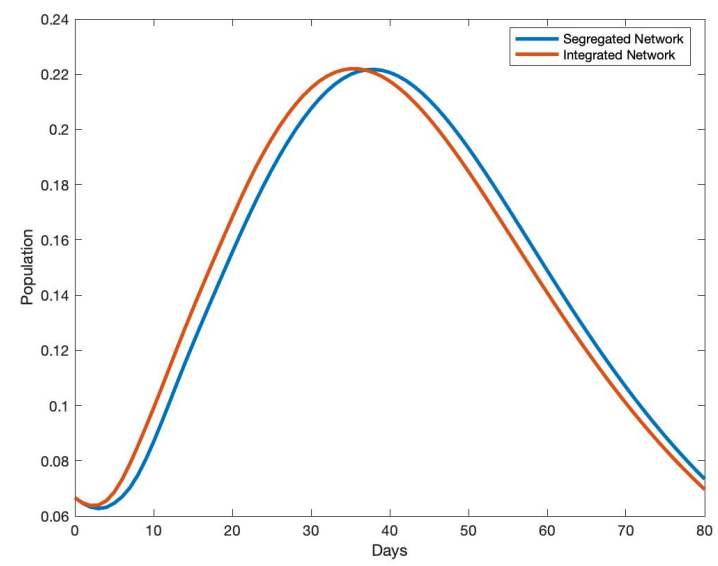

(a) Dynamic of Infection

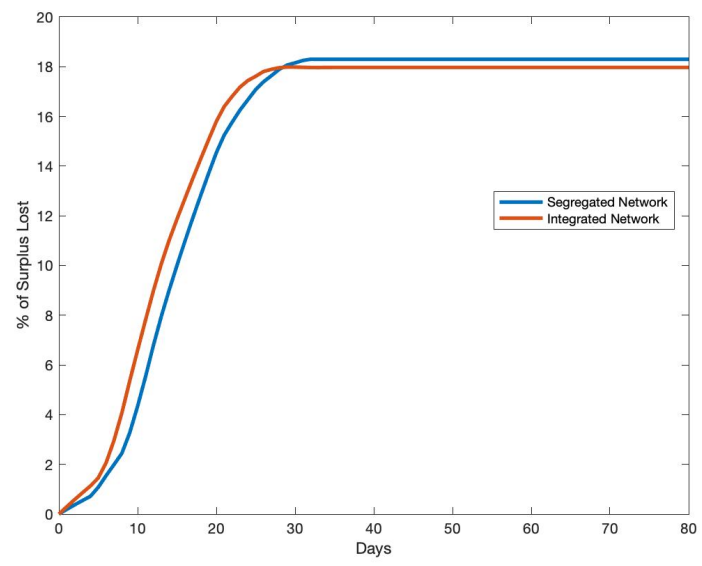

(c) Dynamic of Economic Cost

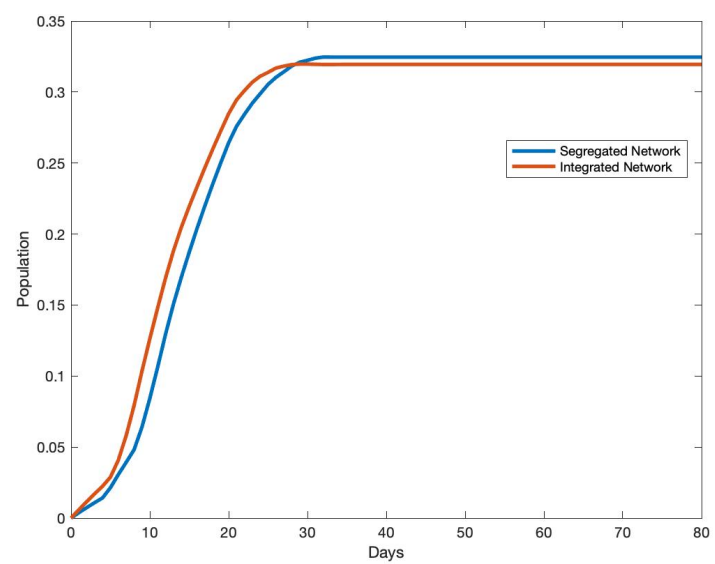

(b) Dynamic of Lockdown

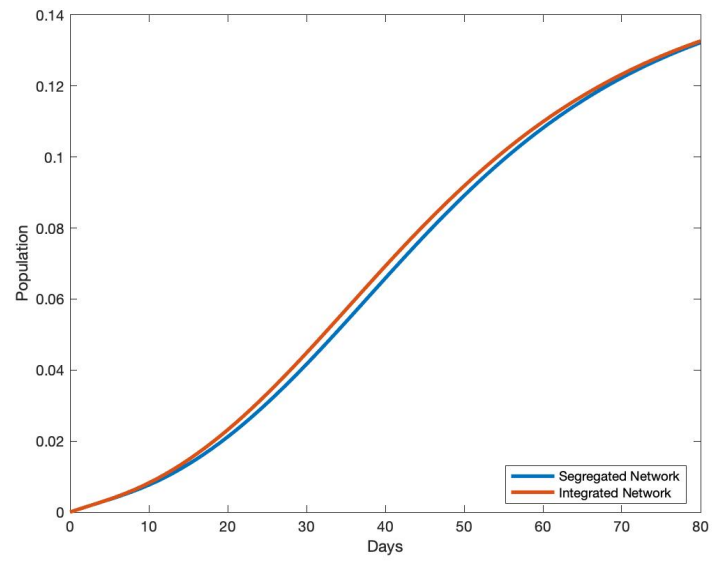

(d) Dynamic of Death

We find that the effect of segregation on outcomes crucially depends on the identity of patient zero. In general, segregation increases lockdown, infection rates and economic losses in the long run when patient zero is from the minority group (Figure 6), but it has the opposite effects when patient zero is from the majority group (Figure 7). However, these effects tend to be small.

Segregation induces significant minority-majority differences in outcomes. The direction of 
Figure 7: Dynamics of Outcomes under the Optimal Lockdown Policy: Whole Population Patient Zero: Individual 11

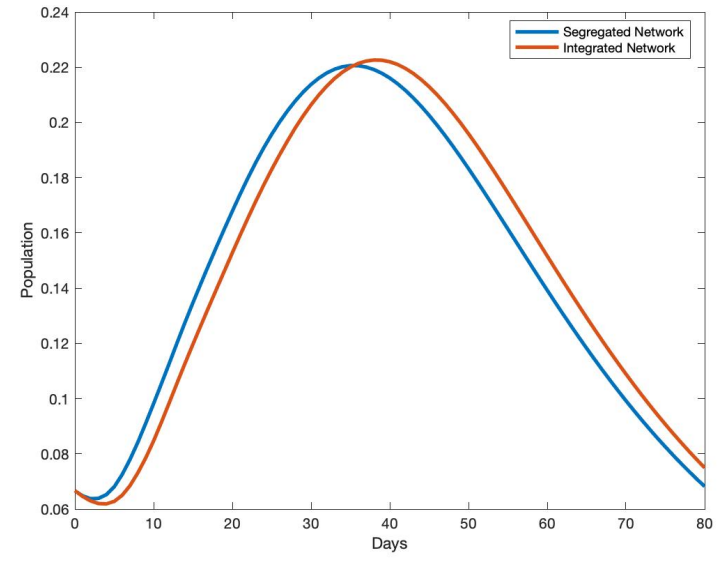

(a) Dynamic of Infection

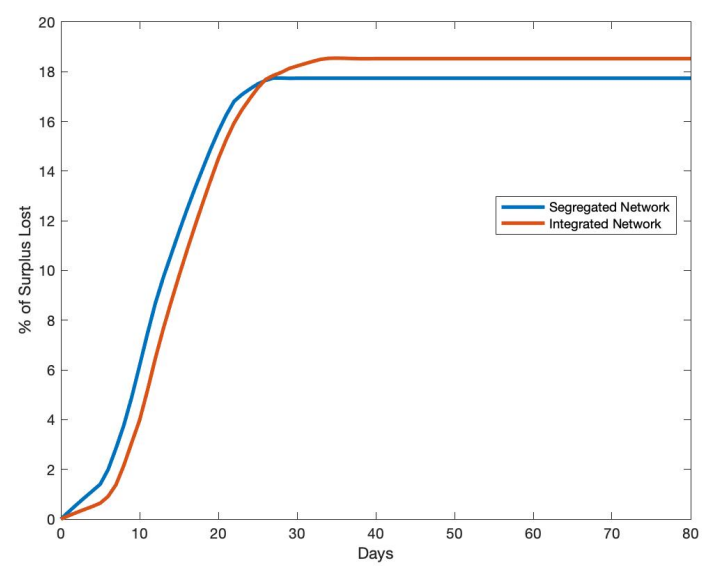

(c) Dynamic of Economic Cost

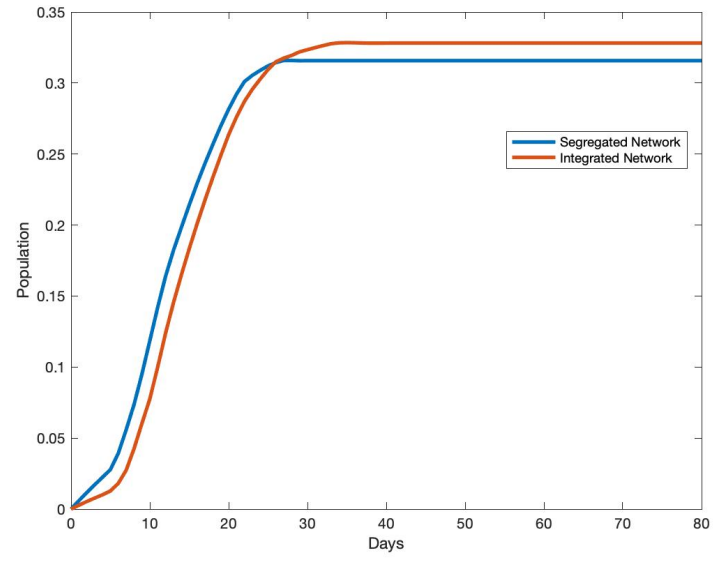

(b) Dynamic of Lockdown

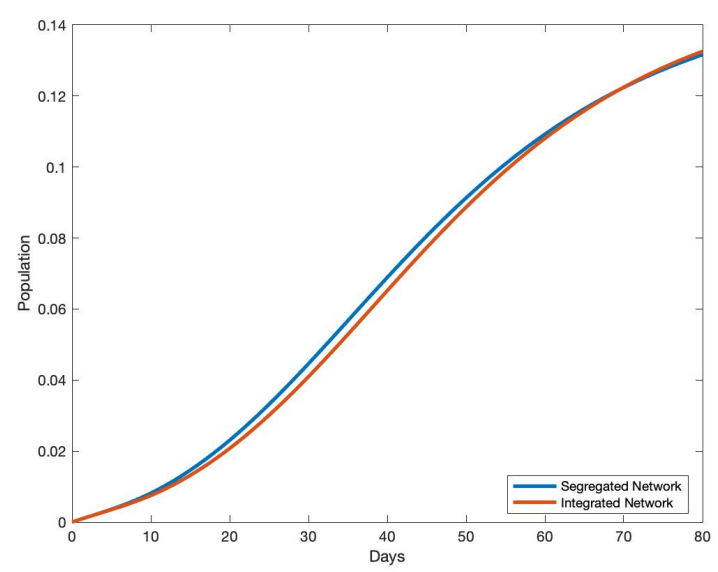

(d) Dynamic of Death

this effect again depends on the identity of patient zero. When patient zero belongs to the majority group (for example, individual 11), segregation generally increases infection and death rates among minorities, resulting in greater lockdown rates and economic surplus losses (see Figure 8). If patient zero is a minority individual instead (for example, individual 3 ), segregation has the opposite effects, resulting in greater health and economic costs in majority individuals (see Figure 9). It follows that segregation carries important cross-group externalities. In the early 
period of the pandemic, individuals who are closer to patient zero are more likely to be infected and to be confined to minimize the spread of the disease under the optimal policy. This results in relatively higher lockdown rates and health and economic costs in the group of patient zero in this early period, but these patterns eventually reverse in the later period as the disease spreads to the other group.

Figure 8: Effect of Segregation on Minority-Majority Differences in Outcomes: Diff-in-Diffs Analysis

\section{Patient Zero: Individual 11}

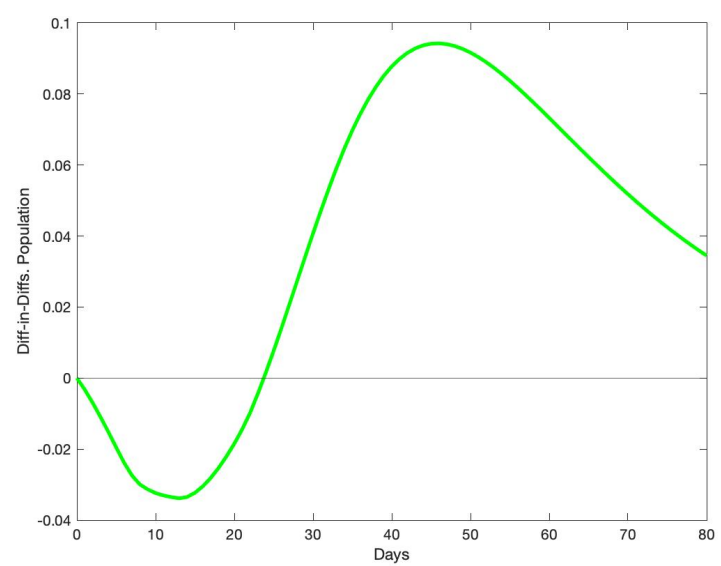

(a) Infection

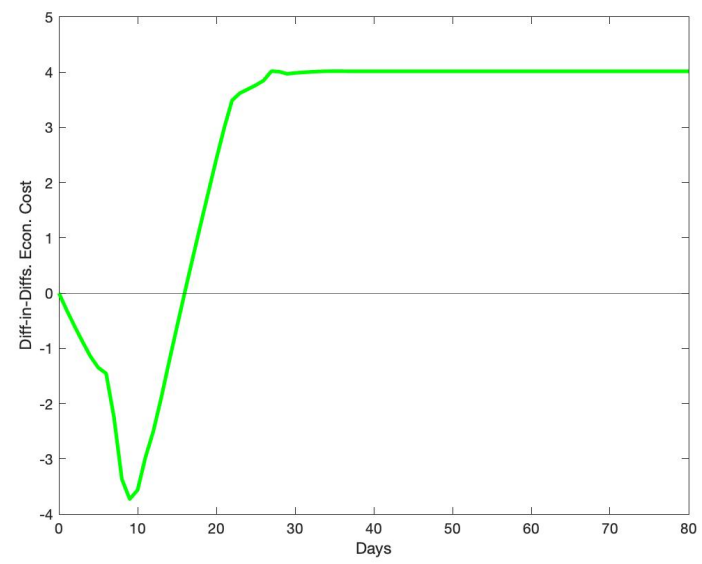

(c) Economic Cost

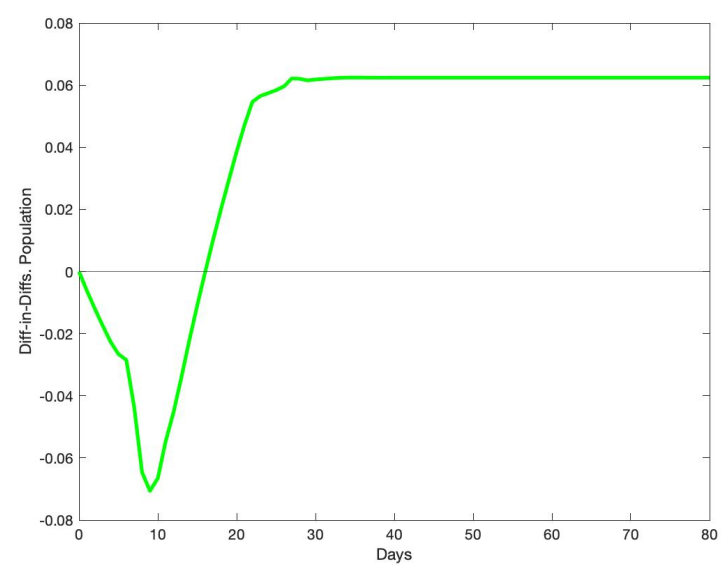

(b) Lockdown

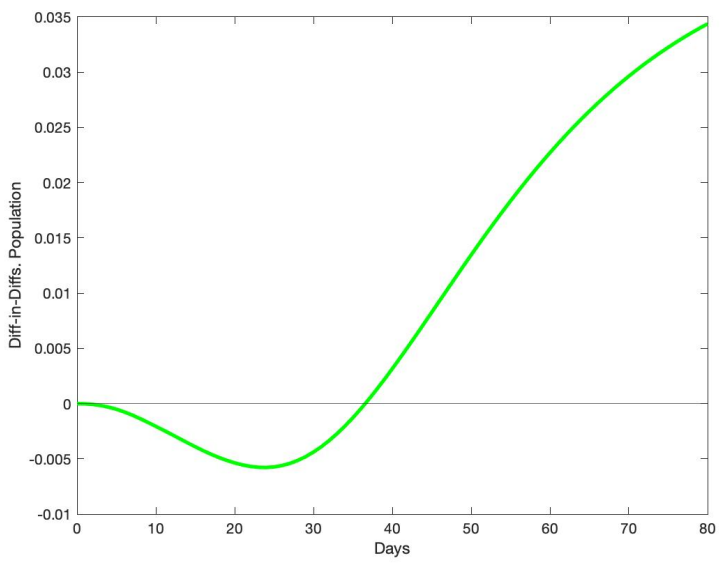

(d) Death

The analysis therefore tends to suggest that if patient zero is chosen randomly, implying that a majority individual is more likely to be the first person to be infected, ethnic segregation is 
Figure 9: Effect of Segregation on Minority-Majority Differences in Outcomes: Diff-in-Diffs Analysis

Patient Zero: Individual 3

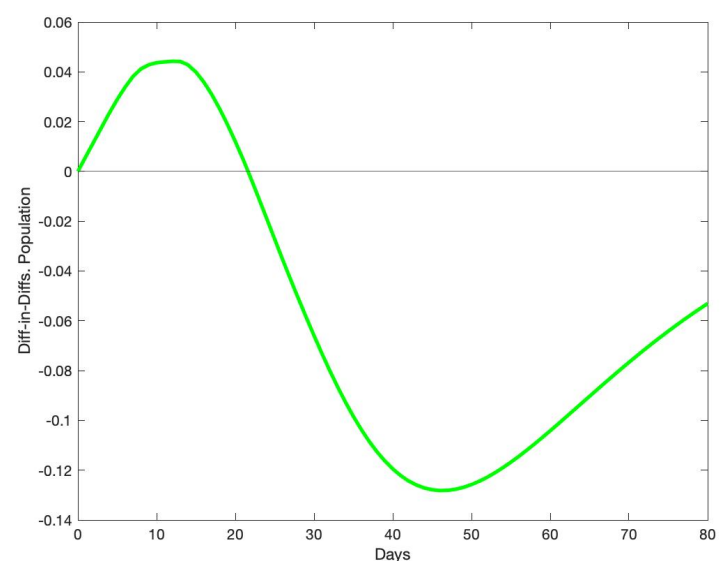

(a) Infection

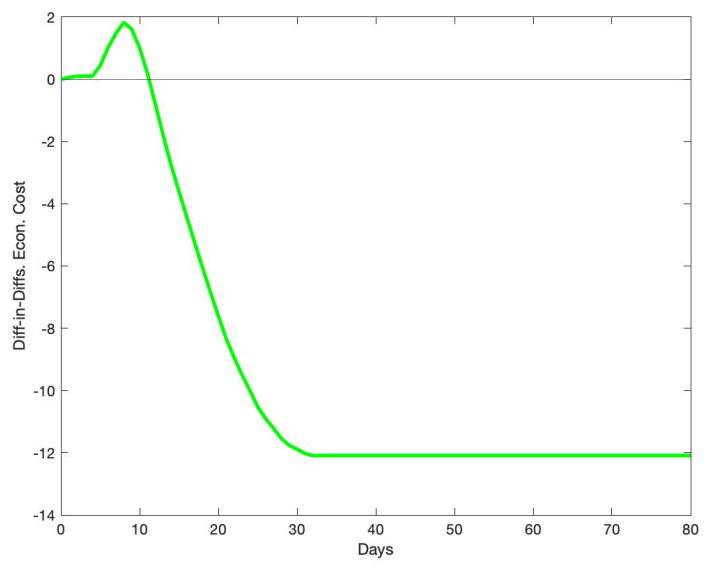

(c) Economic Cost

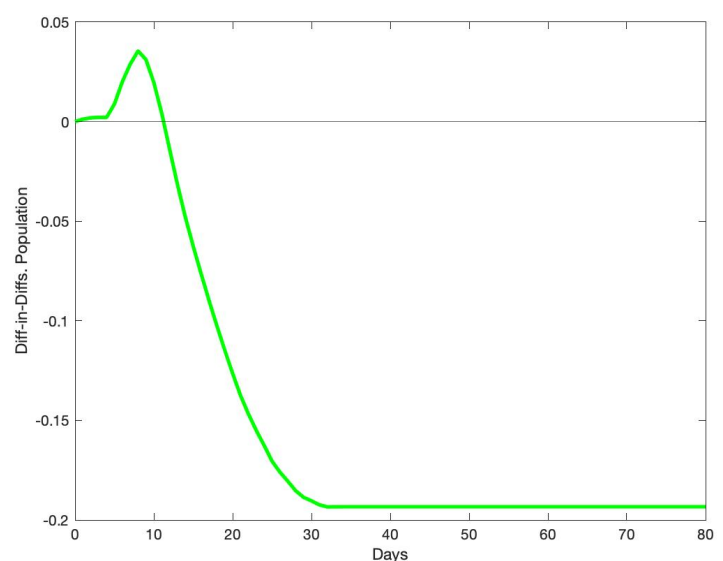

(b) Lockdown

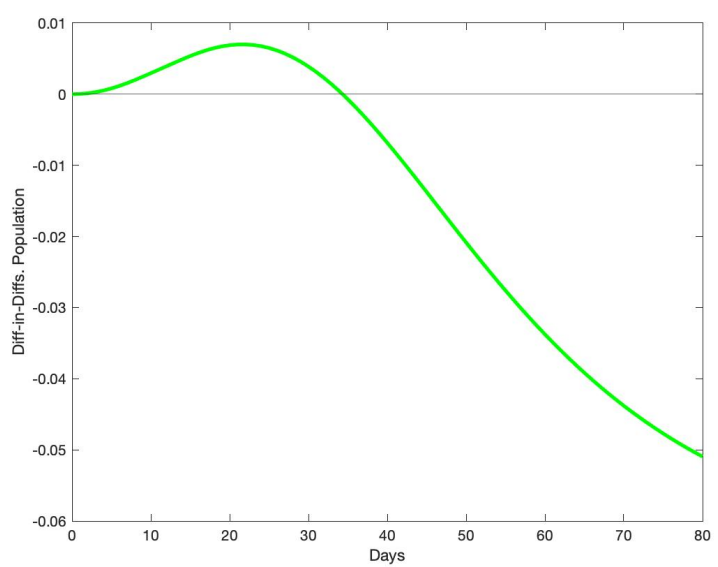

(d) Death

likely to increase infection and death rates among minority populations, also resulting in greater economic costs in this group. It follows that in segregated societies, minority individuals bear a greater health, economic and psychological cost of infections that spread through physical contacts. Our analysis shows a combination of structural and individual factors determines these differential outcomes, and highlights the crucial role of patient 0.12

\footnotetext{
${ }^{12}$ For additional analysis of the optimal lockdown in the presence of a network, we refer interested reader to the Figures 10 to 69 in Online Supplemental Materials.
} 


\section{Conclusion}

This paper solves the problem of finding the optimal lockdown and reopening policy during a pandemic like COVID-19, for a social planner who prioritizes health over short-term economic gains. Agents are connected through a fuzzy network of contacts, and the planner's objective is to determine the policy that contains the spread of infection below a tolerable incidence level and that maximizes the present discounted value of real income, in that order of priority. We show theoretically that the planner's problem has a unique solution. The optimal policy depends both on the configuration of the contact network and the tolerated infection incidence level. Simulation-based comparative statics analyses highlight the crucial role of network structure in infection spread and quantify the tradeoff between the tolerable infection level and human losses on the one hand, and the economic losses due to the pandemic on the other hand.

The simulation exercises also show how different measures of network centrality correlate with the likelihood of being sent into lockdown, showing that degree centrality is more predictive than other measures of centrality. This result has implications for the optimal design of surveys to collect data on social distancing measures and network structure, and suggests that information on direct connections is more relevant than information on the whole configuration of a contact network. This result also suggests that priority in exiting lockdown should be given to professions in which employees have a limited number of contacts.

Moreover, we apply the theoretical findings to analyse how segregation affects health and economic dynamics in minority and majority populations in ethnically segregated societies. We find that while segregation induces differences in the dynamics of infection, death, lockdown, and economic losses, the direction of these differences crucially depends on the identity of patient zero. Our results indicate that segregation results in minorities bearing a greater burden of disease and death, and higher economic costs, during a pandemic like COVID-19 in the majority of scenarios regarding the starting point of the infection. The analysis suggests that a combination of both structural and individual factors explain why minority populations seem to have suffered a greater

burden of COVID-19 in countries like the USA, Canada and the United Kingdom. Importantly, while our main application is to the current COVID-19 crisis, we believe that our model has implications for all other infections that spread through physical contacts. 


\section{References}

Acemoglu, D., Chernozhukov, V., Werning, I., \& Whinston, M. D. (2020). A Multi-Risk SIR Model with Optimally Targeted Lockdown. Working Paper 27102, National Bureau of Economic Research.

Aiello, R. (2020). With pandemic surging globally, PM says health top priority in any border reopening plans. News, CTV News.

Aldridge, R. W., Lewer, D., Katikireddi, S. V., Mathur, R., Pathak, N., Burns, R., Fragaszy, E. B., Johnson, A. M., Devakumar, D., Abubakar, I., et al. (2020). Black, asian and minority ethnic groups in england are at increased risk of death from covid-19: indirect standardisation of nhs mortality data. Wellcome Open Research, 5(88), 88.

Alvarez, F. E., Argente, D., \& Lippi, F. (2020). A simple planning problem for covid-19 lockdown. Technical report, National Bureau of Economic Research.

Anastassopoulou, C., Russo, L., Tsakris, A., \& Siettos, C. (2020). Data-based analysis, modelling and forecasting of the covid-19 outbreak. PloS one, 15(3), e0230405.

APM Research Lab (2020). "the color of coronavirus: COVID-19 deaths by race and ethnicity in the U.S.

Barr, C., Kommenda, N., Mclntyre, N., \& Voce, A. (2020). Ethnic minorities dying of covid-19 at higher rate, analysis shows. The Guardian. Apr, 22.

Bethune, Z. A. \& Korinek, A. (2020). Covid-19 infection externalities: Trading off lives vs. livelihoods. Technical report, National Bureau of Economic Research.

Brauer, F., Castillo-Chavez, C., \& Castillo-Chavez, C. (2012). Mathematical models in population biology and epidemiology, volume 2. Springer. 
Breza, E., Chandrasekhar, A. G., McCormick, T. H., \& Pan, M. (2020). Using aggregated relational data to feasibly identify network structure without network data. American Economic Review, 110(8), 2454-84.

Buchholz, K. (2020). What share of the world population is already on covid-19 lockdown? https://www.statista.com/chart/21240/ enforced-covid-19-lockdowns-by-people-affected-per-country/. Accessed: 2020-05-06.

Canning, D., Karra, M., Dayalu, R., Guo, M., \& Bloom, D. E. (2020). The association between age, covid-19 symptoms, and social distancing behavior in the united states. medRxiv.

Cookson, C. \& Milne, R. (2020). Nations look into why coronavirus hits ethnic minorities so hard. Financial Times.

Debnam Guzman, J., Mabeu, M. C., \& Pongou, R. (2020). Identity During a Pandemic: COVID19 and Ethnic Divisions in the United States. Working paper.

Deji, O. F. (2011). Gender and rural development: Introduction, volume 1. LIT Verlag Münster.

Dingel, J. I. \& Neiman, B. (2020). How Many Jobs Can be Done at Home? Working Paper 26948, National Bureau of Economic Research.

Eichenbaum, M. S., Rebelo, S., \& Trabandt, M. (2020). The macroeconomics of epidemics. Technical report, National Bureau of Economic Research.

Fernández-Villaverde, J. \& Jones, C. M. (2020). Estimating and simulating a SIRD model of covid-19 for many countries, states, and cities.

Harris, J. E. (2020). The Subways Seeded the Massive Coronavirus Epidemic in New York City. Working Paper 27021, National Bureau of Economic Research.

Heap, S. P. H., Koop, C., Matakos, K., Unan, A., \& Weber, N. (2020). Valuating health vs wealth: The effect of information and how this matters for COVID-19 policymaking. Article, VoxEU.org. 
Hethcote, H. W. (2000). The mathematics of infectious diseases. SIAM review, 42(4), 599-653.

John, M. (2020). How is the world dealing with lockdown? This poll has captured the public's mood. Opinion, World Economic Forum.

Kermack, W. O. \& McKendrick, A. G. (1927). A contribution to the mathematical theory of epidemics. Proceedings of the Royal Society of London. Series A, containing papers of a mathematical and physical character, 115(772), 700-721.

Kermack, W. O. \& McKendrick, A. G. (1932). Contributions to the mathematical theory of epidemics. ii.- - the problem of endemicity. Proceedings of the Royal Society of London. Series A, containing papers of a mathematical and physical character, 138(834), 55-83.

Kuchler, T., Russel, D., \& Stroebel, J. (2020). The Geographic Spread of COVID-19 Correlates with Structure of Social Networks as Measured by Facebook. Working Paper 26990, National Bureau of Economic Research.

Lloyd, A. L., Valeika, S., \& Cintrón-Arias, A. (2006). Infection dynamics on small-world networks. Contemporary Mathematics, 410, 209-234.

McNeil, T. (2020). Why People of Color Are Suffering More from COVID-19. Technical report, TuftsNow.

Peng, L., Yang, W., Zhang, D., Zhuge, C., \& Hong, L. (2020). Epidemic analysis of covid-19 in china by dynamical modeling. arXiv preprint arXiv:2002.06563.

Pindyck, R. S. (2020). COVID-19 and the welfare effects of reducing contagion. Technical report, National Bureau of Economic Research.

Pongou, R. (2010). The economics of fidelity in network formation. PhD thesis, Brown University.

Pongou, R. \& Serrano, R. (2013). Fidelity networks and long-run trends in HIV/AIDS gender gaps. American Economic Review, 103(3), 298-302.

Pongou, R. \& Serrano, R. (2016). Volume of trade and dynamic network formation in two-sided economies. Journal of Mathematical Economics, 63, 147-163. 
Pongou, R. \& Tondji, J.-B. (2018). Valuing inputs under supply uncertainty: The bayesian Shapley value. Games and Economic Behavior, 108, 206-224.

Prem, K., Liu, Y., Russell, T. W., Kucharski, A. J., Eggo, R. M., Davies, N., Flasche, S., Clifford, S., Pearson, C. A., Munday, J. D., et al. (2020). The effect of control strategies to reduce social mixing on outcomes of the covid-19 epidemic in wuhan, china: a modelling study. The Lancet Public Health.

Rodrigues, F. A. (2019). Network centrality: an introduction. In A mathematical modeling approach from nonlinear dynamics to complex systems (pp. 177-196). Springer.

Rothschild, R. (2020). The hidden flaw in Sweden's anti-lockdown strategy. the government expects citizens to freely follow its advice-but not all ethnic groups have equal access to expertise. Foreign Policy. April, 21.

Stiglitz, J. (2020). Joseph Stiglitz on priorities for the post-COVID economy. Opinion, World Economic Forum.

U.S. Centers For Disease Control and Prevention (2020). Health Equity Considerations and Racial and Ethnic Minority Groups. Technical report, National Center for Immunization and Respiratory Diseases (NCIRD), Division of Viral Diseases (DVD).

Watts, D. J. \& Strogatz, S. H. (1998). Collective dynamics of 'small-world' networks. nature, 393(6684), 440-442.

Whyte, K. (2020). GDP could contract by 15 to 25 per cent in second quarter. https://www. bls.gov/cps/employment-situation-covid19-faq-march-2020.pdf. Accessed: 202005-06.

Williamson, E., Walker, A. J., Bhaskaran, K. J., Bacon, S., Bates, C., Morton, C. E., Curtis, H. J., Mehrkar, A., Evans, D., Inglesby, P., et al. (2020). Opensafely: factors associated with covid-19-related hospital death in the linked electronic health records of 17 million adult nhs patients. MedRxiv. 
Yaya, S., Yeboah, H., Charles, C. H., Otu, A., \& Labonte, R. (2020). Ethnic and racial disparities in covid-19-related deaths: counting the trees, hiding the forest. BMJ Global Health, 5(6), e002913.

\section{Appendix A: Theoretical derivations for the planning problem}

We recall the planner's problem (23) below:

$$
\begin{array}{ll}
\underset{\left(l_{i}\right)}{\operatorname{Maximize}} & \int_{0}^{\infty} e^{-\delta t} \sum_{i \in N}\left\{p Y_{i}\left(k_{i}, l_{i}, x_{i}, d_{i}\right)-w h\left(l_{i}, x_{i}, d_{i}\right)\right\} d t \\
\text { subject to } & \dot{x}_{i}=\beta\left[1-x_{i}-r_{i}-d_{i}\right]\left(1-l_{i}\right) \sum_{j \neq i}\left[A_{i j}\left(1-l_{j}\right) x_{j}\right]-(\gamma+\kappa) x_{i} \\
& \dot{x}_{i} \leq \lambda, \lambda \geq 0 \\
& \dot{r}_{i}=\gamma x_{i} \\
& \dot{d}_{i}=\kappa x_{i} \\
& \dot{s}_{i}=-\beta\left[1-x_{i}-r_{i}-d_{i}\right]\left(1-l_{i}\right) \sum_{j \neq i}\left[A_{i j}\left(1-l_{j}\right) x_{j}\right] \\
& X_{i}(0)=\left(s_{i}(0), x_{i}(0), r_{i}(0), d_{i}(0)\right)^{T} \text { given, with } \\
& s_{i}(0)+x_{i}(0)+r_{i}(0)+d_{i}(0)=1
\end{array}
$$

We denote $f_{i}\left(k_{i}, l_{i}, x_{i}, d_{i}\right)=\beta\left[1-x_{i}-r_{i}-d_{i}\right]\left(1-l_{i}\right) \sum_{j \neq i}\left[A_{i j}\left(1-l_{j}\right) x_{j}\right]-(\gamma+\kappa) x_{i}$, and $W_{i}^{p}\left(k_{i}, l_{i}, x_{i}, d_{i}\right)=p Y_{i}\left(k_{i}, l_{i}, x_{i}, d_{i}\right)-w h\left(l_{i}, x_{i}, d_{i}\right)$. The current Hamiltonian of problem (23) is given as:

$$
\begin{array}{r}
\mathcal{H}_{c}\left(l, x, r, d, s, \mu^{1}, \mu^{2}, \mu^{3}, \mu^{4}\right)=\sum_{i \in N} W_{i}^{p}\left(k_{i}, l_{i}, x_{i}, d_{i}\right)+\sum_{i \in N} \mu_{i}^{1} f_{i}+\gamma \sum_{i \in N} \mu_{i}^{2} x_{i}+\kappa \sum_{i \in N} \mu_{i}^{3} x_{i} \\
+\sum_{i \in N} \mu_{i}^{4}\left[-f_{i}-(\gamma+\kappa) x_{i}\right] .
\end{array}
$$

Given the inequality constraints (17), we can augment the current Hamiltonian (29) into a Lagrangian function:

$$
\begin{aligned}
\mathcal{L}\left(l, x, r, d, s, \mu^{1}, \mu^{2}, \mu^{3}, \mu^{4}, \theta\right)=\sum_{i \in N} W_{i}^{p}\left(k_{i}, l_{i}, x_{i}, d_{i}\right) & +\sum_{i \in N} \mu_{i}^{1} f_{i}+\gamma \sum_{i \in N} \mu_{i}^{2} x_{i}+\kappa \sum_{i \in N} \mu_{i}^{3} x_{i} \\
& +\sum_{i \in N} \mu_{i}^{4}\left[-f_{i}-(\gamma+\kappa) x_{i}\right]+\sum_{i \in N} \theta_{i}\left(\lambda-f_{i}\right) .
\end{aligned}
$$


We can also rewrite 30 as:

$$
\begin{array}{r}
\mathcal{L}\left(l, x, r, d, s, \mu^{1}, \mu^{2}, \mu^{3}, \mu^{4}, \theta\right)=\sum_{i \in N} W_{i}^{p}\left(k_{i}, l_{i}, x_{i}, d_{i}\right)+\sum_{i \in N}\left(\mu_{i}^{1}-\mu_{i}^{4}-\theta_{i}\right) f_{i}+\gamma \sum_{i \in N} \mu_{i}^{2} x_{i} \\
+\kappa \sum_{i \in N} \mu_{i}^{3} x_{i}-(\gamma+\kappa) \sum_{i \in N} \mu_{i}^{4} x_{i}+\lambda \sum_{i \in N} \theta_{i}
\end{array}
$$

The first-order conditions for maximizing $\mathcal{L}$ call for, assuming interior solutions,

$$
\frac{\partial \mathcal{L}}{\partial l_{k}}=0, k \in N
$$

as well as

$$
\frac{\partial \mathcal{L}}{\partial \theta_{k}}=\lambda-\dot{x_{k}} \geq 0, \theta_{k} \geq 0, \theta_{k} \frac{\partial \mathcal{L}}{\partial \theta_{k}}=\theta_{k}\left(\lambda-\dot{x_{k}}\right)=0, k \in N
$$

Finally, the other maximum-principle conditions that include the dynamics for state and co-state variables are, for $k \in N$ :

$$
\begin{aligned}
& \dot{x_{k}}=\frac{\partial \mathcal{L}}{\partial \mu_{k}^{1}} \quad \quad \dot{r}_{k}=\frac{\partial \mathcal{L}}{\partial \mu_{k}^{2}} \quad \dot{d}_{k}=\frac{\partial \mathcal{L}}{\partial \mu_{k}^{3}} \quad \dot{s_{k}}=\frac{\partial \mathcal{L}}{\partial \mu_{k}^{4}} \\
& \dot{\mu_{k}^{1}}=\delta \mu_{k}^{1}-\frac{\partial \mathcal{L}}{\partial x_{k}} \quad \dot{\mu_{k}^{2}}=\delta \mu_{k}^{2}-\frac{\partial \mathcal{L}}{\partial r_{k}} \quad \dot{\mu_{k}^{3}}=\delta \mu_{k}^{3}-\frac{\partial \mathcal{L}}{\partial d_{k}} \quad \dot{\mu_{k}^{4}}=\delta \mu_{k}^{4}-\frac{\partial \mathcal{L}}{\partial s_{k}}
\end{aligned}
$$

Recall that $f_{i}\left(l_{i}, x_{i}, r_{i}, d_{i}\right)=\beta\left(1-x_{i}-r_{i}-d_{i}\right)\left(1-l_{i}\right) \sum_{j \neq i}\left[A_{i j}\left(1-l_{j}\right) x_{j}\right]-(\gamma+\kappa) x_{i}$. Then,

$$
\begin{aligned}
\frac{\partial f_{i}}{\partial l_{k}}= \begin{cases}-\beta\left(1-x_{i}-r_{i}-d_{i}\right) \sum_{j \neq i}\left[A_{i j}\left(1-l_{j}\right) x_{j}\right] & \text { if } k=i \\
-\beta\left(1-x_{i}-r_{i}-d_{i}\right)\left(1-l_{i}\right) A_{i k} x_{k} & \text { if } k \neq i\end{cases} \\
\frac{\partial f_{i}}{\partial x_{k}}= \begin{cases}-\beta\left(1-l_{i}\right) \sum_{j \neq i}\left[A_{i j}\left(1-l_{j}\right) x_{j}\right]-(\gamma+\kappa) & \text { if } k=i \\
\beta\left(1-x_{i}-r_{i}-d_{i}\right)\left(1-l_{i}\right)\left(1-l_{k}\right) A_{i k} & \text { if } k \neq i\end{cases} \\
\frac{\partial f_{i}}{\partial r_{k}}=\frac{\partial f_{i}}{\partial d_{k}}= \begin{cases}-\beta\left(1-l_{i}\right) \sum_{j \neq i}\left[A_{i j}\left(1-l_{j}\right) x_{j}\right] & \text { if } k=i \\
0 & \text { if } k \neq i\end{cases}
\end{aligned}
$$


We also recall that $W_{i}^{p}\left(k_{i}, l_{i}, x_{i}, d_{i}\right)=p Y_{i}\left(k_{i}, l_{i}, x_{i}, d_{i}\right)-w h\left(l_{i}, x_{i}, d_{i}\right)$. Therefore,

$$
\begin{aligned}
& \frac{\partial W_{i}^{p}}{\partial l_{k}}= \begin{cases}p \frac{\partial Y_{i}}{\partial l_{k}}-w \frac{\partial h}{\partial l_{k}} & \text { if } k=i \\
0 & \text { if } k \neq i\end{cases} \\
& \frac{\partial W_{i}^{p}}{\partial x_{k}}= \begin{cases}p \frac{\partial Y_{i}}{\partial x_{k}}-w \frac{\partial h}{\partial x_{k}} & \text { if } k=i \\
0 & \text { if } k \neq i\end{cases} \\
& \frac{\partial W_{i}^{p}}{\partial r_{k}}=0 \\
& \frac{\partial W_{i}^{p}}{\partial d_{k}}= \begin{cases}p \frac{\partial Y_{i}}{\partial d_{k}}-w \frac{\partial h}{\partial d_{k}} & \text { if } k=i \\
0 & \text { if } k \neq i\end{cases}
\end{aligned}
$$

Therefore, using (33), we have the following equation:

$$
\begin{aligned}
\frac{\partial \mathcal{L}}{\partial l_{k}} & =\sum_{i \in N} \frac{\partial W_{i}^{p}}{\partial l_{k}}+\sum_{i \in N}\left(\mu_{i}^{1}-\mu_{i}^{4}-\theta_{i}\right) \frac{\partial f_{i}}{\partial l_{k}} \\
& =\frac{\partial W_{k}^{p}}{\partial l_{k}}+\sum_{i \in N}\left(\mu_{i}^{1}-\mu_{i}^{4}-\theta_{i}\right) \frac{\partial f_{i}}{\partial l_{k}} \text { using } 39 \\
& =p \frac{\partial Y_{k}}{\partial l_{k}}-w \frac{\partial h}{\partial l_{k}}+\sum_{i \in N}\left(\mu_{i}^{1}-\mu_{i}^{4}-\theta_{i}\right) \frac{\partial f_{i}}{\partial l_{k}}
\end{aligned}
$$

Hence, using the first-order conditions (32), equation (43) becomes:

$$
0=p \frac{\partial Y_{k}}{\partial l_{k}}-w \frac{\partial h}{\partial l_{k}}+\sum_{i \in N}\left(\mu_{i}^{1}-\mu_{i}^{4}-\theta_{i}\right) \frac{\partial f_{i}}{\partial l_{k}}
$$

Using the other conditions from (35) and using (40):

$$
\dot{\mu_{k}^{1}}=\delta \mu_{k}^{1}-\frac{\partial \mathcal{L}}{\partial x_{k}}=\delta \mu_{k}^{1}-p \frac{\partial Y_{k}}{\partial x_{k}}+w \frac{\partial h}{\partial x_{k}}-\gamma \mu_{k}^{2}-\kappa \mu_{k}^{3}+(\gamma+\kappa) \mu_{k}^{4}-\sum_{i \in N}\left(\mu_{i}^{1}-\mu_{i}^{4}-\theta_{i}\right) \frac{\partial f_{i}}{\partial x_{k}} .
$$

Similarly, using (35), we get:

$$
\begin{gathered}
\left.\dot{\mu_{k}^{2}}=\delta \mu_{k}^{2}-\frac{\partial \mathcal{L}}{\partial r_{k}}=\delta \mu_{k}^{2}-\sum_{i \in N}\left(\mu_{i}^{1}-\mu_{i}^{4}-\theta_{i}\right) \frac{\partial f_{i}}{\partial r_{k}} \text { using } \sqrt[41)\right]{ } \\
\dot{\mu_{k}^{3}}=\delta \mu_{k}^{3}-\frac{\partial \mathcal{L}}{\partial d_{k}}=\delta \mu_{k}^{3}-p \frac{\partial Y_{k}}{\partial d_{k}}+w \frac{\partial h}{\partial d_{k}}-\sum_{i \in N}\left(\mu_{i}^{1}-\mu_{i}^{4}-\theta_{i}\right) \frac{\partial f_{i}}{\partial d_{k}} \text { using }
\end{gathered}
$$

and

$$
\dot{\mu_{k}^{4}}=\delta \mu_{k}^{4}-\frac{\partial \mathcal{L}}{\partial s_{k}}=\delta \mu_{k}^{4}, \text { since } \frac{\partial \mathcal{L}}{\partial s_{k}}=0
$$




\section{Online Supplemental Materials}

\section{Integrated vs. Segregated Networks Simulation Results}

Figure 10: Dynamics of Outcomes under the Optimal Lockdown Policy: Whole Population

Patient Zero: Individual 1

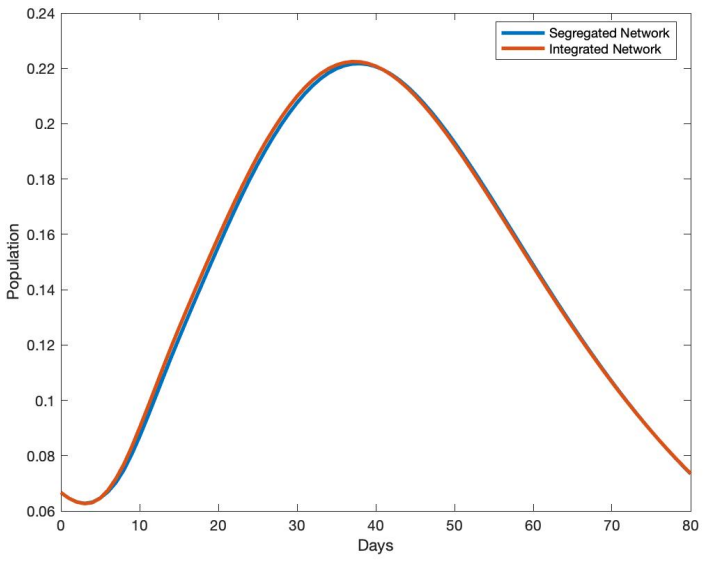

(a) Dynamic of Infection

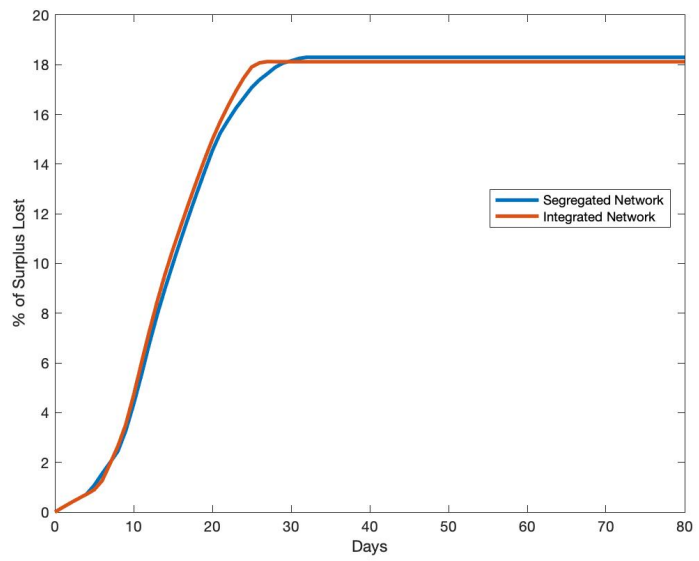

(c) Dynamic of Economic Cost

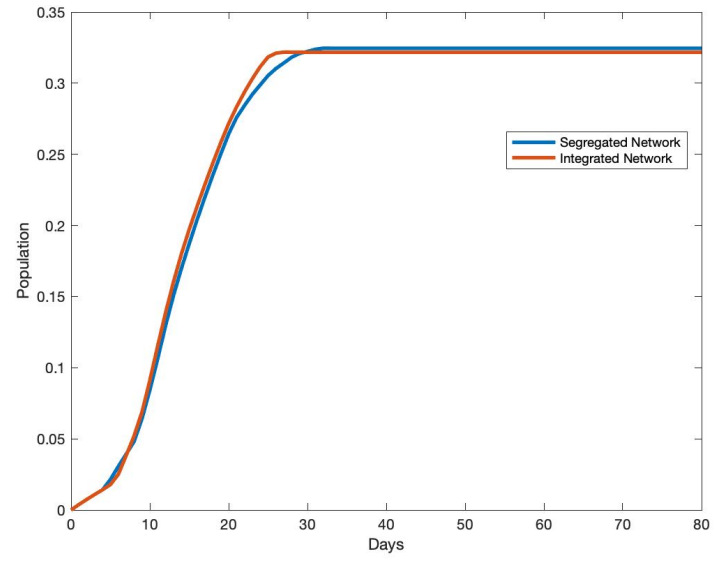

(b) Dynamic of Lockdown

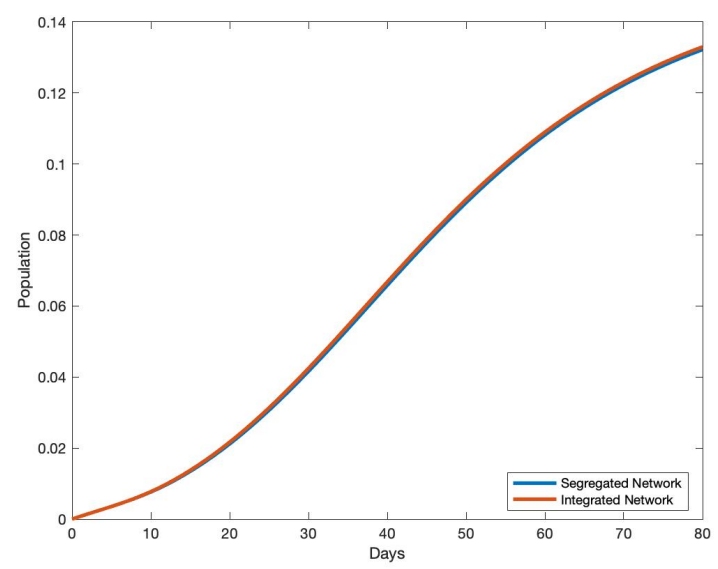

(d) Dynamic of Death 
Figure 11: Dynamics of Outcomes under the Optimal Lockdown Policy: Minority Group (1 to 5) Patient Zero: Individual 1

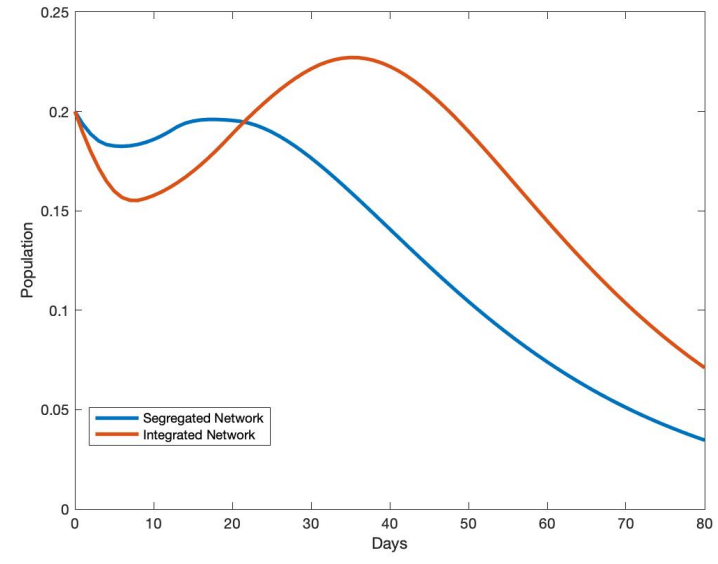

(a) Dynamic of Infection

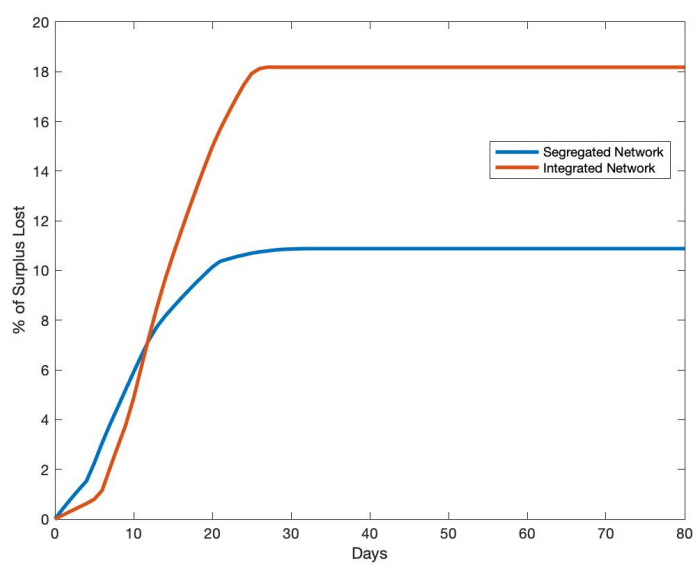

(c) Dynamic of Economic Cost

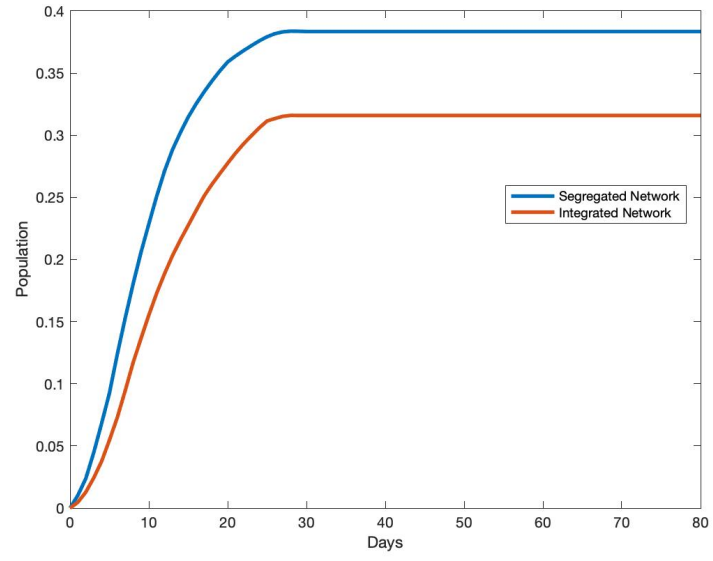

(b) Dynamic of Lockdown

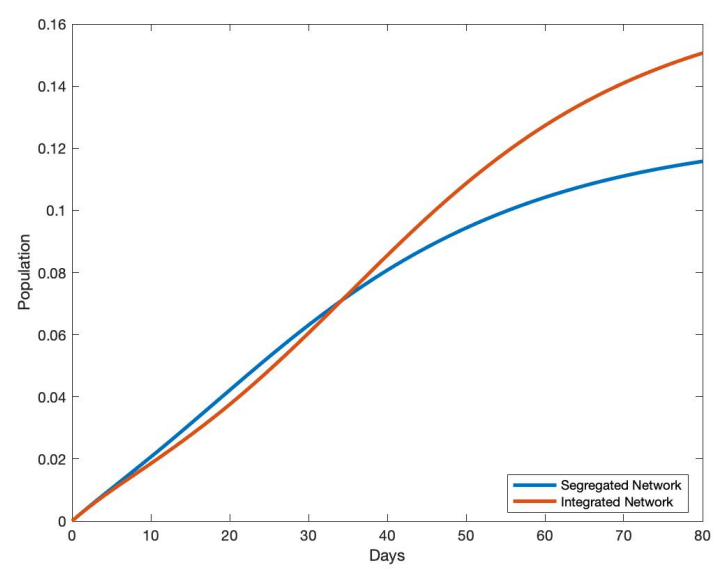

(d) Dynamic of Death 
Figure 12: Dynamics of Outcomes under the Optimal Lockdown Policy: Majority Group (6 to 15)

Patient Zero: Individual 1

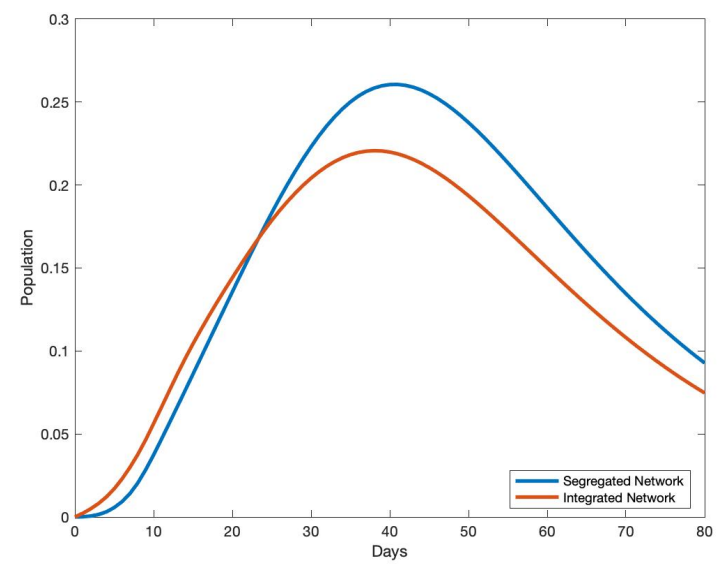

(a) Dynamic of Infection

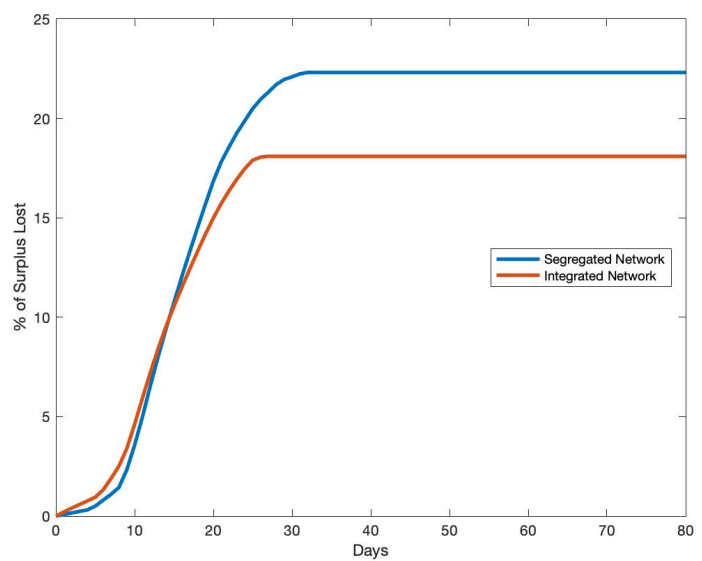

(c) Dynamic of Economic Cost

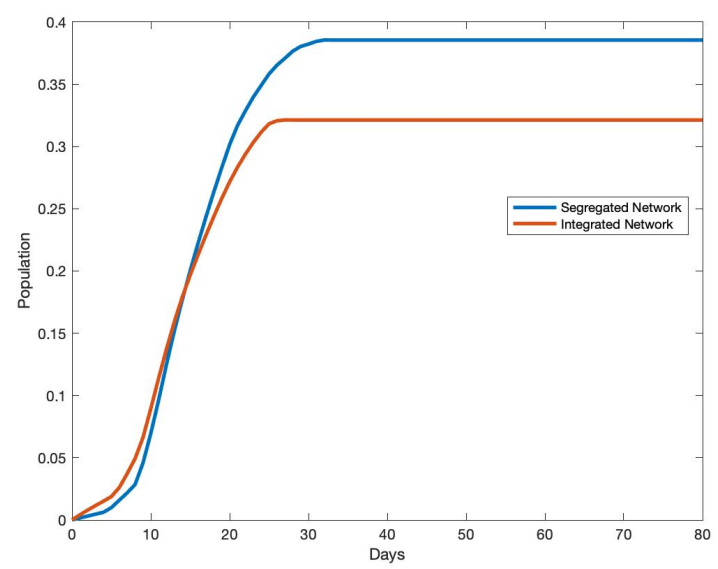

(b) Dynamic of Lockdown

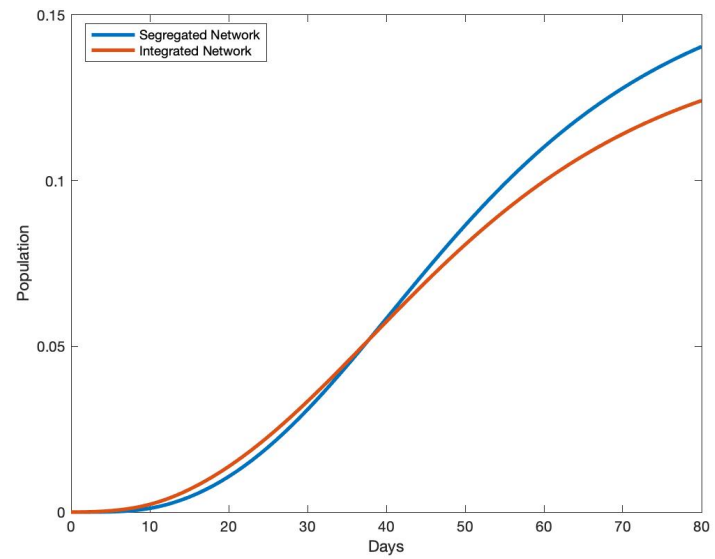

(d) Dynamic of Death 
Figure 13: Effect of Segregation on Minority-Majority Differences in Outcomes: Diff-in-Diffs Analysis

Patient Zero: Individual 1

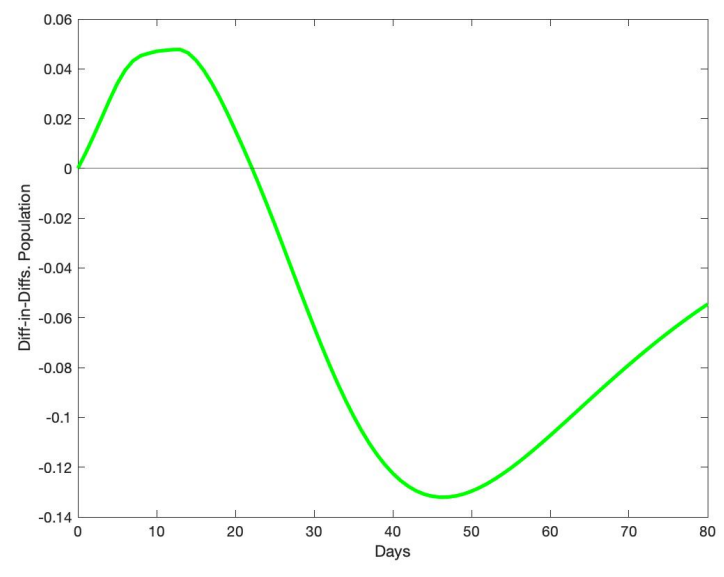

(a) Infection

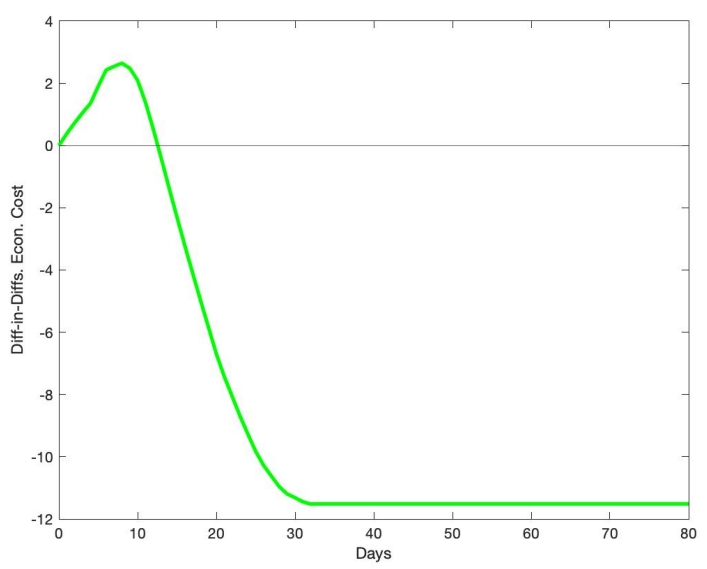

(c) Economic Cost

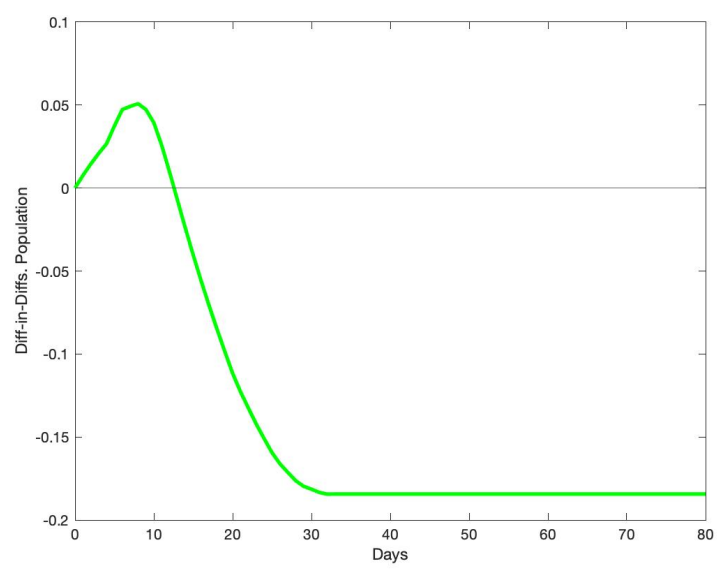

(b) Lockdown

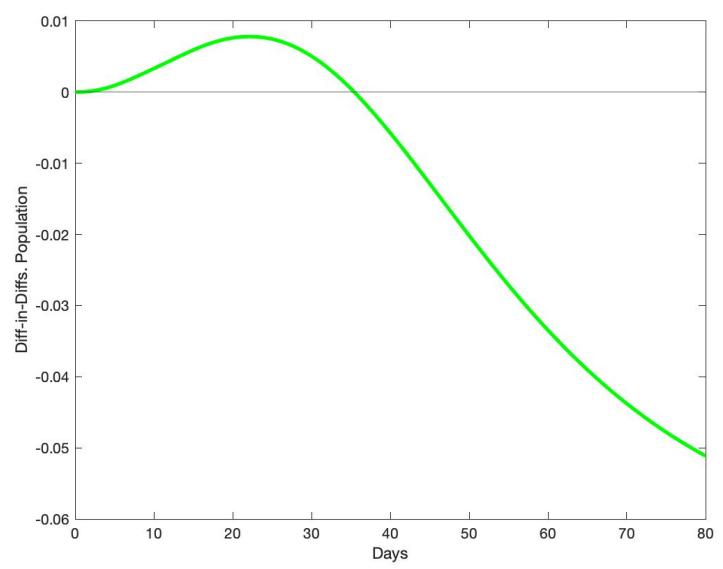

(d) Death 
Figure 14: Dynamics of Outcomes under the Optimal Lockdown Policy: Whole Population Patient Zero: Individual 2

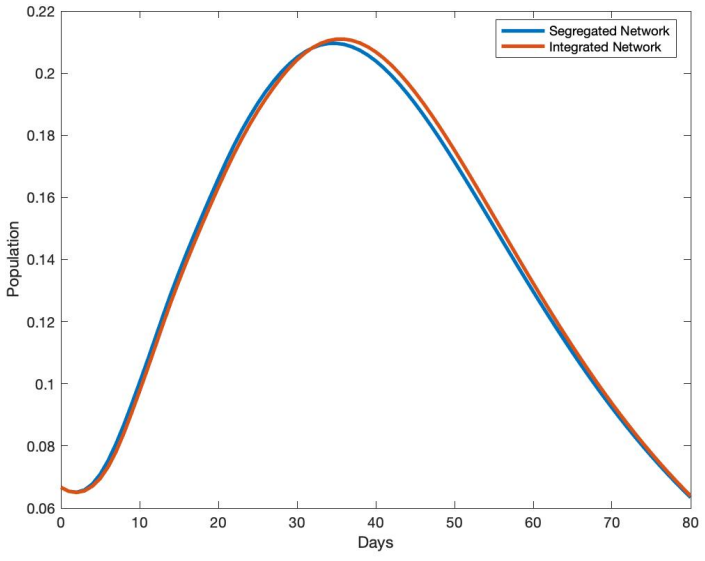

(a) Dynamic of Infection

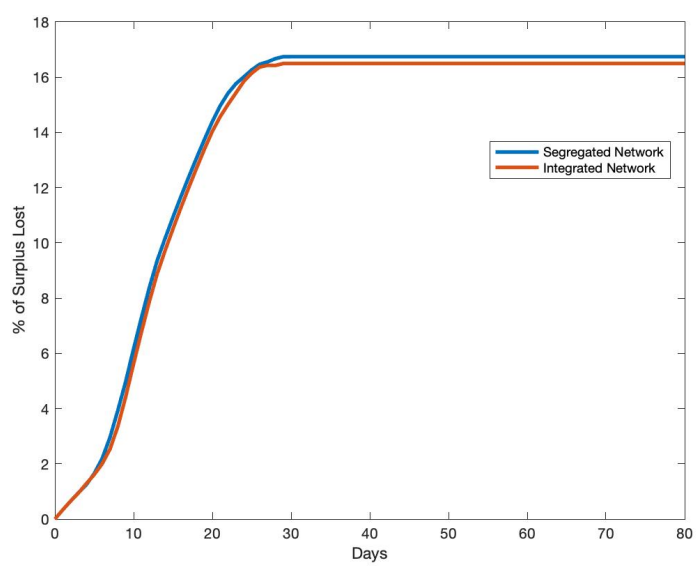

(c) Dynamic of Economic Cost

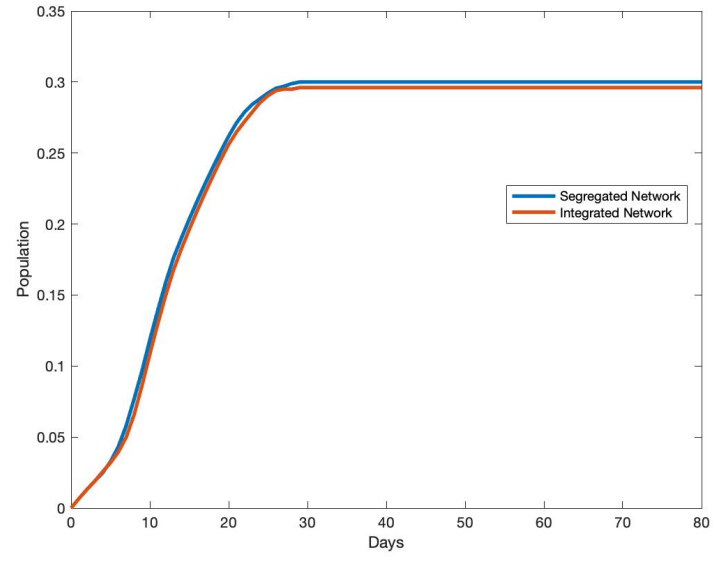

(b) Dynamic of Lockdown

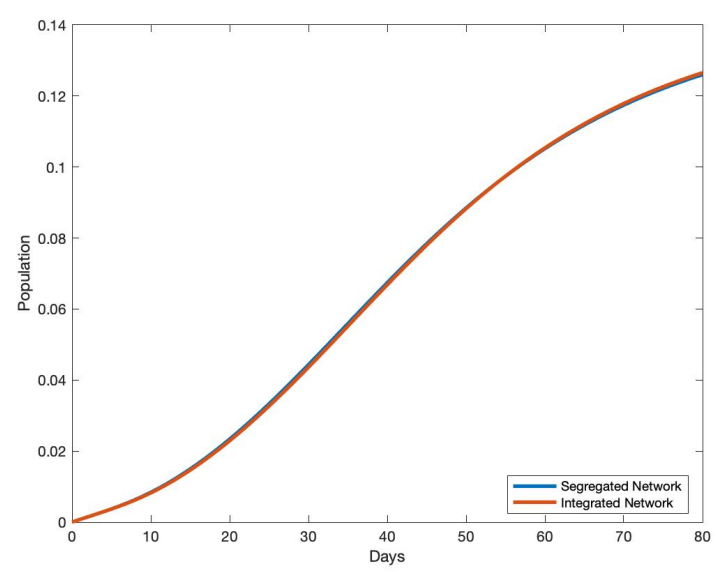

(d) Dynamic of Death 
Figure 15: Dynamics of Outcomes under the Optimal Lockdown Policy: Minority Group (1 to 5)

Patient Zero: Individual 2

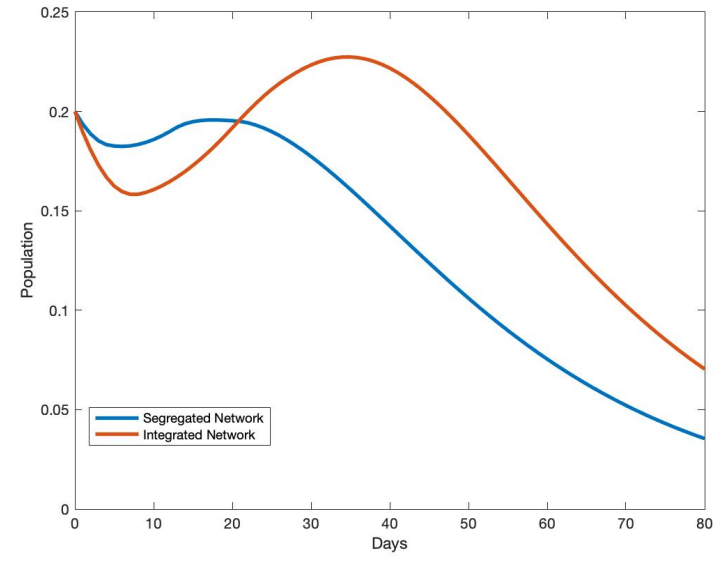

(a) Dynamic of Infection

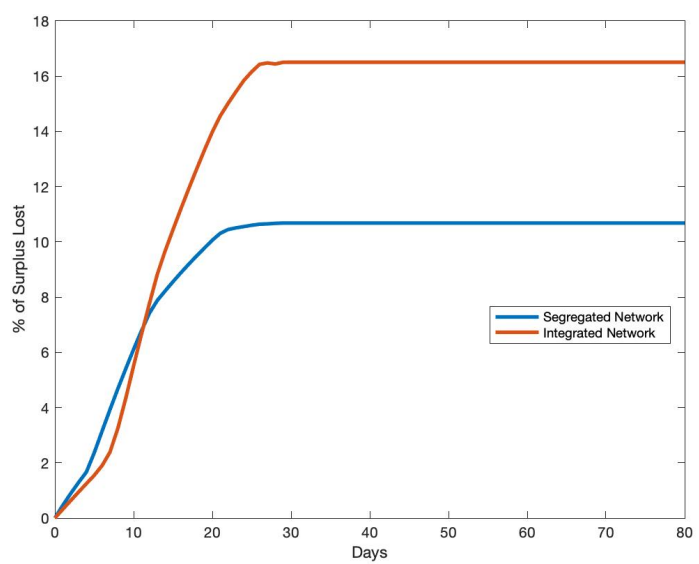

(c) Dynamic of Economic Cost

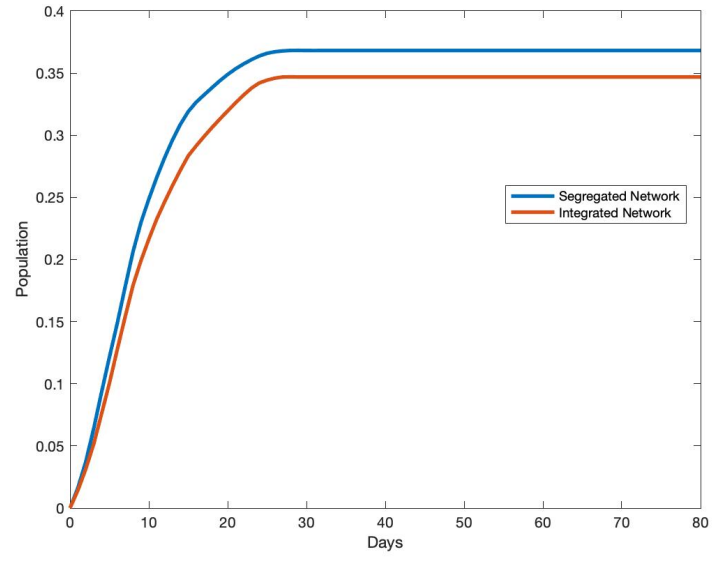

(b) Dynamic of Lockdown

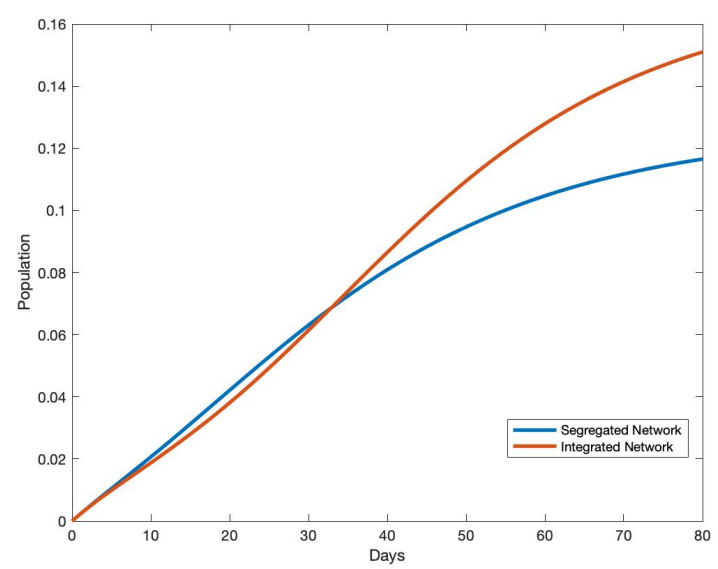

(d) Dynamic of Death 
Figure 16: Dynamics of Outcomes under the Optimal Lockdown Policy: Majority Group (6 to 15)

Patient Zero: Individual 2

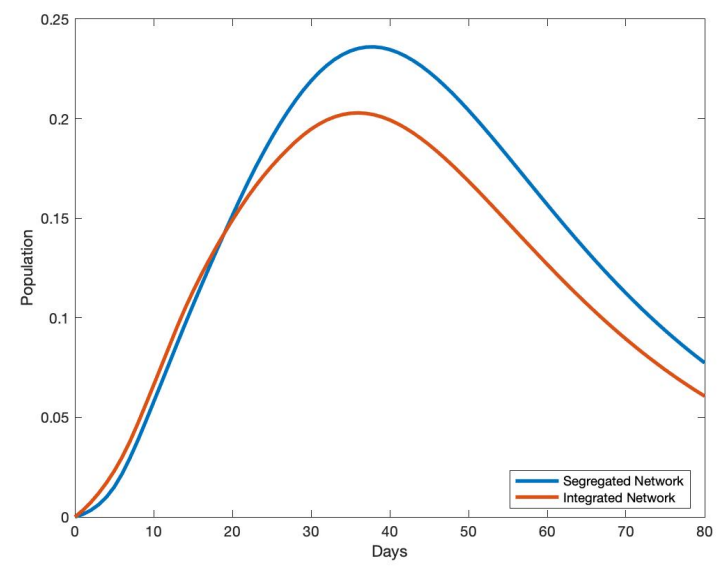

(a) Dynamic of Infection

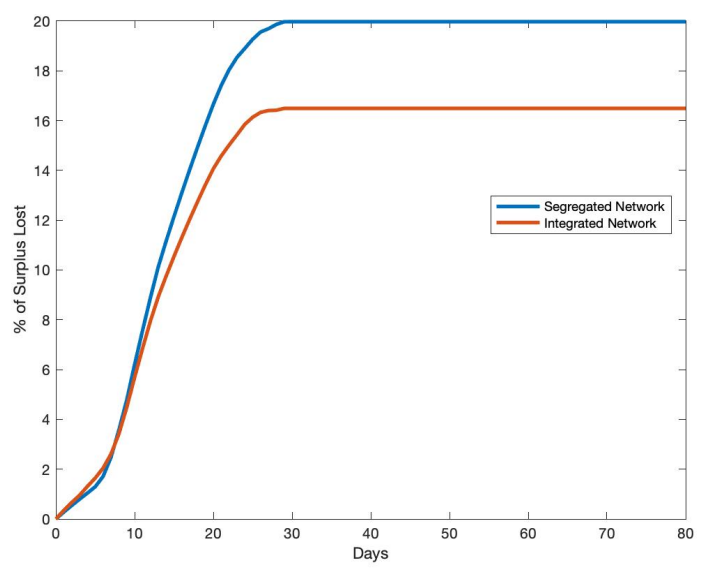

(c) Dynamic of Economic Cost

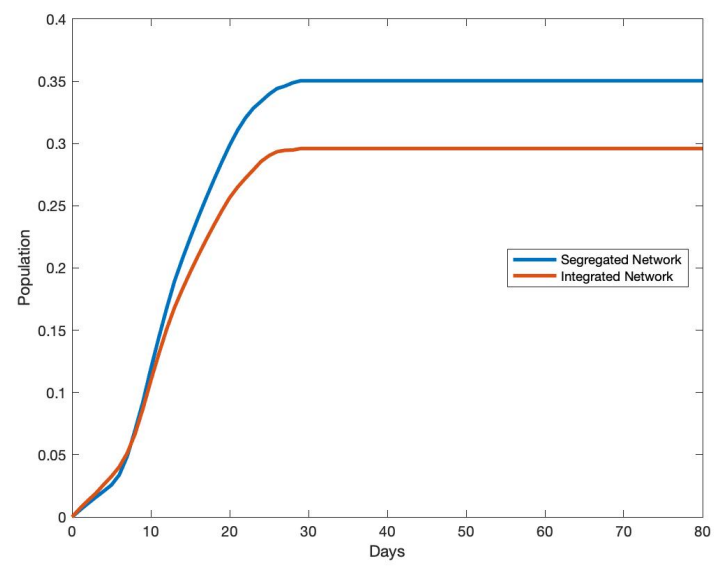

(b) Dynamic of Lockdown

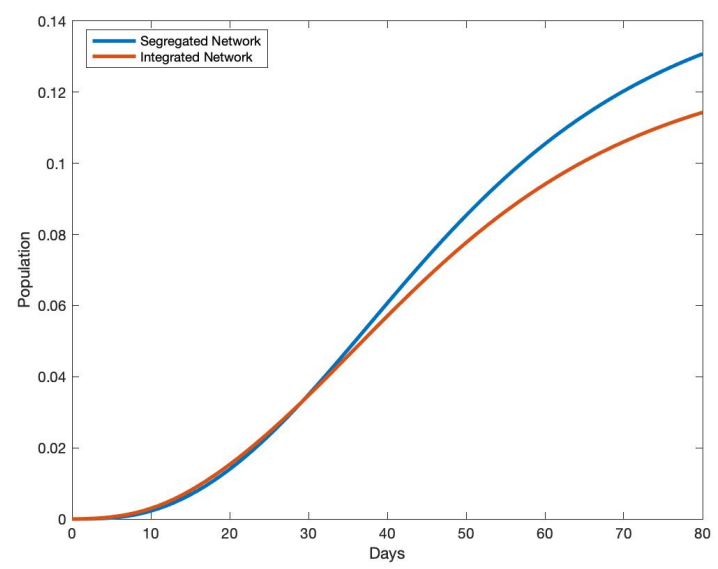

(d) Dynamic of Death 
Figure 17: Effect of Segregation on Minority-Majority Differences in Outcomes: Diff-in-Diffs Analysis

Patient Zero: Individual 2

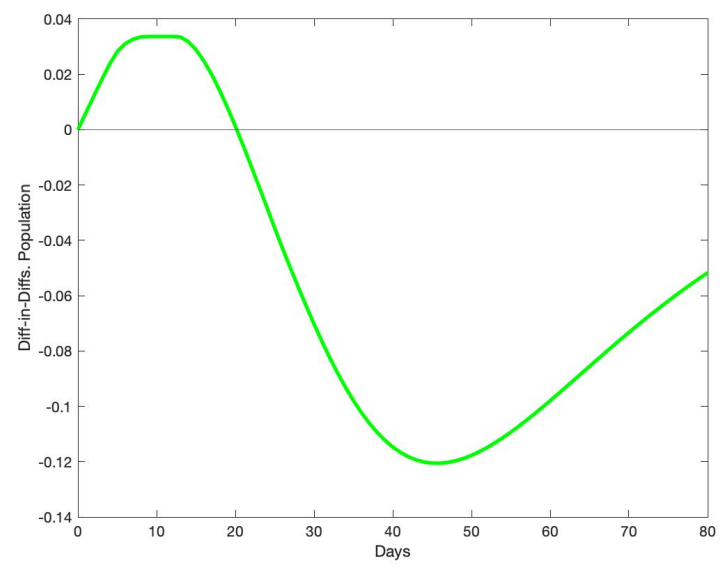

(a) Infection

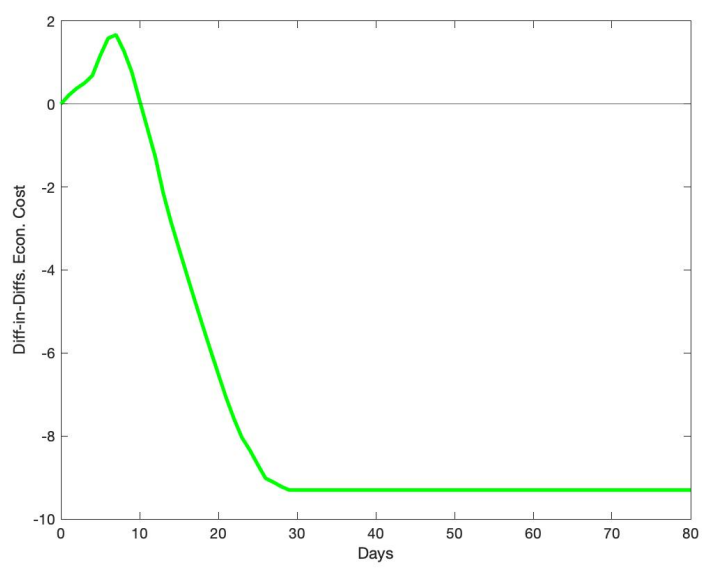

(c) Economic Cost

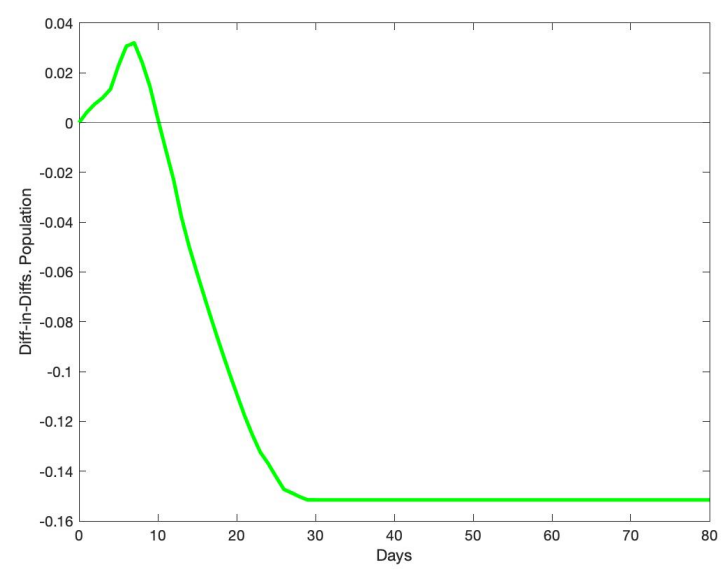

(b) Lockdown

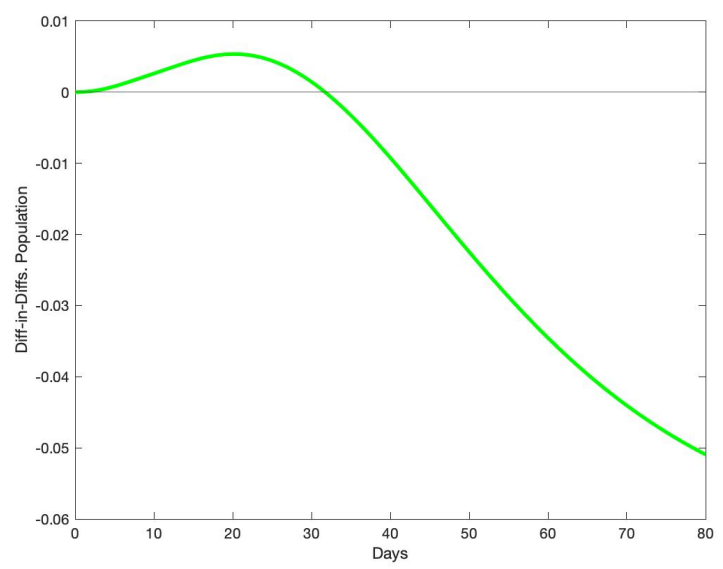

(d) Death 
Figure 18: Dynamics of Outcomes under the Optimal Lockdown Policy: Whole Population Patient Zero: Individual 3

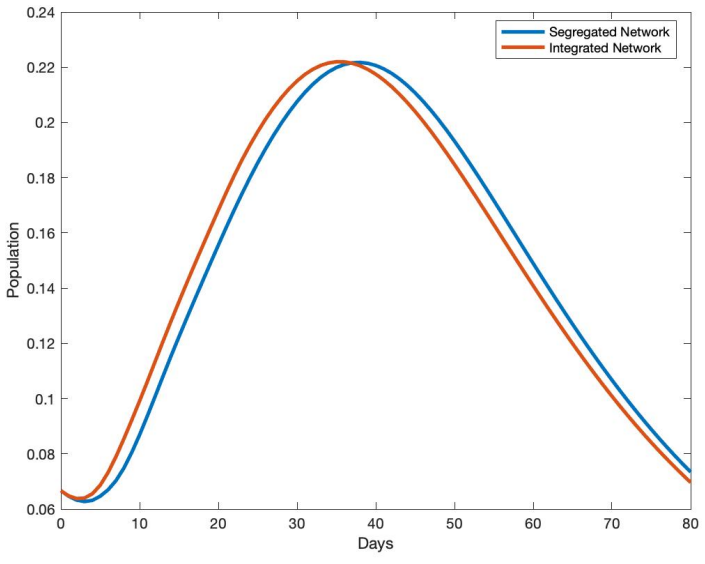

(a) Dynamic of Infection

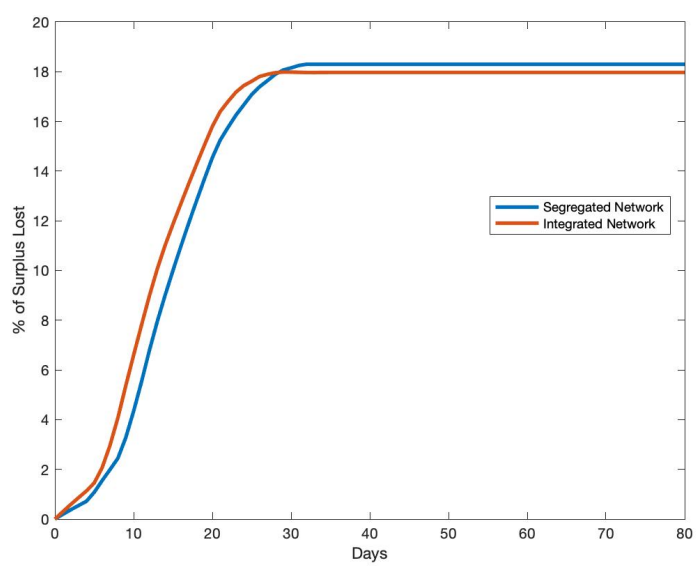

(c) Dynamic of Economic Cost

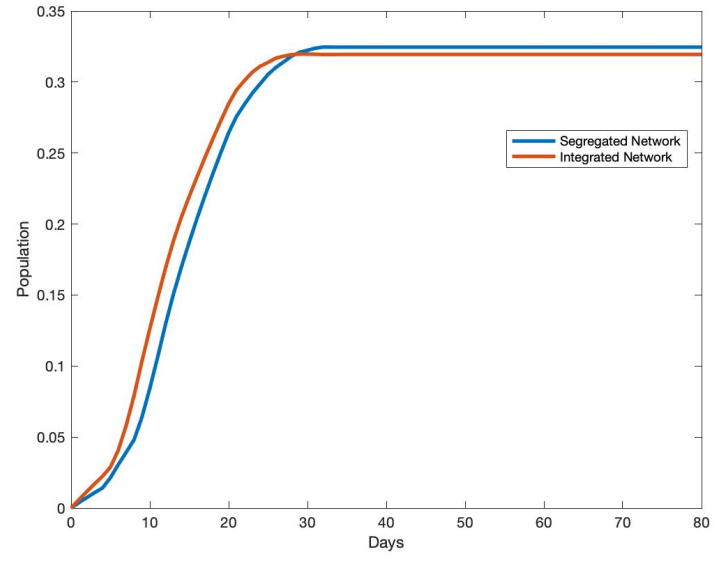

(b) Dynamic of Lockdown

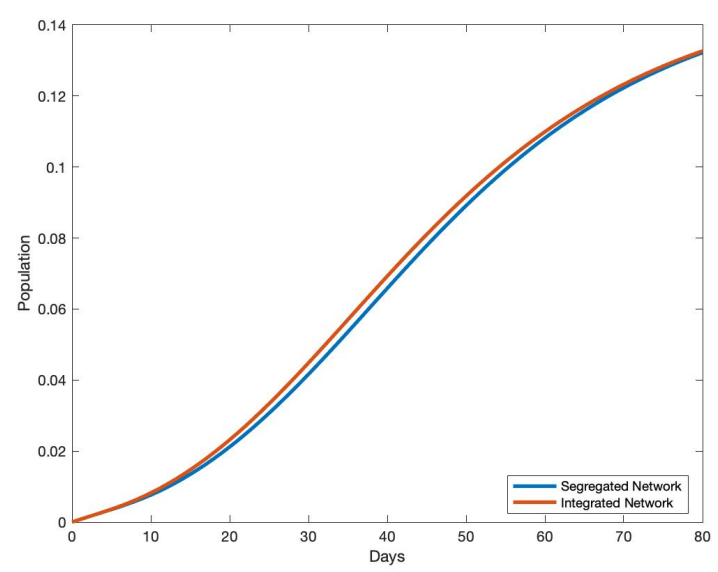

(d) Dynamic of Death 
Figure 19: Dynamics of Outcomes under the Optimal Lockdown Policy: Minority Group (1 to 5) Patient Zero: Individual 3

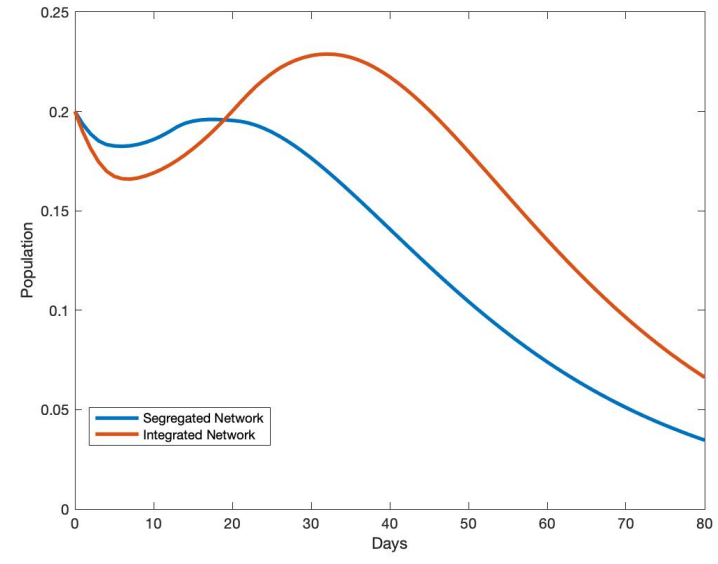

(a) Dynamic of Infection

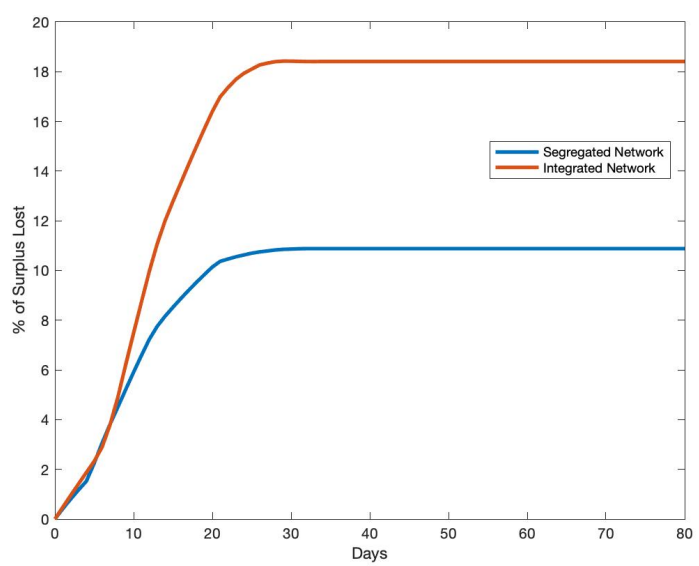

(c) Dynamic of Economic Cost

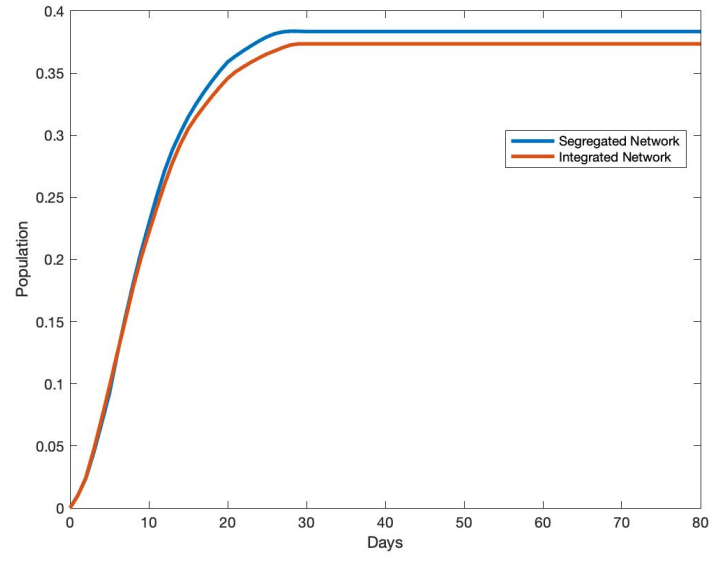

(b) Dynamic of Lockdown

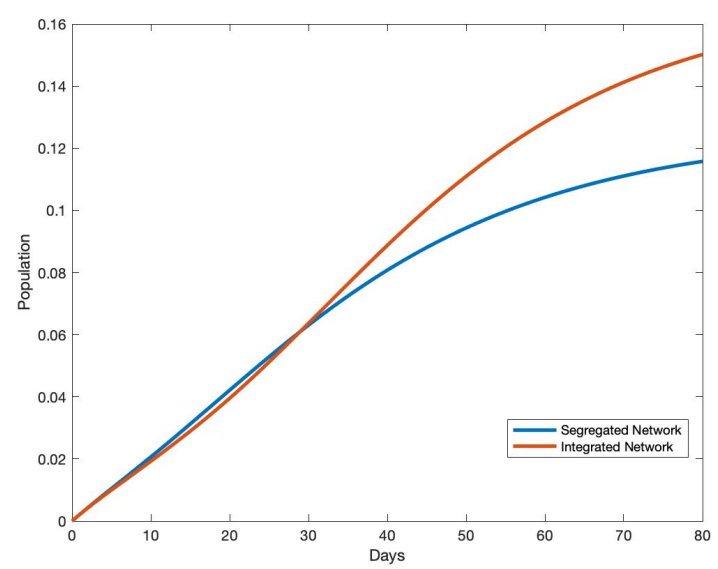

(d) Dynamic of Death 
Figure 20: Dynamics of Outcomes under the Optimal Lockdown Policy: Majority Group (6 to 15) Patient Zero: Individual 3

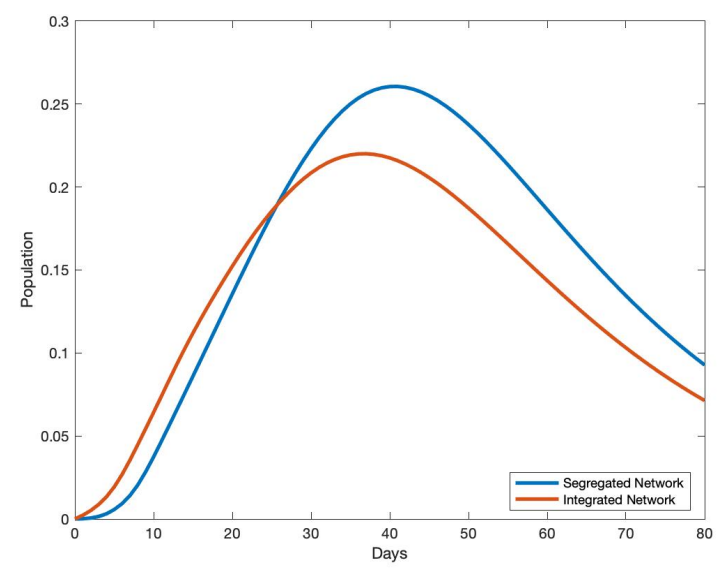

(a) Dynamic of Infection

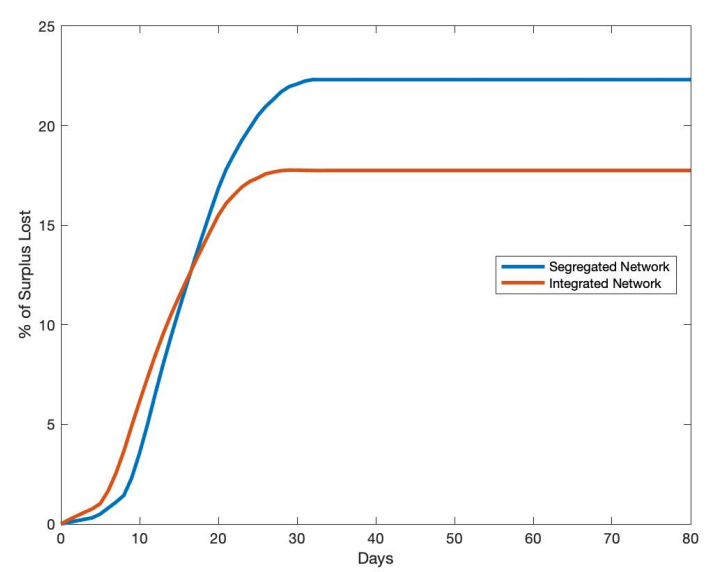

(c) Dynamic of Economic Cost

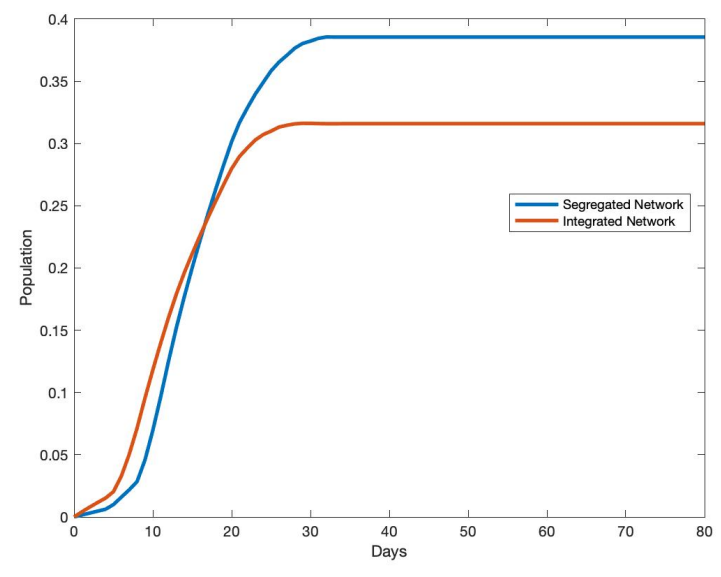

(b) Dynamic of Lockdown

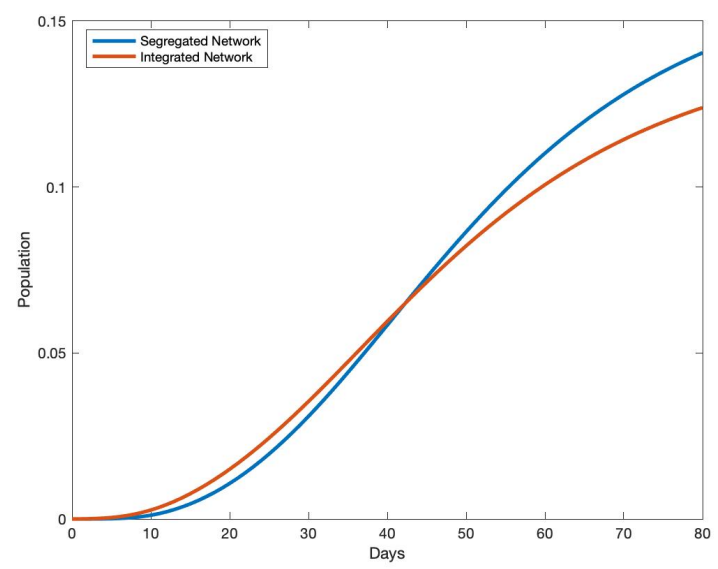

(d) Dynamic of Death 
Figure 21: Effect of Segregation on Minority-Majority Differences in Outcomes: Diff-in-Diffs Analysis

Patient Zero: Individual 3

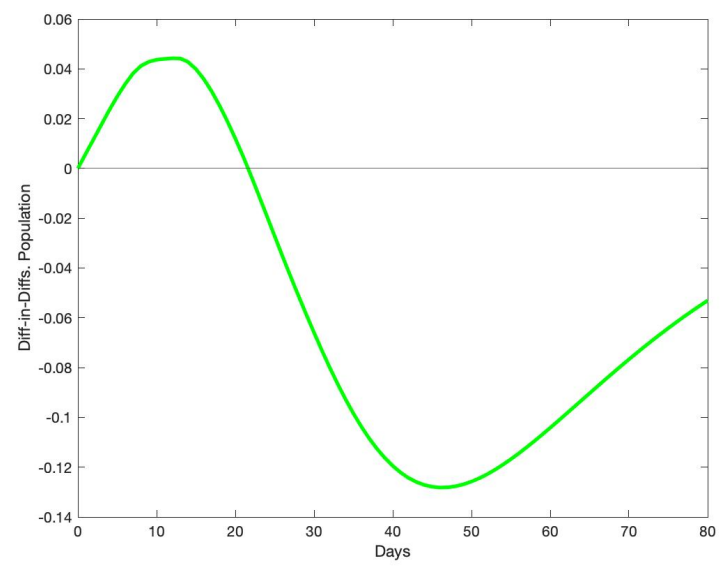

(a) Infection

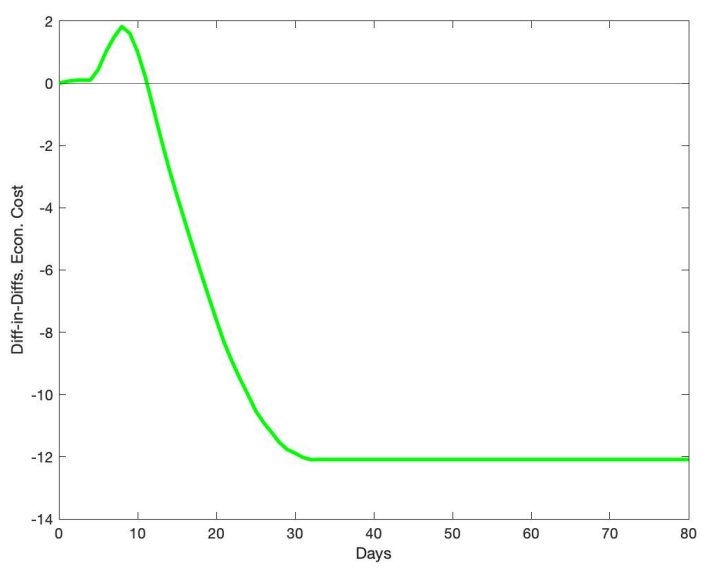

(c) Economic Cost

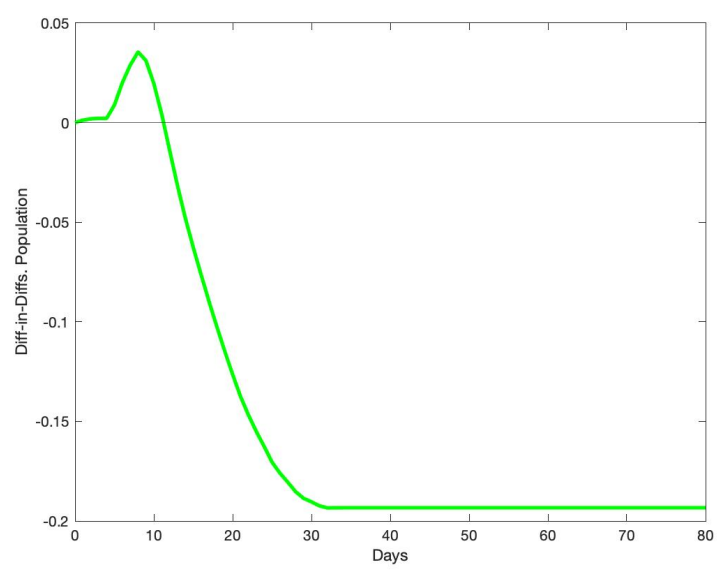

(b) Lockdown

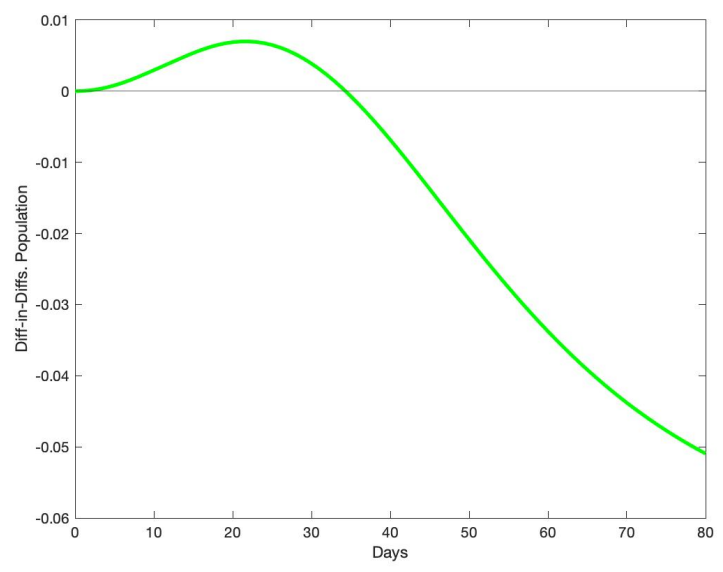

(d) Death 
Figure 22: Dynamics of Outcomes under the Optimal Lockdown Policy: Whole Population Patient Zero: Individual 4

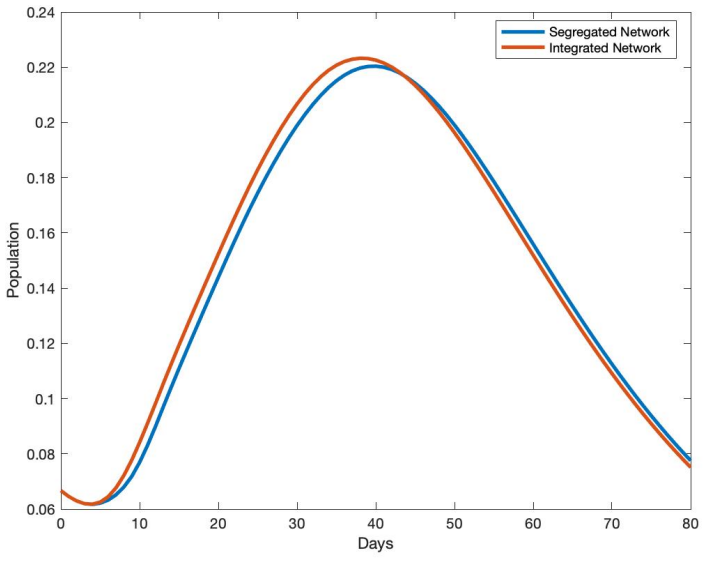

(a) Dynamic of Infection

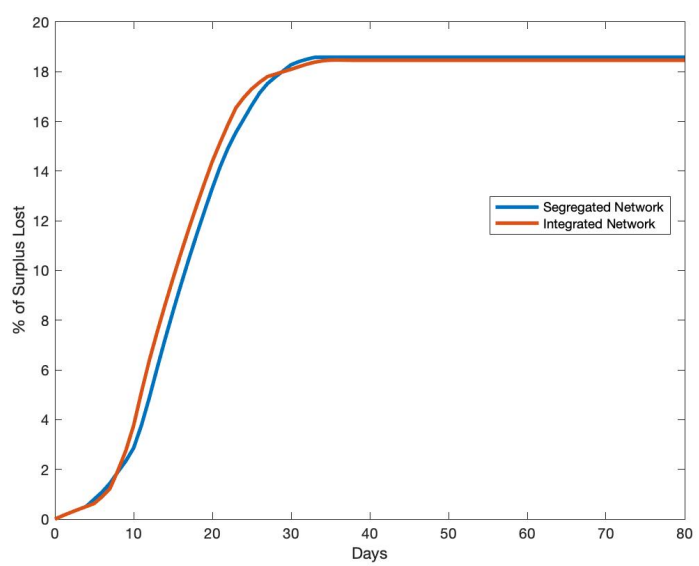

(c) Dynamic of Economic Cost

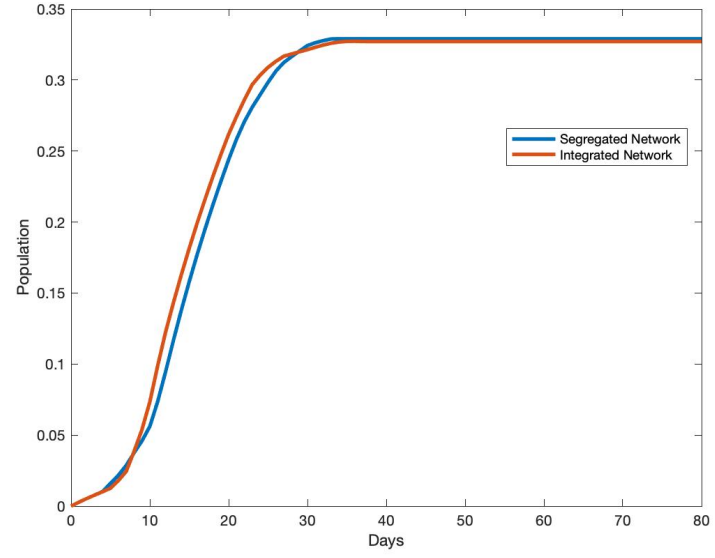

(b) Dynamic of Lockdown

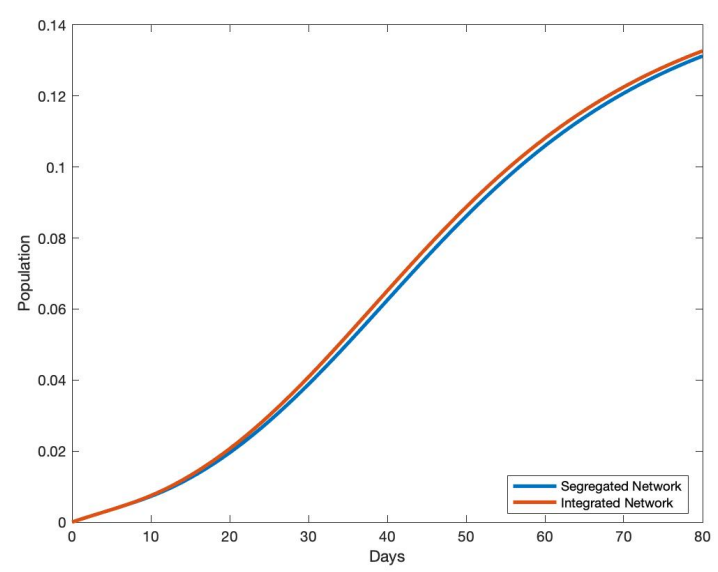

(d) Dynamic of Death 
Figure 23: Dynamics of Outcomes under the Optimal Lockdown Policy: Minority Group (1 to 5) Patient Zero: Individual 4

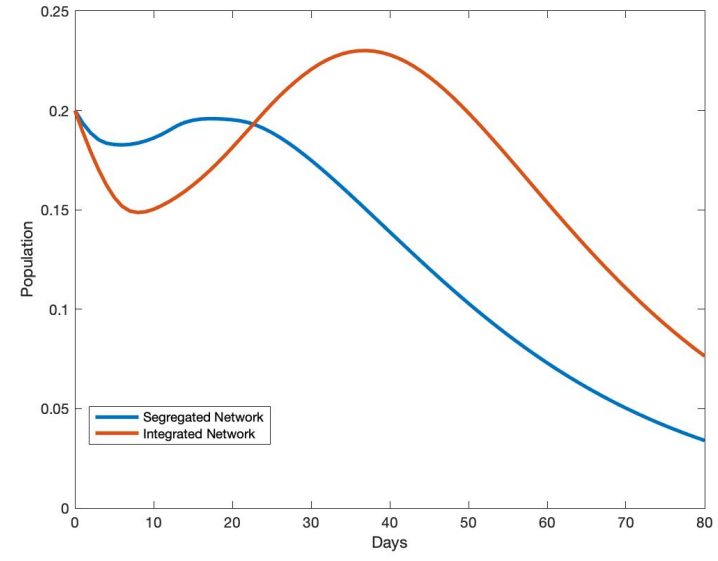

(a) Dynamic of Infection

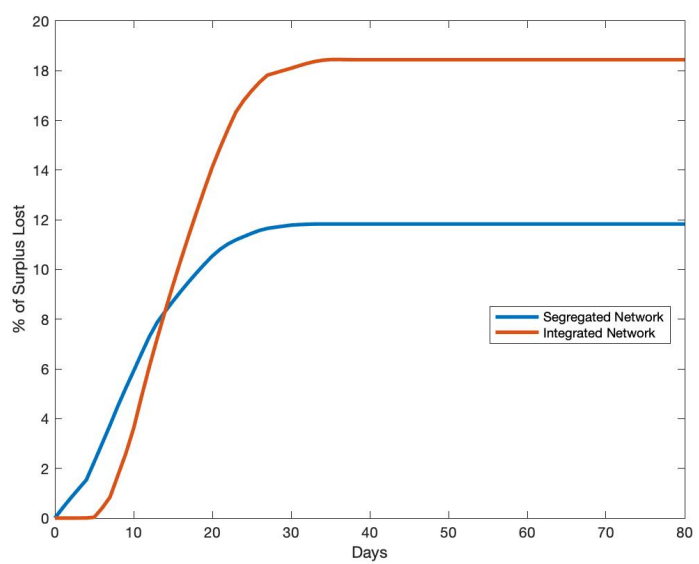

(c) Dynamic of Economic Cost

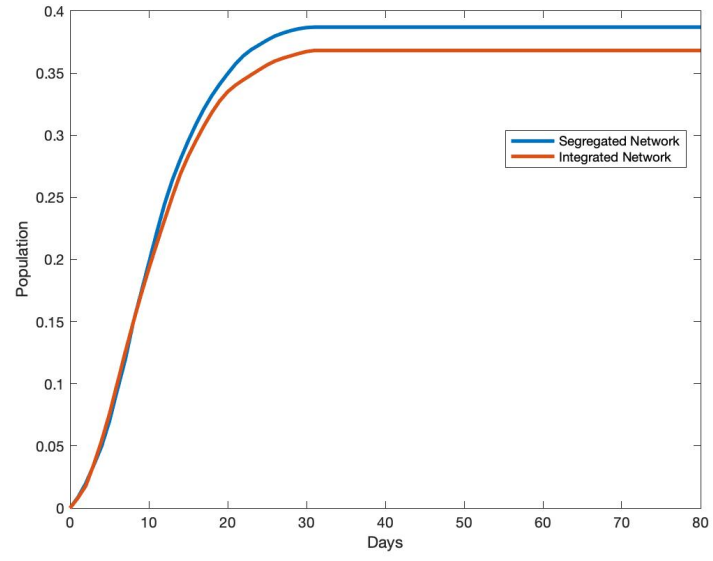

(b) Dynamic of Lockdown

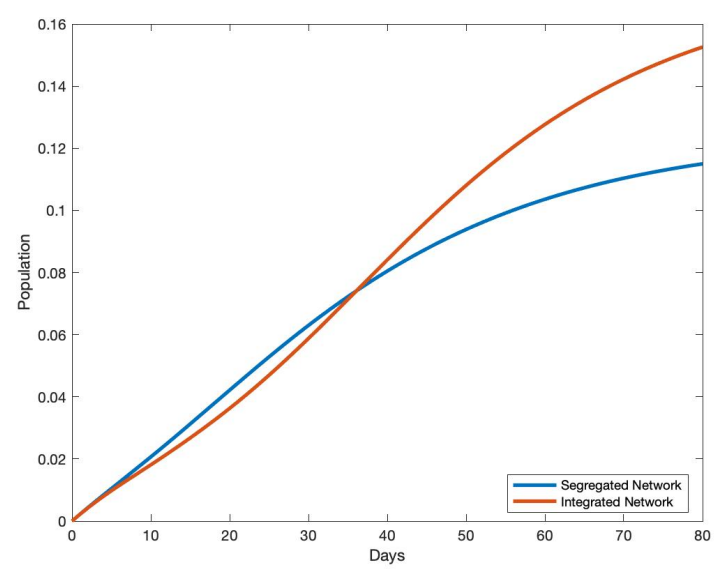

(d) Dynamic of Death 
Figure 24: Dynamics of Outcomes under the Optimal Lockdown Policy: Majority Group (6 to 15) Patient Zero: Individual 4

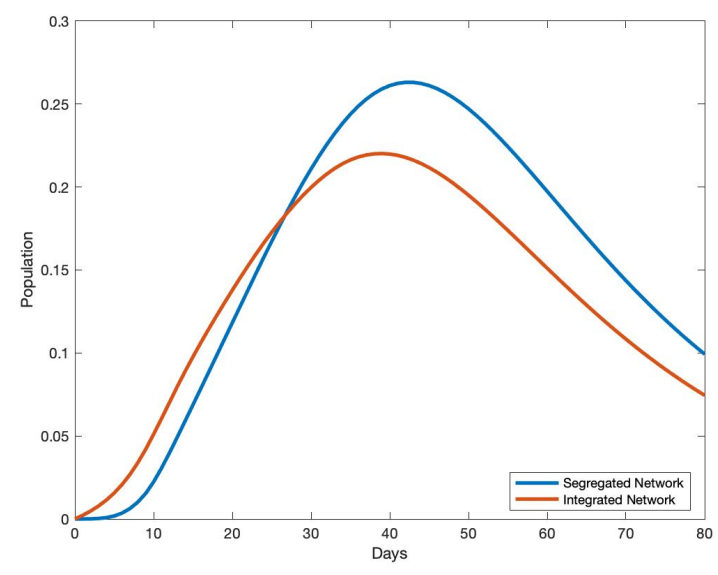

(a) Dynamic of Infection

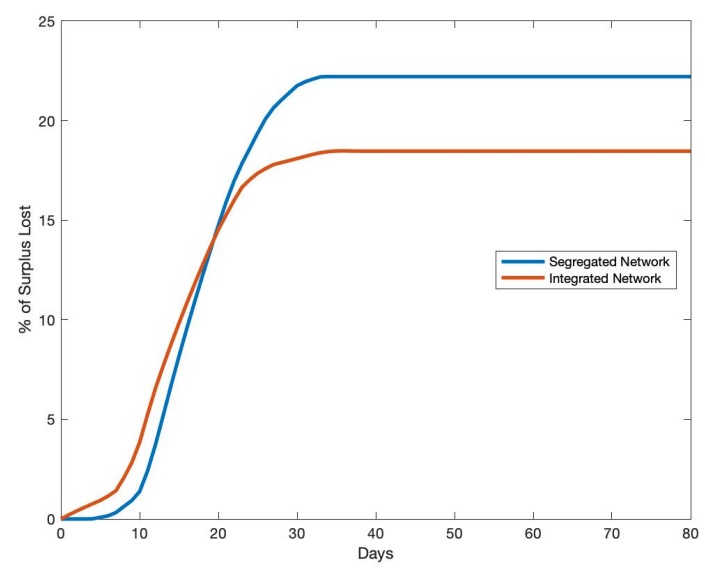

(c) Dynamic of Economic Cost

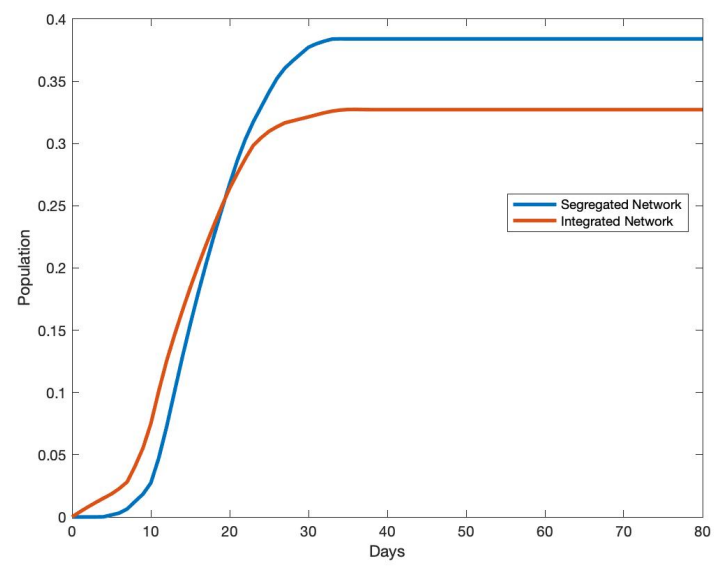

(b) Dynamic of Lockdown

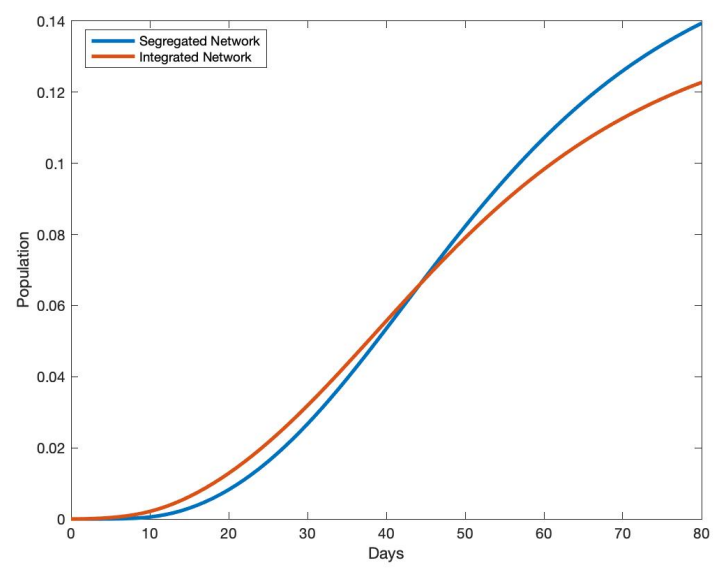

(d) Dynamic of Death 
Figure 25: Effect of Segregation on Minority-Majority Differences in Outcomes: Diff-in-Diffs Analysis

Patient Zero: Individual 4

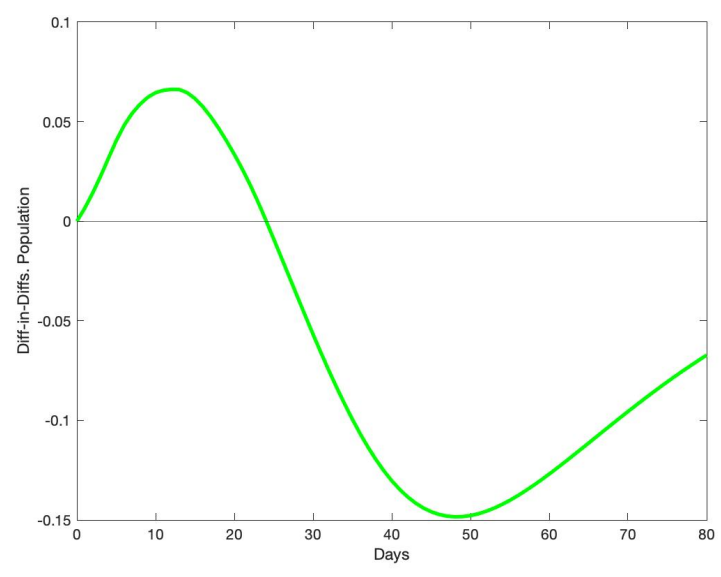

(a) Infection

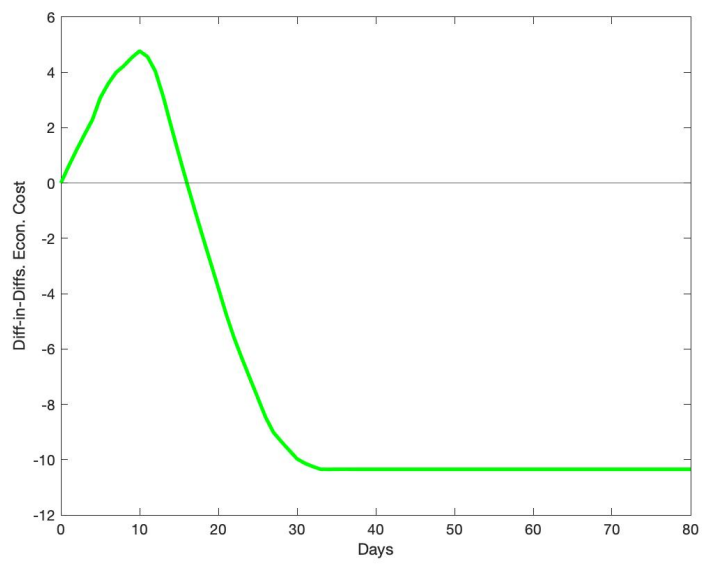

(c) Economic Cost

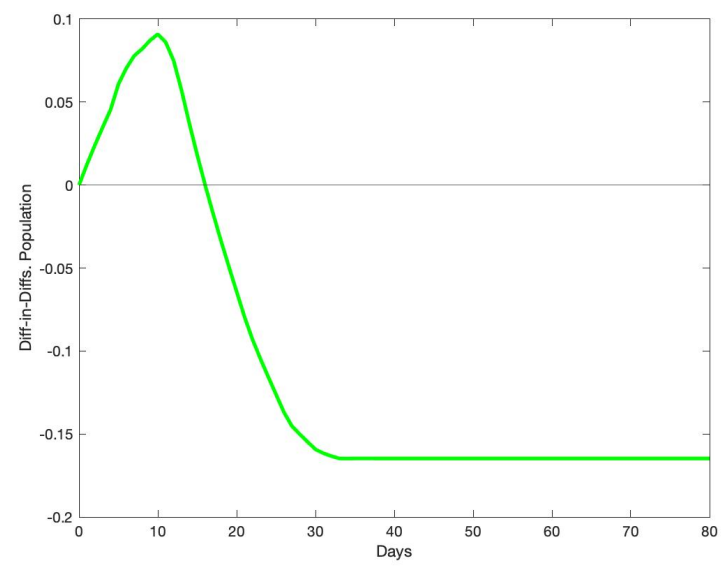

(b) Lockdown

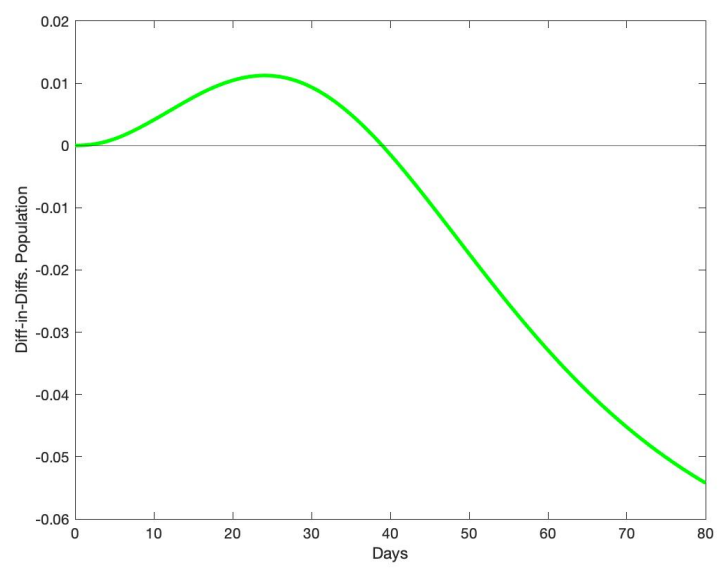

(d) Death 
Figure 26: Dynamics of Outcomes under the Optimal Lockdown Policy: Whole Population Patient Zero: Individual 5

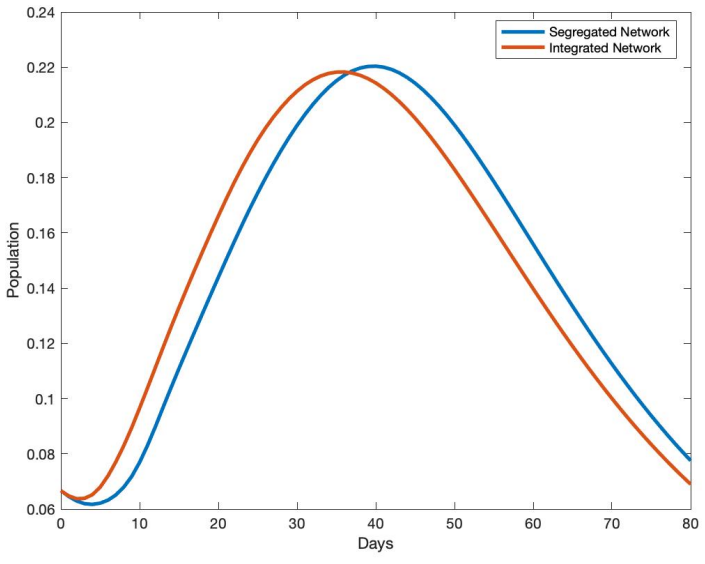

(a) Dynamic of Infection

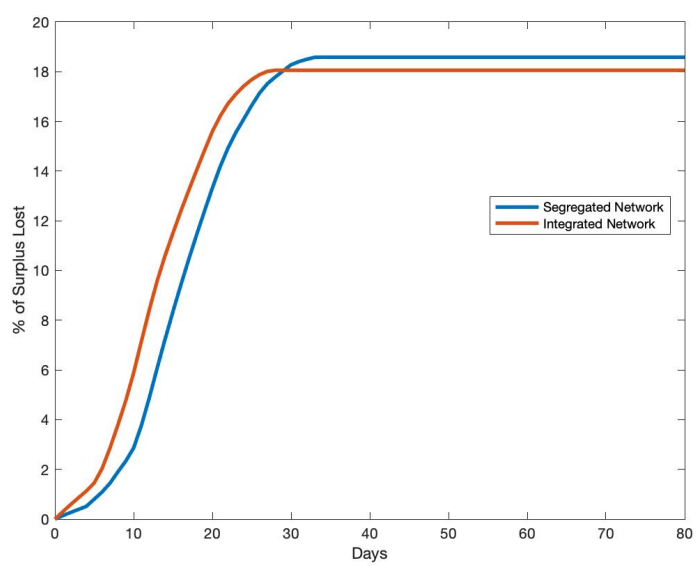

(c) Dynamic of Economic Cost

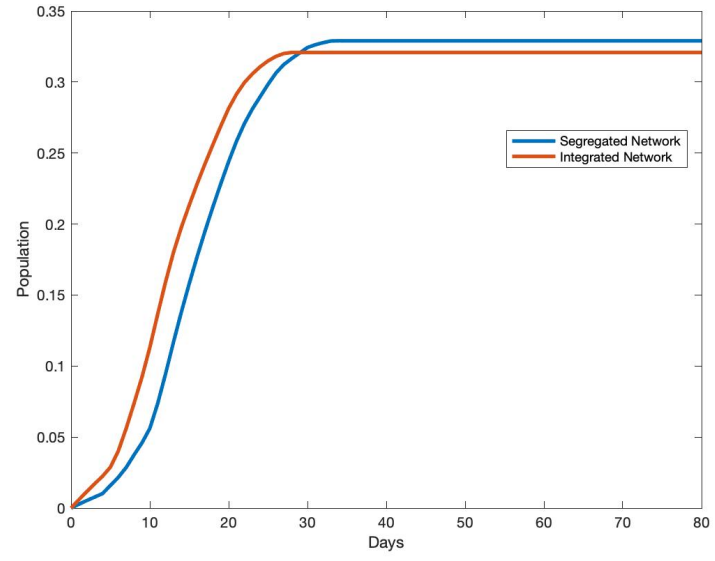

(b) Dynamic of Lockdown

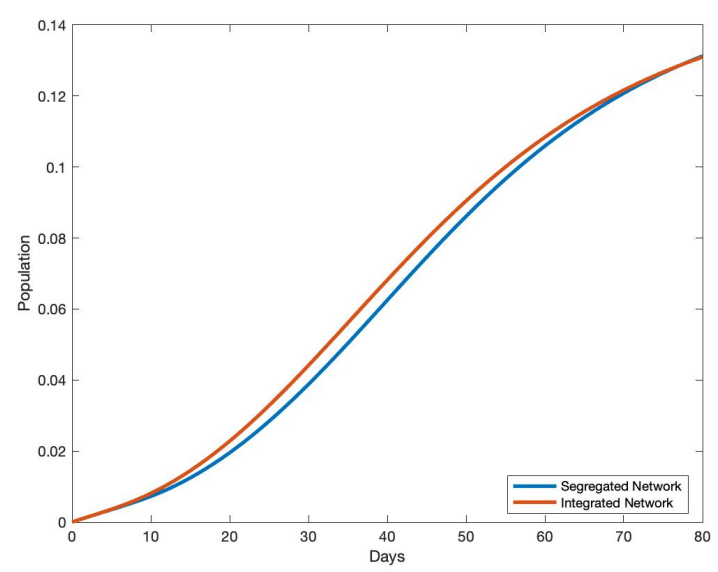

(d) Dynamic of Death 
Figure 27: Dynamics of Outcomes under the Optimal Lockdown Policy: Minority Group (1 to 5) Patient Zero: Individual 5

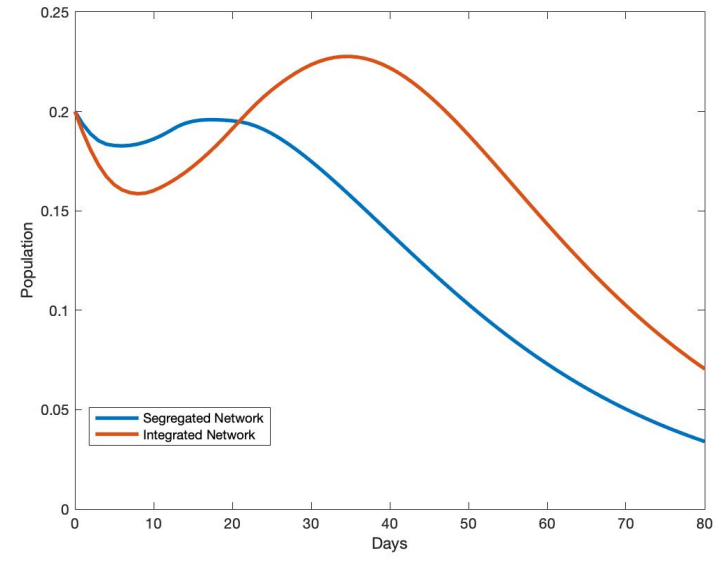

(a) Dynamic of Infection

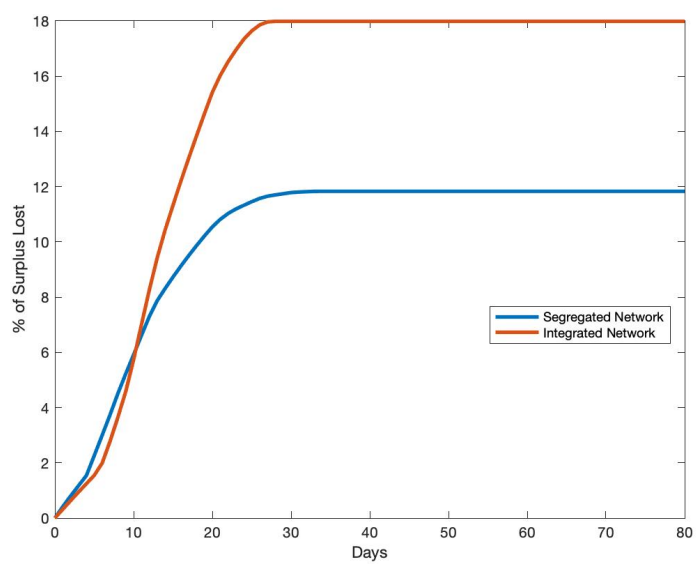

(c) Dynamic of Economic Cost

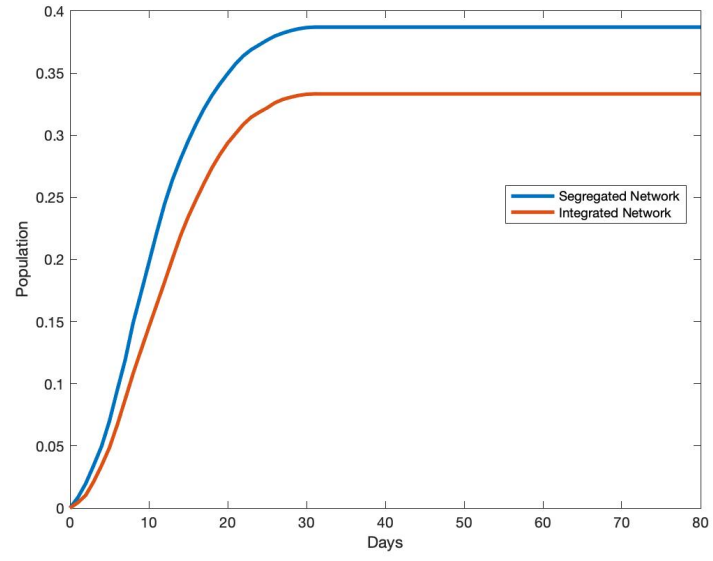

(b) Dynamic of Lockdown

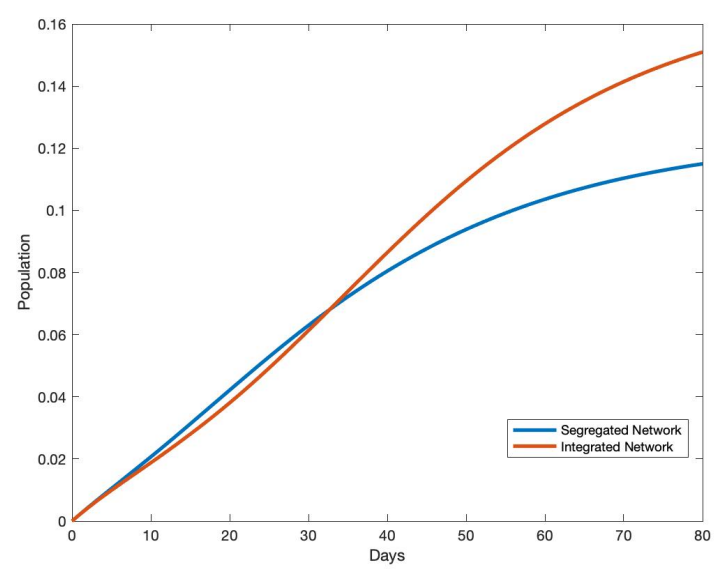

(d) Dynamic of Death 
Figure 28: Dynamics of Outcomes under the Optimal Lockdown Policy: Majority Group (6 to 15) Patient Zero: Individual 5

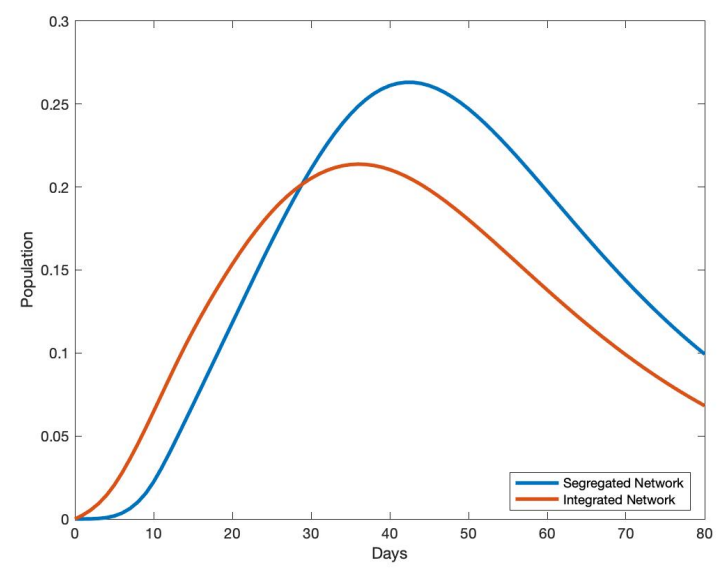

(a) Dynamic of Infection

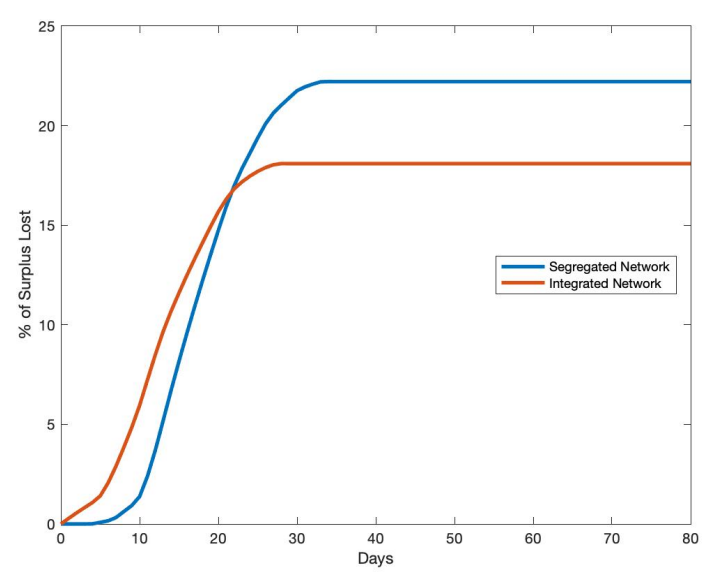

(c) Dynamic of Economic Cost

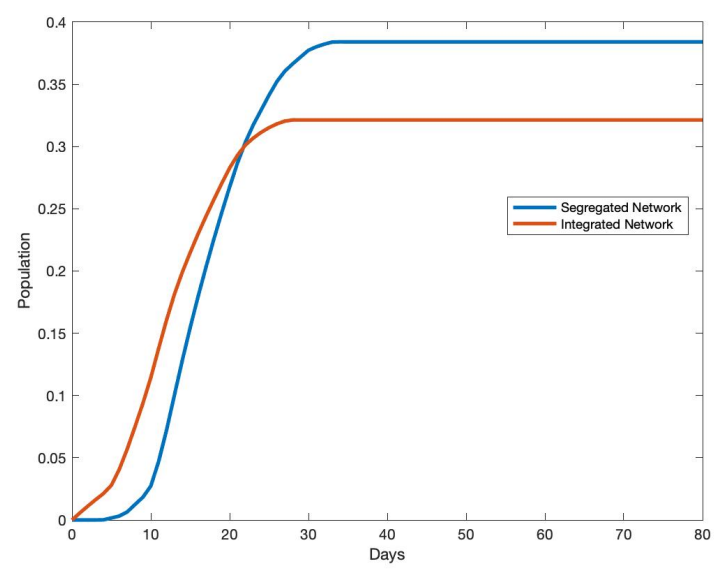

(b) Dynamic of Lockdown

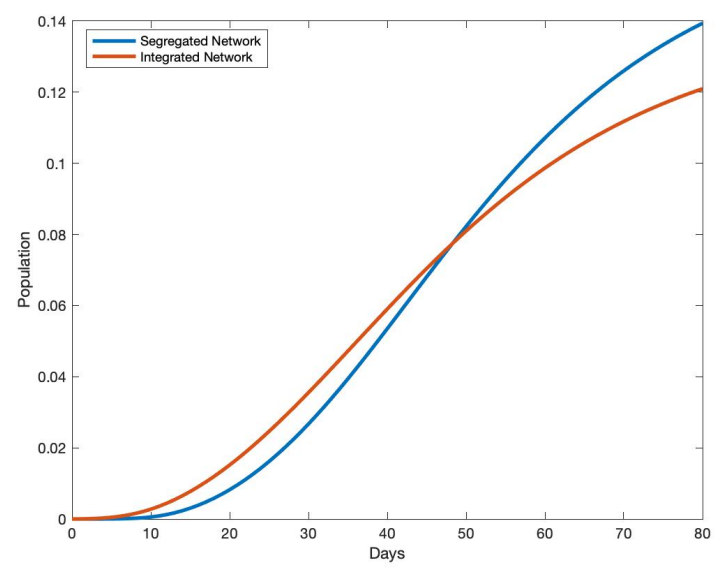

(d) Dynamic of Death 
Figure 29: Effect of Segregation on Minority-Majority Differences in Outcomes: Diff-in-Diffs Analysis

Patient Zero: Individual 5

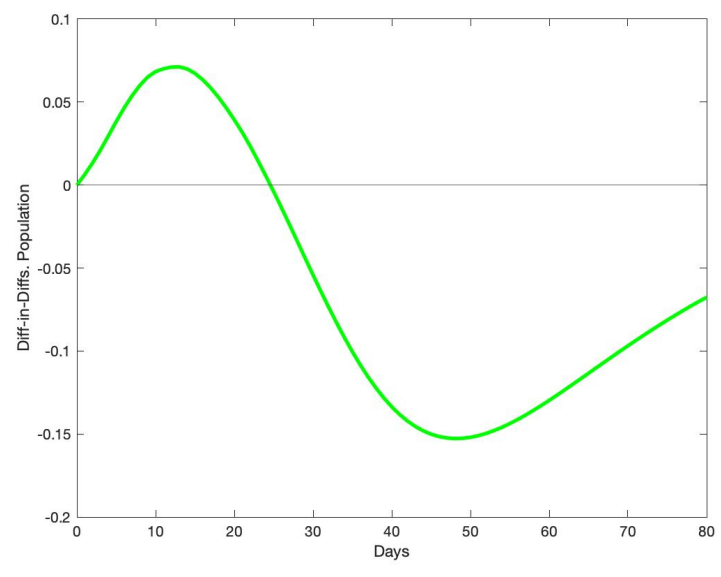

(a) Infection

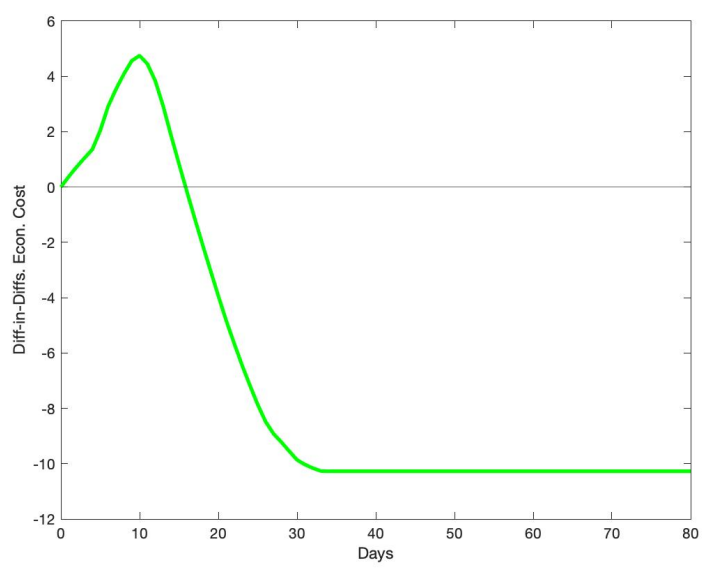

(c) Economic Cost

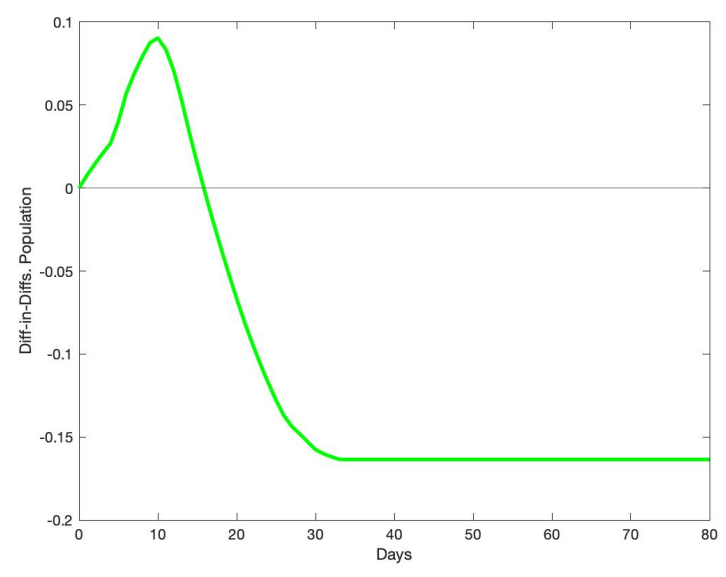

(b) Lockdown

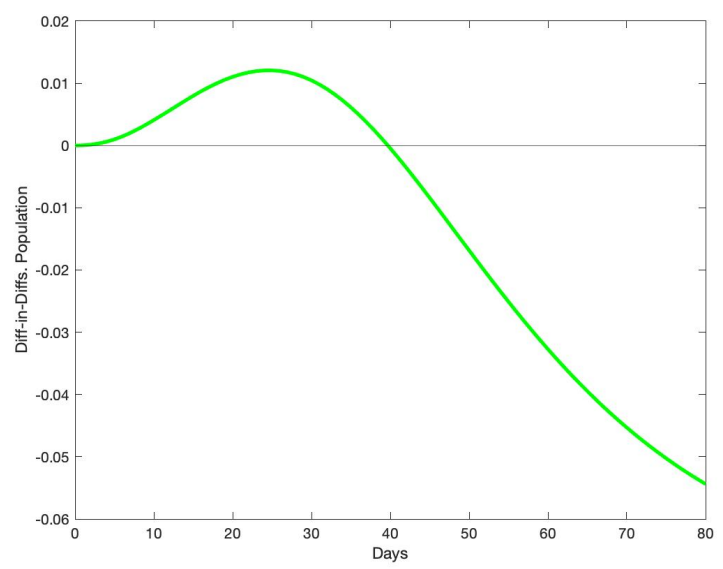

(d) Death 
Figure 30: Dynamics of Outcomes under the Optimal Lockdown Policy: Whole Population Patient Zero: Individual 6

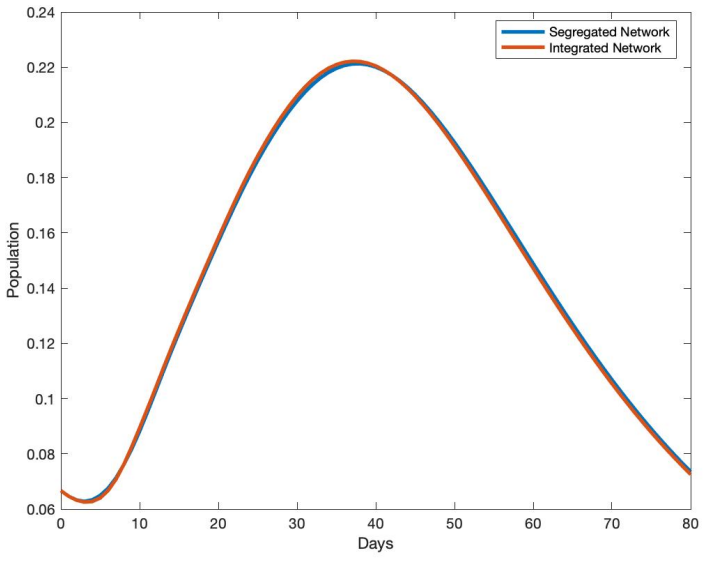

(a) Dynamic of Infection

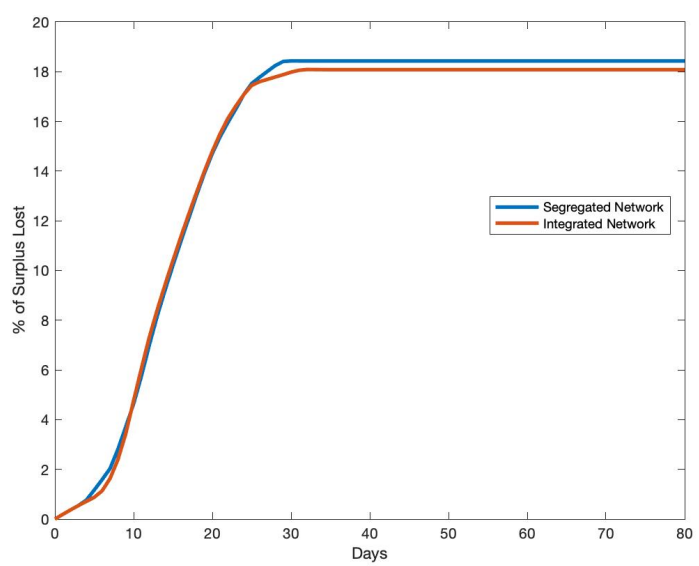

(c) Dynamic of Economic Cost

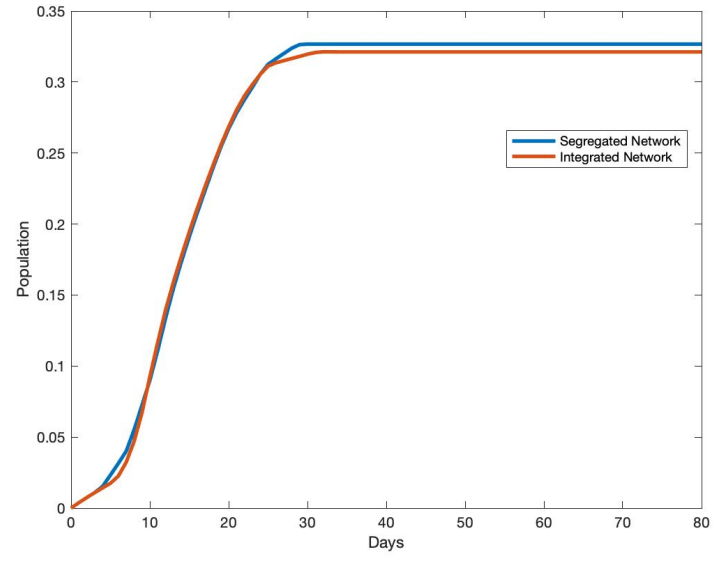

(b) Dynamic of Lockdown

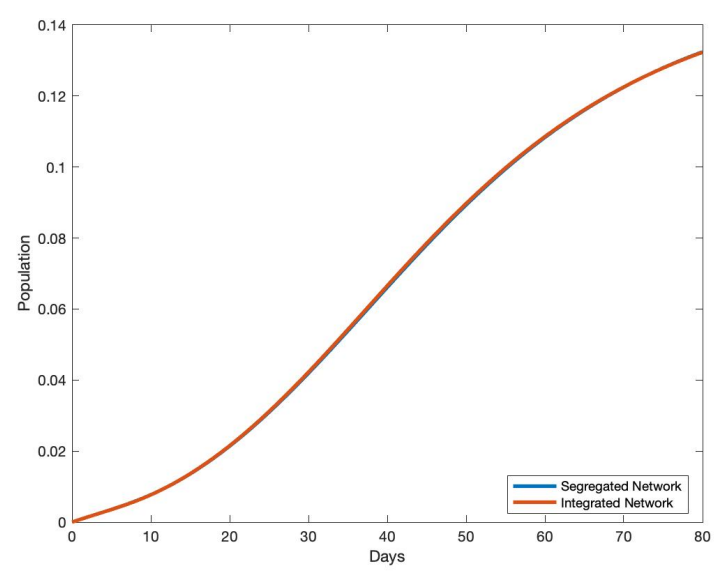

(d) Dynamic of Death 
Figure 31: Dynamics of Outcomes under the Optimal Lockdown Policy: Minority Group (1 to 5) Patient Zero: Individual 6

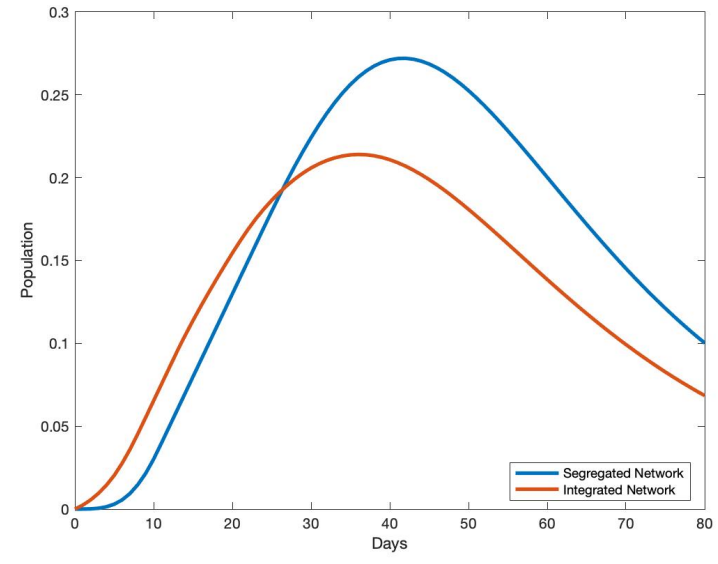

(a) Dynamic of Infection

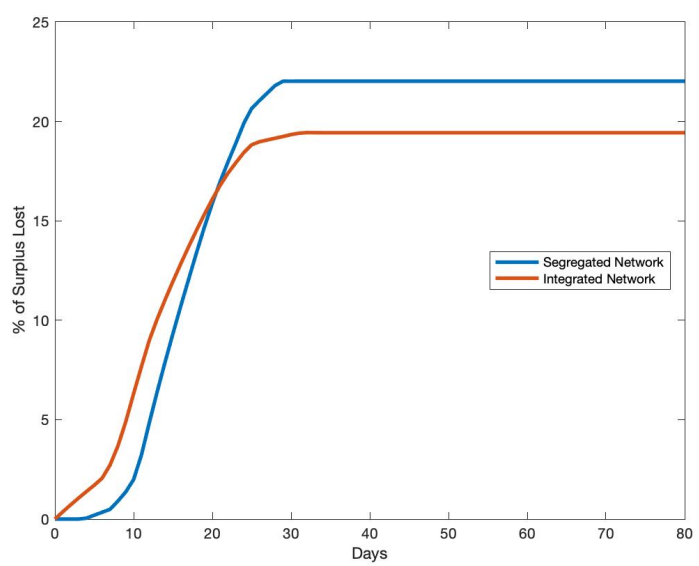

(c) Dynamic of Economic Cost

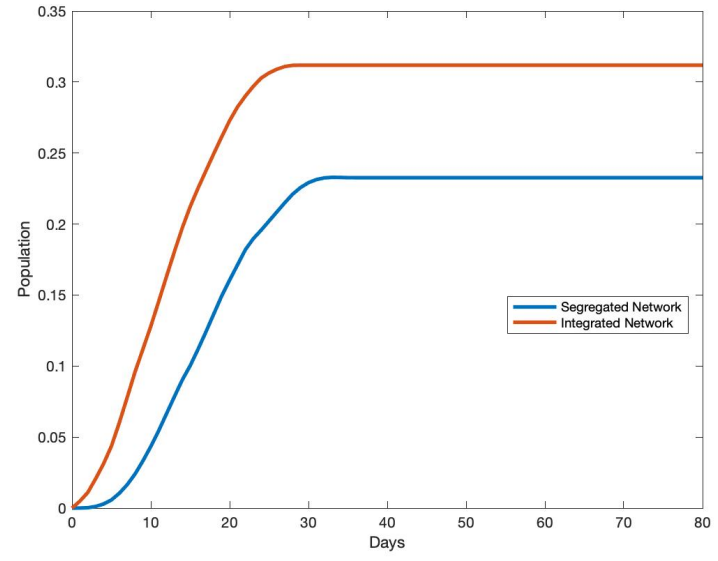

(b) Dynamic of Lockdown

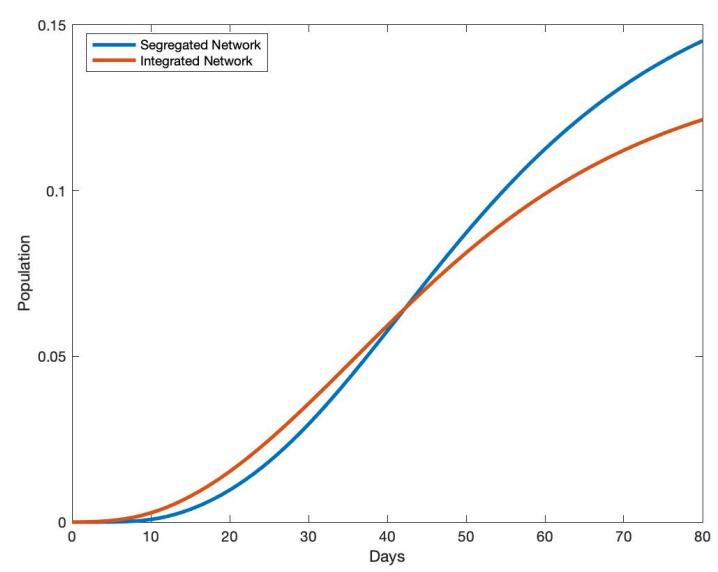

(d) Dynamic of Death 
Figure 32: Dynamics of Outcomes under the Optimal Lockdown Policy: Majority Group (6 to 15) Patient Zero: Individual 6

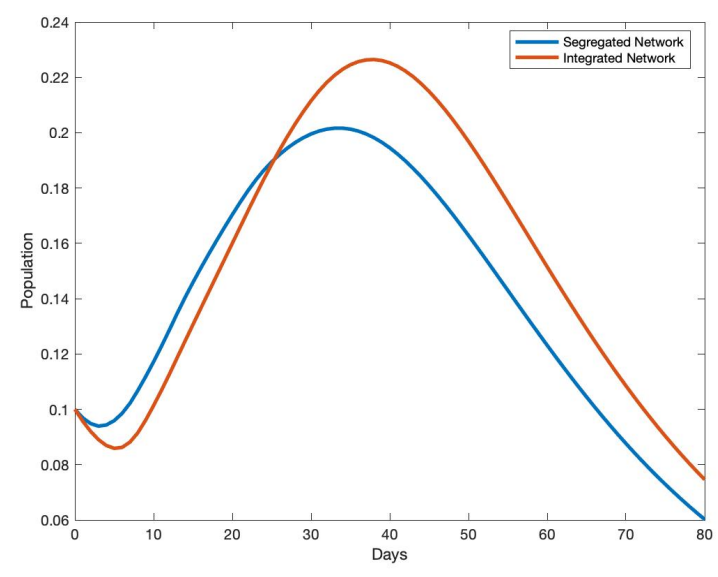

(a) Dynamic of Infection

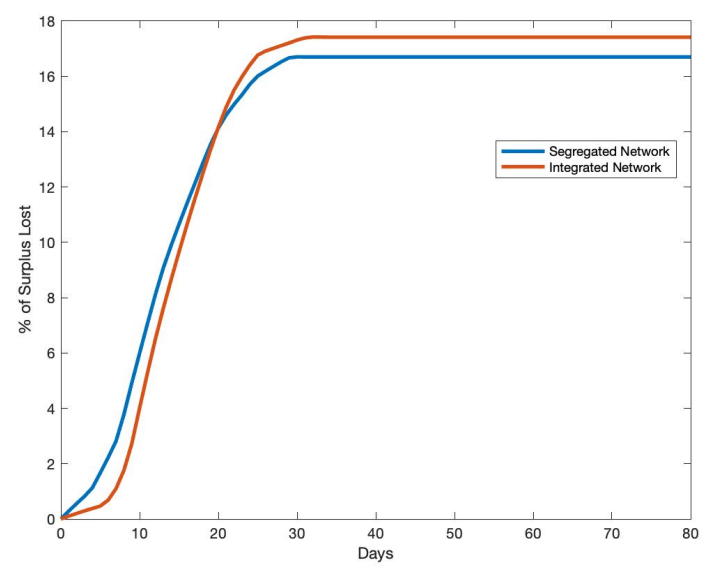

(c) Dynamic of Economic Cost

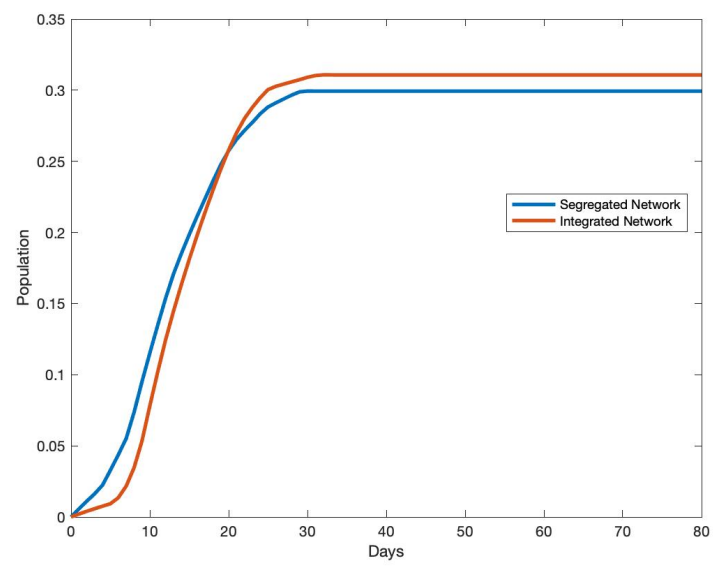

(b) Dynamic of Lockdown

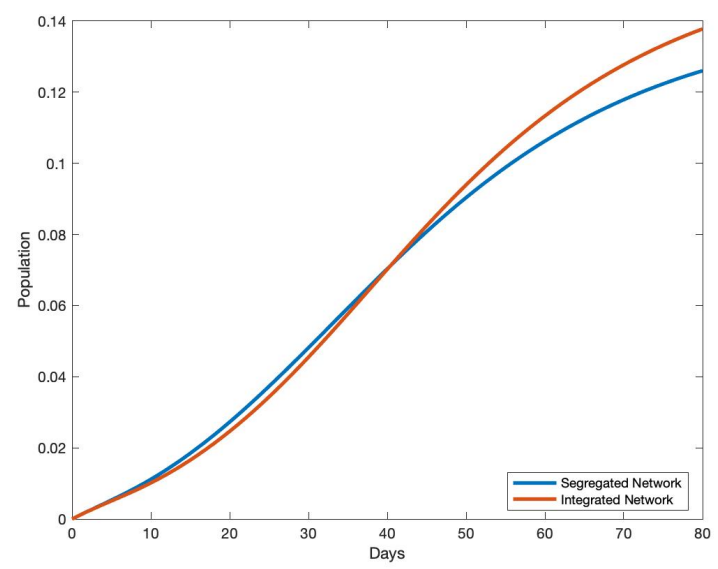

(d) Dynamic of Death 
Figure 33: Effect of Segregation on Minority-Majority Differences in Outcomes: Diff-in-Diffs Analysis

Patient Zero: Individual 6

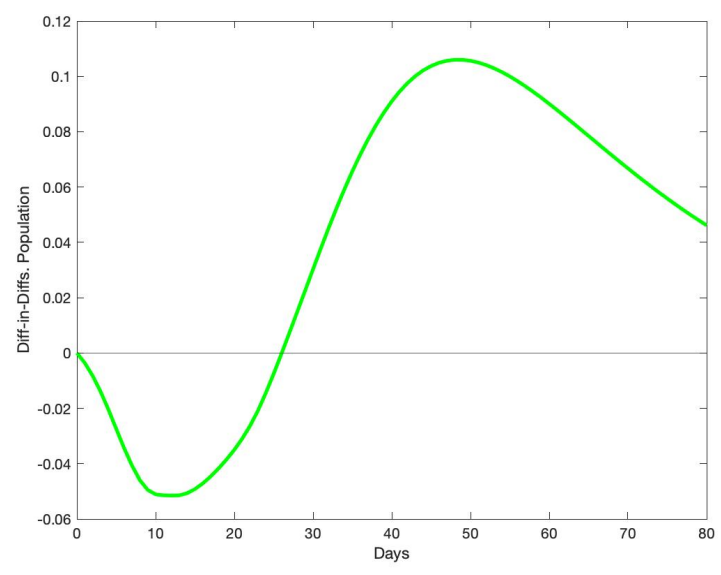

(a) Infection

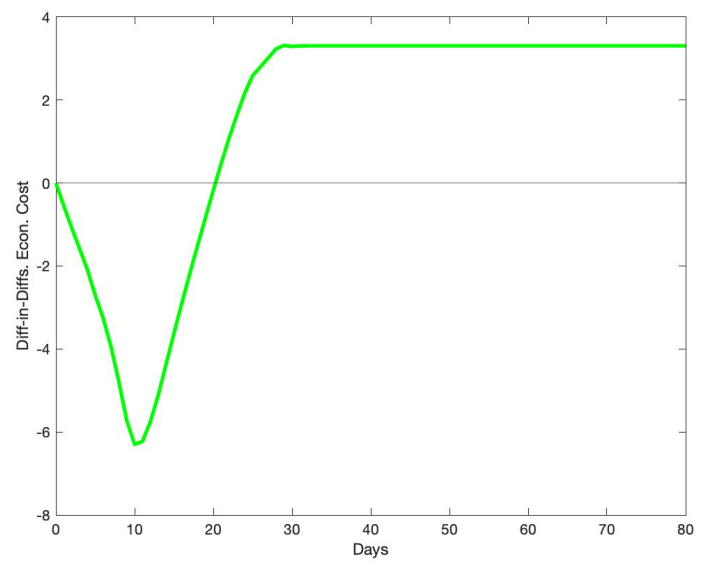

(c) Economic Cost

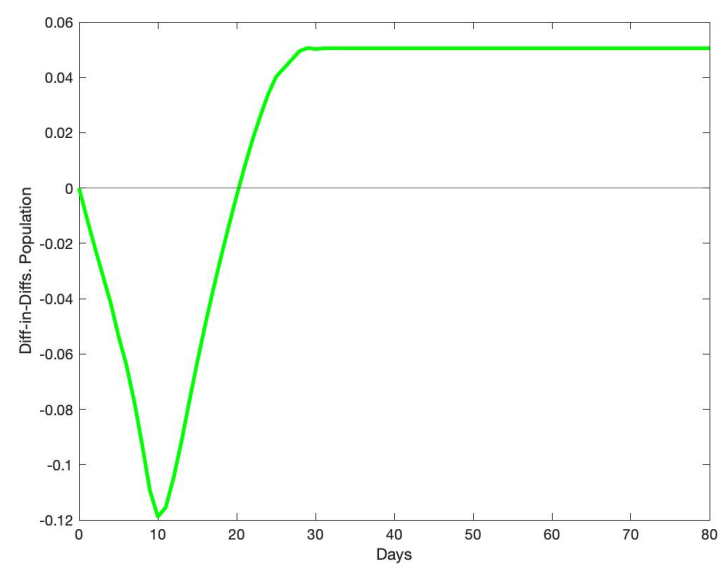

(b) Lockdown

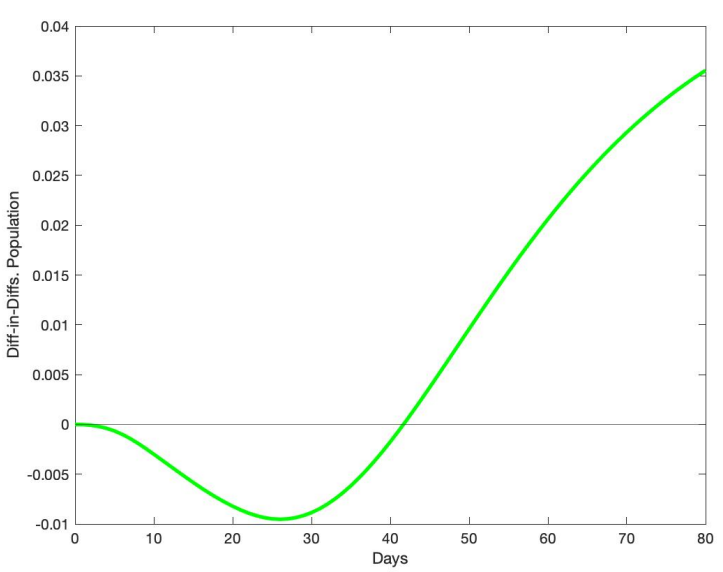

(d) Death 
Figure 34: Dynamics of Outcomes under the Optimal Lockdown Policy: Whole Population Patient Zero: Individual 7

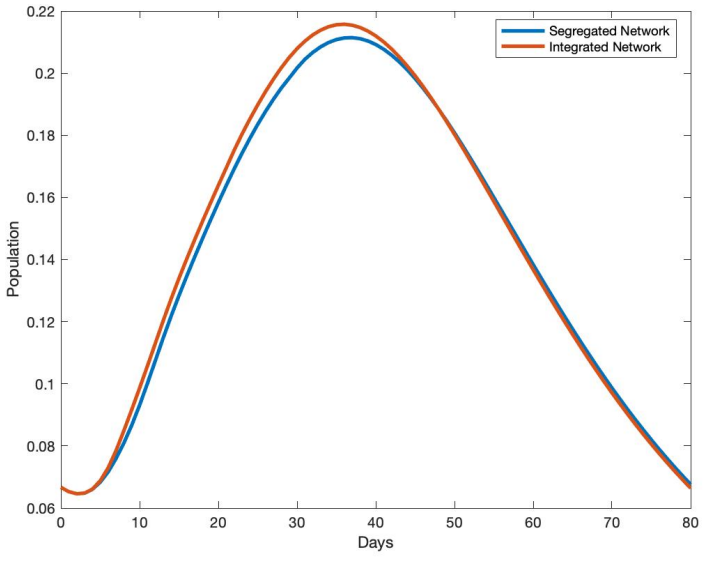

(a) Dynamic of Infection

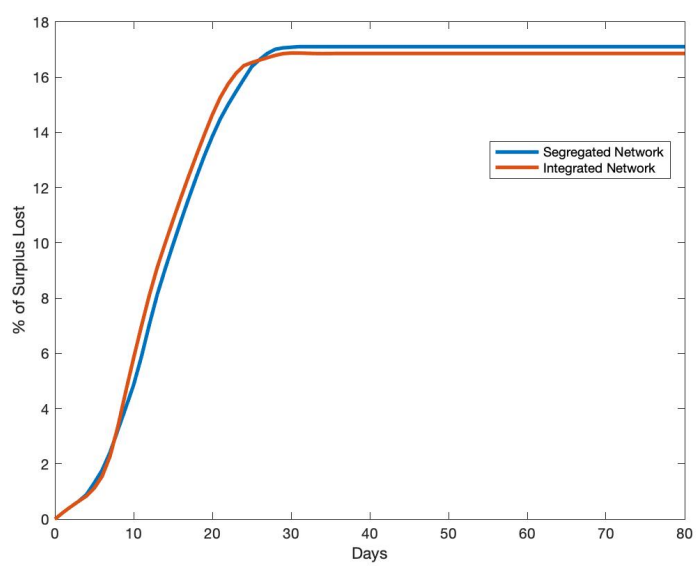

(c) Dynamic of Economic Cost

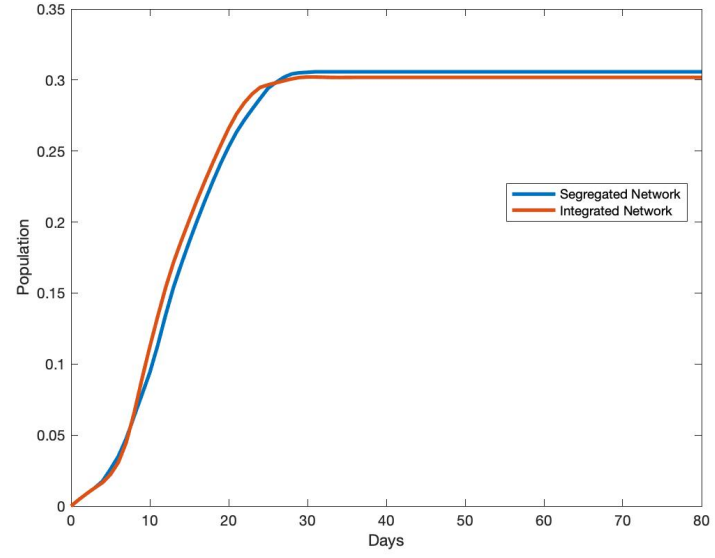

(b) Dynamic of Lockdown

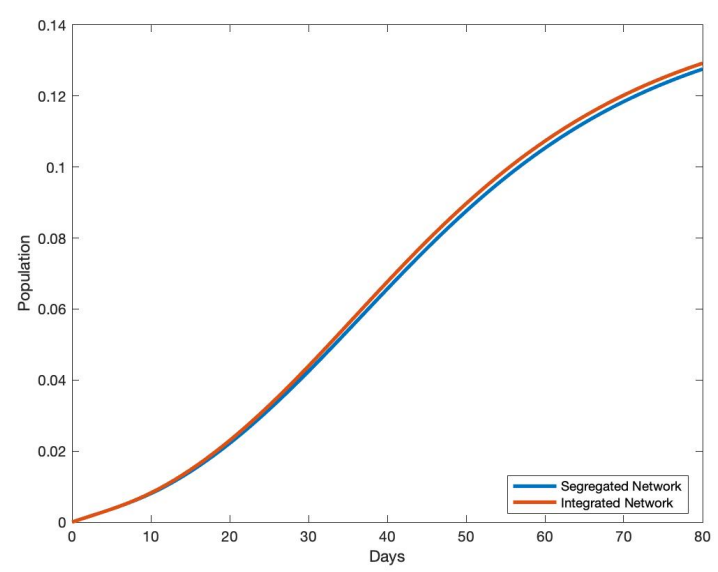

(d) Dynamic of Death 
Figure 35: Dynamics of Outcomes under the Optimal Lockdown Policy: Minority Group (1 to 5) Patient Zero: Individual 7

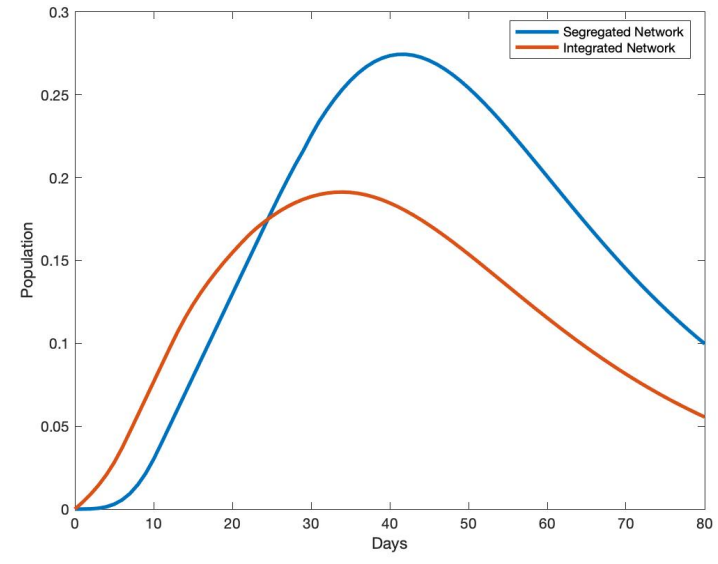

(a) Dynamic of Infection

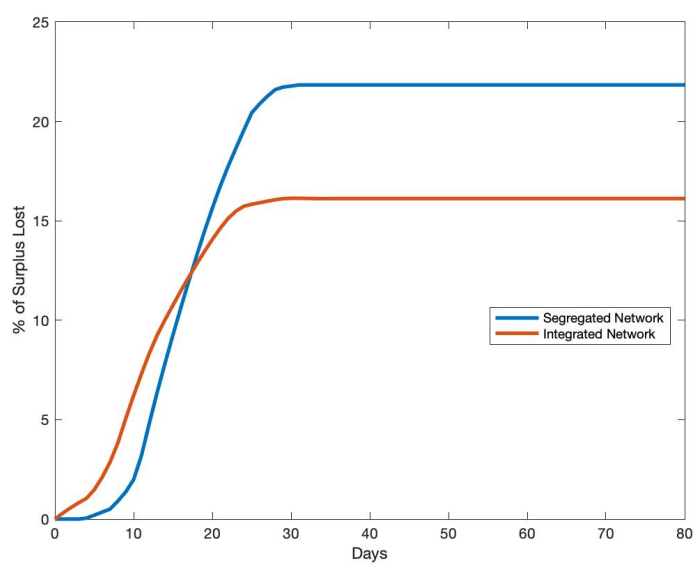

(c) Dynamic of Economic Cost

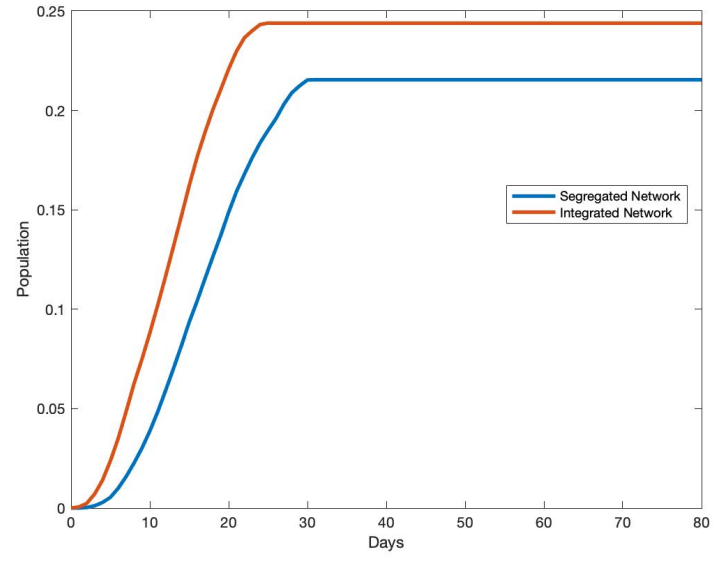

(b) Dynamic of Lockdown

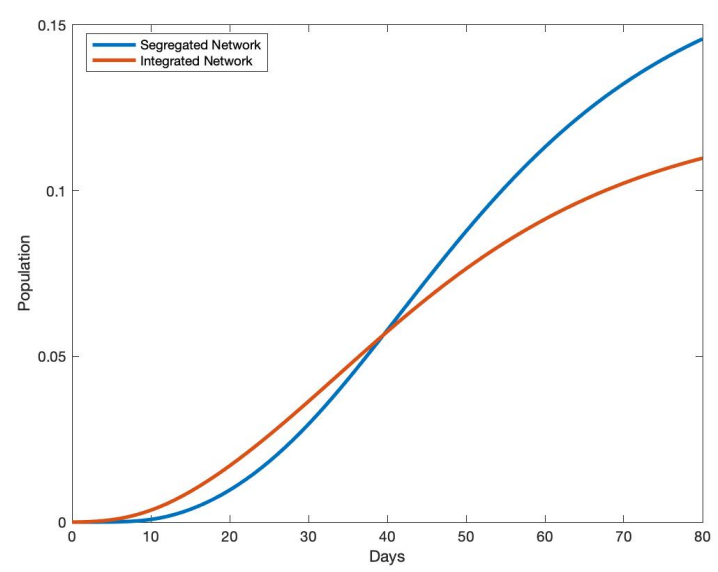

(d) Dynamic of Death 
Figure 36: Dynamics of Outcomes under the Optimal Lockdown Policy: Majority Group (6 to 15)

Patient Zero: Individual 7

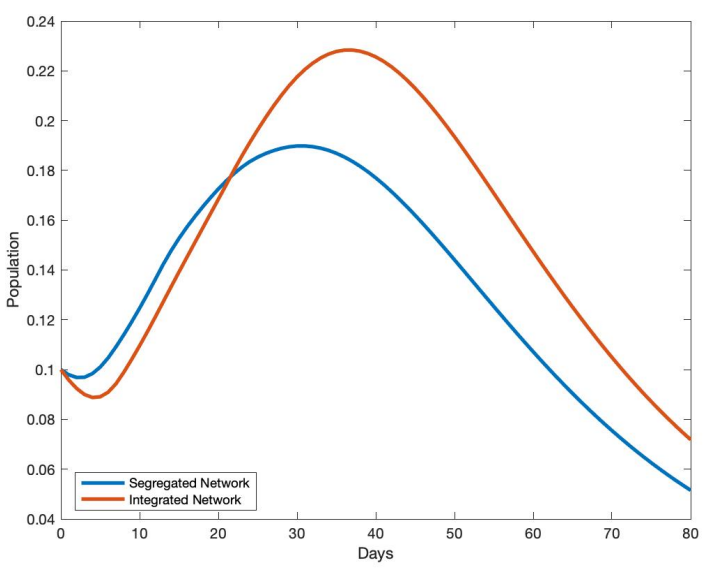

(a) Dynamic of Infection

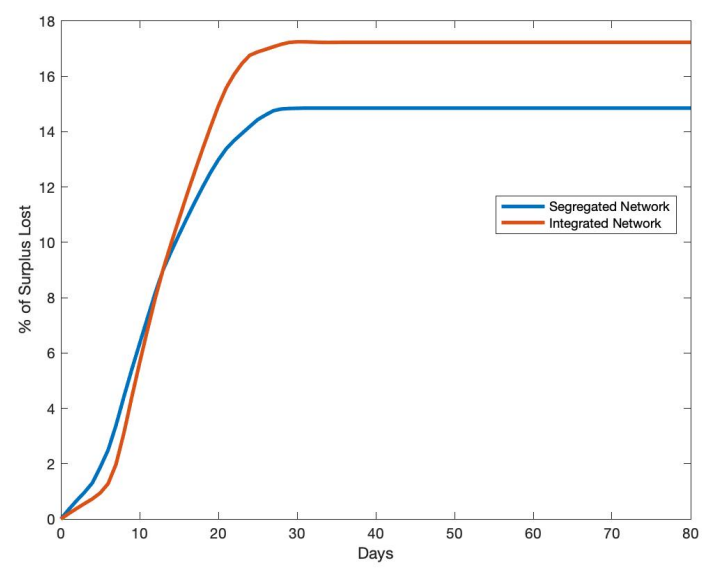

(c) Dynamic of Economic Cost

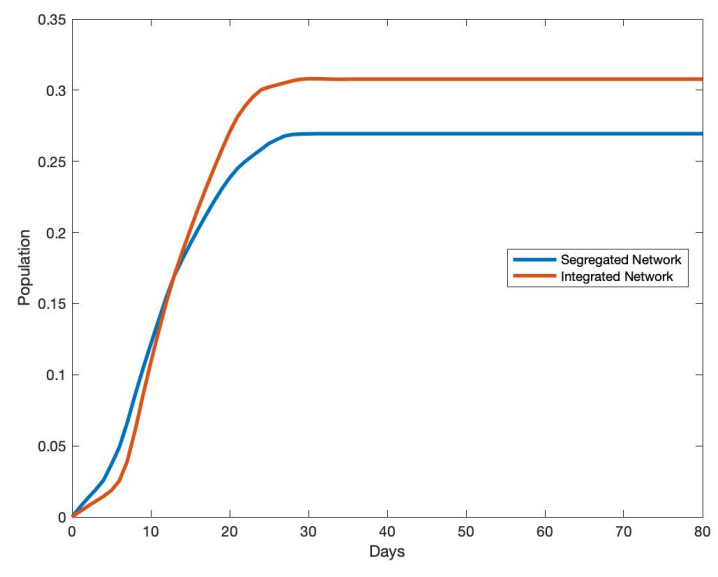

(b) Dynamic of Lockdown

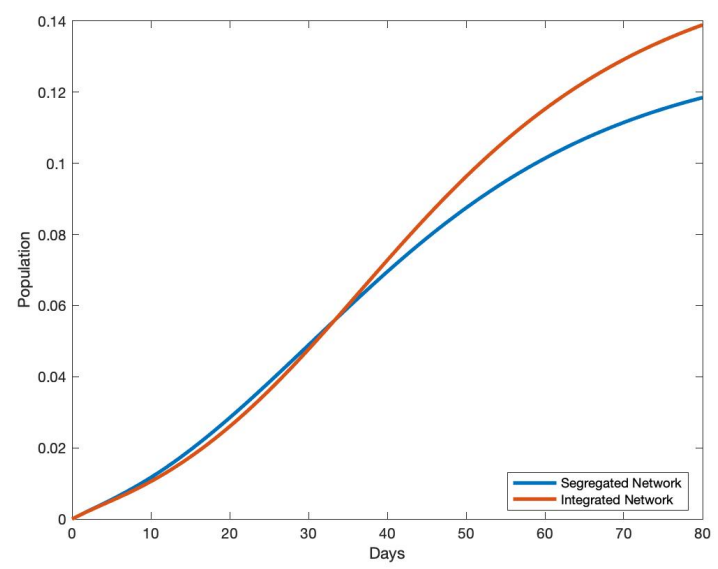

(d) Dynamic of Death 
Figure 37: Effect of Segregation on Minority-Majority Differences in Outcomes: Diff-in-Diffs Analysis

Patient Zero: Individual 7

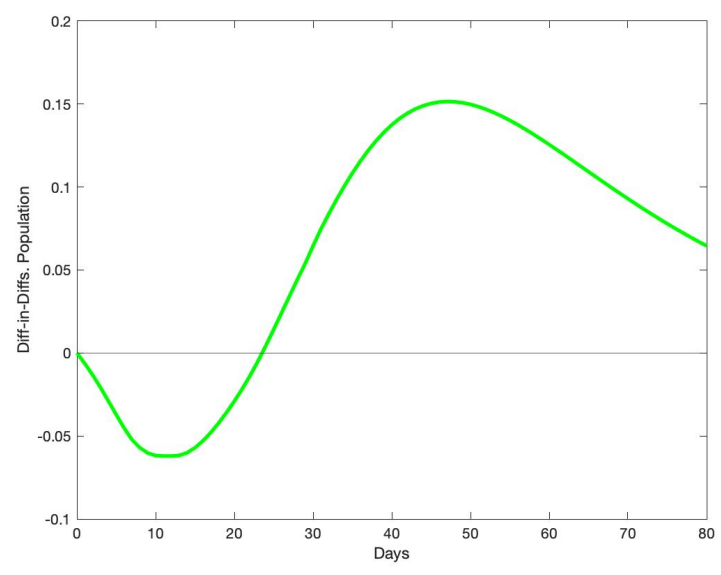

(a) Infection

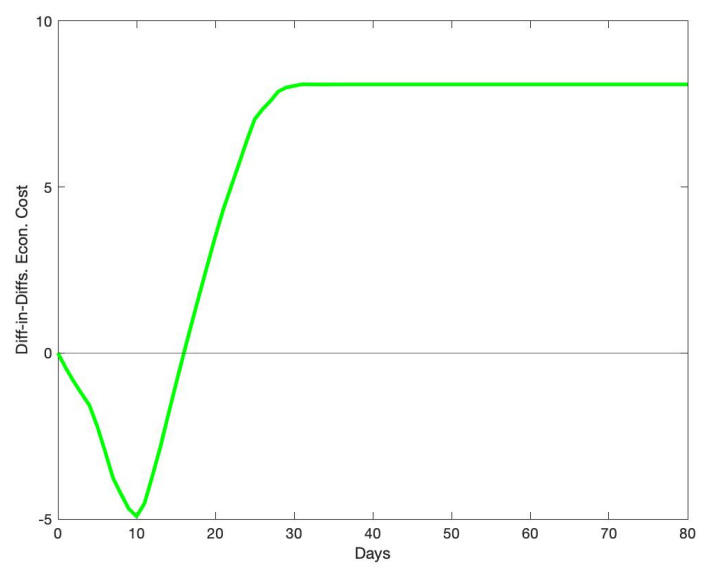

(c) Economic Cost

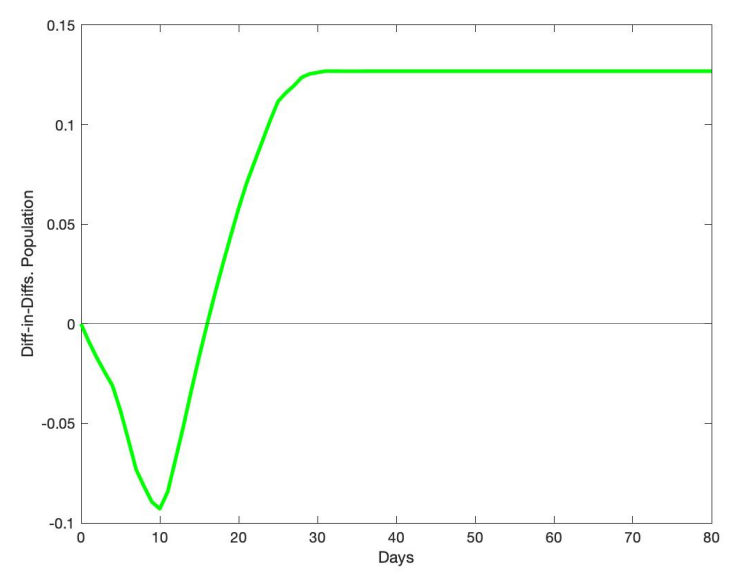

(b) Lockdown

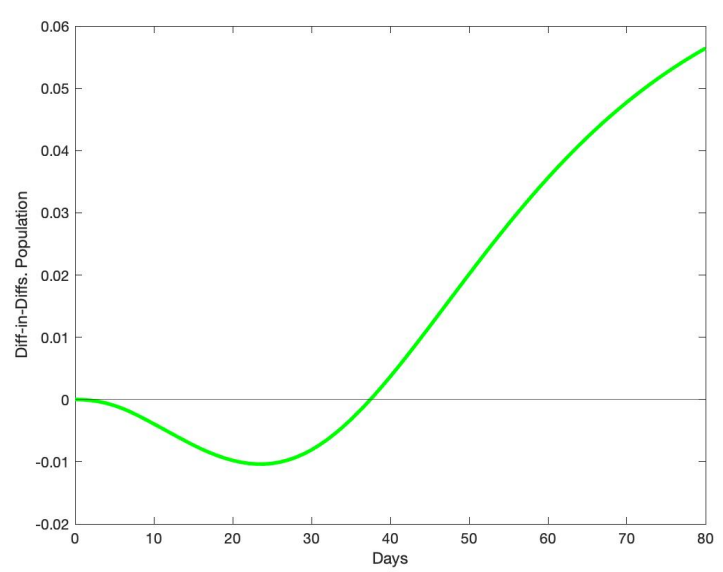

(d) Death 
Figure 38: Dynamics of Outcomes under the Optimal Lockdown Policy: Whole Population Patient Zero: Individual 8

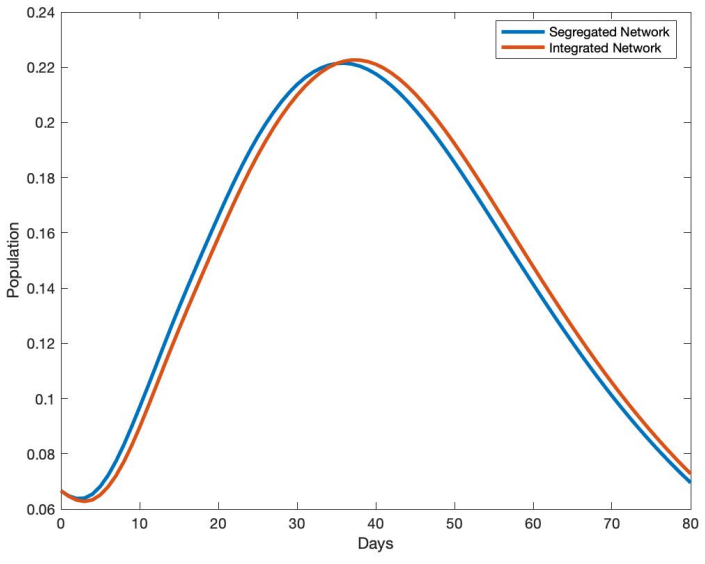

(a) Dynamic of Infection

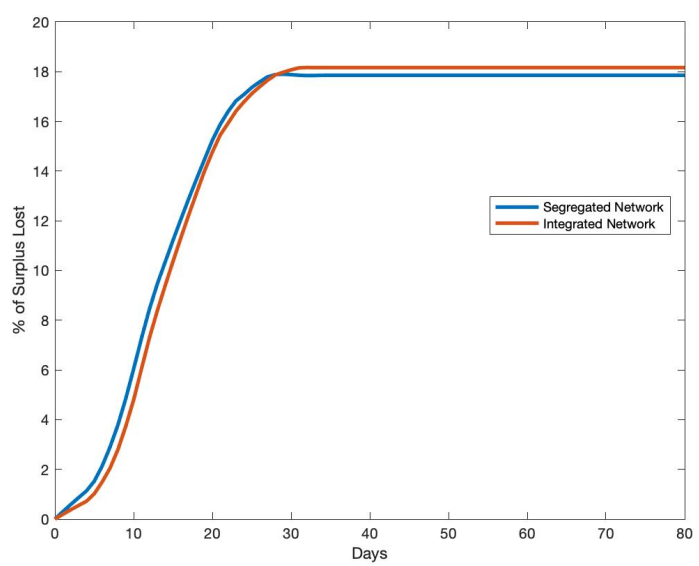

(c) Dynamic of Economic Cost

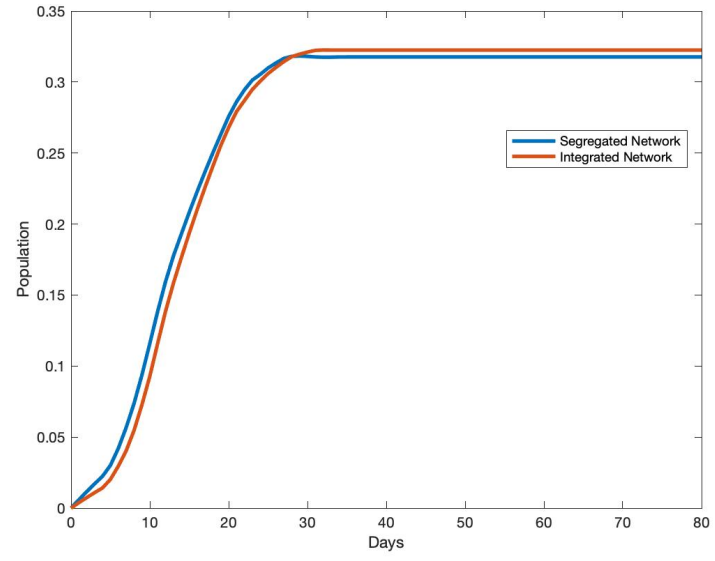

(b) Dynamic of Lockdown

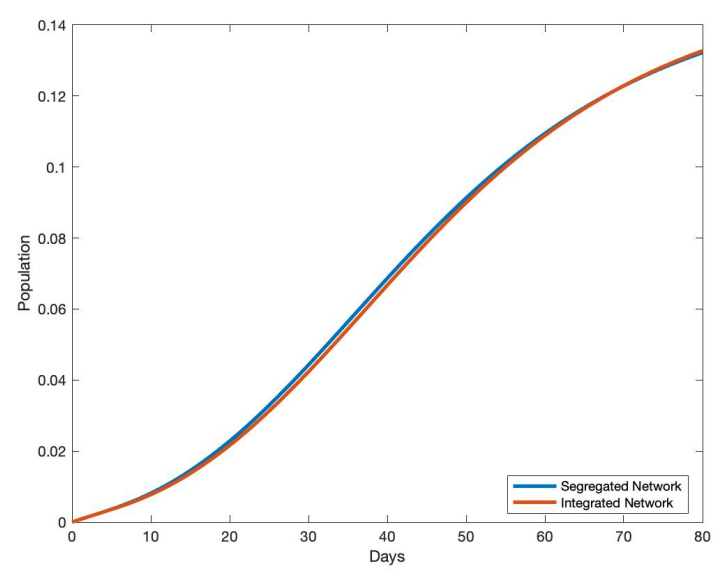

(d) Dynamic of Death 
Figure 39: Dynamics of Outcomes under the Optimal Lockdown Policy: Minority Group (1 to 5) Patient Zero: Individual 8

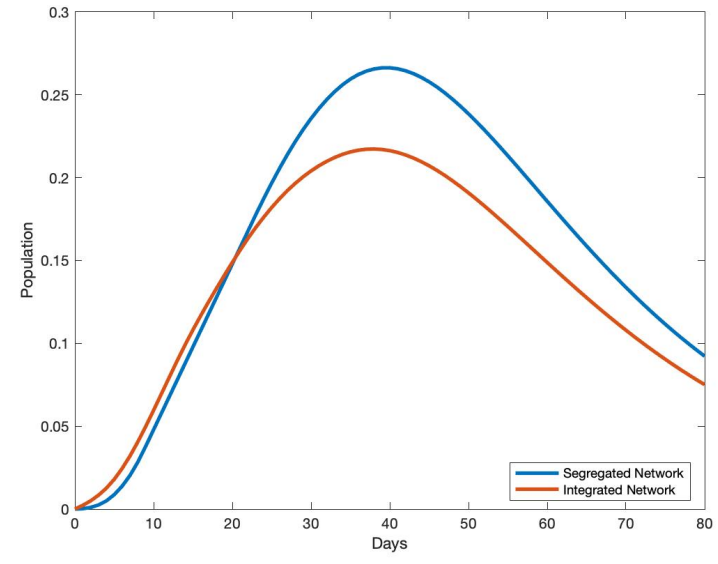

(a) Dynamic of Infection

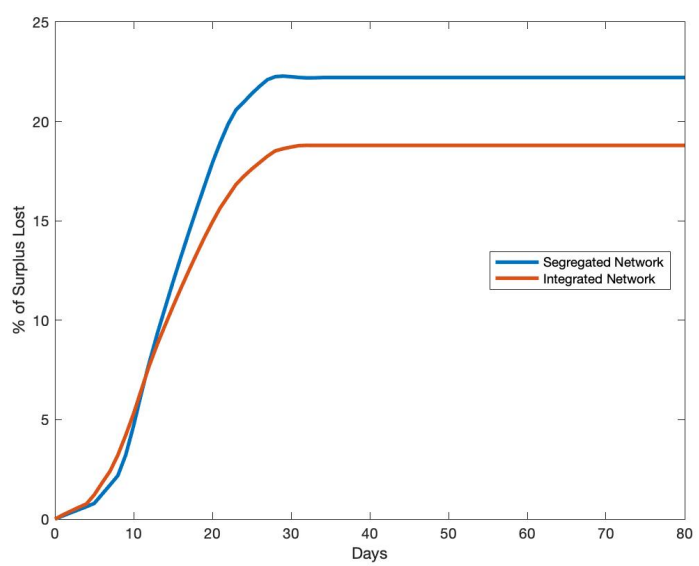

(c) Dynamic of Economic Cost

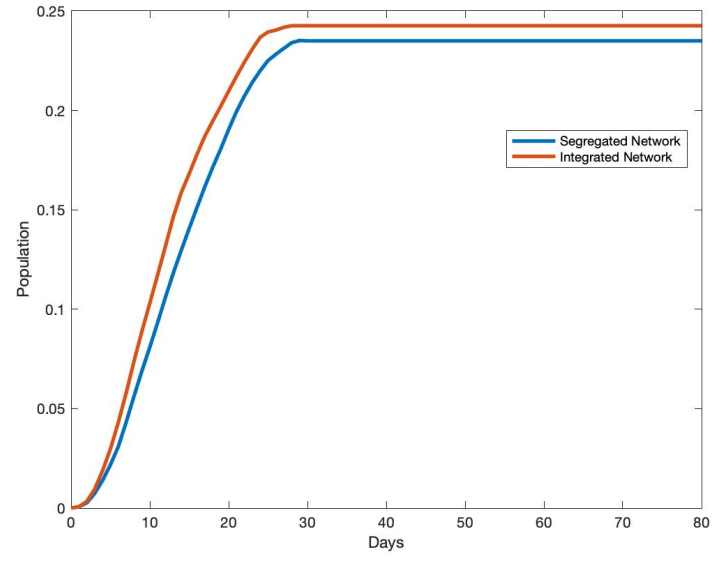

(b) Dynamic of Lockdown

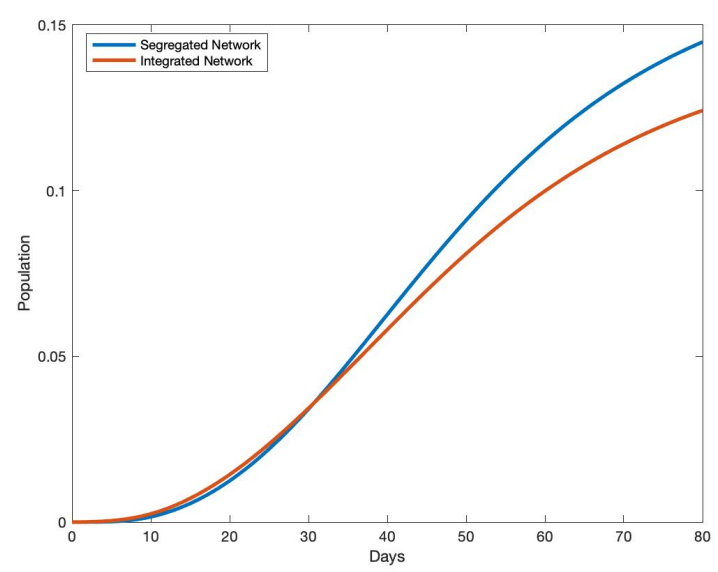

(d) Dynamic of Death 
Figure 40: Dynamics of Outcomes under the Optimal Lockdown Policy: Majority Group (6 to 15)

Patient Zero: Individual 8

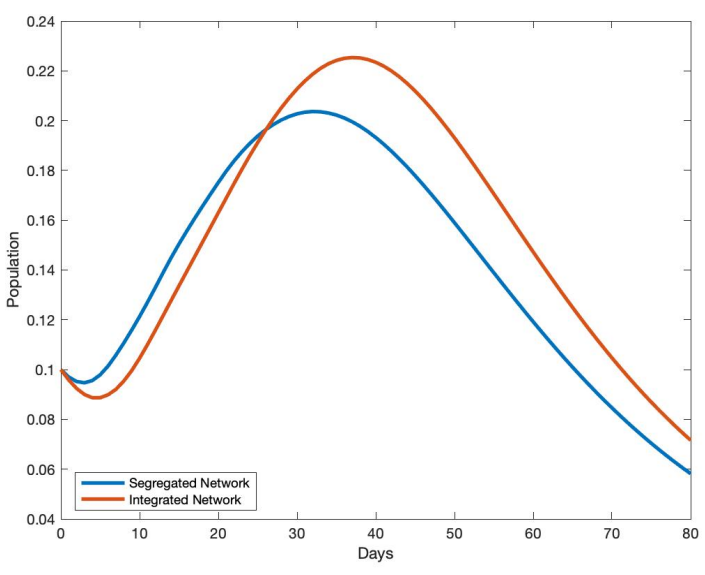

(a) Dynamic of Infection

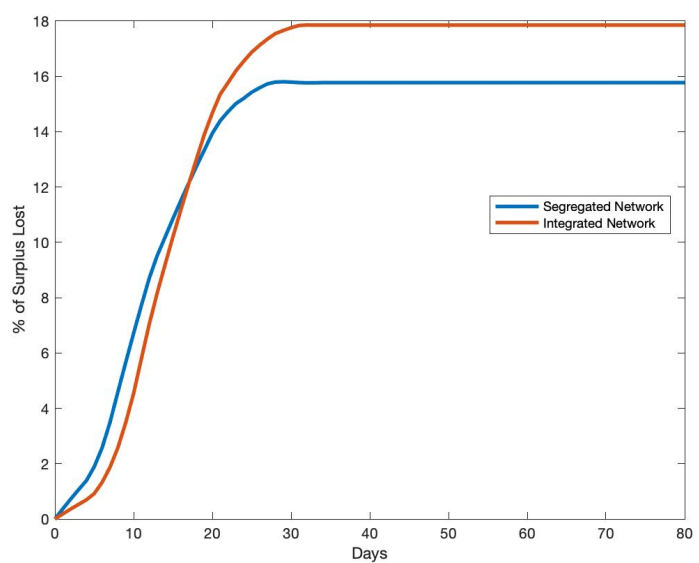

(c) Dynamic of Economic Cost

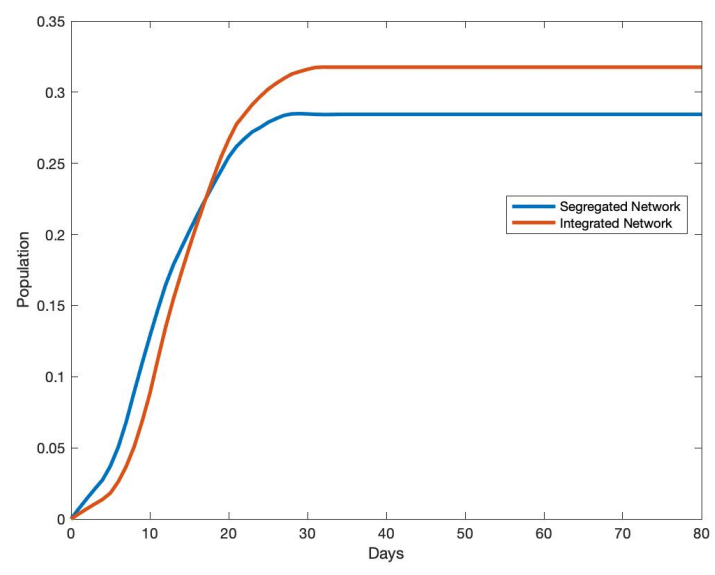

(b) Dynamic of Lockdown

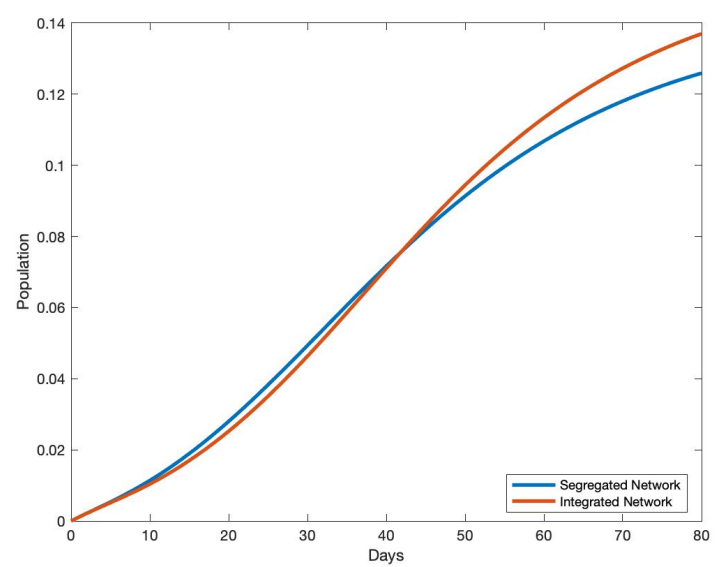

(d) Dynamic of Death 
Figure 41: Effect of Segregation on Minority-Majority Differences in Outcomes: Diff-in-Diffs Analysis

Patient Zero: Individual 8

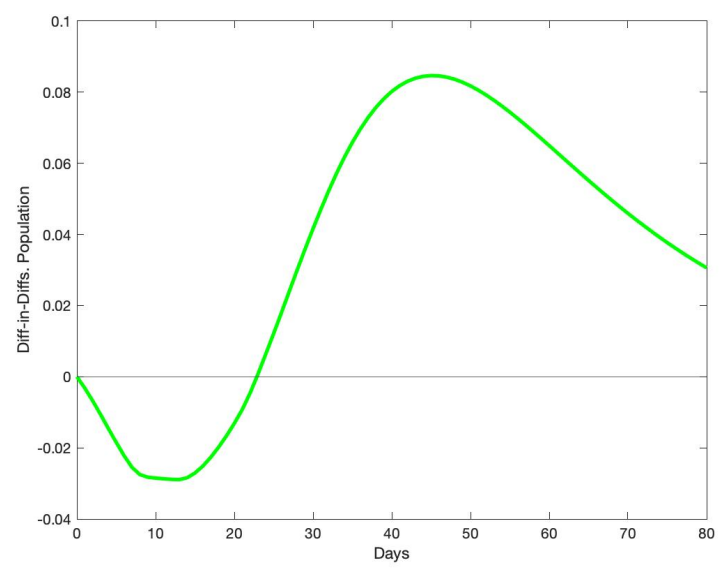

(a) Infection

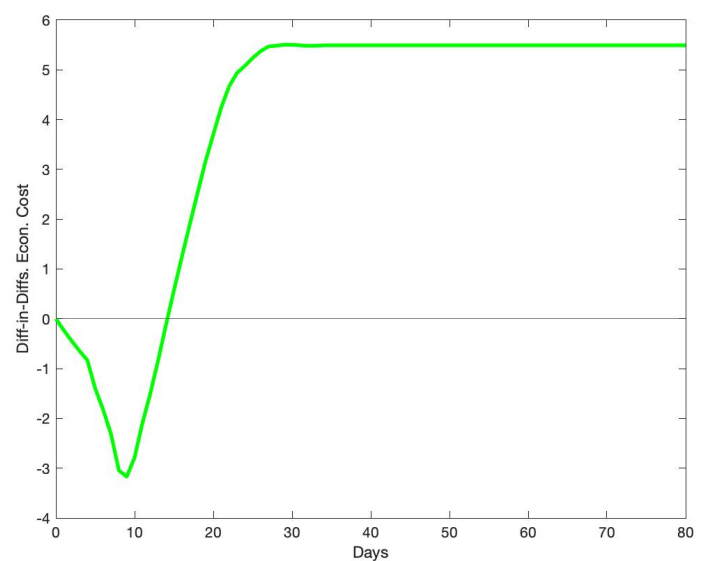

(c) Economic Cost

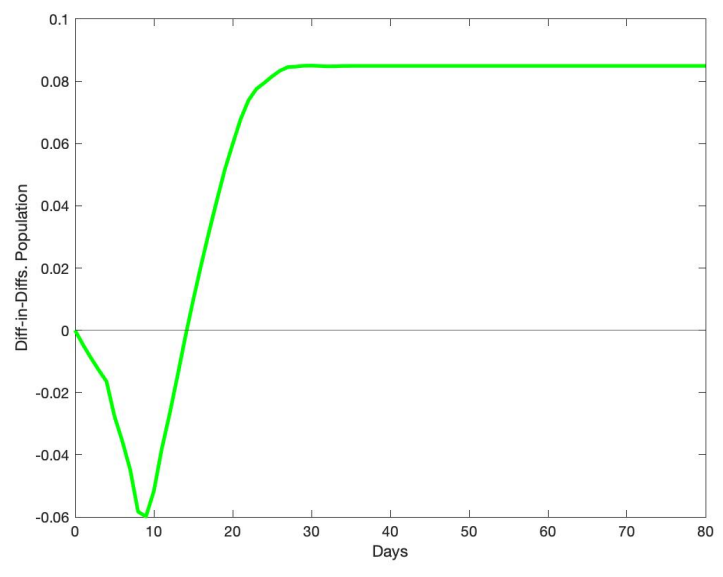

(b) Lockdown

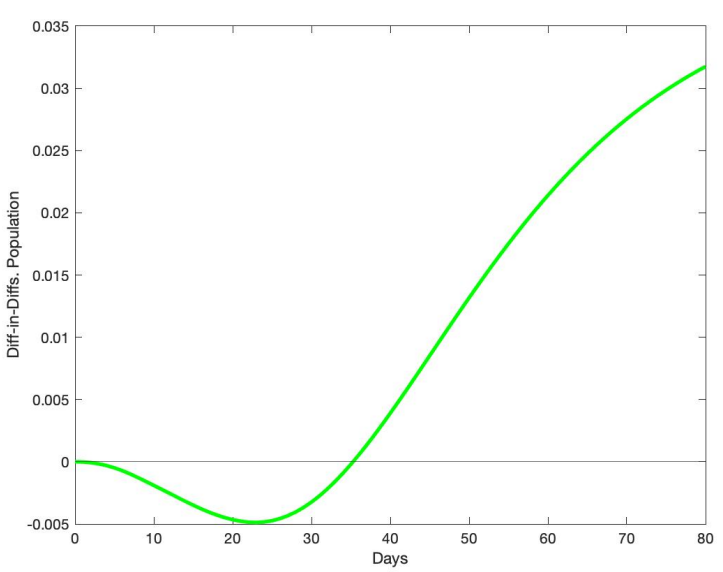

(d) Death 
Figure 42: Dynamics of Outcomes under the Optimal Lockdown Policy: Whole Population Patient Zero: Individual 9

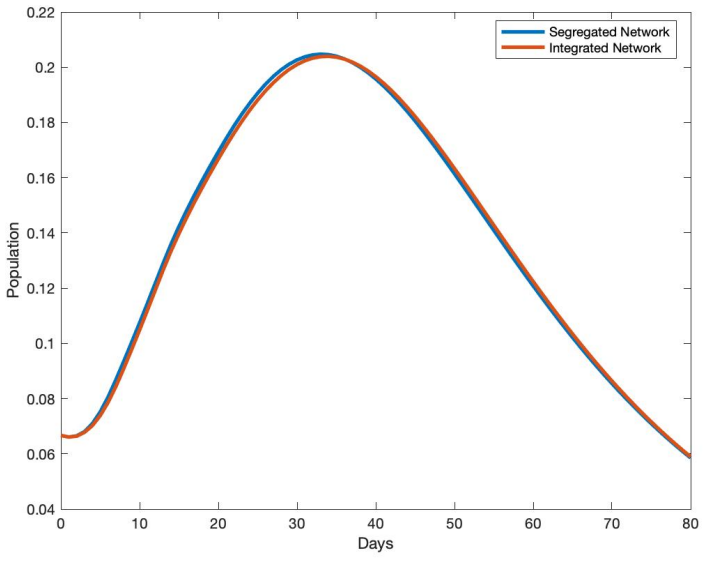

(a) Dynamic of Infection

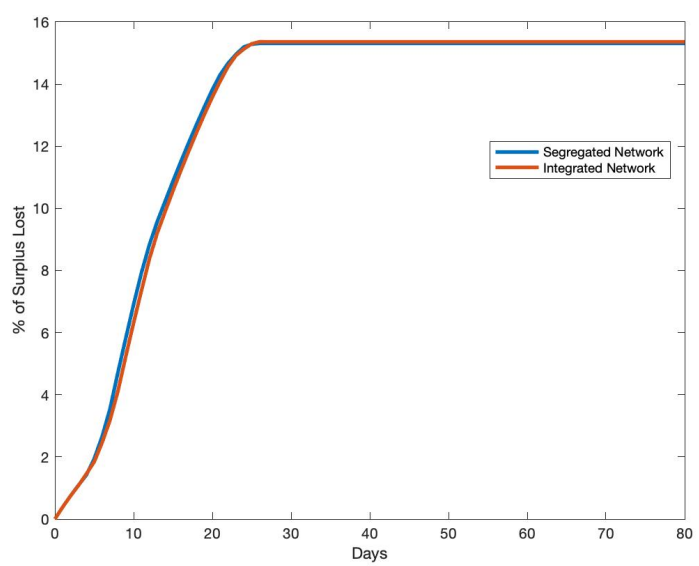

(c) Dynamic of Economic Cost

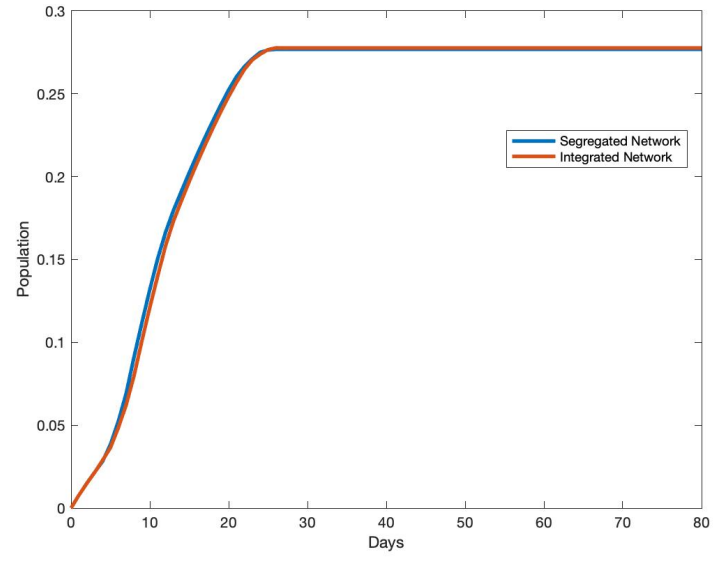

(b) Dynamic of Lockdown

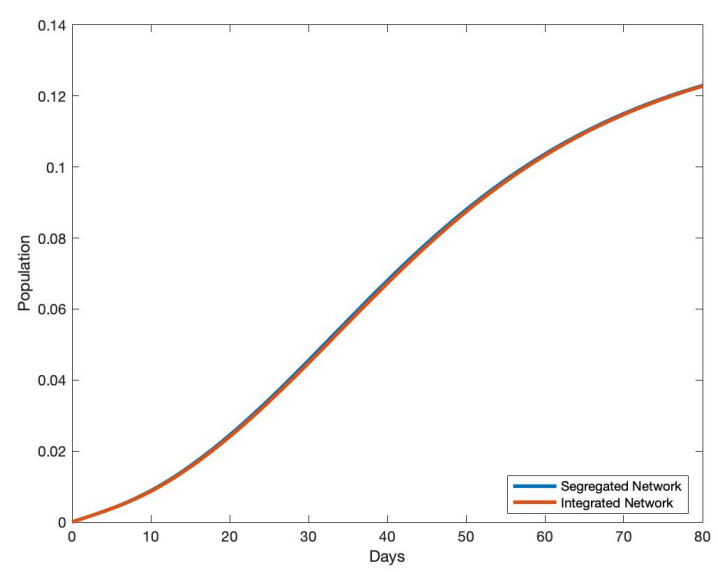

(d) Dynamic of Death 
Figure 43: Dynamics of Outcomes under the Optimal Lockdown Policy: Minority Group (1 to 5) Patient Zero: Individual 9

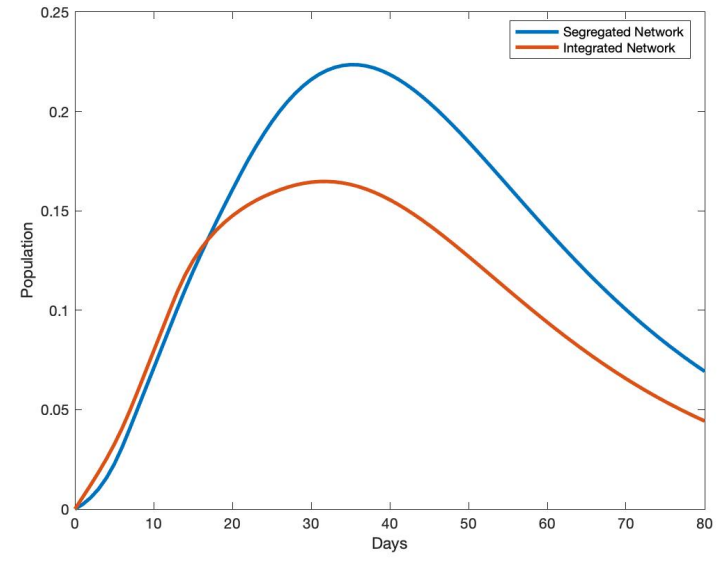

(a) Dynamic of Infection

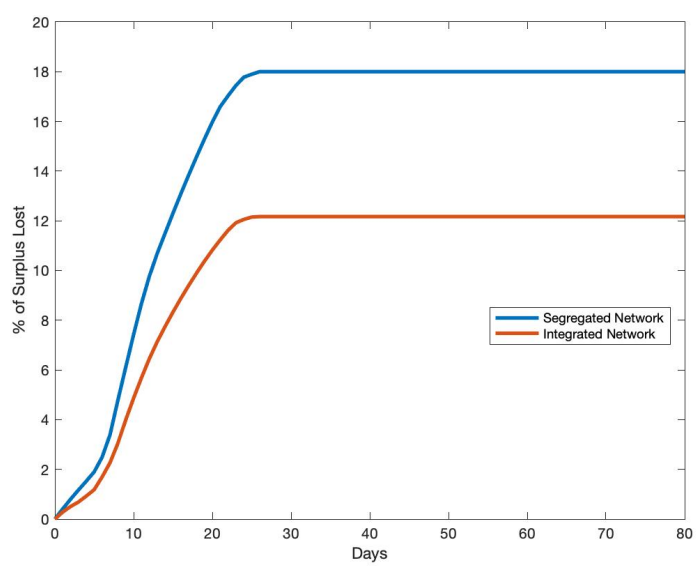

(c) Dynamic of Economic Cost

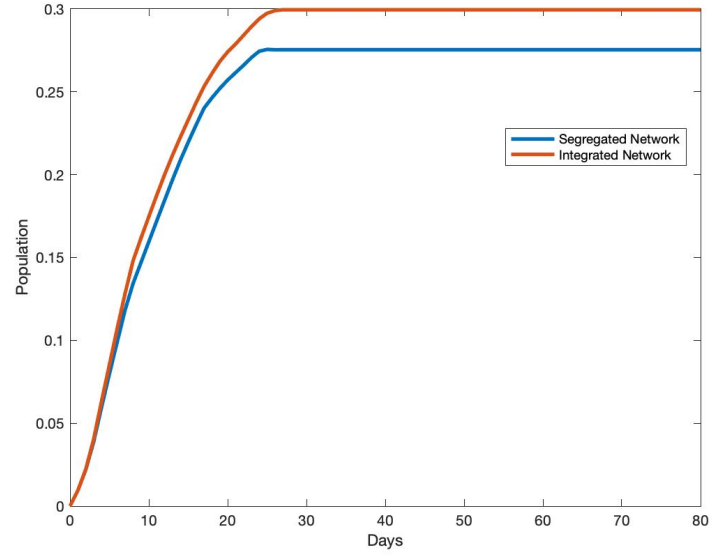

(b) Dynamic of Lockdown

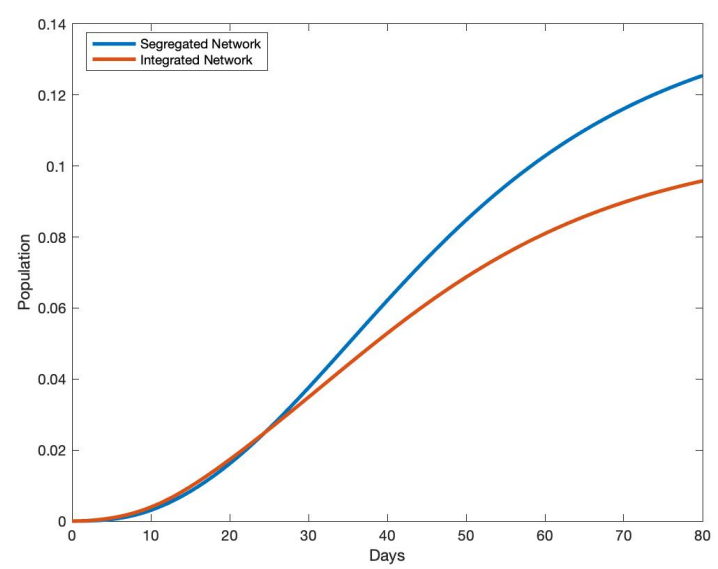

(d) Dynamic of Death 
Figure 44: Dynamics of Outcomes under the Optimal Lockdown Policy: Majority Group (6 to 15)

Patient Zero: Individual 9

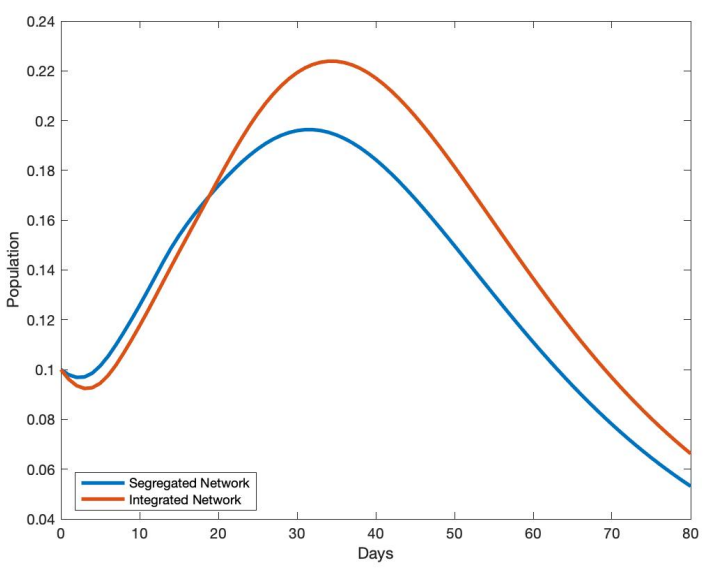

(a) Dynamic of Infection

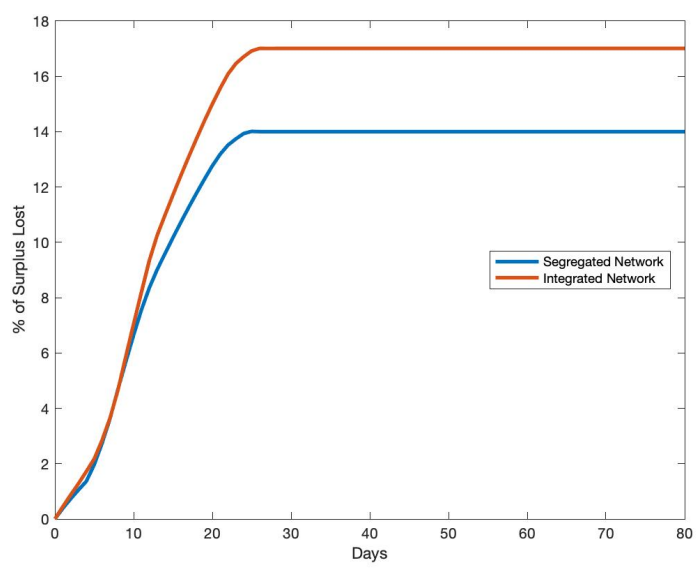

(c) Dynamic of Economic Cost

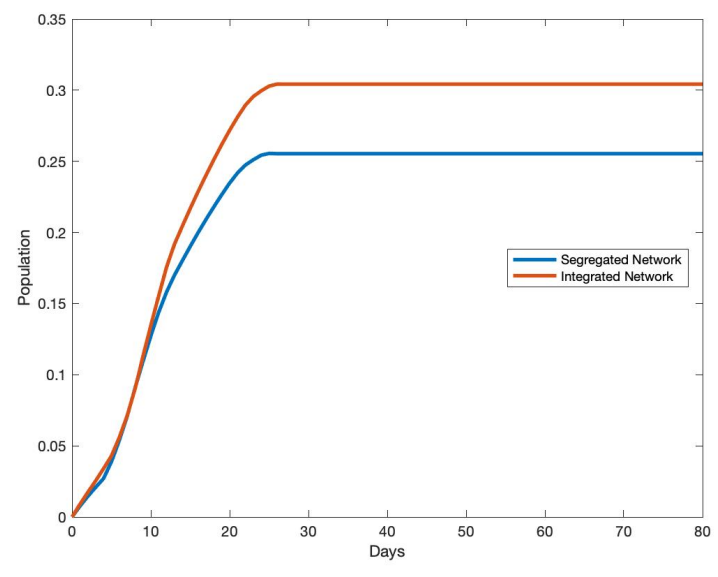

(b) Dynamic of Lockdown

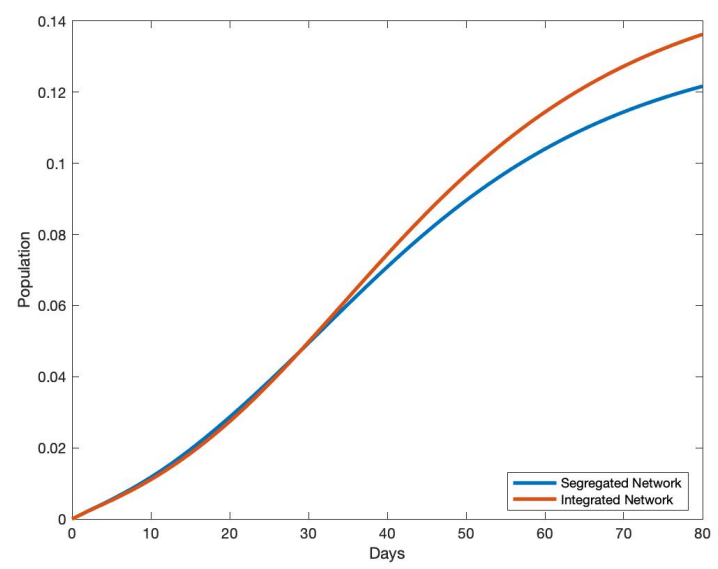

(d) Dynamic of Death 
Figure 45: Effect of Segregation on Minority-Majority Differences in Outcomes: Diff-in-Diffs Analysis

Patient Zero: Individual 9

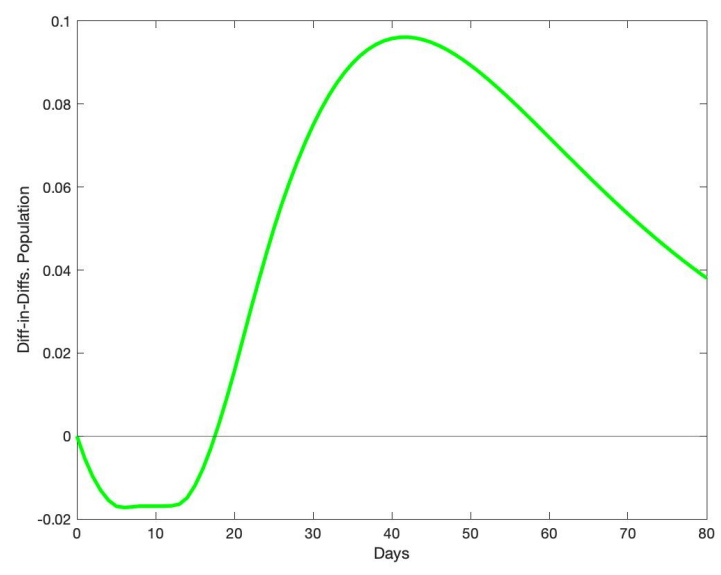

(a) Infection

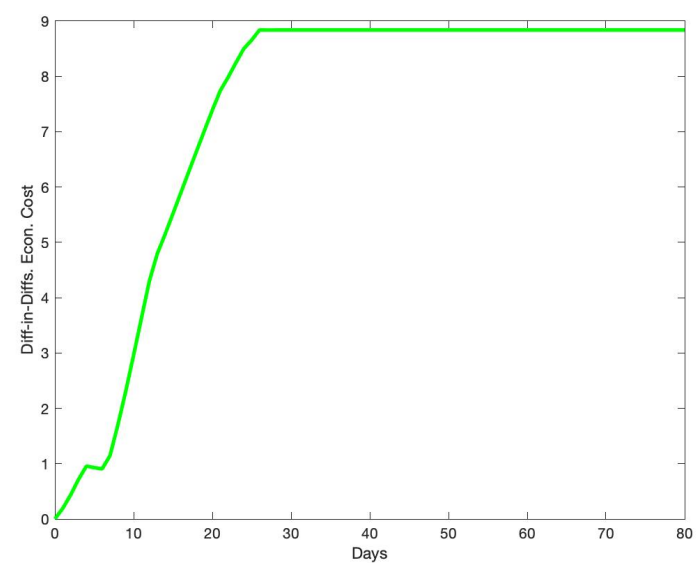

(c) Economic Cost

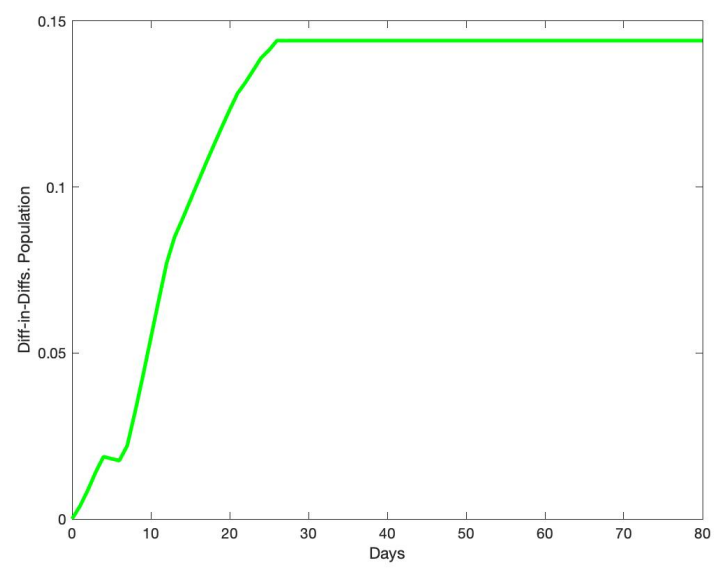

(b) Lockdown

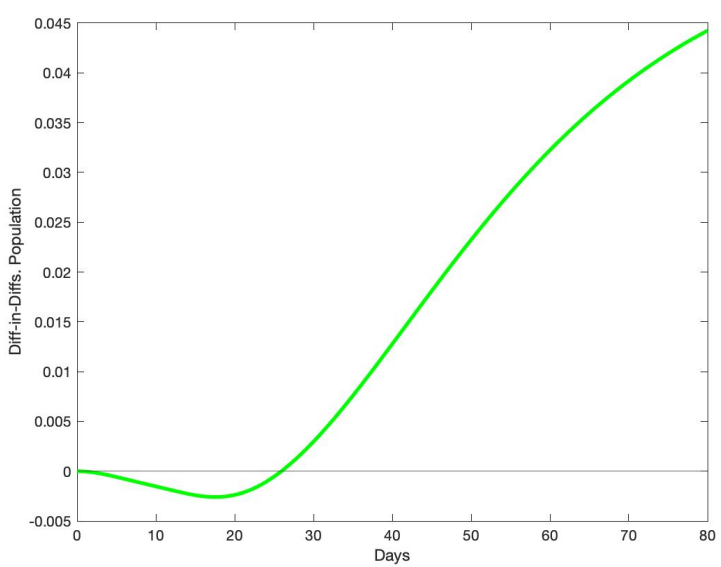

(d) Death 
Figure 46: Dynamics of Outcomes under the Optimal Lockdown Policy: Whole Population Patient Zero: Individual 10

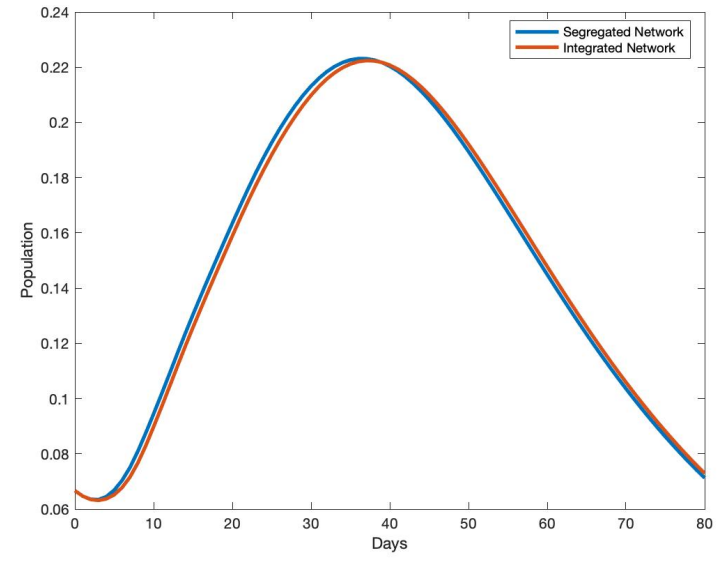

(a) Dynamic of Infection

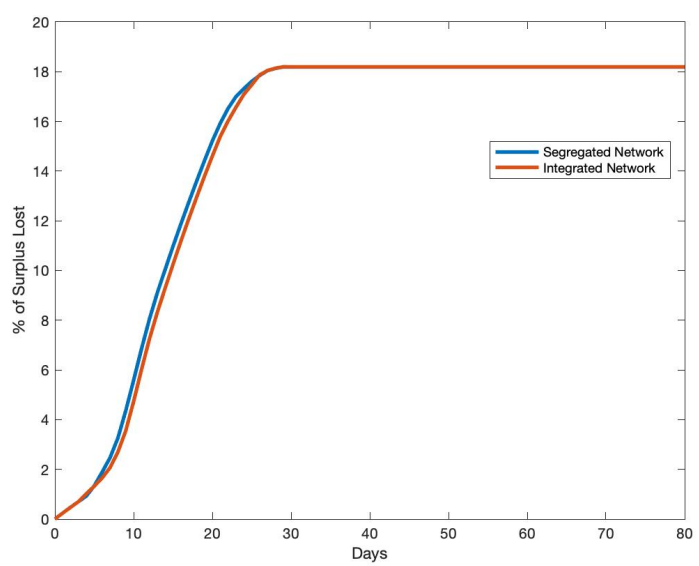

(c) Dynamic of Economic Cost

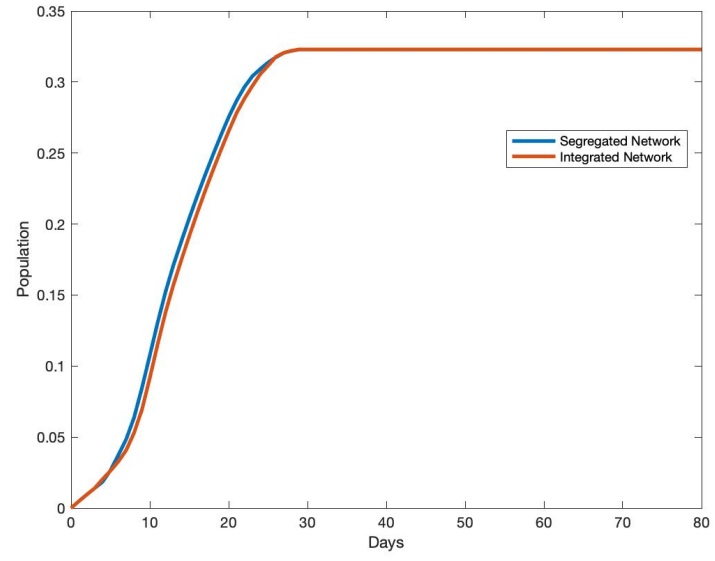

(b) Dynamic of Lockdown

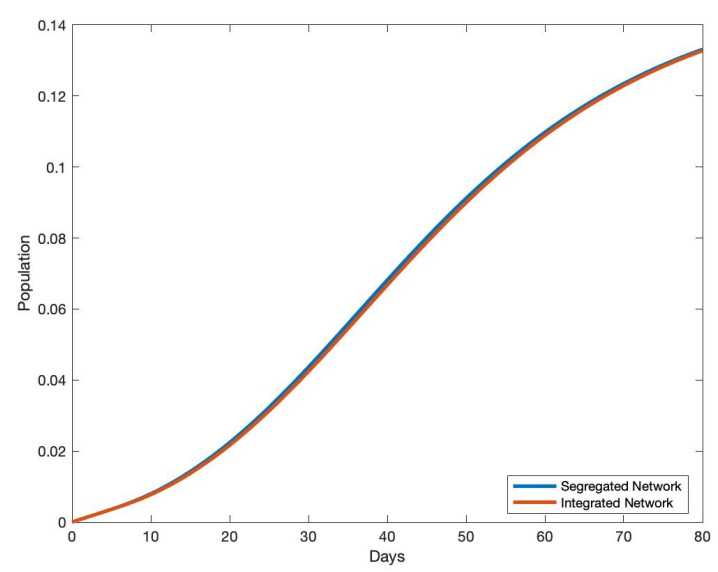

(d) Dynamic of Death 
Figure 47: Dynamics of Outcomes under the Optimal Lockdown Policy: Minority Group (1 to 5) Patient Zero: Individual 10

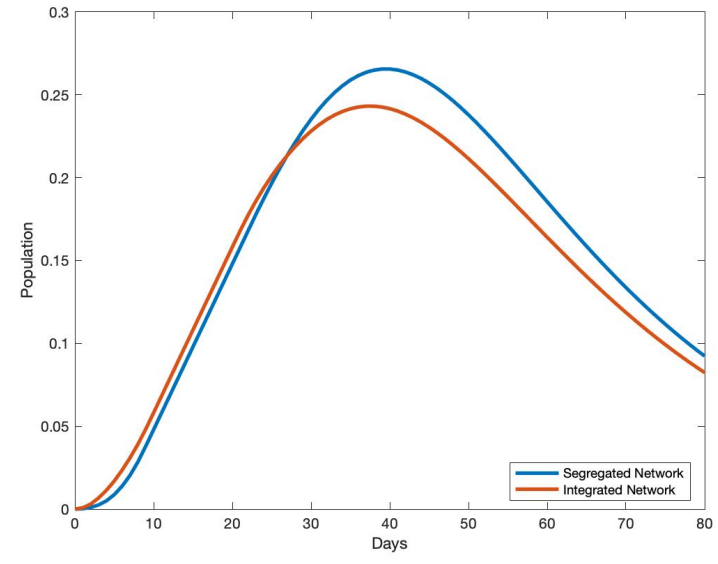

(a) Dynamic of Infection

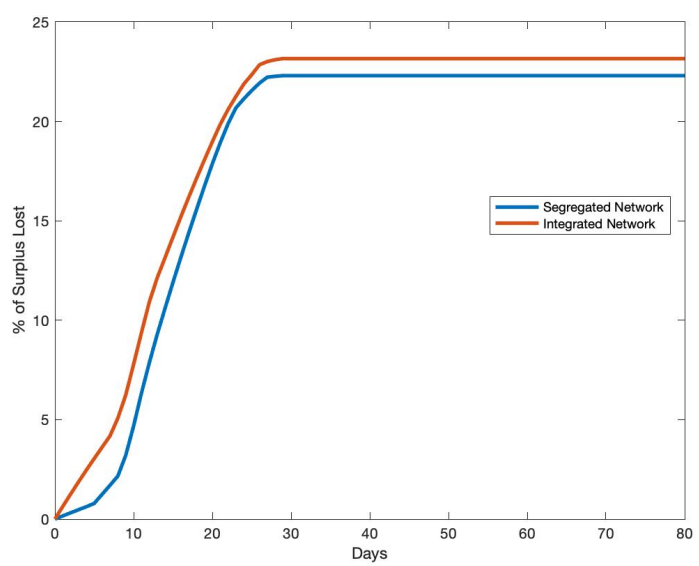

(c) Dynamic of Economic Cost

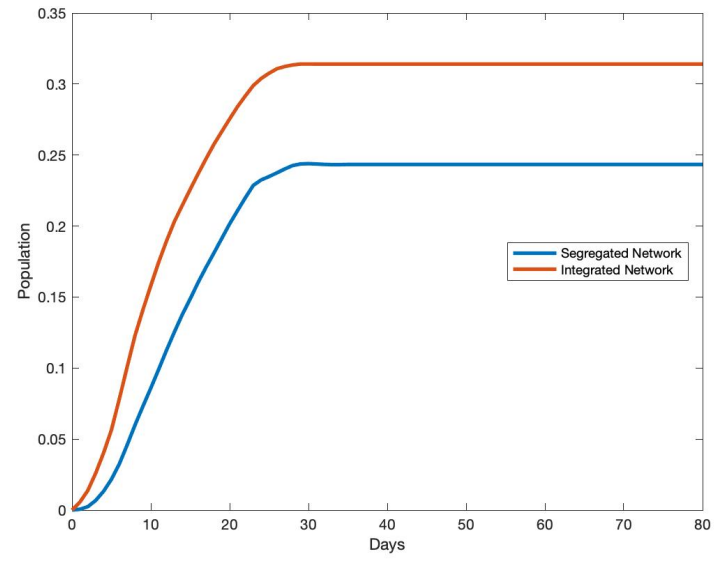

(b) Dynamic of Lockdown

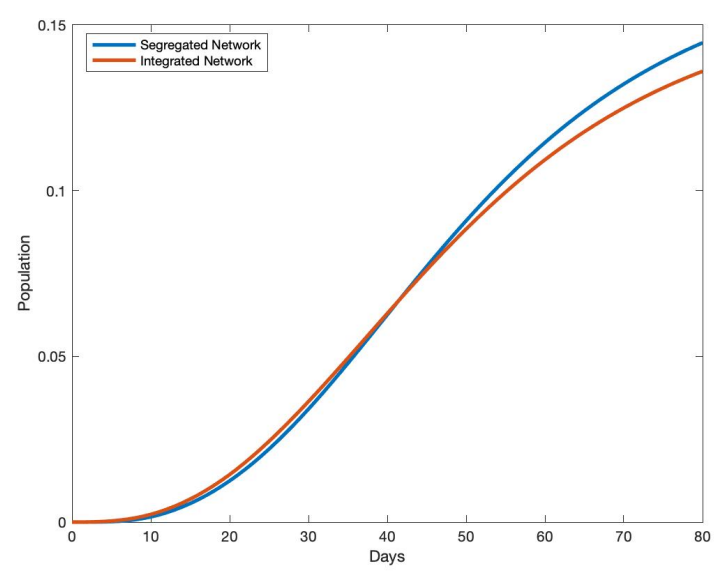

(d) Dynamic of Death 
Figure 48: Dynamics of Outcomes under the Optimal Lockdown Policy: Majority Group (6 to 15) Patient Zero: Individual 10

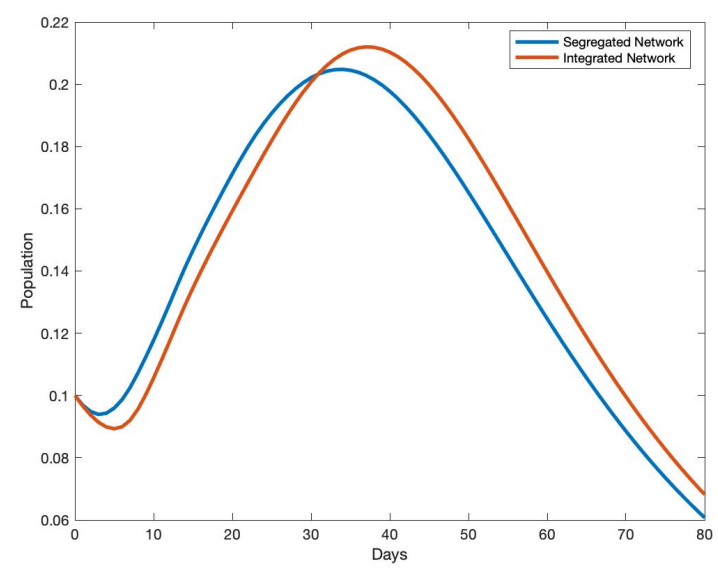

(a) Dynamic of Infection

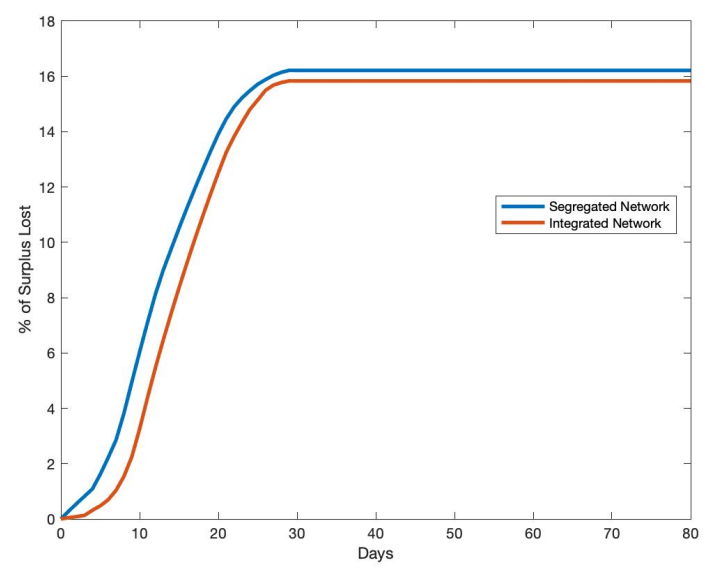

(c) Dynamic of Economic Cost

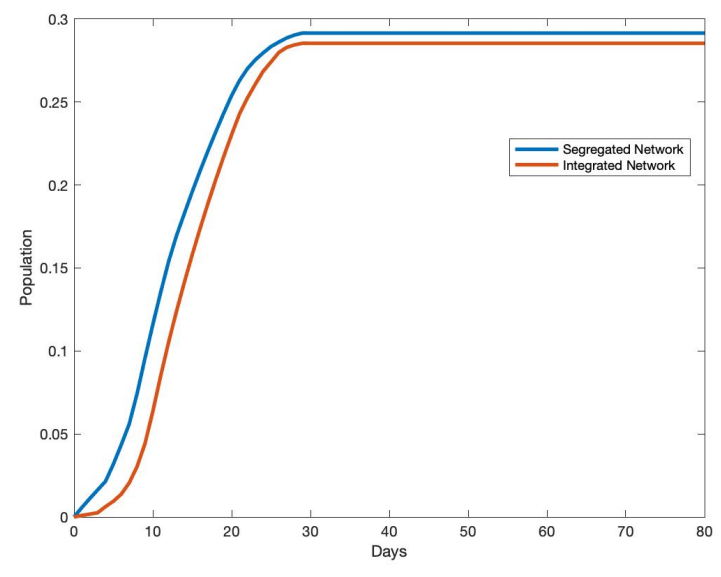

(b) Dynamic of Lockdown

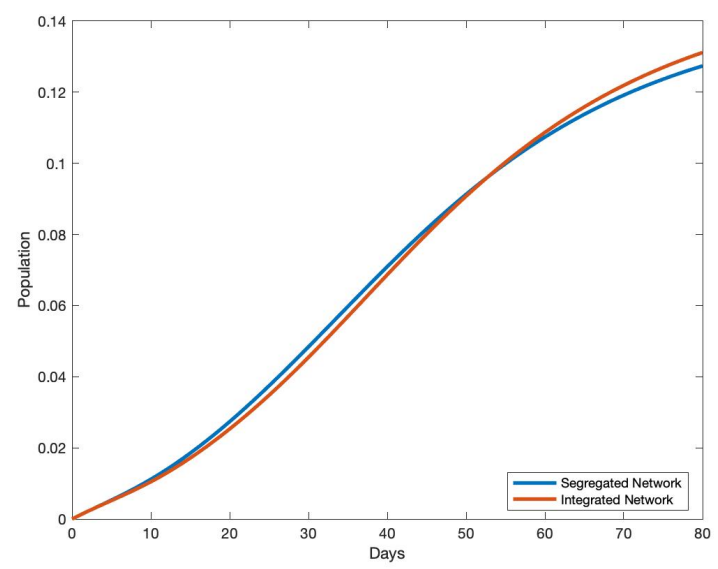

(d) Dynamic of Death 
Figure 49: Effect of Segregation on Minority-Majority Differences in Outcomes: Diff-in-Diffs Analysis

Patient Zero: Individual 10

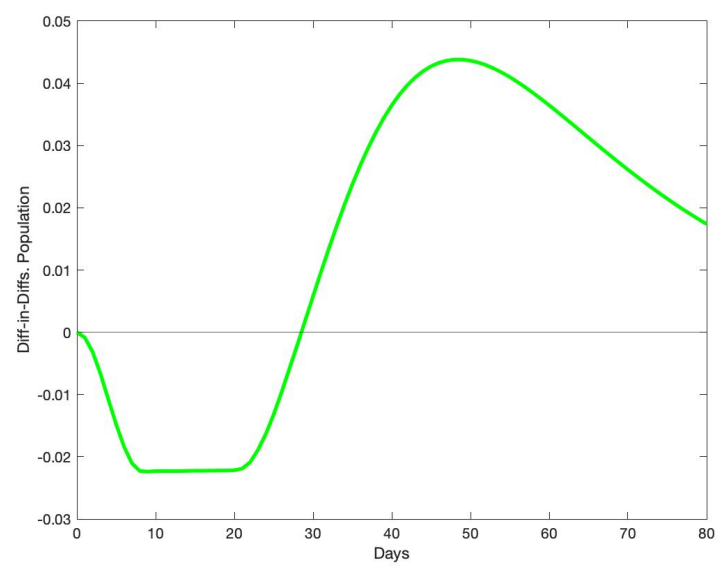

(a) Infection

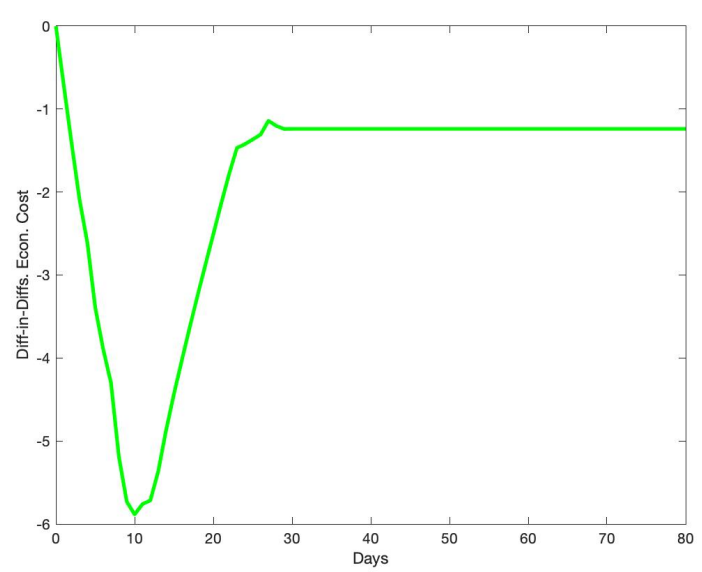

(c) Economic Cost

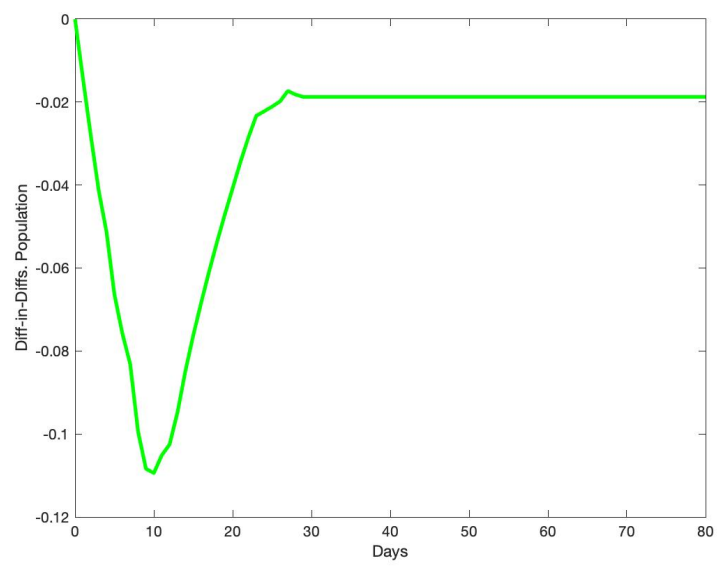

(b) Lockdown

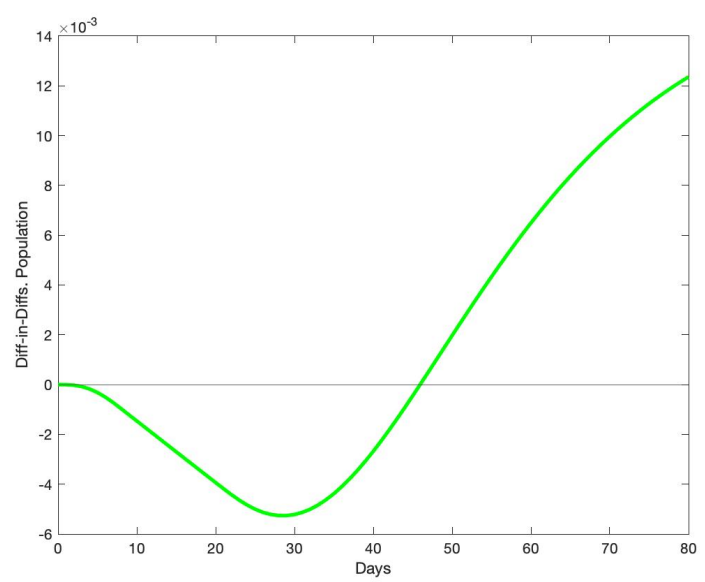

(d) Death 
Figure 50: Dynamics of Outcomes under the Optimal Lockdown Policy: Whole Population Patient Zero: Individual 11

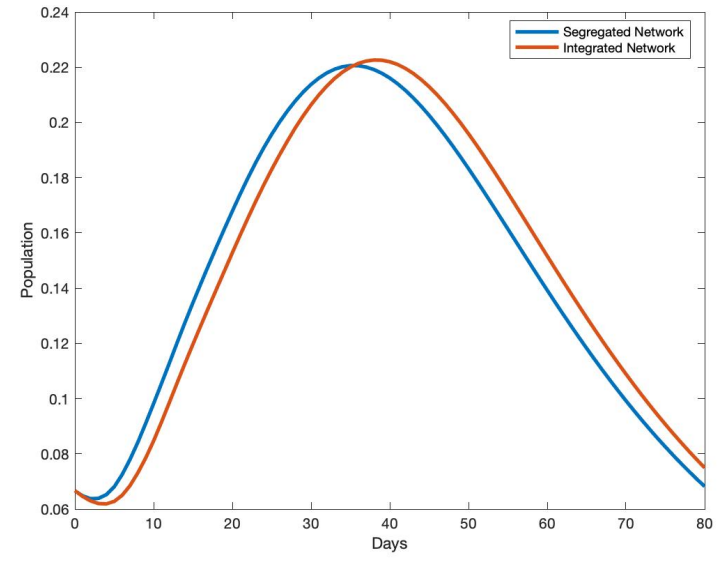

(a) Dynamic of Infection

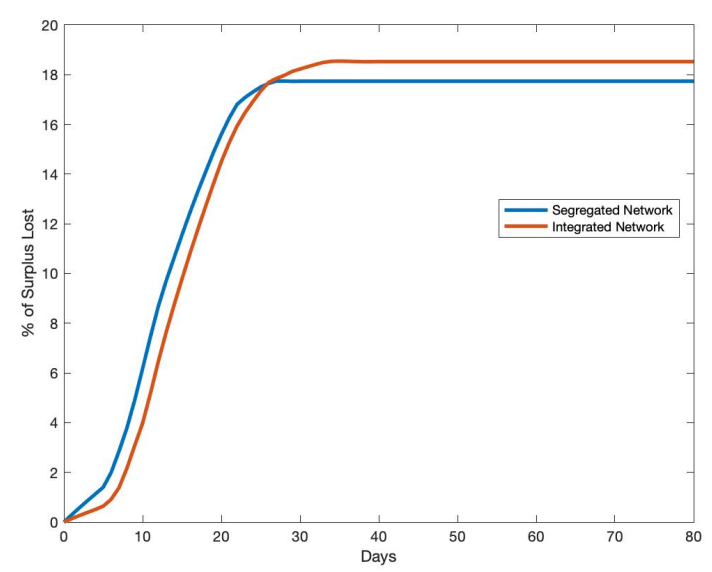

(c) Dynamic of Economic Cost

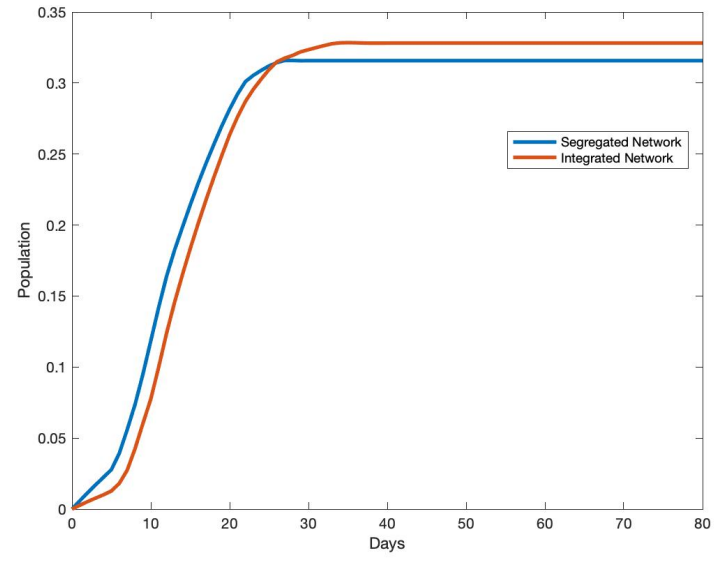

(b) Dynamic of Lockdown

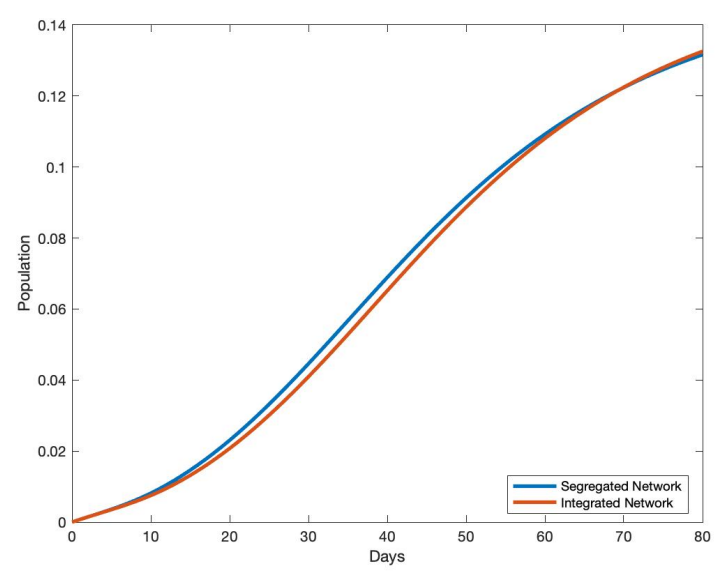

(d) Dynamic of Death 
Figure 51: Dynamics of Outcomes under the Optimal Lockdown Policy: Minority Group (1 to 5) Patient Zero: Individual 11

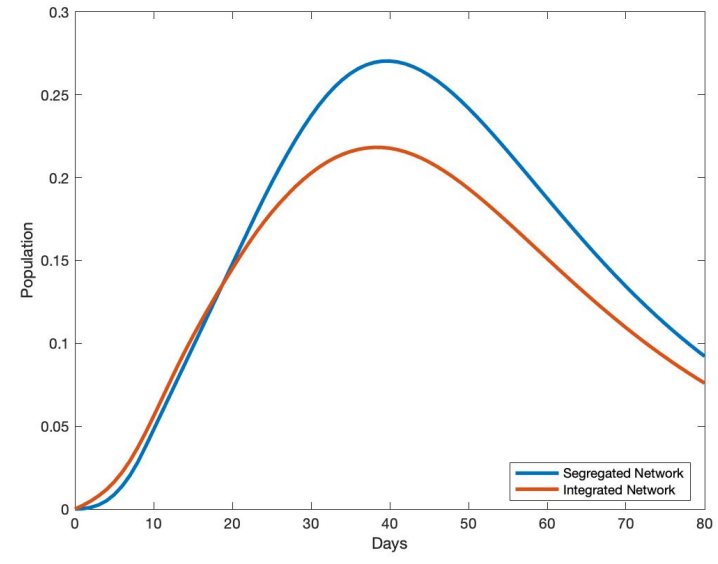

(a) Dynamic of Infection

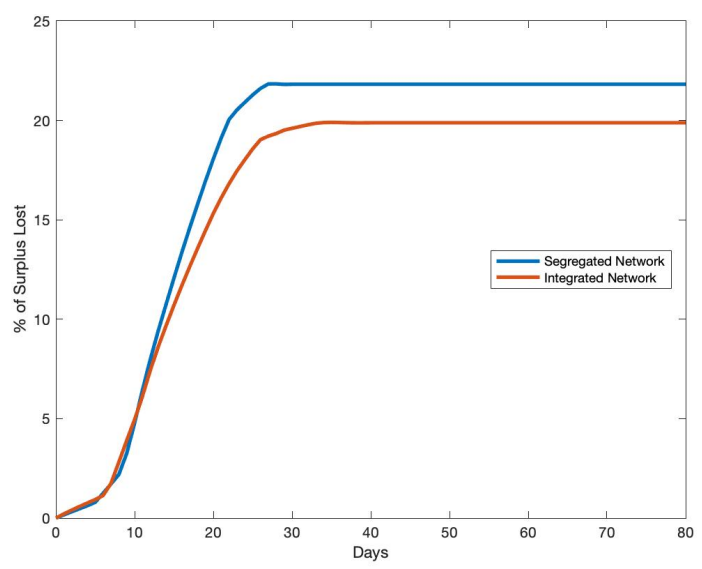

(c) Dynamic of Economic Cost

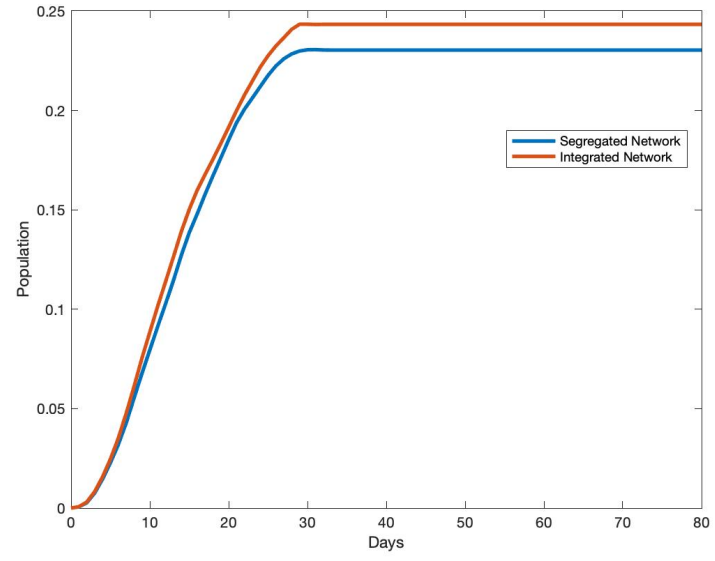

(b) Dynamic of Lockdown

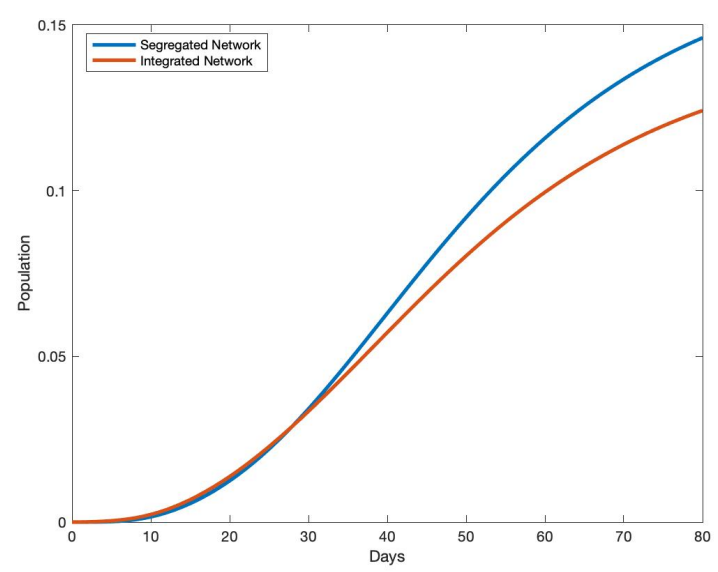

(d) Dynamic of Death 
Figure 52: Dynamics of Outcomes under the Optimal Lockdown Policy: Majority Group (6 to 15)

Patient Zero: Individual 11

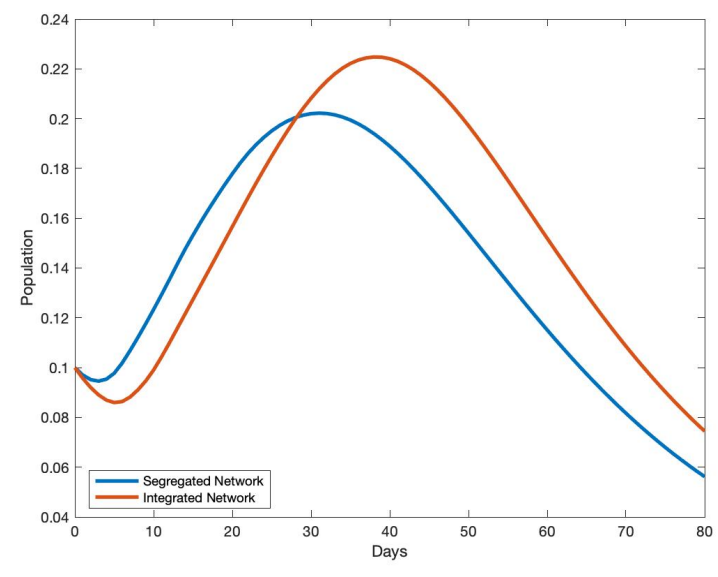

(a) Dynamic of Infection

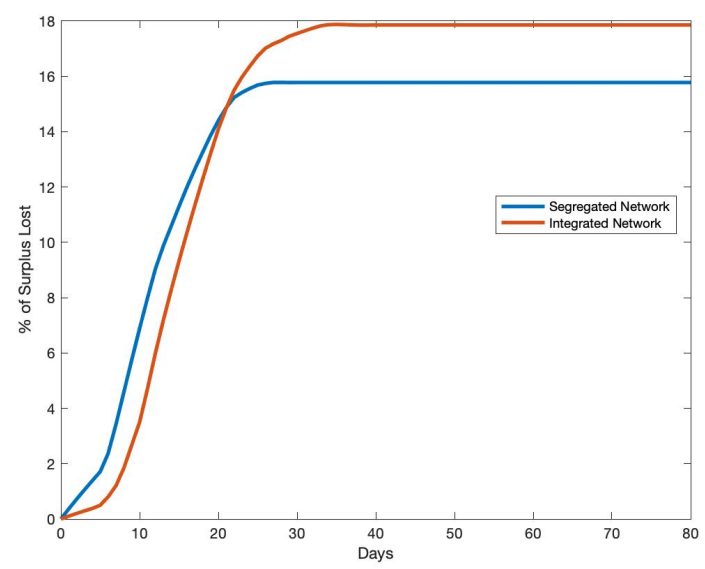

(c) Dynamic of Economic Cost

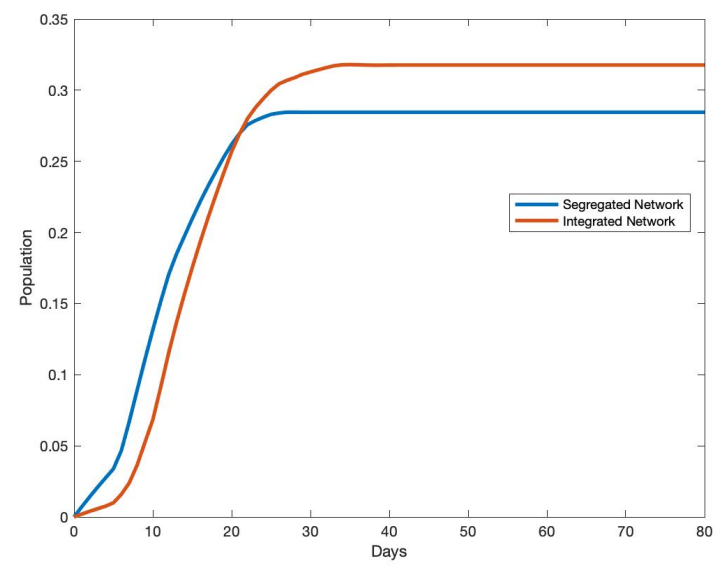

(b) Dynamic of Lockdown

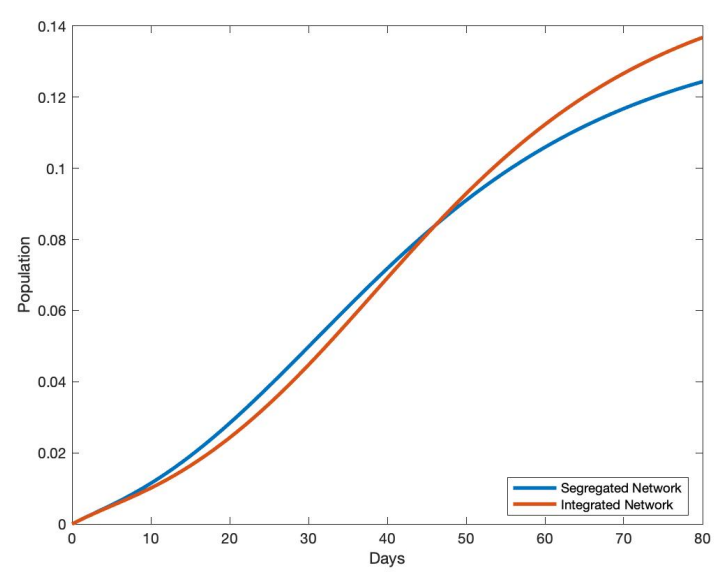

(d) Dynamic of Death 
Figure 53: Effect of Segregation on Minority-Majority Differences in Outcomes: Diff-in-Diffs Analysis

Patient Zero: Individual 11

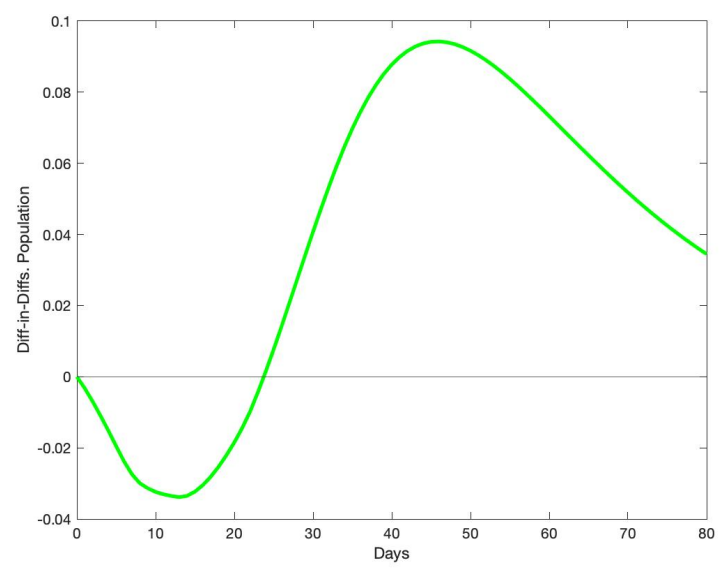

(a) Infection

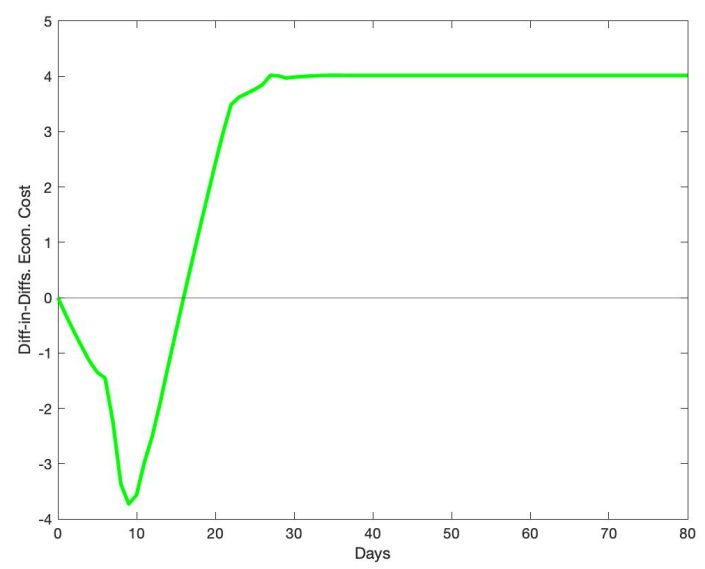

(c) Economic Cost

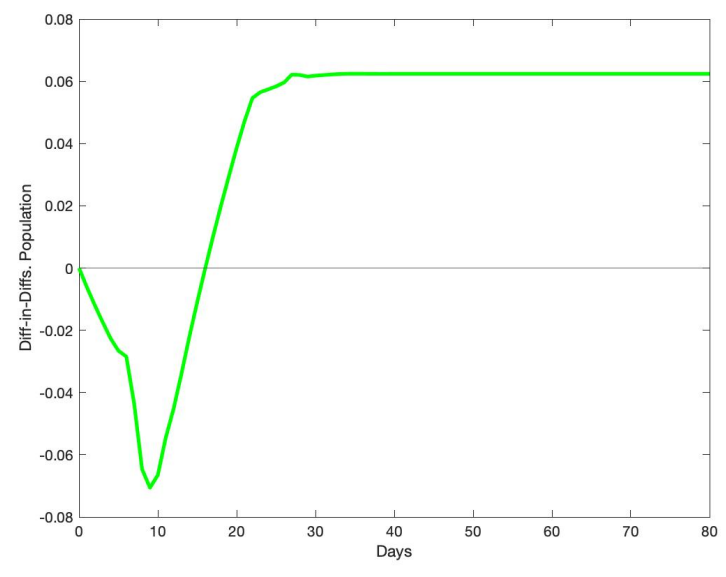

(b) Lockdown

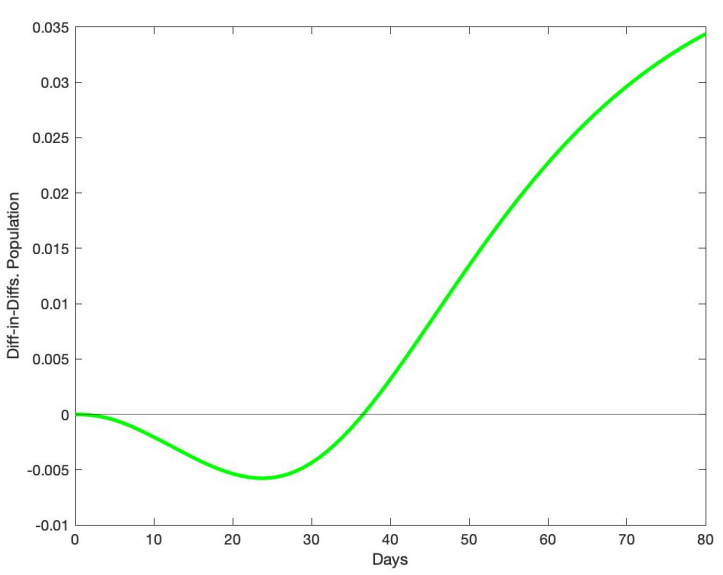

(d) Death 
Figure 54: Dynamics of Outcomes under the Optimal Lockdown Policy: Whole Population Patient Zero: Individual 12

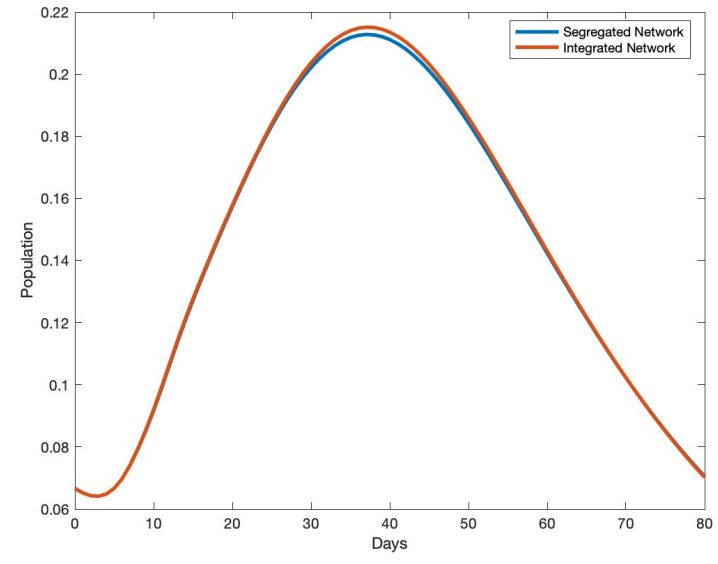

(a) Dynamic of Infection

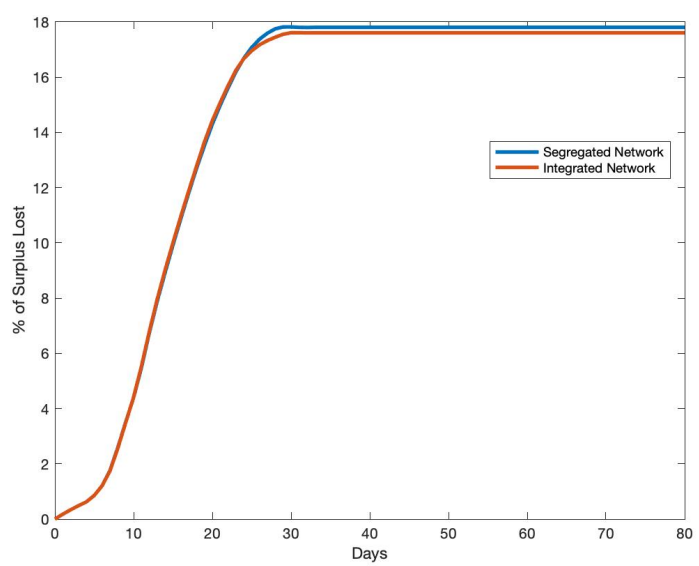

(c) Dynamic of Economic Cost

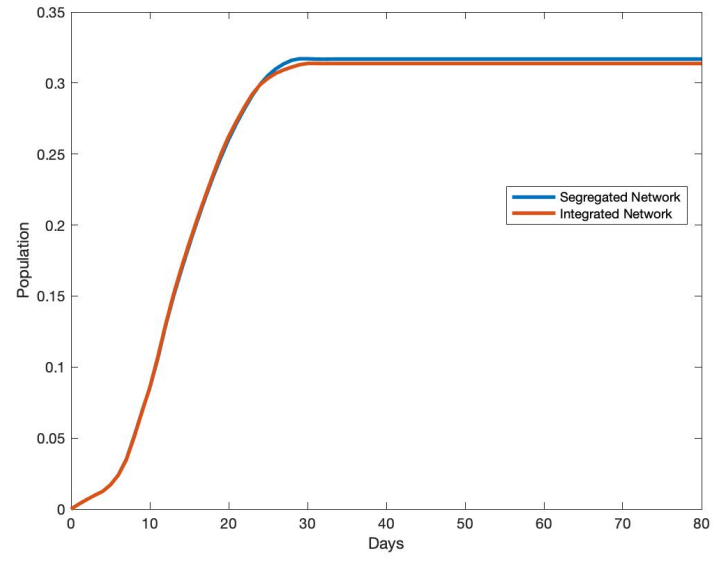

(b) Dynamic of Lockdown

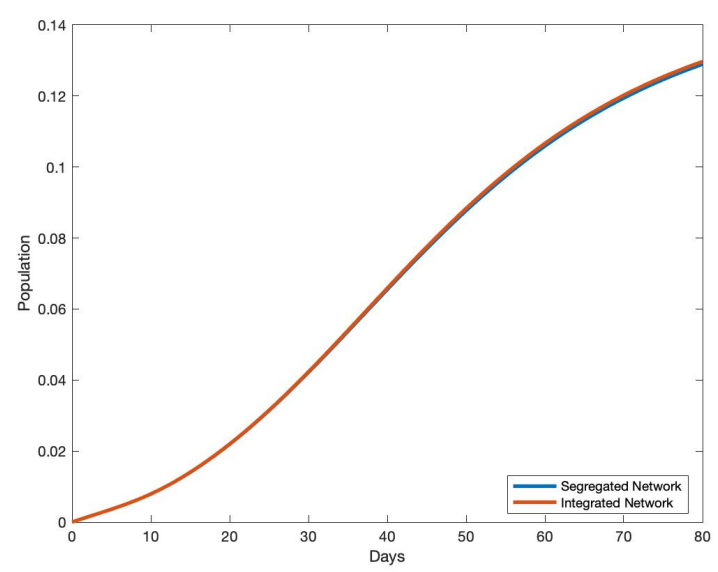

(d) Dynamic of Death 
Figure 55: Dynamics of Outcomes under the Optimal Lockdown Policy: Minority Group (1 to 5) Patient Zero: Individual 12

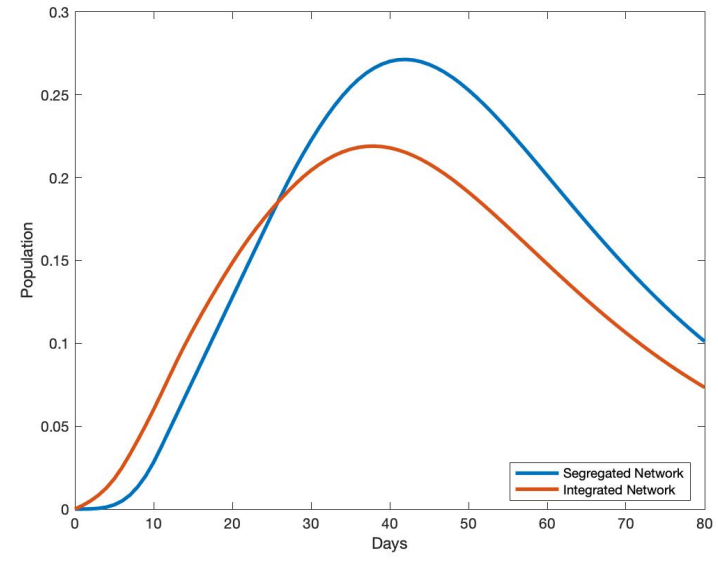

(a) Dynamic of Infection

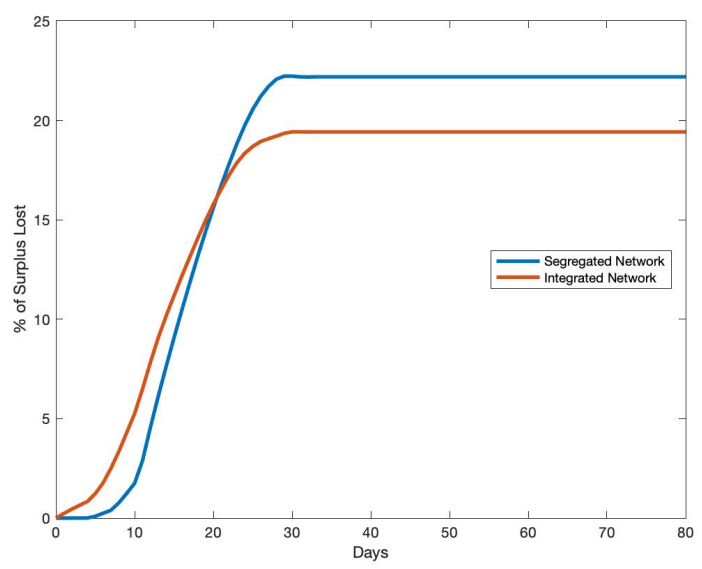

(c) Dynamic of Economic Cost

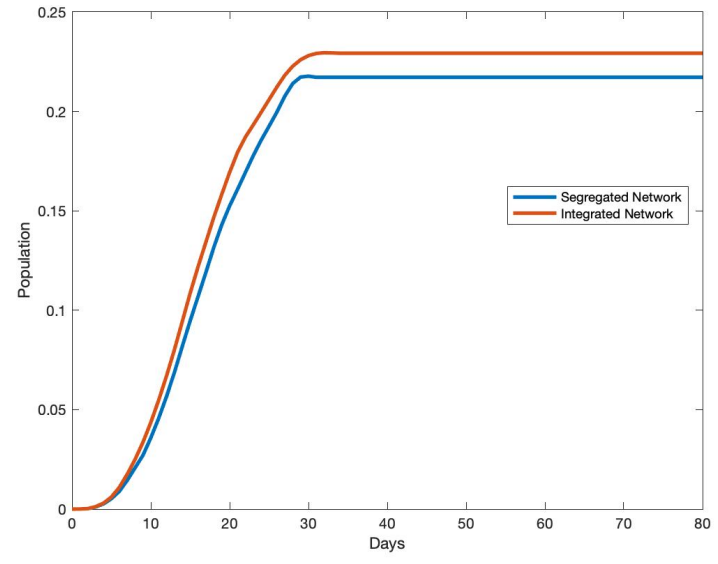

(b) Dynamic of Lockdown

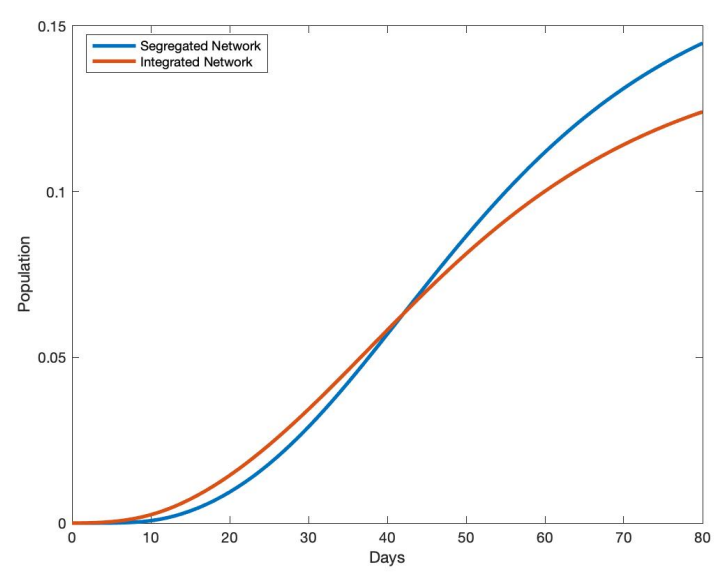

(d) Dynamic of Death 
Figure 56: Dynamics of Outcomes under the Optimal Lockdown Policy: Majority Group (6 to 15)

Patient Zero: Individual 12

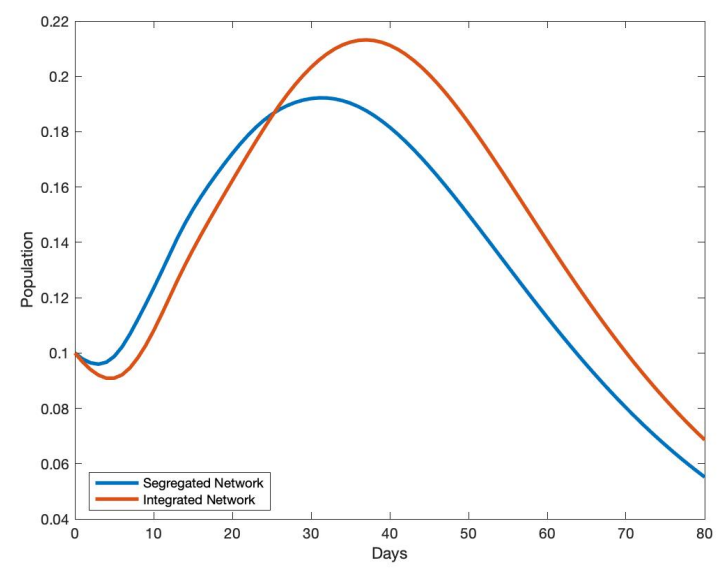

(a) Dynamic of Infection

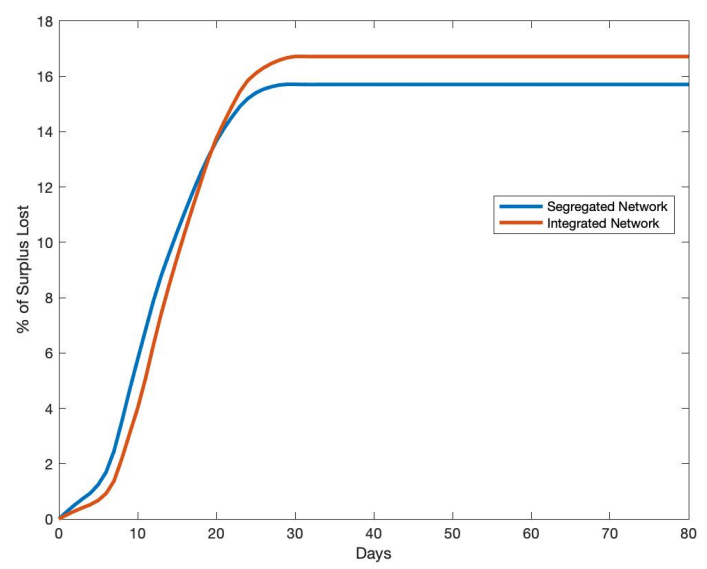

(c) Dynamic of Economic Cost

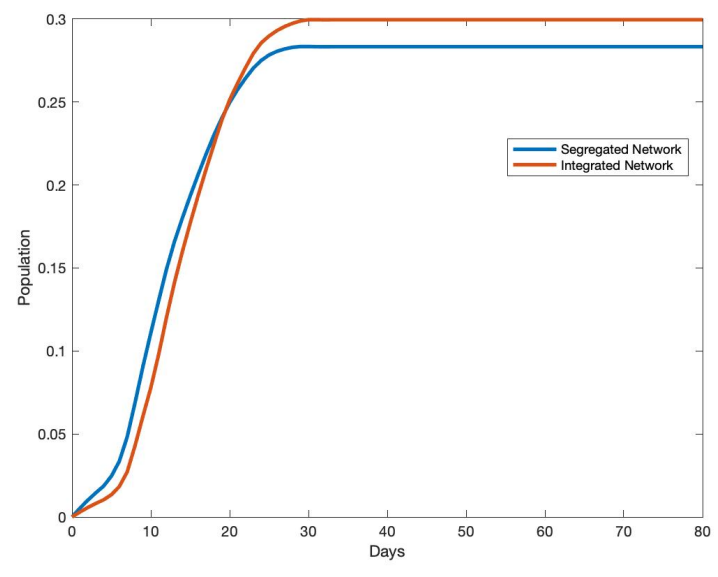

(b) Dynamic of Lockdown

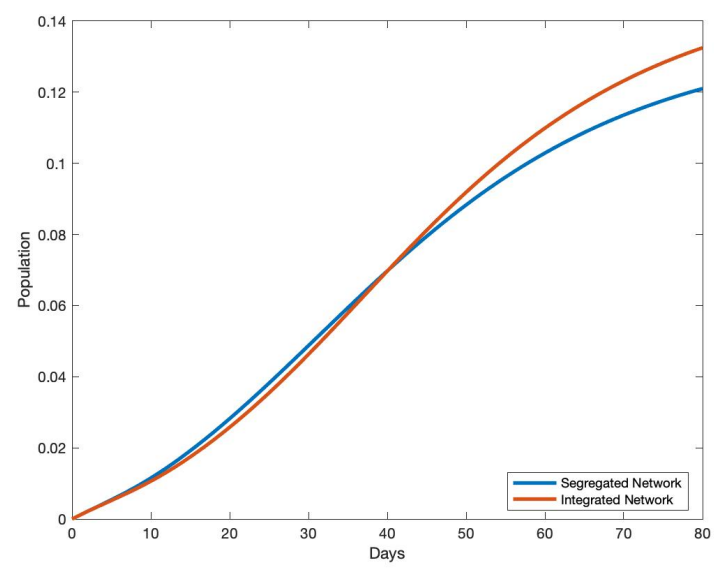

(d) Dynamic of Death 
Figure 57: Effect of Segregation on Minority-Majority Differences in Outcomes: Diff-in-Diffs Analysis

Patient Zero: Individual 12

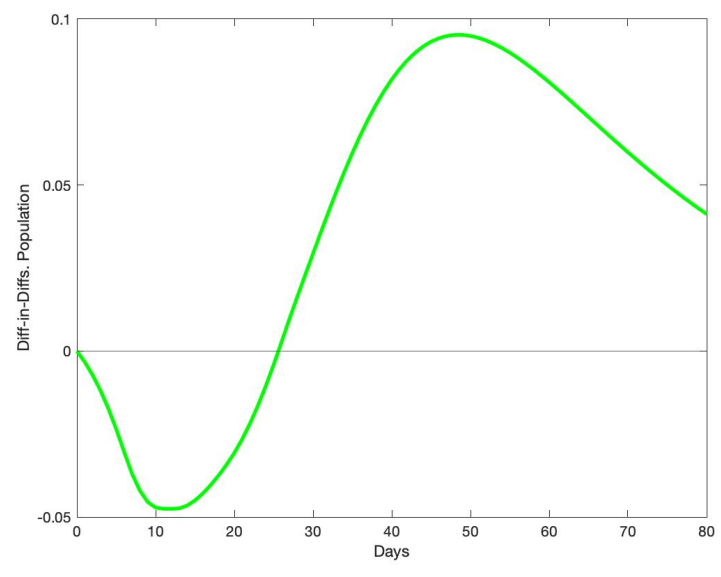

(a) Infection

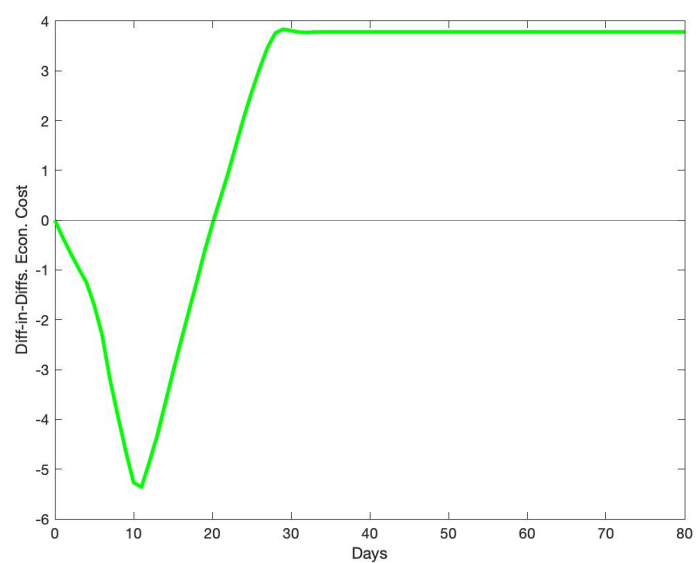

(c) Economic Cost

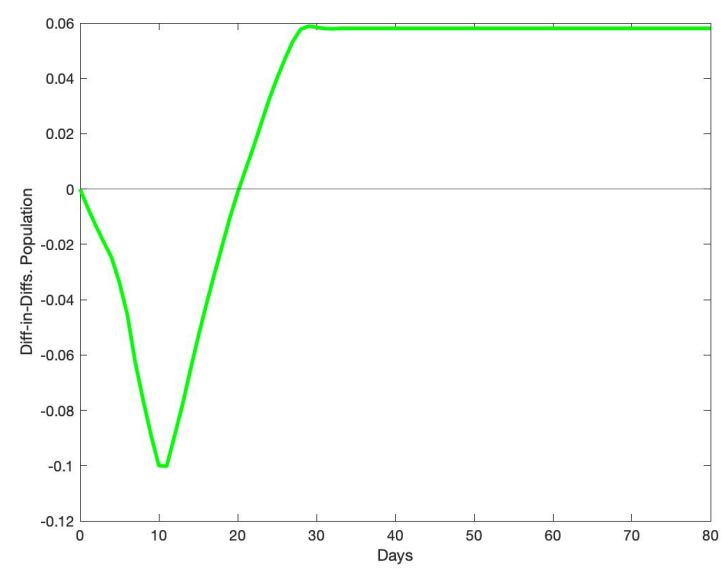

(b) Lockdown

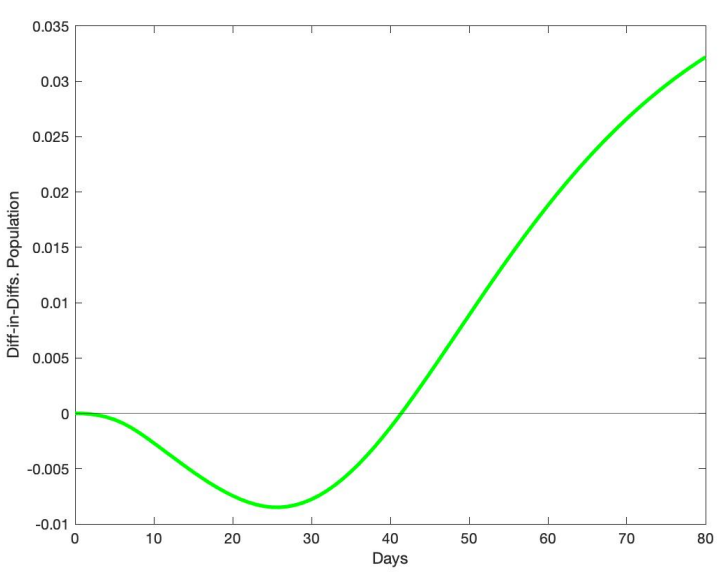

(d) Death 
Figure 58: Dynamics of Outcomes under the Optimal Lockdown Policy: Whole Population Patient Zero: Individual 13

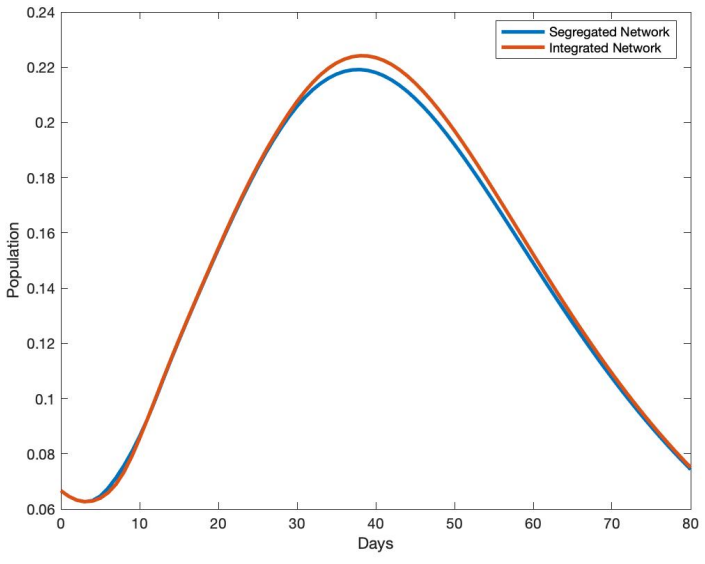

(a) Dynamic of Infection

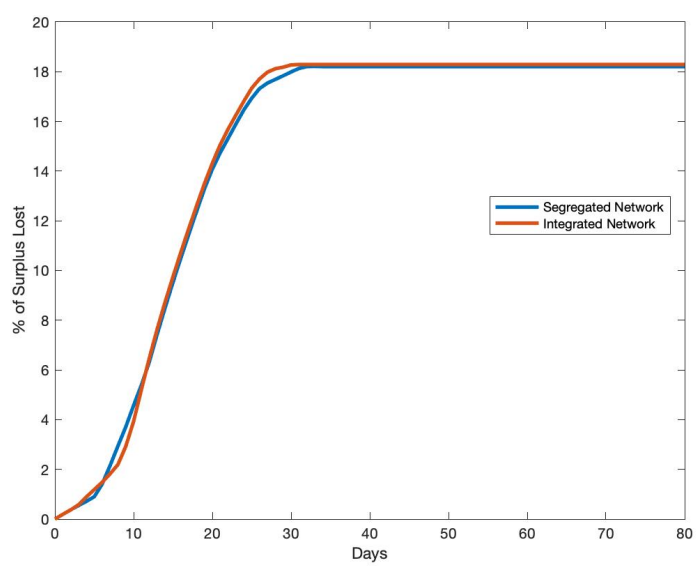

(c) Dynamic of Economic Cost

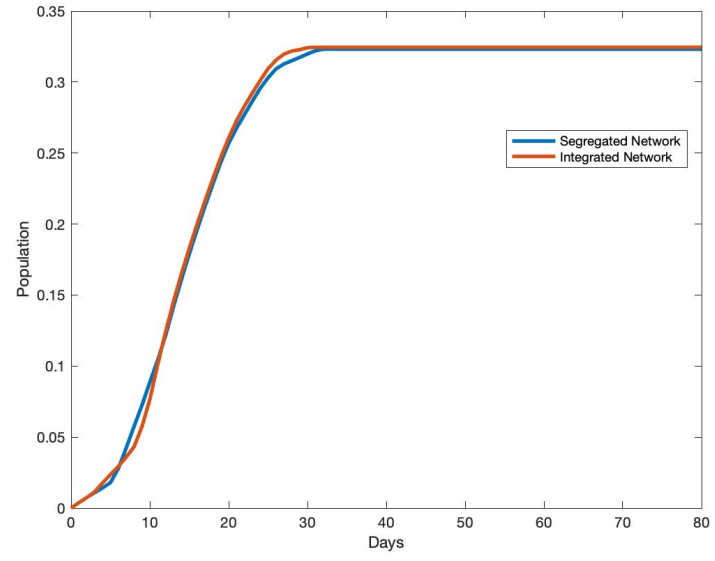

(b) Dynamic of Lockdown

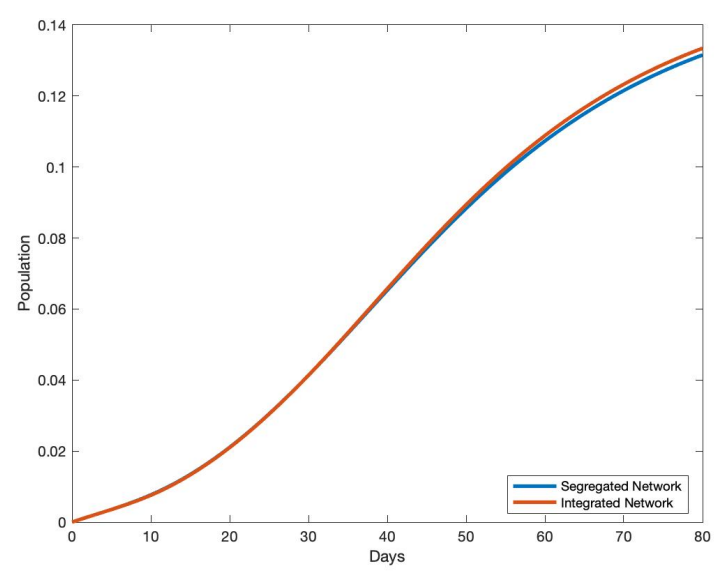

(d) Dynamic of Death 
Figure 59: Dynamics of Outcomes under the Optimal Lockdown Policy: Minority Group (1 to 5) Patient Zero: Individual 13

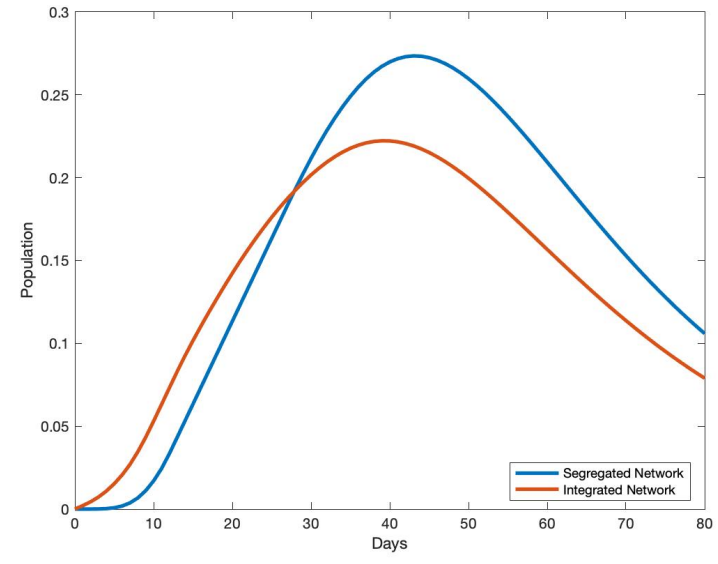

(a) Dynamic of Infection

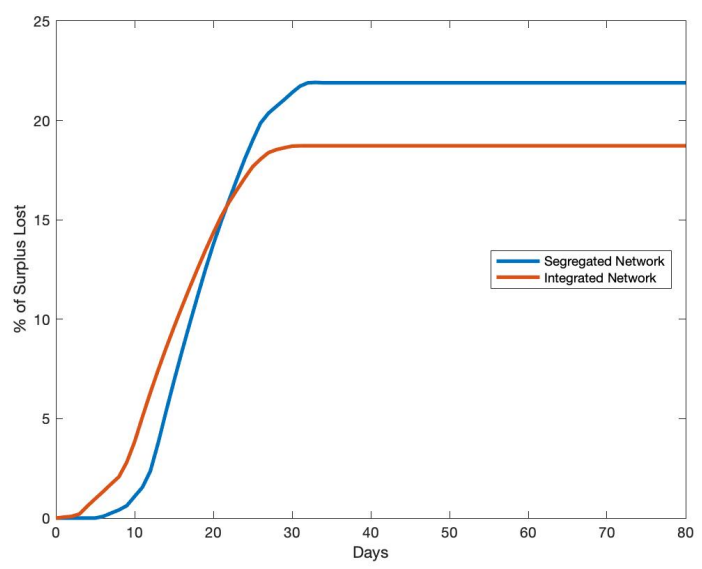

(c) Dynamic of Economic Cost

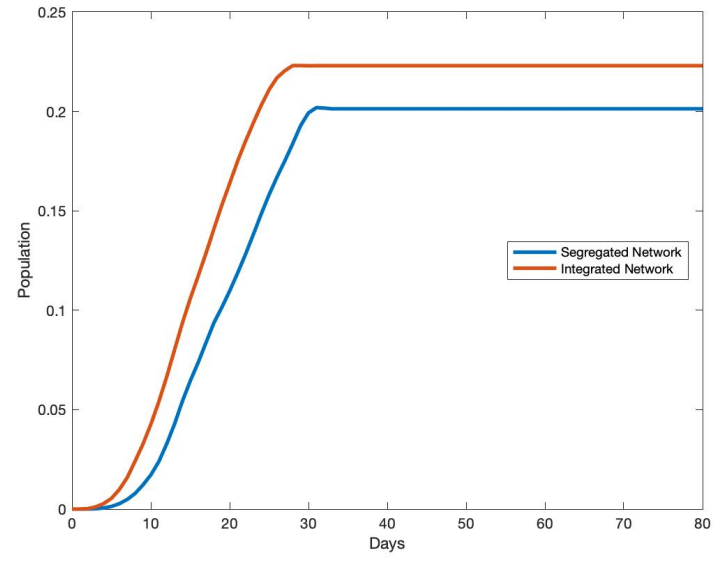

(b) Dynamic of Lockdown

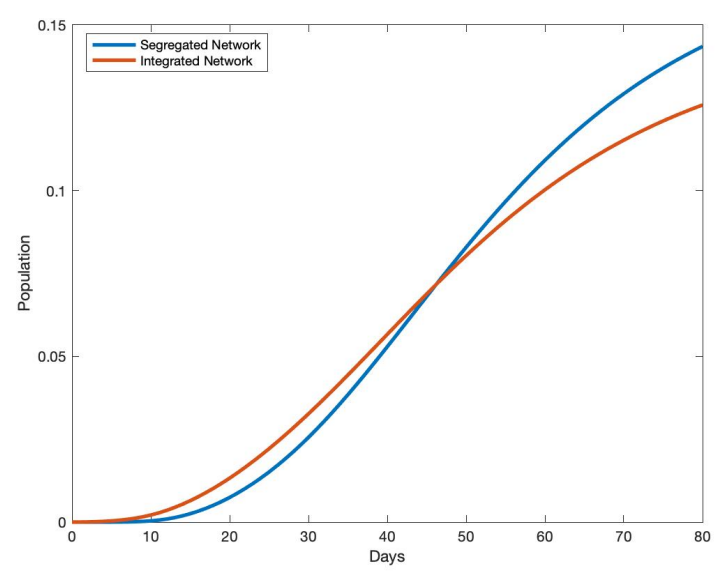

(d) Dynamic of Death 
Figure 60: Dynamics of Outcomes under the Optimal Lockdown Policy: Majority Group (6 to 15)

Patient Zero: Individual 13

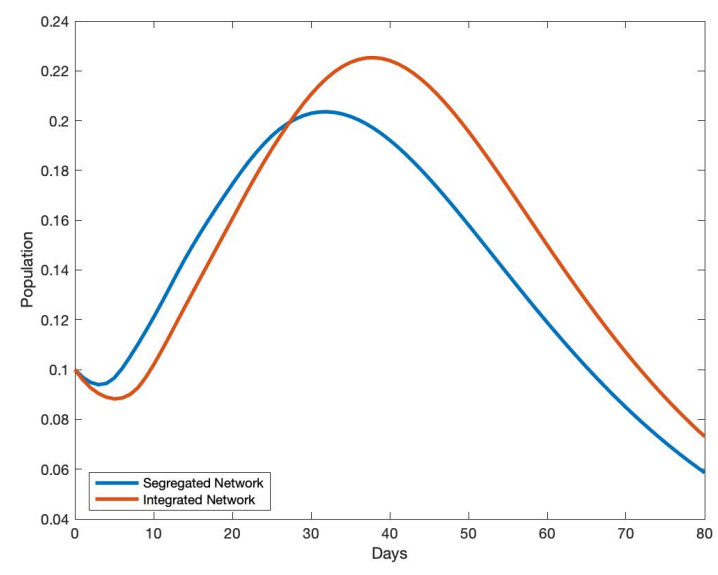

(a) Dynamic of Infection

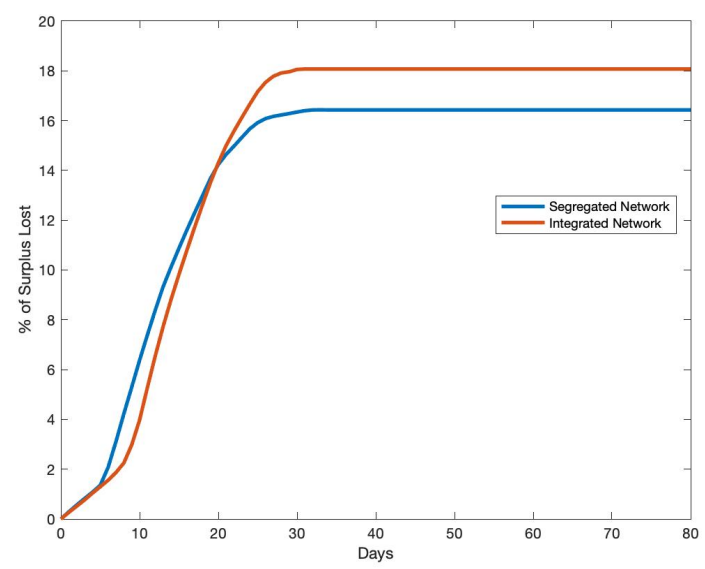

(c) Dynamic of Economic Cost

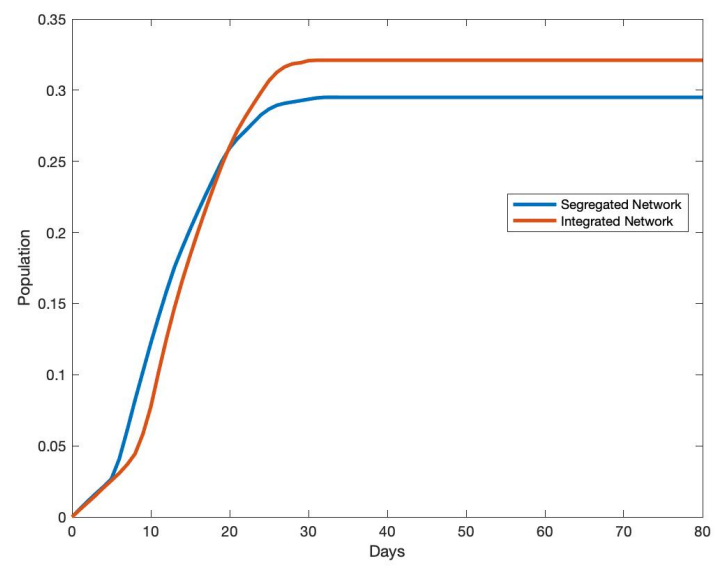

(b) Dynamic of Lockdown

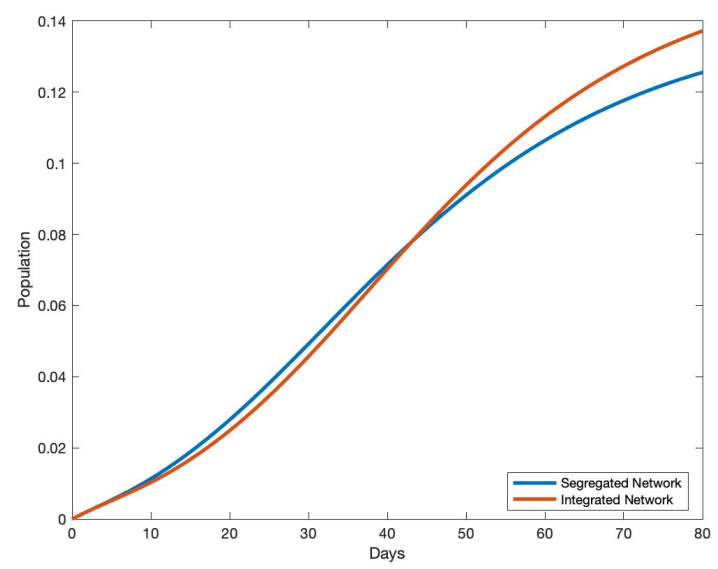

(d) Dynamic of Death 
Figure 61: Effect of Segregation on Minority-Majority Differences in Outcomes: Diff-in-Diffs Analysis

Patient Zero: Individual 13

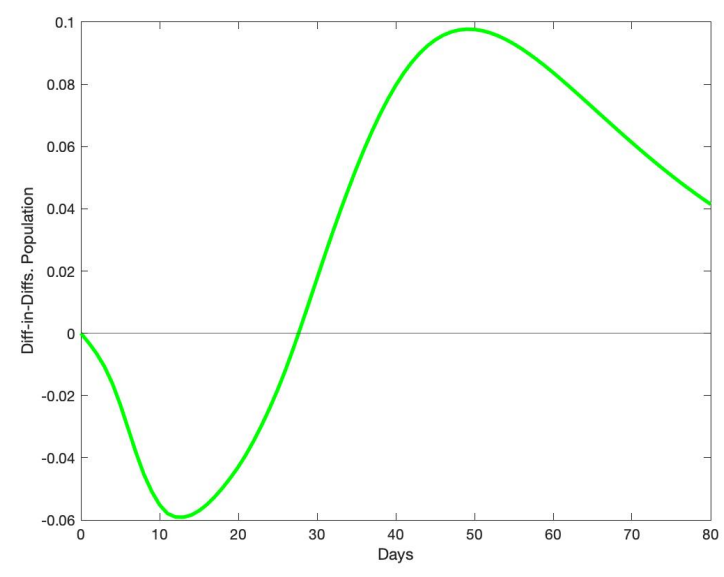

(a) Infection

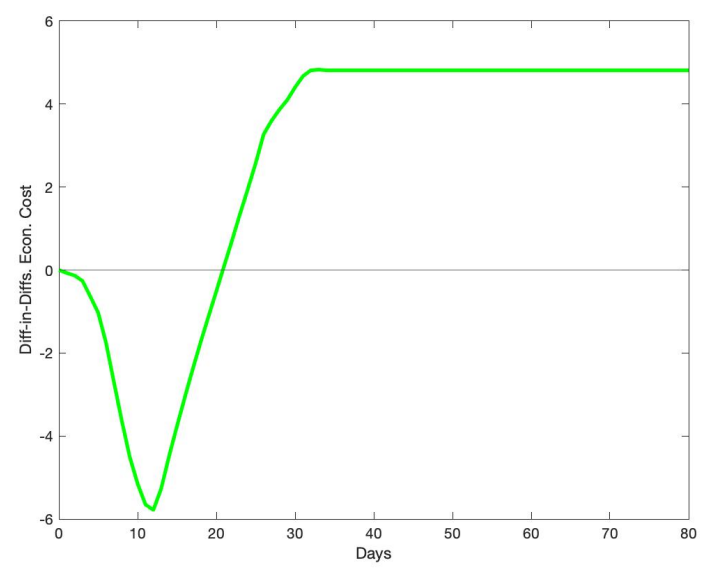

(c) Economic Cost

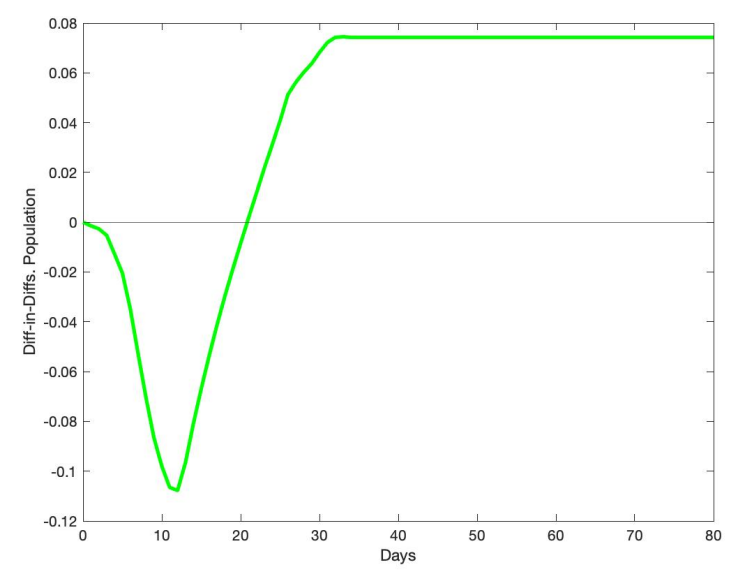

(b) Lockdown

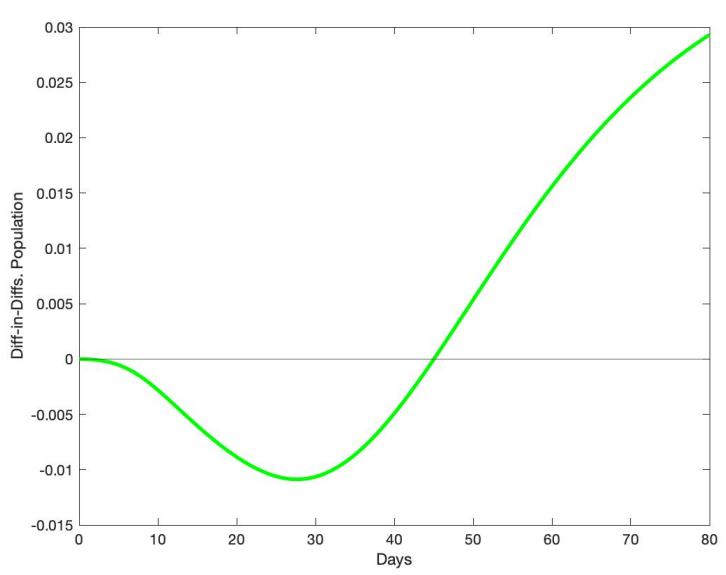

(d) Death 
Figure 62: Dynamics of Outcomes under the Optimal Lockdown Policy: Whole Population Patient Zero: Individual 14

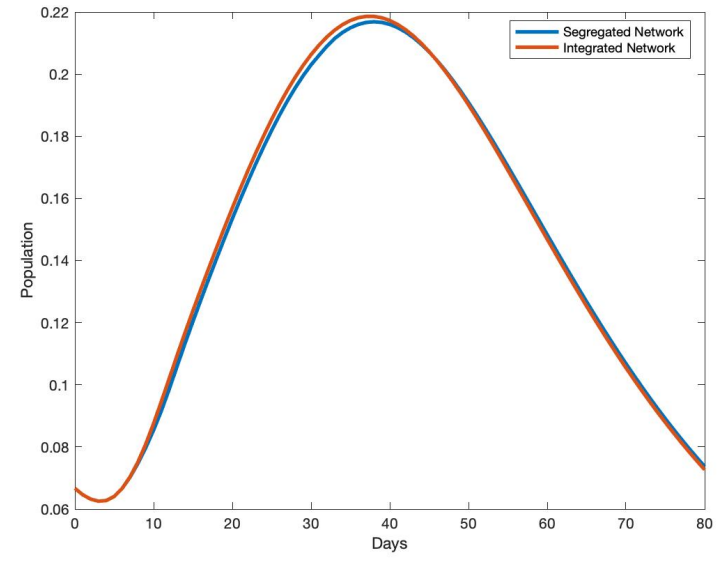

(a) Dynamic of Infection

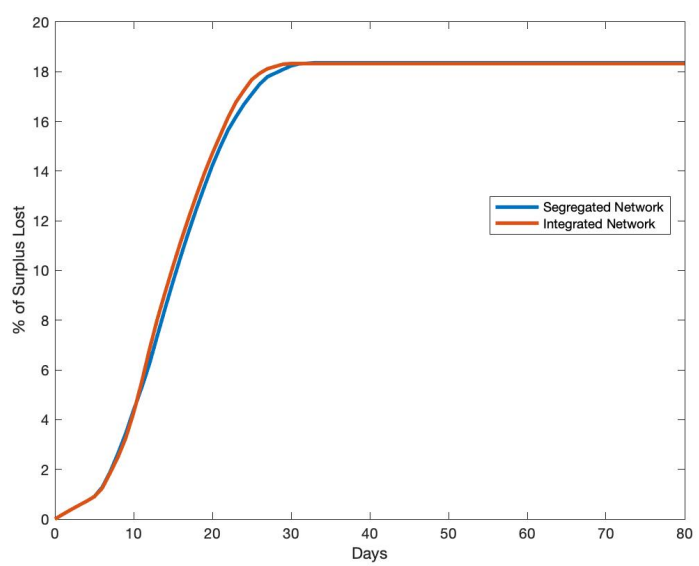

(c) Dynamic of Economic Cost

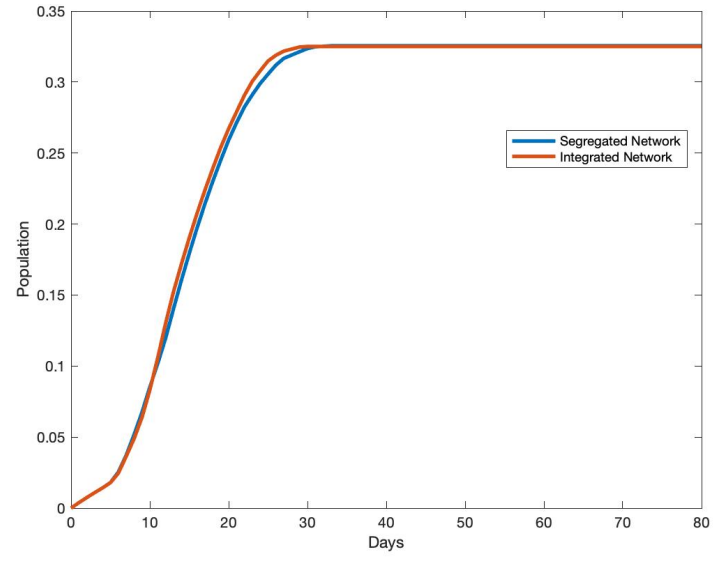

(b) Dynamic of Lockdown

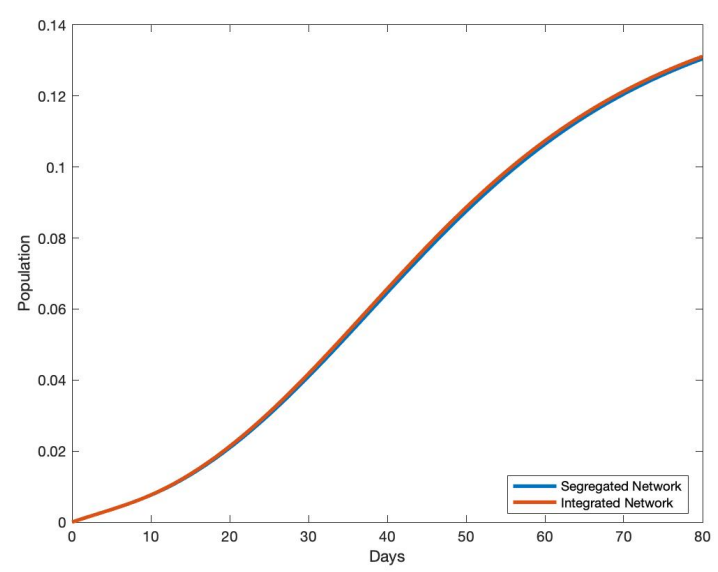

(d) Dynamic of Death 
Figure 63: Dynamics of Outcomes under the Optimal Lockdown Policy: Minority Group (1 to 5) Patient Zero: Individual 14

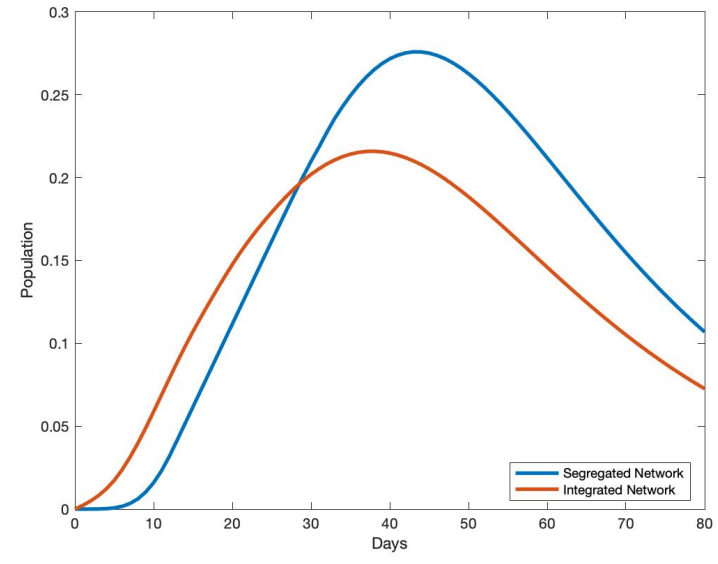

(a) Dynamic of Infection

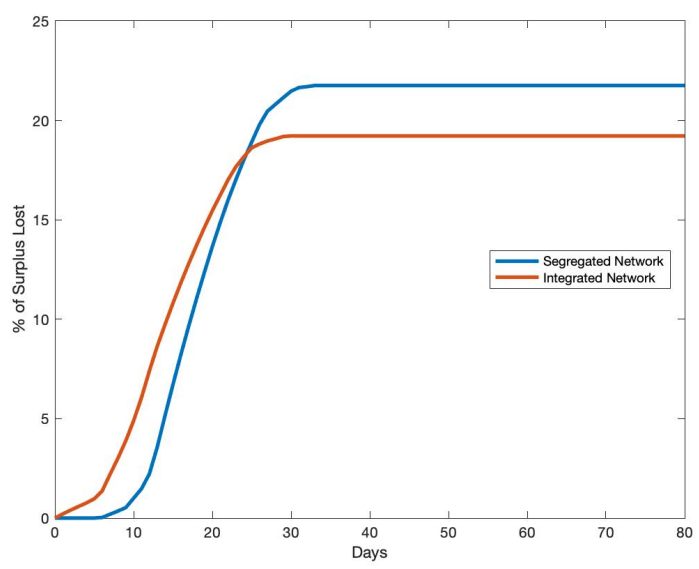

(c) Dynamic of Economic Cost

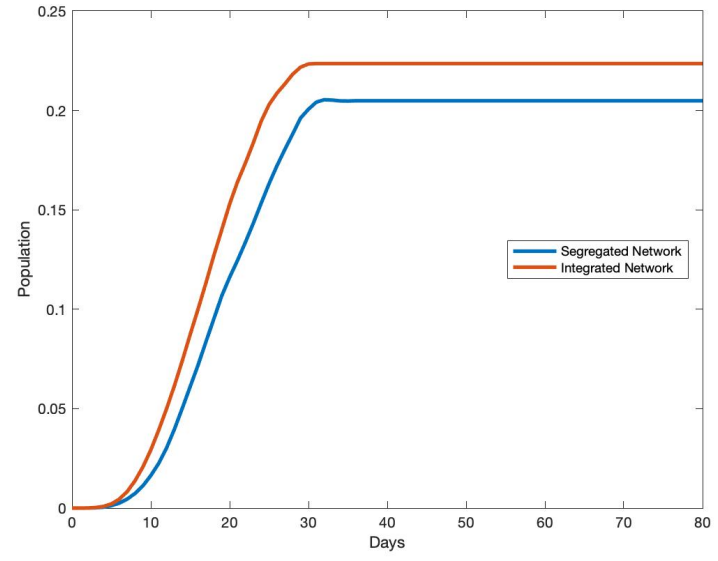

(b) Dynamic of Lockdown

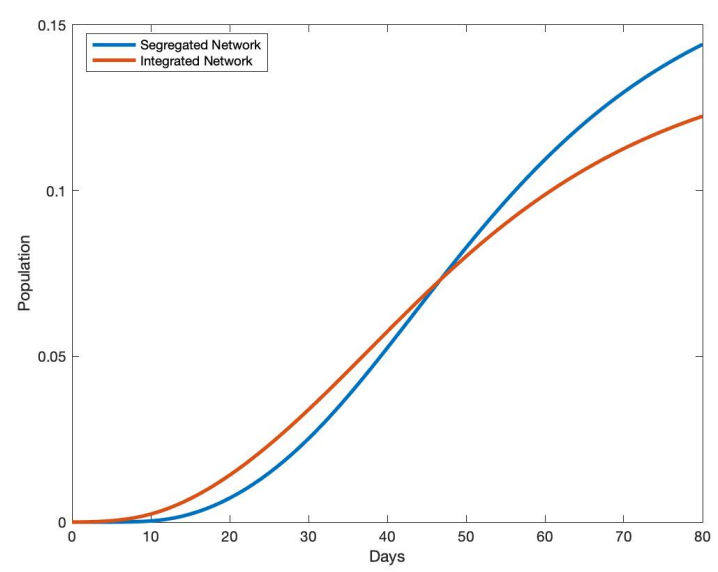

(d) Dynamic of Death 
Figure 64: Dynamics of Outcomes under the Optimal Lockdown Policy: Majority Group (6 to 15)

Patient Zero: Individual 14

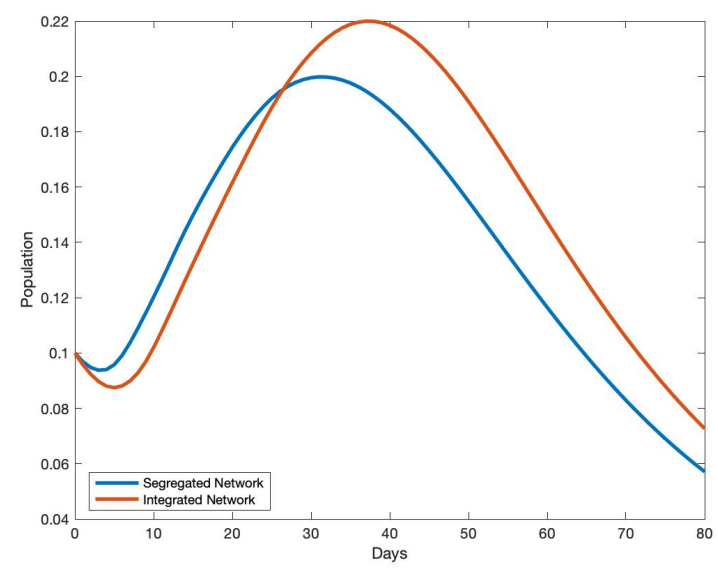

(a) Dynamic of Infection

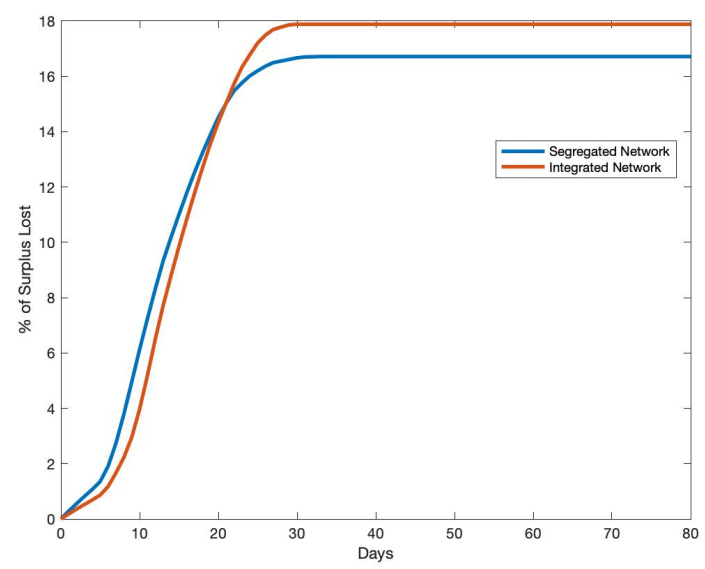

(c) Dynamic of Economic Cost

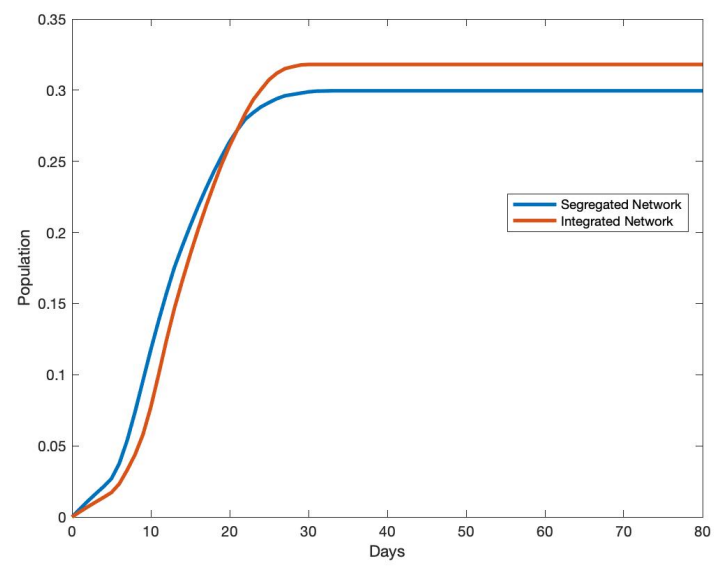

(b) Dynamic of Lockdown

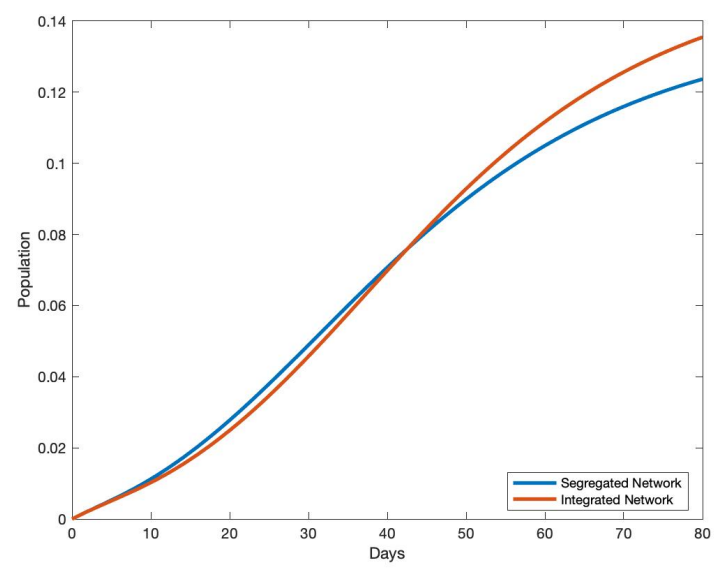

(d) Dynamic of Death 
Figure 65: Effect of Segregation on Minority-Majority Differences in Outcomes: Diff-in-Diffs Analysis

Patient Zero: Individual 14

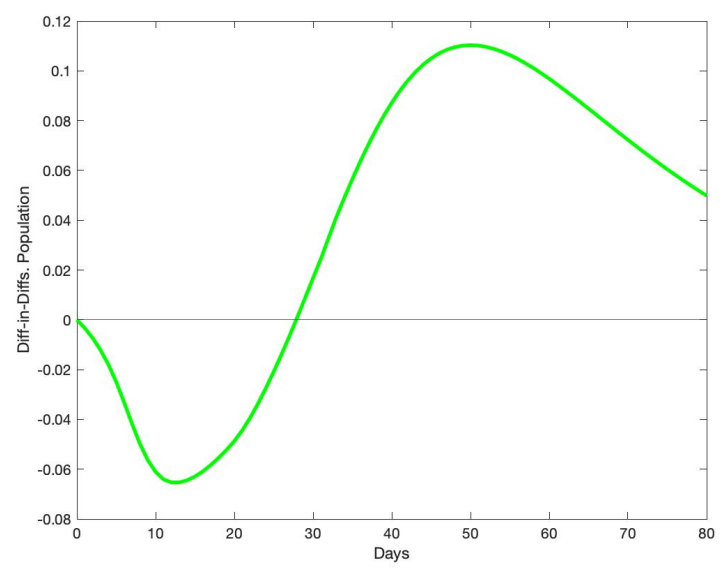

(a) Infection

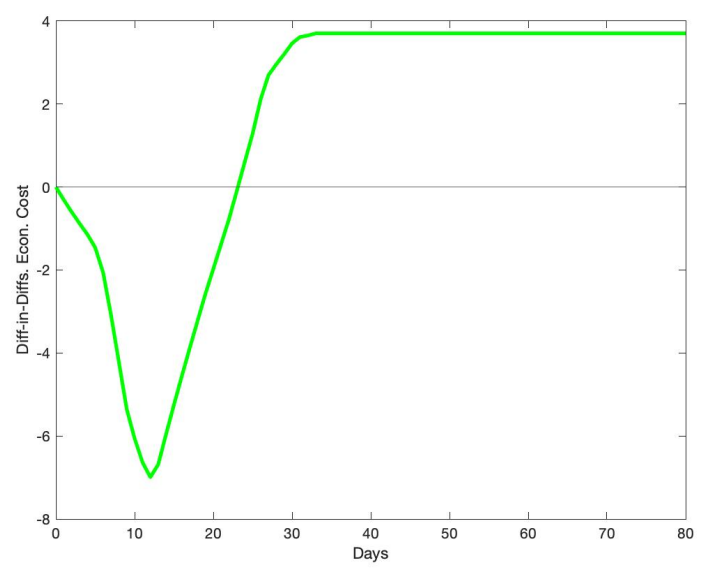

(c) Economic Cost

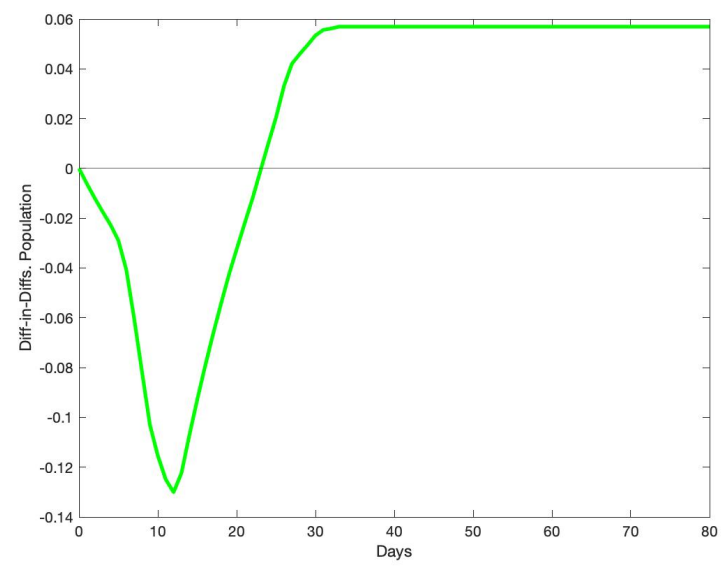

(b) Lockdown

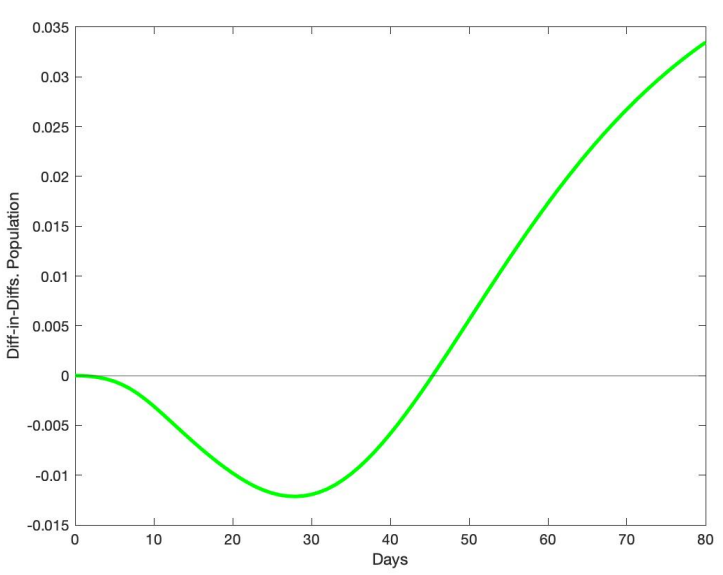

(d) Death 
Figure 66: Dynamics of Outcomes under the Optimal Lockdown Policy: Whole Population Patient Zero: Individual 15

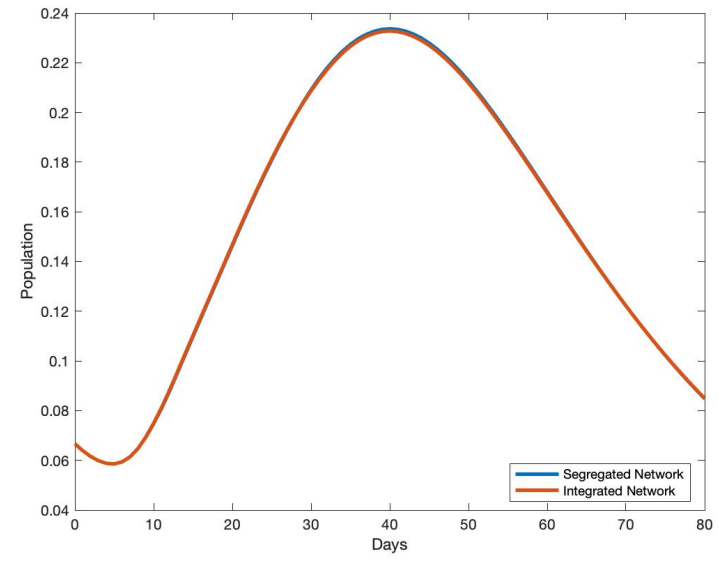

(a) Dynamic of Infection

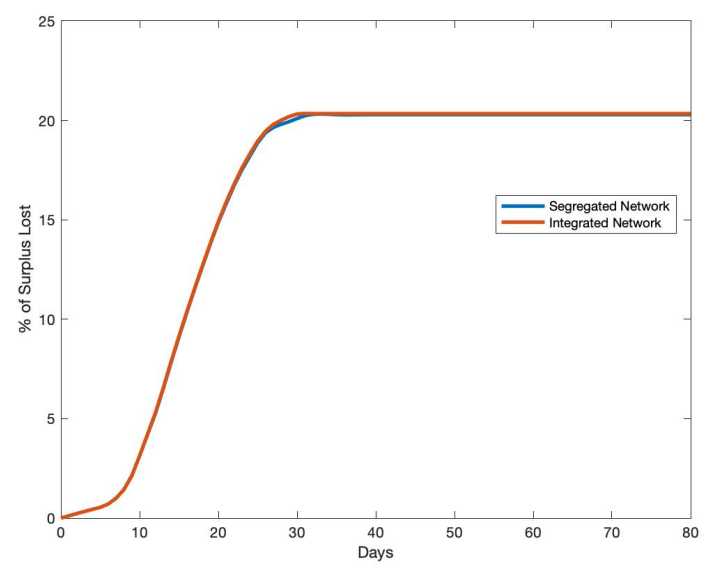

(c) Dynamic of Economic Cost

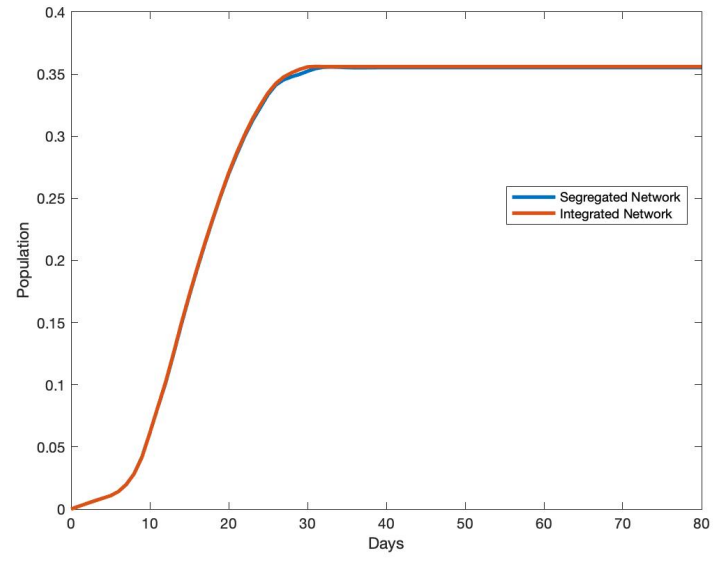

(b) Dynamic of Lockdown

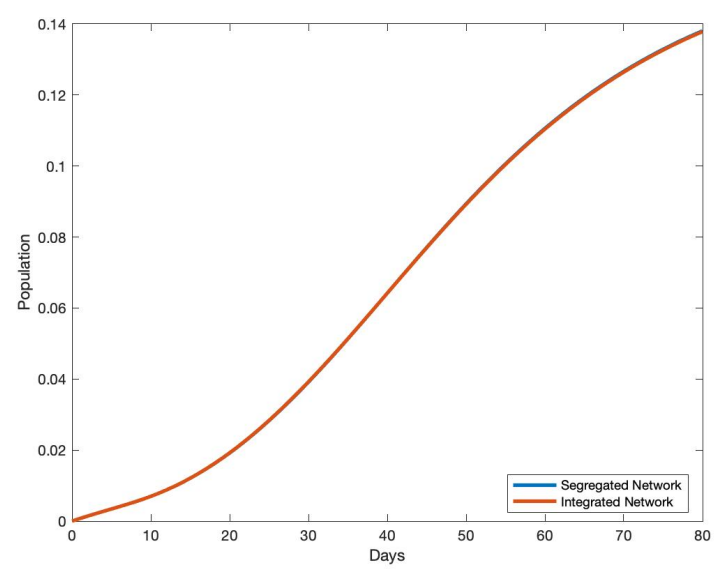

(d) Dynamic of Death 
Figure 67: Dynamics of Outcomes under the Optimal Lockdown Policy: Minority Group (1 to 5) Patient Zero: Individual 15

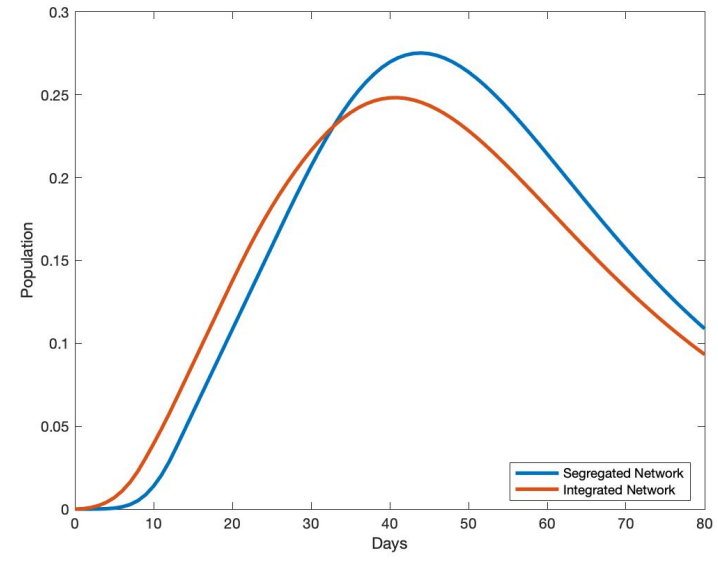

(a) Dynamic of Infection

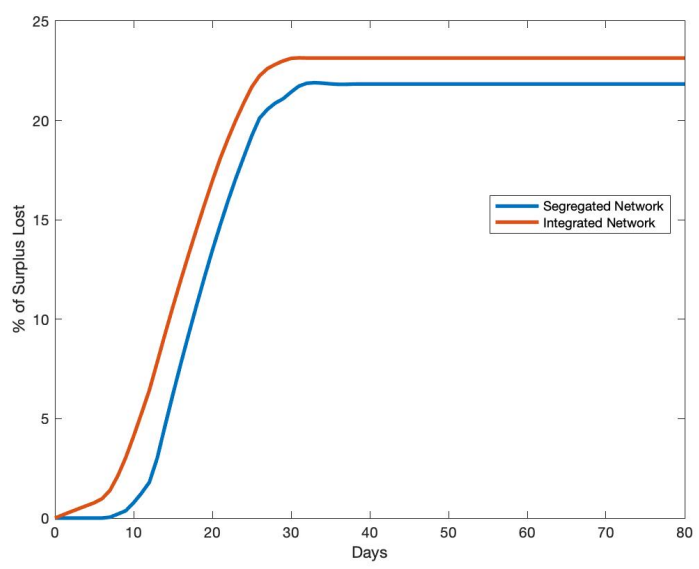

(c) Dynamic of Economic Cost

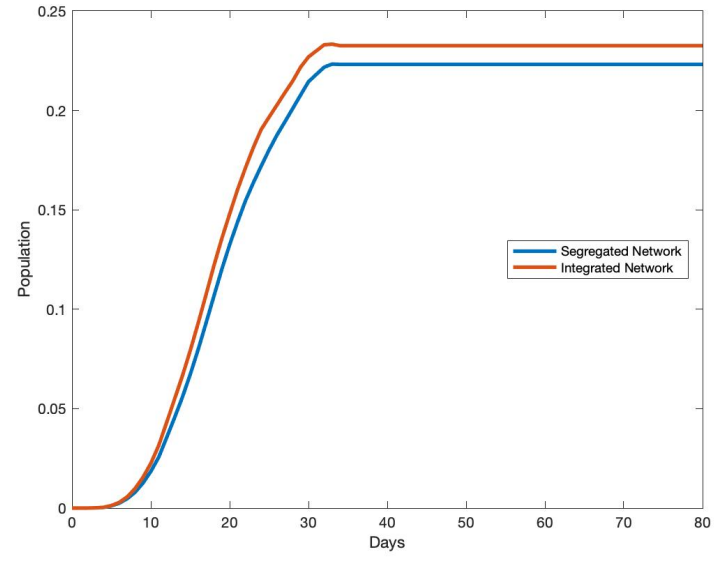

(b) Dynamic of Lockdown

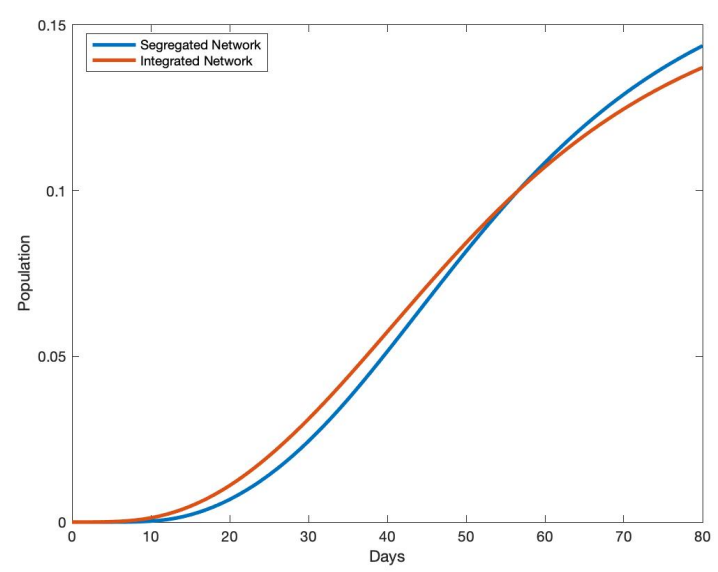

(d) Dynamic of Death 
Figure 68: Dynamics of Outcomes under the Optimal Lockdown Policy: Majority Group (6 to 15) Patient Zero: Individual 15

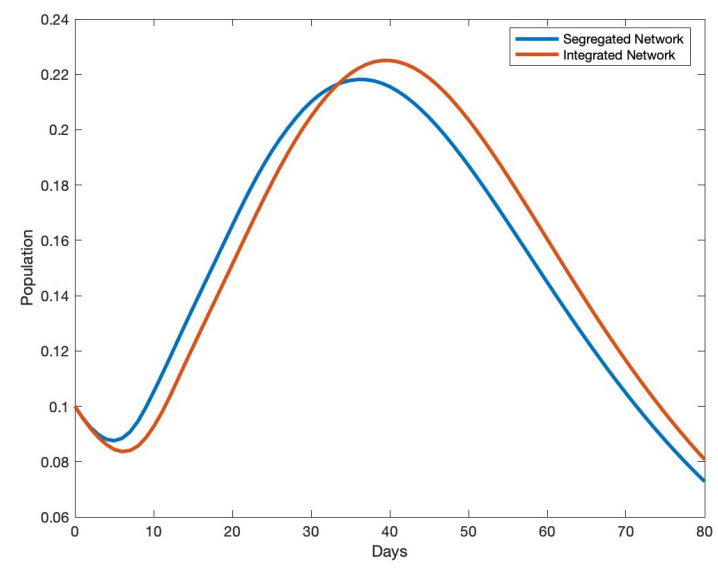

(a) Dynamic of Infection

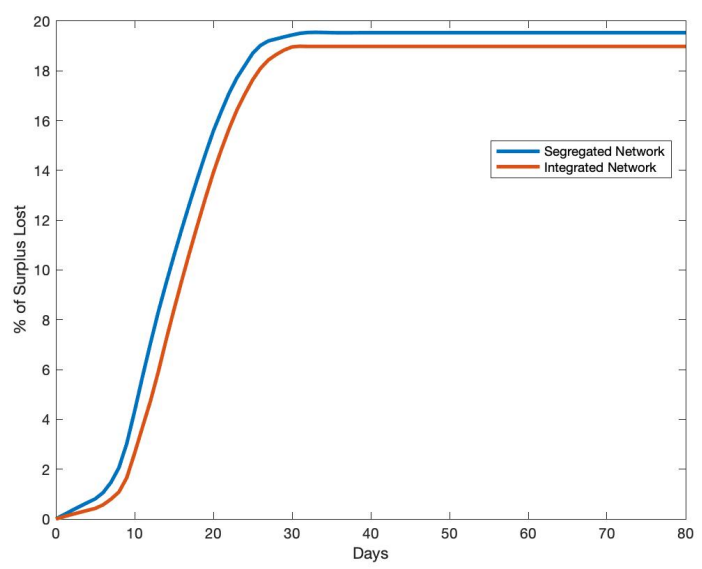

(c) Dynamic of Economic Cost

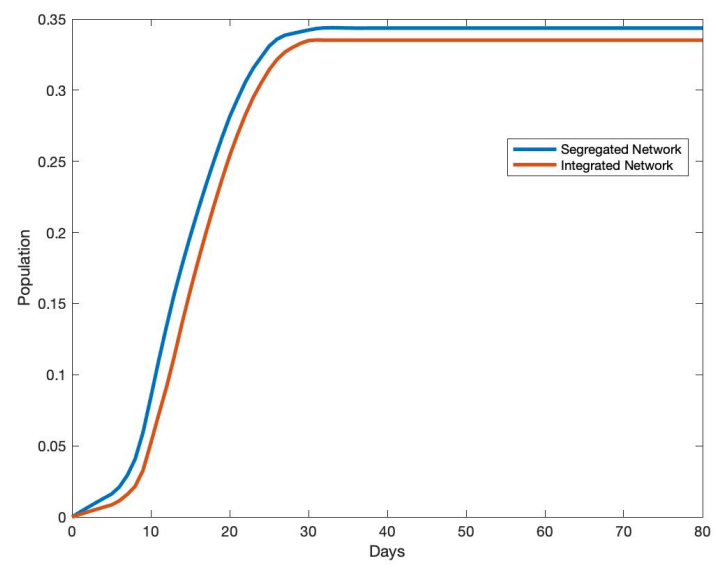

(b) Dynamic of Lockdown

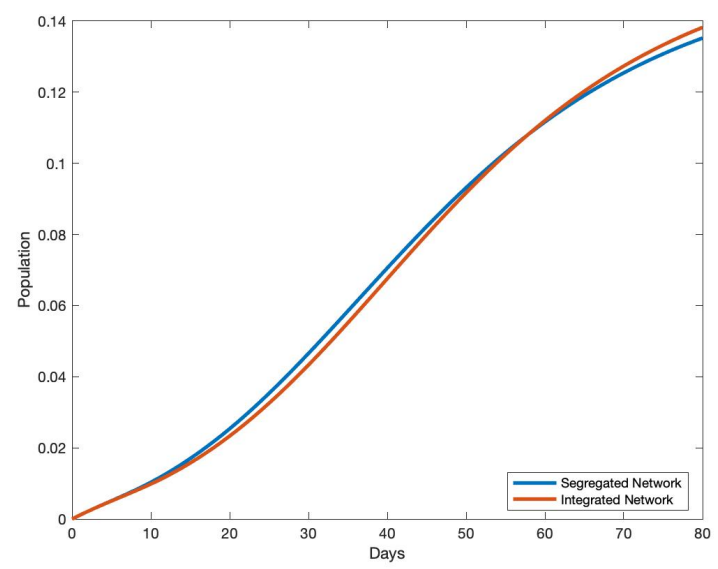

(d) Dynamic of Death 
Figure 69: Effect of Segregation on Minority-Majority Differences in Outcomes: Diff-in-Diffs Analysis

Patient Zero: Individual 15

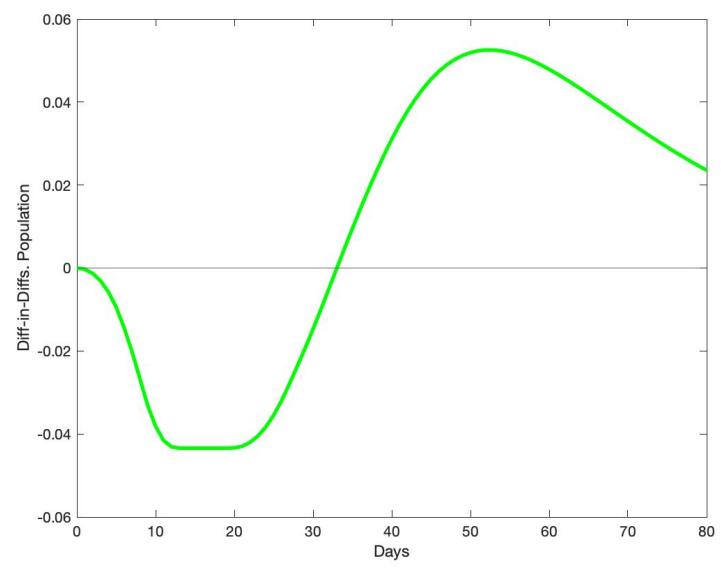

(a) Infection

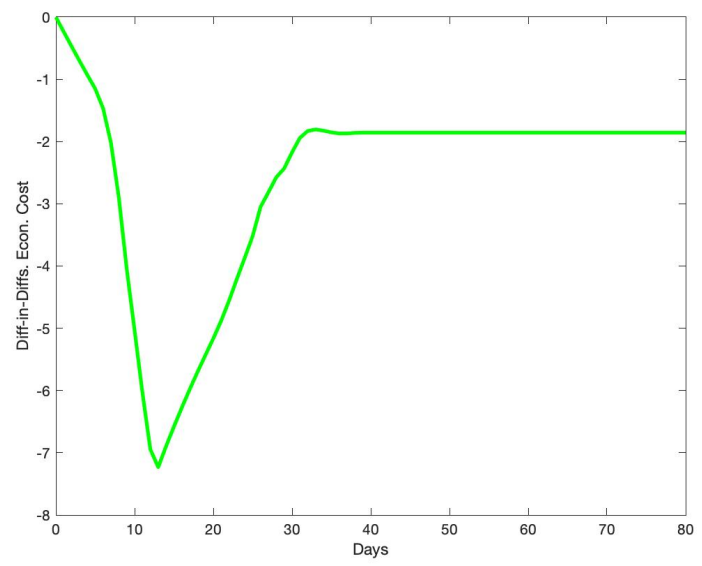

(c) Economic Cost

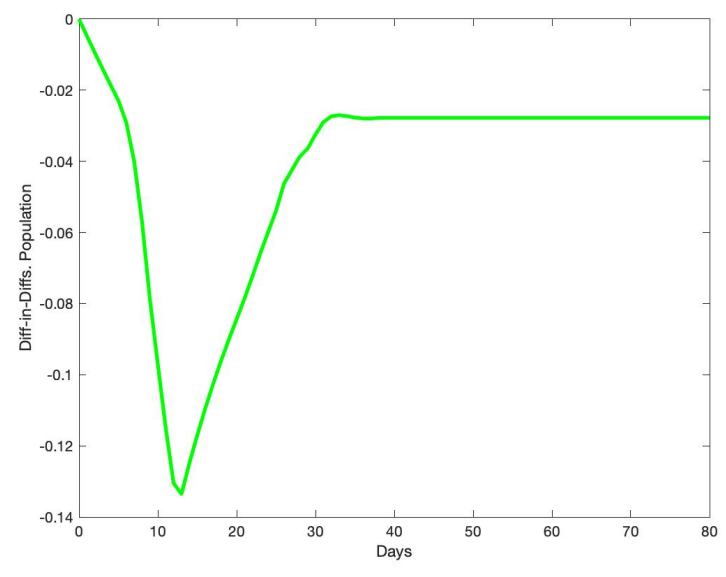

(b) Lockdown

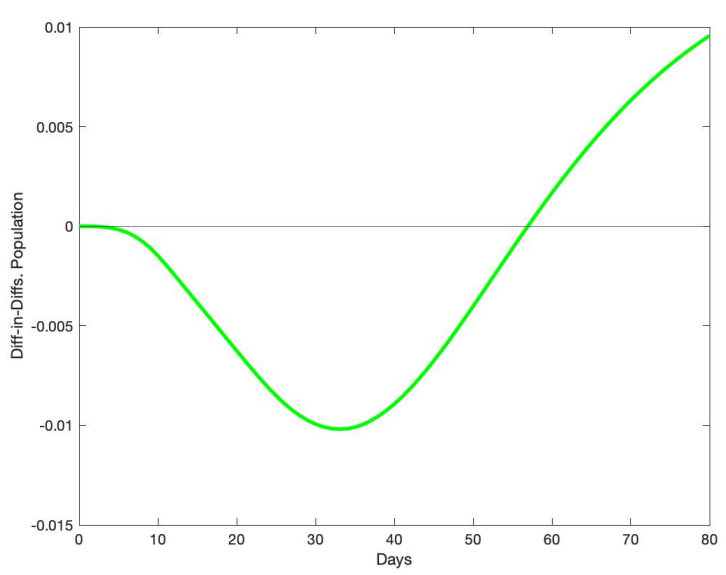

(d) Death 